Supporting Information

\title{
Chiral Electron-Rich PNP Ligand with a Phospholane Motif: Structural Feature and Application in Asymmetric Hydrogenation
}

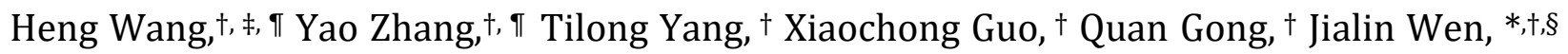
and Xumu Zhang*,†

†Shenzhen Grubbs Institute and Department of Chemistry, Southern University of Science and Technology, Shenzhen 518055, China

‡ Department of Chemistry, Temple University, Philadelphia, PA, 19122, United States.

$\S$ Academy for Advanced Interdisciplinary Studies, Southern University of Science and Technology, Shenzhen 518055, China

\section{Contents}

1. General remarks. S2

2. Synthesis of ligand and complexes. . $\mathrm{S} 3$

3. Synthesis of biaryl ketones. S8

4. General procedure for iridium catalyzed hydrogenation. S14

5. Characterization data of the hydrogenation products. S18

6. Computation studies. S41

7. Synthesis and reactivity of $\left[\mathrm{FeBr}_{2}(\mathrm{Heng}-\mathrm{PNP})\right]$ (6) and structural determination. S41

8. NMR spectra of all the compounds and complexes. S51

References. S109 


\section{General remarks.}

All the reactions dealing with air- or moisture- sensitive compounds were carried out with standard Schlenk technique in a reaction vessel which was dried by a heating gun or carried out in an argon-filled glove box. Unless otherwise noted, $((1 R, 2 R)$-cyclohexane-1,2-diyl)dimethanol and all the other reagents were purchased from commercial suppliers without further purification. Solvents for complex and ligand synthesis such as THF, toluene, hexane was dried with sodium chips and distilled over benzophenone, after which the solvent were further degassed by freeze-pump-thaw technique. Anhydrous dichloromethane and ethanol were purchased from J\&K Chemical and degassed with freeze-pump-thaw. Deionized water was degassed by freeze-pump-thaw. Solvents for reaction condition screening were purchased from J\&K Chemical and degassed though bubbling argon for 2 hours. Purification of products was carried out via flash chromatography on silica (200-300 mesh). Thin layer chromatography was carried out using silica plates from Merck (GF254). Zinc powder (325 mesh) and anhydrous $\mathrm{FeBr}_{2}$ was purchased from Sigma-Aldrich, $[\operatorname{Ir}(\mathrm{COD}) \mathrm{Cl}]_{2}$ was purchased from Heraeus, $[\operatorname{Ir}(\mathrm{COE}) \mathrm{Cl}]_{2}$ was prepared according to the reference. Deuterated solvents were purchased from Cambridge Isotope Laboratories and degassed through freezepump-thaw, dried with $3 \AA$ molecular sieves in a glove box.

${ }^{1} \mathrm{H}$ NMR, ${ }^{13} \mathrm{C}$ NMR, ${ }^{31} \mathrm{P}$ NMR spectra were obtained at room temperature on a Bruker AV400 MHz or Bruker AV600 MHz spectrometer with chemical shifts (d) referred to the residual solvent signal. Chemical shifts were reported upfield to TMS $(0.00 \mathrm{ppm})$ for ${ }^{1} \mathrm{HNMR}$ and relative to $\mathrm{CDCl}_{3}(77.0 \mathrm{ppm})$ for ${ }^{13} \mathrm{C}$ NMR. Data were reported as: multiplicity ( $\mathrm{s}=$ singlet, $\mathrm{d}=$ doublet, $\mathrm{t}=$ triplet, $\mathrm{q}=$ quartet, $\mathrm{m}=$ multiplet $)$, coupling constant in herz $(\mathrm{Hz})$ and signal

area integration in natural numbers. ${ }^{13} \mathrm{C}$ NMR and ${ }^{31} \mathrm{P}$ NMR spectra were recorded with ${ }^{1} \mathrm{H}$ decoupling. Enantiomeric excess values were determined by Agilent 1290 Series HPLC instrument on a chiral stationary phase. Optical rotations were measured using $1 \mathrm{~mL}$ cell with a $1 \mathrm{dm}$ path length on a Rudolph Autopol I polarimeter at $589 \mathrm{~nm}$. High resolution mass spectra (HRMS) were obtained on Thermo Scientific Q Exactive hybrid quadrupole-Orbitrap mass spectrometer. A positive ion mass spectrum of sample was acquired on a Thermo LTQ-FT mass spectrometer with an electrospray ionization source. Crystal structure was measured with BRUKER APEX III diffractometer. 


\section{Synthesis of ligand and complexes.}

Synthesis of the ligand:
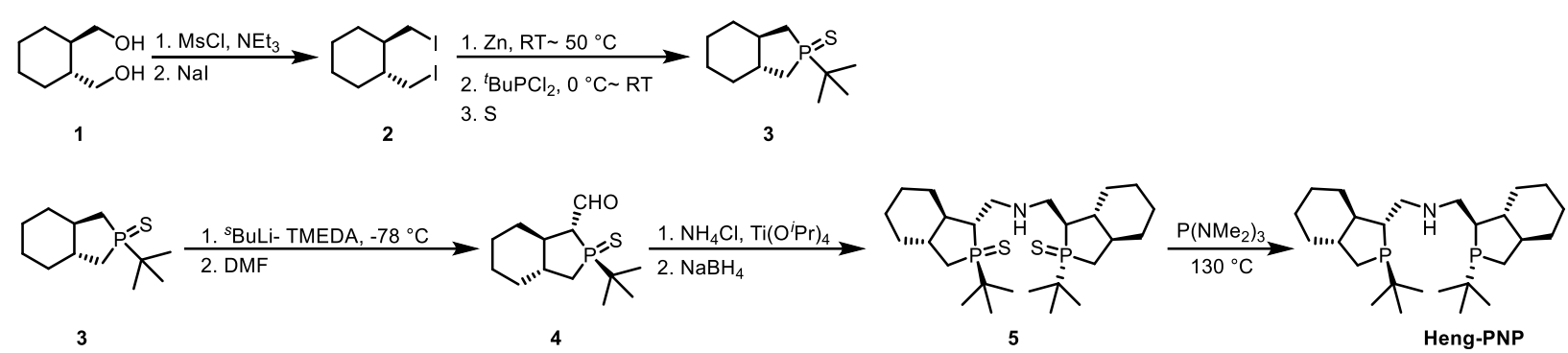

\subsection{Synthesis of $(1 R, 2 R)$-1,2-bis(iodomethyl)cyclohexane (2)}

Compound 2 was synthesized with a modified procedure ${ }^{1}$. To a 1-L heating-gun-dried three-necked flask was charged with $40 \mathrm{~g}$ of $((1 R, 2 R)$-cyclohexane-1,2-diyl)dimethanol $1(277 \mathrm{mmol}), 550 \mathrm{~mL}$ of anhydrous dichloromethane and $43 \mathrm{ml}$ of mesyl chloride (555 mmol, 2 equiv.). The flask was cooled to $0{ }^{\circ} \mathrm{C}$ and triethylamine (77 mL, $555 \mathrm{mmol}, 2$ equiv.) was added slowly. The reaction was warmed to room temperature after addition of triethylamine gradually and stirring overnight, the reaction was quenched by $300 \mathrm{~mL}$ water and washed by $\mathrm{NH}_{4} \mathrm{Cl}$ (aq.), saturated $\mathrm{NaHCO}_{3}$ (aq.) and saturated $\mathrm{NaCl}$ (aq.) successively. The organic phase was dried on anhydrous $\mathrm{NaSO}_{4}$, then solvent was removed in vacuo. The off-white residue was dried under vacuum and used directly for the next step.

A 1-L heating-gun-dried three-necked flask was equipped with a stirring bar and a condenser, $104 \mathrm{~g}$ of NaI (693 mmol, 2.5 equiv.) and the crude product in the last step was added into the reaction system, argon was refilled via Schlenk technique, then $600 \mathrm{~mL}$ of acetone was added, the reaction was covered with aluminum foil and heated in a $55{ }^{\circ} \mathrm{C}$ oil bath overnight. Solvent was removed by rotary evaporator and the residue was dried under vacuum. Then, the residue passed through a silica gel plug by hexane and afforded white crystalline product ( $80 \mathrm{~g}, 79 \%)$. The NMR spectral data matched with values in literature.

\subsection{Synthesis of (3aR,7aR)-2-(tert-butyl)octahydroisophosphindole 2-sulfide (3)}

Compound $\mathbf{3}$ was synthesized with a modified procedure ${ }^{2}$. In a 100-mL heating-gun-dried three-necked flask zinc powder (2.6 g, $40 \mathrm{mmol}, 4$ equiv) was charged. Then anhydrous THF ( $3 \mathrm{~mL}$ ) and 1,2-dibromoethane ( $0.2 \mathrm{~mL}, 2 \mathrm{mmol})$ were added. The flask was heated with a heating gun until reflux for $1 \mathrm{~min}$ and then cooled to room temperature. This operation was repeated for 8-10 times and then chlorotrimethylsilane $(0.26 \mathrm{~mL}, 2 \mathrm{mmol})$ was added. The resulting mixture was stirred for additional $30 \mathrm{~min}$. Compound $2(3.64 \mathrm{~g}, 10 \mathrm{mmol})$ in $20 \mathrm{~mL}$ THF $(0.5 \mathrm{M})$ was added into the 
reaction system dropwise at room temperature in a period time of $2 \mathrm{~h}$. This mixture was heated to $50{ }^{\circ} \mathrm{C}$ for $4 \mathrm{~h}$ and the reaction was cooled to room temperature. The yield $(\sim 80 \%)$ of bis-zinc reagent was determined by GC (after quenching with water), then the cloudy bis-zinc reagent was diluted into $0.3 \mathrm{M}$ with anhydrous $\mathrm{THF}^{t} \mathrm{BuPCl}_{2}(1.27 \mathrm{~g}$, $8 \mathrm{mmol})$ in $27 \mathrm{~mL}$ anhydrous THF $(0.3 \mathrm{M})$ was mixed with bis-zinc reagent $(0.3 \mathrm{M})$ dropwise in a $100 \mathrm{~mL}$ Schlenk flask at $0{ }^{\circ} \mathrm{C}$. The reaction was allowed to warm to room temperature slowly while stirring overnight. Sulfur powder ( $1 \mathrm{~g}, 32 \mathrm{mmol}$ ) was added under argon and the mixture was stirring for additional $3 \mathrm{~h}$.

The reaction was quenched with $50 \mathrm{~mL} \mathrm{NH}_{4} \mathrm{Cl}$ (aq.), and then extracted with $30 \mathrm{~mL}$ ethyl acetate for 3 times, the combined organic phase was washed by $100 \mathrm{~mL}$ saturated $\mathrm{NaCl}$ (aq.) and dried on anhydrous $\mathrm{NaSO}_{4}$, the solvent was removed via rotary evaporator and the residue was mixed with $10 \mathrm{~mL}{ }^{n} \mathrm{Bu}_{4} \mathrm{NF}(1 \mathrm{M}$ in $\mathrm{THF})$, the mixture was stirring for $1 \mathrm{~h}$, and solvent was removed in vacuo, the residue passed through a flash chromatography on silica (PE:EA=5:1, v/v) to afford a white powder product $(510 \mathrm{mg}, 22 \%)$. The ${ }^{1} \mathrm{H}$ NMR, ${ }^{31} \mathrm{P}$ NMR spectra matched with literature values ${ }^{3} .{ }^{1} \mathrm{H}$ NMR $\left(400 \mathrm{MHz}, \mathrm{CDCl}_{3}\right) \delta 2.50(\mathrm{ddd}, J=14.9,6.6,2.7 \mathrm{~Hz}, 1 \mathrm{H}), \delta 2.04-1.70(\mathrm{~m}, 7 \mathrm{H}), \delta 1.58$ $(\mathrm{td}, J=15.9,15.4,11.8 \mathrm{~Hz}, 1 \mathrm{H}), \delta 1.23(\mathrm{~m}, 13 \mathrm{H}), \delta 1.11-0.99(\mathrm{~m}, 1 \mathrm{H}) .{ }^{31} \mathrm{P} \mathrm{NMR}\left(162 \mathrm{MHz}, \mathrm{CDCl}_{3}\right) \delta 75.67$.

\subsection{Synthesis of $(1 R, 2 R, 3 a R, 7 a R)$-2-(tert-butyl)octahydroisophosphindole-1-carbaldehyde 2-sulfide (4)}

To a solution of $N, N, N^{\prime}, N^{\prime}$-tetramethylethylenediamine $\left(0.78 \mathrm{~mL}, 5.2 \mathrm{mmol}, 1.2\right.$ equiv.) in $\mathrm{Et}_{2} \mathrm{O}(9 \mathrm{~mL})$ was added ${ }^{s} \mathrm{BuLi}\left(4 \mathrm{~mL}, 1.3 \mathrm{M}\right.$ in hexane, $5.2 \mathrm{mmol}, 1.2$ equiv.) dropwise at $-78{ }^{\circ} \mathrm{C}$. After stirring for $30 \mathrm{~min}$, a solution of compound 3 ( $1 \mathrm{~g}, 4.35 \mathrm{mmol}, 1$ equiv.) in toluene $(6 \mathrm{~mL})$ was added dropwise. The reaction mixture was then stirred at $-78^{\circ} \mathrm{C}$ for $5 \mathrm{~h} . \mathrm{N}, \mathrm{N}$-dimethylformamide $\left(1.4 \mathrm{~mL}, 17.5 \mathrm{mmol}, 4\right.$ equiv.) was added at $-78{ }^{\circ} \mathrm{C}$ and the reaction was warmed to room temperature and stirred overnight. The reaction was quenched by water and extracted by $20 \mathrm{~mL}$

of ethyl acetate for three times. The combined organic phase was dried over anhydrous $\mathrm{Na}_{2} \mathrm{SO}_{4}$ and concentrated in vасио. The residue was purified with a flash chromatography on silica (PE:EA=5:1, v/v) to afford compound 4 as a white solid $(646 \mathrm{mg}, 58 \%) \cdot[\alpha]_{\mathrm{D}}{ }^{24}=+12.3\left(\mathrm{c}=0.13, \mathrm{CHCl}_{3}\right),{ }^{1} \mathrm{H} \mathrm{NMR}\left(400 \mathrm{MHz}, \mathrm{CDCl}_{3}\right) \delta 9.64(\mathrm{~d}, J=3.7 \mathrm{~Hz}, 1 \mathrm{H})$, $2.70(\mathrm{ddd}, J=12.6,9.2,3.7 \mathrm{~Hz}, 1 \mathrm{H}), 2.60(\mathrm{ddd}, J=14.9,6.9,1.6 \mathrm{~Hz}, 1 \mathrm{H}), 2.32(\mathrm{qdd}, J=11.6,3.5,1.7 \mathrm{~Hz}, 1 \mathrm{H}), 2.03$ $-1.89(\mathrm{~m}, 2 \mathrm{H}), 1.89-1.74(\mathrm{~m}, 3 \mathrm{H}), 1.65(\mathrm{~s}, 1 \mathrm{H}), 1.40-1.24(\mathrm{~m}, 13 \mathrm{H}), 1.05(\mathrm{qd}, J=12.5,3.4 \mathrm{~Hz}, 1 \mathrm{H}) .{ }^{13} \mathrm{C} \mathrm{NMR}(101$ $\left.\mathrm{MHz}, \mathrm{CDCl}_{3}\right) \delta 200.2,54.9(\mathrm{~d}, J=37.3 \mathrm{~Hz}), 44.9(\mathrm{~d}, J=6.6 \mathrm{~Hz}), 44.4,38.8(\mathrm{~d}, J=46.2 \mathrm{~Hz}), 34.7(\mathrm{~d}, J=42.7 \mathrm{~Hz})$, $33.3(\mathrm{~d}, J=14.5 \mathrm{~Hz}), 31.4(\mathrm{~d}, J=12.4 \mathrm{~Hz}), 25.7,25.5,24.5(\mathrm{~d}, J=2.5 \mathrm{~Hz}) .{ }^{31} \mathrm{P} \mathrm{NMR}\left(162 \mathrm{MHz} \mathrm{CDCl}_{3}\right) \delta 77.56$. HRMS (ESI-TOF) m/z: [M+H+ $\mathrm{H}^{+}$Calcd for $\mathrm{C}_{13} \mathrm{H}_{23} \mathrm{OP}=227.1559$; Found 227.1555. 


\subsection{Synthesis of $\left(1 R, 1^{\prime} R, 2 R, 2^{\prime} R, 3 a R, 3 a^{\prime} R, 7 a R, 7 a^{\prime} R\right)-1,1^{\prime}$-(azanediylbis(methylene))bis(2-(tert-}

butyl)octahydroisophosphindole 2 -sulfide) (5)

This compound was synthesized according to literature ${ }^{4}$. A slurry of the compound 4 (300 $\mathrm{mg}, 1.17 \mathrm{mmol}$ ), $\mathrm{Ti}\left(\mathrm{O}^{i} \mathrm{Pr}\right)_{4}(0.7 \mathrm{~mL}, 2.34 \mathrm{mmol}), \mathrm{NH}_{4} \mathrm{Cl}(125 \mathrm{mg}, 2.34 \mathrm{mmol})$ and triethylamine $(0.32 \mathrm{~mL}, 2.34 \mathrm{mmol})$ in anhydrous EtOH (3 mL) was stirred in a 25-mL Schlenk flask under argon at room temperature for 6 h. $\mathrm{NaBH}_{4}(90 \mathrm{mg}, 2.34$ mmol) was then added and the resulting mixture was stirred at room temperature for additional $3 \mathrm{~h}$. The reaction was then quenched by ammonium hydroxide $(2 \mathrm{M}$ aq., $3 \mathrm{~mL})$. The resulting precipitate was filtered off and washed with ethyl acetate $(3 \mathrm{~mL})$ for three times, the aqueous layer was extracted with ethyl acetate $(3 \mathrm{~mL})$ for twice. The combined organic phase was dried over anhydrous $\mathrm{Na}_{2} \mathrm{SO}_{4}$, concentrated in vacuo and purified by flash chromatography on silica (PE:EA=1:1, v/v) to afford the titled product as an off-white fluffy powder $(229 \mathrm{mg}, 78.6 \%) .[\alpha]_{\mathrm{D}}^{24}=-34.74(\mathrm{c}$ $\left.=0.19, \mathrm{CHCl}_{3}\right),{ }^{1} \mathrm{H}$ NMR $\left(400 \mathrm{MHz}, \mathrm{CDCl}_{3}\right) \delta 3.09(\mathrm{ddd}, J=15.2,12.3,7.2 \mathrm{~Hz}, 2 \mathrm{H}), \delta 2.77(\mathrm{ddd}, J=21.6,12.3,4.4$ $\mathrm{Hz}, 2 \mathrm{H}), \delta 2.50(\mathrm{ddd}, J=14.8,6.5,3.3 \mathrm{~Hz}, 2 \mathrm{H}), \delta 2.18-2.07(\mathrm{~m}, 2 \mathrm{H}), \delta 1.95-1.62(\mathrm{~m}, 10 \mathrm{H}), \delta 1.56(\mathrm{ddd}, J=16.3$, 14.8, $12.4 \mathrm{~Hz}, 2 \mathrm{H}), \delta 1.39$ (qdd, $J=11.4,3.3,1.7 \mathrm{~Hz}, 2 \mathrm{H}), \delta 1.31-114(\mathrm{~m}, 22 \mathrm{H}), \delta 1.13-0.97(\mathrm{~m}, 4 \mathrm{H}) .{ }^{13} \mathrm{C} \mathrm{NMR}$ $\left(101 \mathrm{MHz}, \mathrm{CDCl}_{3}\right) \delta 49.3,48.3(\mathrm{~d}, J=7.9 \mathrm{~Hz}), 44.0(\mathrm{~d}, J=2.4 \mathrm{~Hz}), 42.9(\mathrm{~d}, J=45.1 \mathrm{~Hz}), 37.8(\mathrm{~d}, J=47.1 \mathrm{~Hz}), 33.6$ $(\mathrm{d}, J=42.9 \mathrm{~Hz}), 33.5(\mathrm{~d}, J=14.1 \mathrm{~Hz}), 31.9(\mathrm{~d}, J=13.7 \mathrm{~Hz}), 26.0,25.9,25.2(\mathrm{~d}, J=2.5 \mathrm{~Hz}) .{ }^{31} \mathrm{P} \mathrm{NMR}(162 \mathrm{MHz}$, $\left.\mathrm{CDCl}_{3}\right) \delta$ 80.38. HRMS (ESI-TOF) m/z: [M+H'] Calcd for $\mathrm{C}_{26} \mathrm{H}_{49} \mathrm{NP}_{2} \mathrm{~S}_{2}=$ 502.2854; Found 502.2855.

\subsection{Synthesis of $b i s(((1 R, 2 S, 3 a R, 7 a R)-2$-(tert-butyl)octahydro-1H-isophosphindol-1-yl)methyl)amine (Heng- PNP)}

In a 10-mL Schlenk tube equipped with a stirring bar, a solution of Compound 5 (250 mg, $0.5 \mathrm{mmol}$ ) and $\mathrm{P}\left(\mathrm{NMe}_{2}\right)_{3}\left(1 \mathrm{~mL}, 5 \mathrm{mmol}, 10\right.$ equiv.) in degassed toluene $(3 \mathrm{~mL})$ was heated to $130{ }^{\circ} \mathrm{C}$ (oil bath) for $60 \mathrm{~h}$. The reaction was cooled to room temperature, the solvent was removed in vacuo. The residue was filtered through a silica gel plug $(\mathrm{PE}: \mathrm{EA}=1: 1, \mathrm{v} / \mathrm{v})$ under argon. $3 \mathrm{~mL}$ degassed hexane/ethyl acetate $(1: 1, \mathrm{v} / \mathrm{v})$ mixture was used to wash the stationary phase. The filtrate was concentrated in vacuo and dissolved in a minimum volume of degassed ethanol. Then 3-4 mL degassed water was added, the mixture was standing in the capped tube overnight. A white solid was formed and filtered quickly under argon. The white solid was collected and dried under vacuum (200 mg, 91.7\%). ${ }^{1} \mathrm{H}$ NMR (400 $\left.\mathrm{MHz}, \mathrm{C}_{6} \mathrm{D}_{6}\right) \delta 3.07(\mathrm{ddd}, J=15.6,11.3,4.4 \mathrm{~Hz}, 2 \mathrm{H}), 2.97(\mathrm{ddd}, J=13.4,11.3,6.7 \mathrm{~Hz}, 2 \mathrm{H}), 2.09(\mathrm{~d}, J=12.5 \mathrm{~Hz}, 2 \mathrm{H})$, $1.81(\mathrm{ddd}, J=10.3,6.9,4.1 \mathrm{~Hz}, 4 \mathrm{H}), 1.73-1.55(\mathrm{~m}, 6 \mathrm{H}), 1.41-1.16(\mathrm{~m}, 10 \mathrm{H}), 1.06(\mathrm{~d}, J=11.5 \mathrm{~Hz}, 18 \mathrm{H}), 0.90(\mathrm{q}$, $J=12.5,11.2 \mathrm{~Hz}, 4 \mathrm{H}) .{ }^{13} \mathrm{C} \mathrm{NMR}\left(101 \mathrm{MHz}, \mathrm{C}_{6} \mathrm{D}_{6}\right) \delta 52.5(\mathrm{~d}, J=26.4 \mathrm{~Hz}), 48.6,46.3(\mathrm{~d}, J=3.3 \mathrm{~Hz}), 44.0(\mathrm{~d}, J=$ 
$16.9 \mathrm{~Hz}), 33.8,31.4(\mathrm{~d}, J=3.9 \mathrm{~Hz}), 27.5(\mathrm{~d}, J=6.0 \mathrm{~Hz}), 27.4,27.3,27.2$, 25.4 (d, $J=11.6 \mathrm{~Hz}) .{ }^{31} \mathrm{P}$ NMR $(162$ MHz, $\left.\mathrm{C}_{6} \mathrm{D}_{6}\right) \delta$ 8.12. HRMS (ESI-TOF) m/z: $\left[\mathrm{M}+\mathrm{H}^{+}\right]$Calcd for $\mathrm{C}_{26} \mathrm{H}_{49} \mathrm{NP}_{2}=438.3413$; Found 438.3409.

\subsection{Synthesis of $\left[\operatorname{Ir}(\mathrm{COE})_{2} \mathrm{Cl}\right]_{2}$}

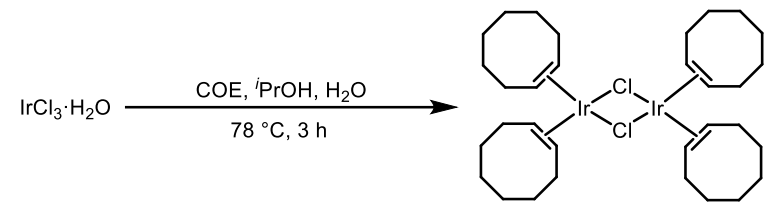

$\left[\operatorname{Ir}(\mathrm{COE})_{2} \mathrm{Cl}\right]_{2}$ was synthesized with a minor-modified method ${ }^{5}$. A $50-\mathrm{mL}$ three-necked round bottled flask was charged with a stirring bar, $750 \mathrm{mg}$ of $\mathrm{IrCl}_{3} \cdot \mathrm{xH}_{2} \mathrm{O}, 9 \mathrm{~mL}$ of ${ }^{i} \mathrm{PrOH}, 3.4 \mathrm{~mL}$ of $\mathrm{H}_{2} \mathrm{O}$ and $1.67 \mathrm{~mL}$ of 1-cyclooctene. The flask was equipped with a condenser and the whole system was degassed by freeze-pump-thaw. The reaction was refluxed (in an oil bath) for $3 \mathrm{~h}$, then the mixture was allowed to cool to room temperature. The $\left[\operatorname{Ir}(\mathrm{COE})_{2} \mathrm{Cl}\right]_{2}$ was collected by quickly filtration and washed with ice-cold methanol, dried under vacuum to afford a yellow powder $(1.27 \mathrm{~g}, 60 \%)$. The characterization data were in agreement with literature.

\subsection{Synthesis of $\left[\mathrm{IrH}\left(\mathrm{C}_{8} \mathrm{H}_{13}\right) \mathrm{Cl}(\right.$ Heng-PNP) $]$ (Ir/Heng-PNP)}

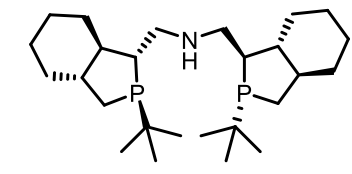

Heng-PNP

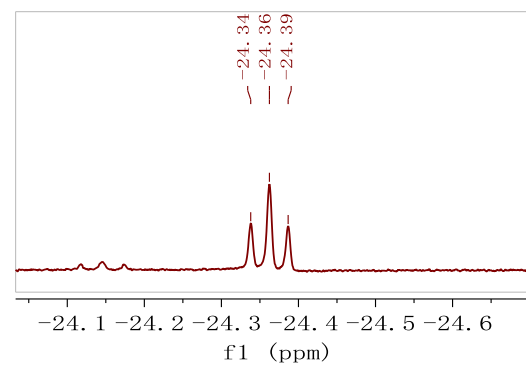

${ }^{1} \mathrm{H} \mathrm{NMR}\left(600 \mathrm{MHz}, \mathrm{C}_{6} \mathrm{D}_{6}\right)$

Ir-H: $\delta-24.18(\mathrm{t}, \mathrm{J}=14.6 \mathrm{~Hz}, 1 \mathrm{H})$
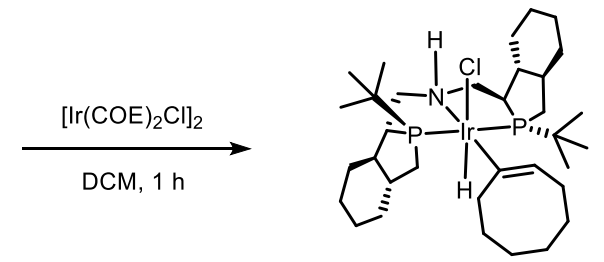

precatalyst

Full conversion

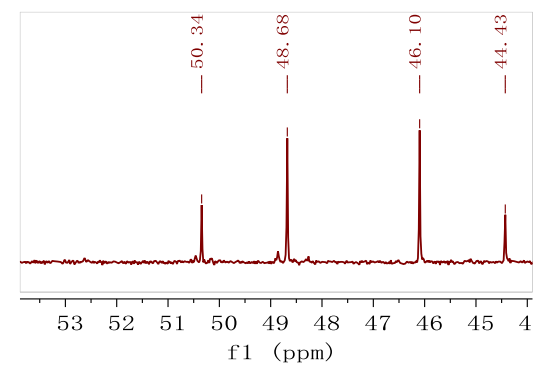

${ }^{31} \mathrm{P}\left\{{ }^{1} \mathrm{H}\right\} \mathrm{NMR}\left(243 \mathrm{MHz}, \mathrm{C}_{6} \mathrm{D}_{6}\right)$ :

$\delta 49.51(\mathrm{~d}, \mathrm{~J}=405.5 \mathrm{~Hz})$

$\delta 45.26(\mathrm{~d}, \mathrm{~J}=405.4 \mathrm{~Hz})$.

A solution of $\left[\mathrm{Ir}(\mathrm{COE})_{2} \mathrm{Cl}\right]_{2}(10.8 \mathrm{mg}, 12 \mu \mathrm{mol}, 1$ equiv. $)$ and $\mathrm{L}(10.8 \mathrm{mg}, 24.72 \mu \mathrm{mol}, 2.06$ equiv. $)$ in degassed dichloromethane $(1.2 \mathrm{~mL})$ was stirring under argon at room temperature for $1 \mathrm{~h}$ in a $10 \mathrm{~mL}$ Schlenk tube. The mixture 
passed through celite and was concentrated in vacuo to afford a yellow solid (18.2 mg, 98\%). This crude product was pure enough for further use.

${ }^{1} \mathrm{H}$ NMR, ${ }^{31} \mathrm{P}$ NMR and high-resolution mass spectroscopy analysis indicated an $\operatorname{Ir}(\mathrm{III})$ monohydride complex with a X-type COE ligand. A C-H activation occurs after the coordination of PNP ligand to $\operatorname{Ir}(\mathrm{I})$ precursor. (Scheme 3) In addition, two sets of doublet peaks in ${ }^{31} \mathrm{P}$ NMR suggested that two different phosphorus atoms. Similar reports were observed in literatures ${ }^{6} .{ }^{1} \mathrm{H}$ NMR $\left(600 \mathrm{MHz}, \mathrm{C}_{6} \mathrm{D}_{6}\right) \delta 4.97(\mathrm{~s}, 1 \mathrm{H}), 3.27(\mathrm{t}, J=13.5 \mathrm{~Hz}, 1 \mathrm{H}), 2.22$ (dddd, $J=55.8$, 43.2, 17.5, $12.0 \mathrm{~Hz}, 4 \mathrm{H}), 1.92(\mathrm{~s}, 1 \mathrm{H}), 1.68(\mathrm{~s}, 1 \mathrm{H}), 1.65-1.40(\mathrm{~m}, 12 \mathrm{H}), 1.34(\mathrm{dt}, J=19.3,9.3 \mathrm{~Hz}, 6 \mathrm{H}), 1.21(\mathrm{~d}, J=$ $13.4 \mathrm{~Hz}, 9 \mathrm{H}), 1.18-1.08(\mathrm{~m}, 7 \mathrm{H}), 1.08-1.01(\mathrm{~m}, 6 \mathrm{H}), 0.98(\mathrm{~d}, J=13.3 \mathrm{~Hz}, 9 \mathrm{H}), 0.93-0.81(\mathrm{~m}, 4 \mathrm{H}), 0.75(\mathrm{q}, J=$ 15.0, $13.4 \mathrm{~Hz}, 4 \mathrm{H}), 0.68(\mathrm{t}, J=7.2 \mathrm{~Hz}, 2 \mathrm{H}), 0.61-0.36(\mathrm{~m}, 10 \mathrm{H}),-24.36(\mathrm{t}, J=14.5 \mathrm{~Hz}, 1 \mathrm{H}) .{ }^{31} \mathrm{P}$ NMR $(243 \mathrm{MHz}$, $\left.\mathrm{C}_{6} \mathrm{D}_{6}\right) \delta 49.51(\mathrm{~d}, J=405.5 \mathrm{~Hz}), 45.26(\mathrm{~d}, J=405.4 \mathrm{~Hz})$. HRMS (ESI-TOF) m/z: [M-Cl-] Calcd for $\mathrm{C}_{34} \mathrm{H}_{63} \mathrm{ClIrNP}_{2}=$ 740.4059 Found 740.4043. A triplet signal $(\delta-24.14, \sim 2 \%)$ was observed in the $1 \mathrm{H}$ NMR spectrum, which probably indicates another diastereomer. 


\section{Synthesis of biaryl ketones.}

Some diarylketones were commercially available (9a-9c), others were prepared as described in literature ${ }^{[7]}$ for substrates 9d-9w:

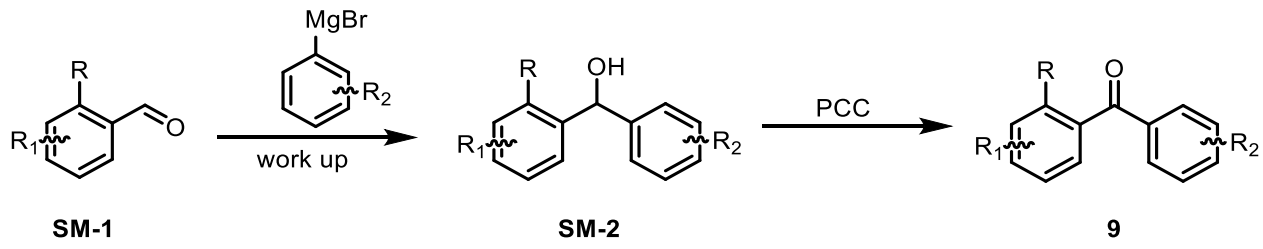

To a stirred solution of aryl aldehydes SM-1 (1.0 equivalent) in a dry THF at $-40{ }^{\circ} \mathrm{C}$ (in a chiller) under argon a solution of arylmagnesium bromide (1.2 equivalent) was added dropwise. After finish, the reaction mixture was stirred about $0.5 \mathrm{~h}$ at $-40{ }^{\circ} \mathrm{C}$. Then the mixture was allowed to worm to room temperature and stirred for $4 \mathrm{~h}$. After that, the reaction was quenched by adding a saturated aqueous solution of ammonium chloride. Then the product was extracted with ethyl acetate and the combined organic layer was dried over sodium sulfate. After the solvent was evaporated in vacuo, the crude product was obtained for further reaction without purification. To a stirred solution of diarylalcohol SM-2 (1.0 equivalent) in DCM (5mL/mmoL) at room temperature was added a homogeneous mixture of PCC (3.0 equivalent) and silica gel (1:1 by mass,). The mixture was stirred at room temperature for $2 \mathrm{~h}$, then the mixture was filtered through a pad of silica gel which was washed with DCM twice. The filtrate was then concentrated, the product was purified by flash chromatography (on silica, petroleum ether : ethyl acetate $=10: 1, \mathrm{v} / \mathrm{v}$ ).<smiles>O=C(c1ccccc1)c1ccccc1I</smiles>

(2-iodophenyl)(phenyl)methanone ${ }^{3}(9 \mathrm{c})$ Purified by column chromatography ( silica gel, PE:EA=10:1, v/v), Colorless oil, $813 \mathrm{mg}, 66 \%$ yield, ${ }^{1} \mathrm{H}$ NMR $\left(600 \mathrm{MHz}, \mathrm{CDCl}_{3}\right) \delta 7.92(\mathrm{dd}, J=8.0,1.1 \mathrm{~Hz}, 1 \mathrm{H})$, $7.81(\mathrm{dd}, J=8.2,1.4 \mathrm{~Hz}, 2 \mathrm{H}), 7.62-7.57(\mathrm{~m}, 1 \mathrm{H}), 7.48-7.42(\mathrm{~m}, 3 \mathrm{H}), 7.29(\mathrm{dd}, J=7.6,1.7 \mathrm{~Hz}, 1 \mathrm{H}), 7.18(\mathrm{td}, J=$ 7.7, $1.7 \mathrm{~Hz}, 1 \mathrm{H}) .{ }^{13} \mathrm{C} \mathrm{NMR}\left(151 \mathrm{MHz}, \mathrm{CDCl}_{3}\right) \delta 197.3,144.4,139.7,135.6,133.8,131.2,130.5,128.7,128.5$, $127.8,92.3,77.3,77.1,76.9$.<smiles>O=C(c1ccccc1)c1ccccc1C(F)(F)F</smiles>

Phenyl(2-(trifluoromethyl)phenyl)methanone ${ }^{2}$ (9e) Purified by column chromatography ( silica gel, PE:EA=10:1, v/v), Colorless oil, $650 \mathrm{mg}, 65 \%$ yield, ${ }^{1} \mathrm{H}$ NMR $\left(400 \mathrm{MHz}, \mathrm{CDCl}_{3}\right) \delta 7.82-7.76(\mathrm{~m}, 3 \mathrm{H})$, $7.66-7.58(\mathrm{~m}, 3 \mathrm{H}), 7.49-7.43(\mathrm{~m}, 2 \mathrm{H}), 7.41-7.37(\mathrm{~m}, 1 \mathrm{H}) .{ }^{13} \mathrm{C} \mathrm{NMR}\left(101 \mathrm{MHz}, \mathrm{CDCl}_{3}\right) \delta 195.6,138.3(\mathrm{q}, J=$ $2.1 \mathrm{~Hz}), 136.4,133.9,131.5,130.2,129.9,128.6,128.1,128.0(\mathrm{q}, J=32.3 \mathrm{~Hz}), 126.7(\mathrm{q}, J=4.7 \mathrm{~Hz}), 123.7(\mathrm{q}, J=$ $274.0 \mathrm{~Hz})$. 
<smiles>O=C(c1ccccc1)c1ccccc1-c1ccccc1</smiles>

[1,1'-biphenyl]-2-yl(phenyl)methanone ${ }^{4}(9 \mathrm{f})$ Purified by column chromatography ( silica gel, PE:EA=10:1, v/v), White solid, 72\% yield, ${ }^{1} \mathrm{H}$ NMR $\left(400 \mathrm{MHz}, \mathrm{CDCl}_{3}\right) \delta 7.64(\mathrm{~d}, J=7.8 \mathrm{~Hz}, 2 \mathrm{H}), 7.60-7.55$ (m, 1H), 7.45 (ddt, $J=30.7 \mathrm{~Hz}, 14.8 \mathrm{~Hz}, 7.3 \mathrm{~Hz}, 4 \mathrm{H}), 7.26(\mathrm{t}, J=7.1 \mathrm{~Hz}, 4 \mathrm{H}), 7.18(\mathrm{dt}, J=12.8 \mathrm{~Hz}, 6.9 \mathrm{~Hz}, 3 \mathrm{H}) .{ }^{13} \mathrm{C}$ NMR (101 MHz, $\left.\mathrm{CDCl}_{3}\right) \delta$ 198.8, 141.2, 140.2, 139.0, 137.4, 132.8, 130.4, 130.1, 129.9, 129.0, 128.8, 128.3, 128.1, 127.3, 127.1.<smiles>O=C(c1ccccc1)c1cccc2ccccc12</smiles>

naphthalen-1-yl(phenyl)methanone ${ }^{13}(9 \mathrm{~g})$ Purified by column chromatography ( silica gel, PE:EA=10:1, v/v), Yellow oil, $525 \mathrm{mg}, 72 \%$ yield, ${ }^{1} \mathrm{H}$ NMR $\left(400 \mathrm{MHz}, \mathrm{CDCl}_{3}\right) \delta 8.58-8.51(\mathrm{~m}, 1 \mathrm{H}), 8.00(\mathrm{~d}, J=$ $8.1 \mathrm{~Hz}, 1 \mathrm{H}), 7.93-7.88(\mathrm{~m}, 1 \mathrm{H}), 7.68(\mathrm{td}, J=7.4,1.9 \mathrm{~Hz}, 1 \mathrm{H}), 7.64(\mathrm{~d}, J=7.0 \mathrm{~Hz}, 1 \mathrm{H}), 7.61-7.49(\mathrm{~m}, 3 \mathrm{H}), 7.45$ $(\mathrm{t}, J=7.7 \mathrm{~Hz}, 1 \mathrm{H}), 7.24(\mathrm{td}, J=7.6,1.3 \mathrm{~Hz}, 1 \mathrm{H}), 7.11(\mathrm{ddd}, J=9.6,8.3,1.0 \mathrm{~Hz}, 1 \mathrm{H}) .{ }^{13} \mathrm{C} \mathrm{NMR}\left(151 \mathrm{MHz}, \mathrm{CDCl}_{3}\right)$ $\delta 194.9,161.8,160.1,136.1,134.0,133.9,133.9,132.9,131.6,130.7,129.9,128.5,128.3,128.2,128.0,126.6$, 125.6, 124.4, 124.3, 124.3, 116.7, 116.5 .<smiles>O=C(c1ccoc1)c1ccccc1Br</smiles>

(2-bromophenyl)(furan-3-yl)methanone ${ }^{14}$ (9h) Purified by column chromatography ( silica gel, PE:EA=10:1, v/v), Yellow oil, $248 \mathrm{mg}, 65 \%$ yield, ${ }^{1} \mathrm{H}$ NMR $\left(600 \mathrm{MHz}, \mathrm{CDCl}_{3}\right) \delta 7.69-7.67(\mathrm{~m}, 1 \mathrm{H}), 7.67-$ $7.63(\mathrm{~m}, 1 \mathrm{H}), 7.49(\mathrm{t}, J=1.7 \mathrm{~Hz}, 1 \mathrm{H}), 7.42-7.37(\mathrm{~m}, 2 \mathrm{H}), 7.36-7.31(\mathrm{~m}, 1 \mathrm{H}), 6.88(\mathrm{~d}, J=2.0 \mathrm{~Hz}, 1 \mathrm{H}) .{ }^{13} \mathrm{C} \mathrm{NMR}$ $\left(151 \mathrm{MHz}, \mathrm{CDCl}_{3}\right) \delta 189.2,150.4,144.6,141.1,133.6,131.4,128.5,127.4,127.2,119.2,109.2$.

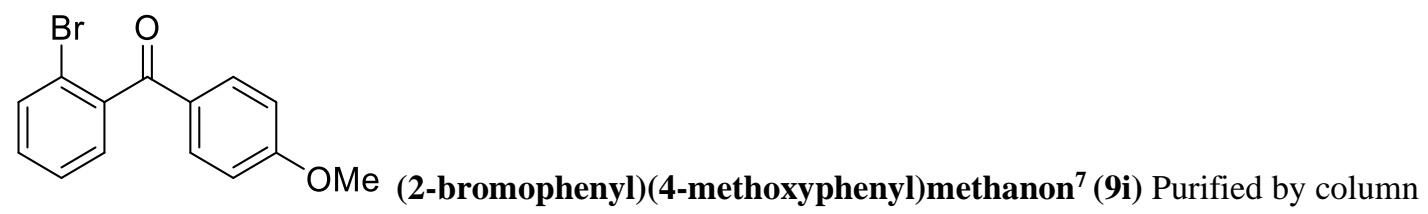

chromatography ( silica gel, PE:EA=10:1, v/v), White solid, $142 \mathrm{mg}, 60 \%$ yield, ${ }^{1} \mathrm{H} \mathrm{NMR}\left(400 \mathrm{MHz}, \mathrm{CDCl}_{3}\right) \delta 7.81$ $-7.77(\mathrm{~m}, 2 \mathrm{H}), 7.64(\mathrm{dd}, J=8.2,1.2 \mathrm{~Hz}, 1 \mathrm{H}), 7.44-7.38(\mathrm{~m}, 1 \mathrm{H}), 7.36-7.31(\mathrm{~m}, 2 \mathrm{H}), 6.96-6.91(\mathrm{~m}, 2 \mathrm{H}), 3.88$ (s, 3H). ${ }^{13} \mathrm{C} \mathrm{NMR}\left(101 \mathrm{MHz}, \mathrm{CDCl}_{3}\right) \delta 194.5,164.2,141.1,133.1,132.7,130.9,129.1,128.8,127.2,119.4,113.9$, 55.6 . 
(2-bromophenyl)(p-tolyl)methanone ${ }^{7}$ (9j) Purified by column chromatography ( silica gel, PE:EA=10:1, v/v), White solid, $354 \mathrm{mg}, 64 \%$ yield, ${ }^{1} \mathrm{H}$ NMR (600 MHz, $\left.\mathrm{CDCl}_{3}\right) \delta 7.73-7.69(\mathrm{~m}, 2 \mathrm{H}), 7.64$ (dd, $J$ $=8.0,1.1 \mathrm{~Hz}, 1 \mathrm{H}), 7.41(\mathrm{td}, J=7.4,1.2 \mathrm{~Hz}, 1 \mathrm{H}), 7.36-7.31(\mathrm{~m}, 2 \mathrm{H}), 7.28-7.25(\mathrm{~m}, 2 \mathrm{H}), 2.43(\mathrm{~s}, 3 \mathrm{H}) .{ }^{13} \mathrm{C} \mathrm{NMR}$ $\left(151 \mathrm{MHz}, \mathrm{CDCl}_{3}\right) \delta$ 195.6, 144.8, 141.0, 133.6, 133.1, 131.0, 130.4, 129.4, 128.9, 127.2, 119.5, 21.8.<smiles>O=C(c1ccc(Cl)cc1)c1ccccc1Br</smiles>

$\mathrm{Cl}_{\text {(2-bromophenyl)(4-chlorophenyl)methanone }}{ }^{7}$ (9k) Purified by column chromatography ( silica gel, PE:EA=10:1, v/v), White solid, $850 \mathrm{mg}, 72 \%$ yield, ${ }^{1} \mathrm{H}$ NMR (400 MHz, $\left.\mathrm{CDCl}_{3}\right) \delta 7.77-7.73(\mathrm{~m}, 2 \mathrm{H})$, $7.65(\mathrm{dd}, J=7.8,1.2 \mathrm{~Hz}, 1 \mathrm{H}), 7.46-7.41(\mathrm{~m}, 3 \mathrm{H}), 7.40-7.32(\mathrm{~m}, 2 \mathrm{H}) .{ }^{13} \mathrm{C}$ NMR $\left(101 \mathrm{MHz}, \mathrm{CDCl}_{3}\right) \delta 194.7$, $140.4,140.2,134.5,133.3,131.5,131.4,129.1,129.0,127.4,119.5$.<smiles>O=C(c1ccc(F)cc1)c1ccccc1Br</smiles>

(2-bromophenyl)(4-fluorophenyl)methanone ${ }^{7}$ (9l) Purified by column chromatography ( silica gel, PE:EA=10:1, v/v), Yellow oil, $926 \mathrm{mg}, 83 \%$ yield, ${ }^{1} \mathrm{H}$ NMR $\left(400 \mathrm{MHz}, \mathrm{CDCl}_{3}\right) \delta 7.87-7.80(\mathrm{~m}, 2 \mathrm{H})$, $7.64(\mathrm{dd}, J=7.9,1.2 \mathrm{~Hz}, 1 \mathrm{H}), 7.41(\mathrm{dd}, J=7.4,1.2 \mathrm{~Hz}, 1 \mathrm{H}), 7.35(\mathrm{ddd}, J=13.4,7.5,1.9 \mathrm{~Hz}, 2 \mathrm{H}), 7.16-7.09$ (m, $2 \mathrm{H}) .{ }^{13} \mathrm{C}$ NMR $\left(101 \mathrm{MHz}, \mathrm{CDCl}_{3}\right) \delta 194.3,166.2(\mathrm{~d}, J=257.6 \mathrm{~Hz}), 140.4,133.3,133.0,132.9,132.6(\mathrm{~d}, J=2.0$ Hz), 131.3, 128.9, 127.4, 119.4, 116.0, 115.8 .

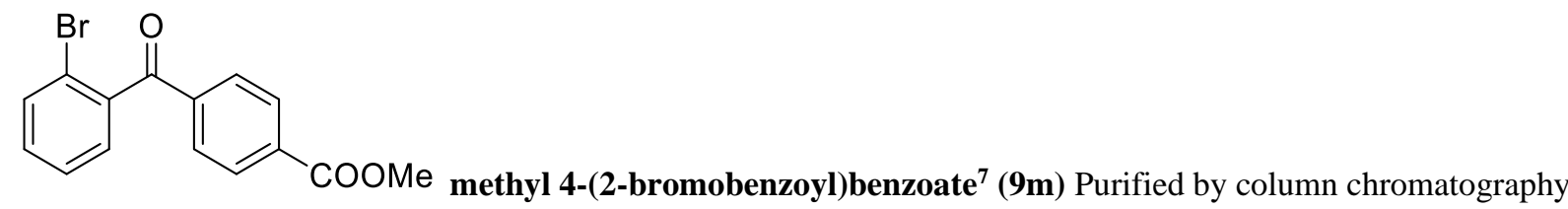
( silica gel, PE:EA=10:1, v/v), White solid, $1.31 \mathrm{~g}, 72 \%,{ }^{1} \mathrm{H}$ NMR $\left(600 \mathrm{MHz}, \mathrm{CDCl}_{3}\right) \delta 8.12(\mathrm{~d}, J=8.3 \mathrm{~Hz}, 2 \mathrm{H})$, $7.86(\mathrm{~d}, J=8.4 \mathrm{~Hz}, 2 \mathrm{H}), 7.66(\mathrm{~d}, J=8.0 \mathrm{~Hz}, 1 \mathrm{H}), 7.45(\mathrm{dd}, J=8.0,6.9 \mathrm{~Hz}, 1 \mathrm{H}), 7.41-7.36(\mathrm{~m}, 2 \mathrm{H}), 3.95(\mathrm{~s}, 3 \mathrm{H})$. ${ }^{13} \mathrm{C}$ NMR $\left(151 \mathrm{MHz}, \mathrm{CDCl}_{3}\right) \delta 195.4,166.2,140.1,139.5,134.3,133.4,131.6,130.0,129.8,129.2,127.4,119.6$, 52.5 .<smiles>O=C(c1cccc(F)c1)c1ccccc1Br</smiles>

(2-bromophenyl)(3-fluorophenyl)methanone ${ }^{7}$ (9n) Purified by column chromatography ( silica gel, PE:EA=10:1, v/v), Yellow oil, $125 \mathrm{mg}, 62 \%$ yield, ${ }^{1} \mathrm{H}$ NMR (600 MHz, $\left.\mathrm{CDCl}_{3}\right) \delta 7.65(\mathrm{dd}, J=8.2,4.0$ 
$\mathrm{Hz}, 1 \mathrm{H}), 7.54(\mathrm{t}, J=9.8 \mathrm{~Hz}, 2 \mathrm{H}), 7.43(\mathrm{td}, J=7.7,7.2,3.8 \mathrm{~Hz}, 2 \mathrm{H}), 7.41-7.33(\mathrm{~m}, 2 \mathrm{H}), 7.33-7.26(\mathrm{~m}, 1 \mathrm{H}) .{ }^{13} \mathrm{C}$ NMR $\left(151 \mathrm{MHz}, \mathrm{CDCl}_{3}\right) \delta 194.6,162.8(\mathrm{~d}, J=249.2 \mathrm{~Hz}), 140.1,138.3(\mathrm{~d}, J=6.0 \mathrm{~Hz}), 133.3,131.5,130.4(\mathrm{~d}, J=$ 7.6 Hz), 129.0, 127.4, 126.2, $120.8(\mathrm{~d}, J=22.7 \mathrm{~Hz}), 119.5,116.5 .(\mathrm{d}, J=24.2 \mathrm{~Hz})$.<smiles>Cc1ccccc1C(=O)c1ccc(F)cc1</smiles>

(4-bromophenyl)(o-tolyl)methanone ${ }^{10}(9 p)$ Purified by column chromatography ( silica gel, PE:EA=10:1, v/v), Colorless oil, $759 \mathrm{mg}, 69 \%$ yield, ${ }^{1} \mathrm{H}$ NMR (400 MHz, $\left.\mathrm{CDCl}_{3}\right) \delta 7.59-7.50(\mathrm{~m}, 2 \mathrm{H}), 7.50-$ $7.43(\mathrm{~m}, 2 \mathrm{H}), 7.27(\mathrm{td}, J=7.4 \mathrm{~Hz}, 1.7 \mathrm{~Hz}, 1 \mathrm{H}), 7.14(\mathrm{ddd}, J=15.3 \mathrm{~Hz}, 7.5 \mathrm{~Hz}, 1.4 \mathrm{~Hz}, 3 \mathrm{H}), 2.21(\mathrm{~s}, 3 \mathrm{H}) .{ }^{13} \mathrm{C} \mathrm{NMR}$ $\left(101 \mathrm{MHz}, \mathrm{CDCl}_{3}\right) \delta$ 197.4, 138.0, 136.9, 136.5, 131.8, 131.6, 131.2, 130.6, 128.5, 128.4, 125.3, 20.1.

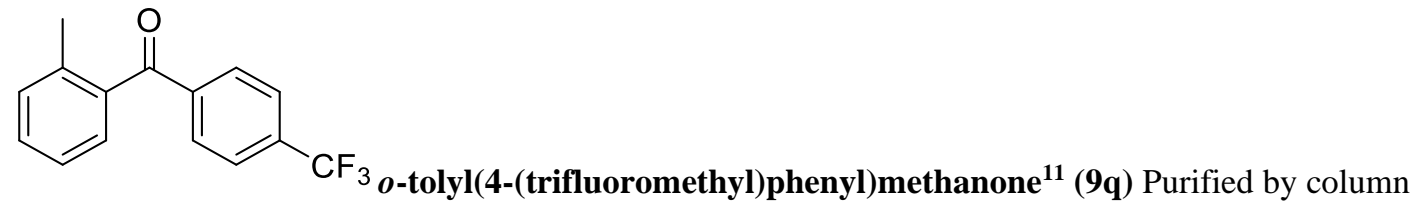
chromatography ( silica gel, PE:EA=10:1, v/v), Colorless oil, $623 \mathrm{mg}, 59 \%$ yield, ${ }^{1} \mathrm{H} \mathrm{NMR}\left(400 \mathrm{MHz}, \mathrm{CDCl}_{3}\right) \delta$ $7.82-7.72(\mathrm{~m}, 2 \mathrm{H}), 7.58(\mathrm{~d}, J=8.2 \mathrm{~Hz}, 2 \mathrm{H}), 7.29(\mathrm{td}, J=7.4 \mathrm{~Hz}, 1.7 \mathrm{~Hz}, 1 \mathrm{H}), 7.18(\mathrm{dd}, J=7.7 \mathrm{~Hz}, 1.6 \mathrm{~Hz}, 2 \mathrm{H})$, $7.12(\mathrm{td}, J=7.2 \mathrm{~Hz}, 6.7 \mathrm{~Hz}, 1.1 \mathrm{~Hz}, 1 \mathrm{H}), 2.23$ (s, 3H). ${ }^{13} \mathrm{C} \mathrm{NMR}\left(101 \mathrm{MHz}, \mathrm{CDCl}_{3}\right) \delta 197.3,140.8,137.5,137.4$, 134.2 (q, $J=32.6 \mathrm{~Hz}), 131.4,130.9,130.3,129.0,125.5$ (q, $J=3.7 \mathrm{~Hz}), 125.4,123.7$ (q, $J=272.7 \mathrm{~Hz}), 20.1$.

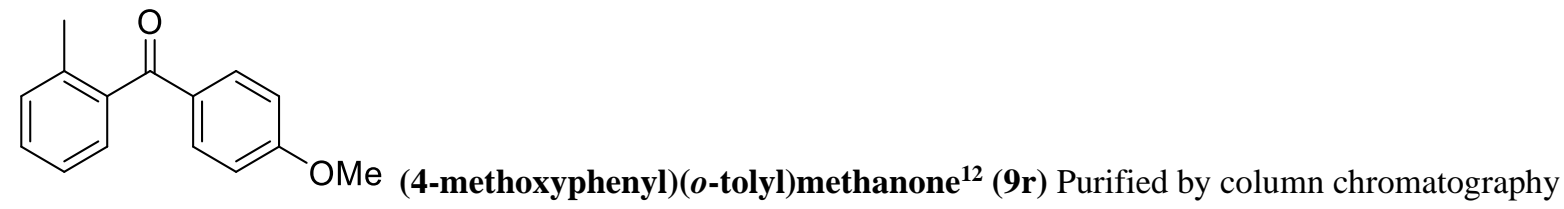
( silica gel, PE:EA=10:1, v/v), Yellow oil, $705 \mathrm{mg}, 78 \%$ yield, ${ }^{1} \mathrm{H}$ NMR $\left(400 \mathrm{MHz}, \mathrm{CDCl}_{3}\right) \delta 7.73-7.64(\mathrm{~m}, 2 \mathrm{H})$, $7.26(\mathrm{td}, J=7.3 \mathrm{~Hz}, 1.5 \mathrm{~Hz}, 1 \mathrm{H}), 7.21-7.10(\mathrm{~m}, 3 \mathrm{H}), 6.86-6.78(\mathrm{~m}, 2 \mathrm{H}), 3.75(\mathrm{~s}, 3 \mathrm{H}), 2.20(\mathrm{~s}, 3 \mathrm{H}) .{ }^{13} \mathrm{C} \mathrm{NMR}$ $\left(101 \mathrm{MHz}, \mathrm{CDCl}_{3}\right) \delta 197.4,163.7,139.2,136.2,132.5,130.8,130.5,129.8,127.9,125.2,113.7,55.5,19.8$.<smiles>O=C(c1ccccc1)c1ccc(Cl)cc1Br</smiles>

(2-bromo-4-chlorophenyl)(phenyl)methanone9 (9s) P Purified by column

chromatography ( silica gel, PE:EA=10:1, v/v), White solid, $566 \mathrm{mg}, 75 \%$ yield, ${ }^{1} \mathrm{H}$ NMR $\left(400 \mathrm{MHz}, \mathrm{CDCl}_{3}\right) \delta 7.69$ $(\mathrm{dd}, J=8.4 \mathrm{~Hz}, 1.4 \mathrm{~Hz}, 2 \mathrm{H}), 7.56(\mathrm{~d}, J=1.9 \mathrm{~Hz}, 1 \mathrm{H}), 7.54-7.46(\mathrm{~m}, 1 \mathrm{H}), 7.42-7.33(\mathrm{~m}, 2 \mathrm{H}), 7.30(\mathrm{dd}, J=8.2$ $\mathrm{Hz}, 2.0 \mathrm{~Hz}, 1 \mathrm{H}), 7.19(\mathrm{~m}, 1 \mathrm{H}) .{ }^{13} \mathrm{C} \mathrm{NMR}\left(101 \mathrm{MHz}, \mathrm{CDCl}_{3}\right) \delta 194.9,139.0,136.5,135.9,134.0,133.0,130.2$, 130.0, 128.8, 127.6, 120.3. 
<smiles>O=C(c1ccccc1Cl)c1ccccc1Cl</smiles>

(2-chlorophenyl)(2-fluorophenyl)methanone ${ }^{8}$ (9t) Purified by column chromatography ( silica gel, PE:EA=10:1, v/v), Light yellow oil, $176 \mathrm{mg} .73 \%$ yield, ${ }^{1} \mathrm{H}$ NMR $\left(400 \mathrm{MHz}, \mathrm{CDCl}_{3}\right) \delta 7.78(\mathrm{td}, J=7.5,1.9 \mathrm{~Hz}$, 1H), 7.59 (tdd, $J=6.3,5.1,2.5 \mathrm{~Hz}, 1 \mathrm{H}), 7.51-7.44$ (m, 3H), $7.42-7.36$ (m, 1H), 7.29 (s, 1H), 7.13 (ddd, $J=10.6$, 8.3, $1.0 \mathrm{~Hz}, 1 \mathrm{H}) .{ }^{13} \mathrm{C}$ NMR $\left(101 \mathrm{MHz}, \mathrm{CDCl}_{3}\right) \delta 192.2,161.6(\mathrm{~d}, J=262.6 \mathrm{~Hz}) 160.3,139.5,135.0(\mathrm{~d}, J=9.1 \mathrm{~Hz})$, $131.8,131.5,130.2,129.5,126.9,126.3(\mathrm{~d}, J=11.1 \mathrm{~Hz}), 124.5(\mathrm{~d}, J=4.0 \mathrm{~Hz}), 116.6(\mathrm{~d}, J=22.2 \mathrm{~Hz})$.<smiles>O=C(c1ccccc1F)c1ccccc1I</smiles>

(2-fluorophenyl)(2-iodophenyl)methanone ${ }^{7}$ (9u) Purified by column chromatography ( silica gel, PE:EA=10:1, v/v), Light yellow oil, $913 \mathrm{mg} .70 \%$ yield, ${ }^{1} \mathrm{H}$ NMR $(600 \mathrm{MHz}, \mathrm{CDCl}) \delta 7.91$ (d, J=8.0 Hz, $1 \mathrm{H}), 7.73(\mathrm{t}, J=7.6 \mathrm{~Hz}, 1 \mathrm{H}), 7.59-7.53(\mathrm{~m}, 1 \mathrm{H}), 7.42(\mathrm{t}, J=7.6 \mathrm{~Hz}, 1 \mathrm{H}), 7.34(\mathrm{~d}, J=7.6 \mathrm{~Hz}, 1 \mathrm{H}), 7.24(\mathrm{~d}, J=7.6$ $\mathrm{Hz}, 1 \mathrm{H}), 7.16(\mathrm{t}, J=7.8 \mathrm{~Hz}, 1 \mathrm{H}), 7.10(\mathrm{t}, J=9.5 \mathrm{~Hz}, 1 \mathrm{H}) .{ }^{13} \mathrm{C} \mathrm{NMR}\left(151 \mathrm{MHz}, \mathrm{CDCl}_{3}\right) \delta 194.1,161.8(\mathrm{~d}, J=256.7$ Hz) $160.9,144.8,140.1,135.2(\mathrm{~d}, J=9.1 \mathrm{~Hz}), 132.0,131.8,128.9,128.0,125.2(\mathrm{~d}, J=3.0 \mathrm{~Hz}), 124.5(\mathrm{~d}, J=10.6$ Hz), $116.9(\mathrm{~d}, J=21.1 \mathrm{~Hz}), 91.8,77.3,77.1,76.9$.<smiles>O=C(c1ccccc1F)c1ccccc1F</smiles>

(2-bromophenyl)(2-fluorophenyl)methanone ${ }^{7}$ (9v) Purified by column chromatography ( silica gel, PE:EA=10:1, v/v), White solid, $1.01 \mathrm{~g}, 75 \%$ yield. ${ }^{1} \mathrm{H}$ NMR $\left(600 \mathrm{MHz}, \mathrm{CDCl}_{3}\right) \delta 7.76$ (td, $J=7.6,1.8$ $\mathrm{Hz}, 1 \mathrm{H}), 7.63(\mathrm{~d}, J=8.1 \mathrm{~Hz}, 1 \mathrm{H}), 7.57$ (dtt, $J=7.0,5.0,2.5 \mathrm{~Hz}, 1 \mathrm{H}), 7.43-7.39$ (m, 2H), 7.35 (ddt, $J=8.2,6.2,3.0$ $\mathrm{Hz}, 1 \mathrm{H}), 7.26(\mathrm{~d}, J=15.1 \mathrm{~Hz}, 1 \mathrm{H}), 7.11(\mathrm{dd}, J=10.6,8.3 \mathrm{~Hz}, 1 \mathrm{H}) .{ }^{13} \mathrm{C}$ NMR $\left(101 \mathrm{MHz}, \mathrm{CDCl}_{3}\right) \delta 192.8,161.7(\mathrm{~d}$, $J=258.6 \mathrm{~Hz}), 141.6,135.2(\mathrm{~d}, J=9.1 \mathrm{~Hz}), 133.3,131.7(\mathrm{~d}, J=3.0 \mathrm{~Hz}), 129.3,127.4,125.8(\mathrm{~d}, J=10.1 \mathrm{~Hz}), 124.4$ $(\mathrm{d}, J=4.0 \mathrm{~Hz}), 119.5,116.7(\mathrm{~d}, J=22.2 \mathrm{~Hz})$.<smiles>COc1ccccc1C(=O)c1ccccc1Br</smiles>

(2-bromophenyl)(2-methoxyphenyl)methanone ${ }^{7}$ (9w) Purified by column chromatography ( silica gel, PE:EA=10:1, v/v), Light yellow oil, $913 \mathrm{mg}$. $70 \%$ yield, ${ }^{1} \mathrm{H}$ NMR $\left(600 \mathrm{MHz}, \mathrm{CDCl}_{3}\right) \delta 7.67$ (dd, $J=7.7$, $1.8 \mathrm{~Hz}, 1 \mathrm{H}), 7.59(\mathrm{dd}, J=8.0,1.1 \mathrm{~Hz}, 1 \mathrm{H}), 7.53-7.50(\mathrm{~m}, 1 \mathrm{H}), 7.38-7.34(\mathrm{~m}, 2 \mathrm{H}), 7.30-7.26(\mathrm{~m}, 1 \mathrm{H}), 7.03(\mathrm{td}$, $J=7.6,1.0 \mathrm{~Hz}, 1 \mathrm{H}), 6.95-6.92(\mathrm{~m}, 1 \mathrm{H}), 3.66(\mathrm{~s}, 3 \mathrm{H}) .{ }^{13} \mathrm{C} \mathrm{NMR}\left(151 \mathrm{MHz}, \mathrm{CDCl}_{3}\right) \delta 195.3,159.4,142.7,134.4$, $133.0,131.8,130.9,129.3,127.2,127.1,120.7,119.5,112.0,55.8$. 


\section{(4-(phenylethynyl)phenyl)(o-tolyl)methanone (9o)}

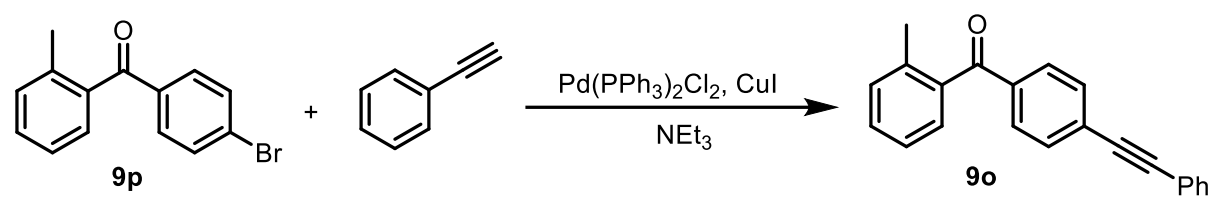

A solution of the corresponding 9p (1.0 equivalent), $\mathrm{Pd}\left(\mathrm{PPh}_{3}\right)_{2} \mathrm{Cl}_{2}(2.0 \mathrm{~mol} \%)$, and $\mathrm{CuI}(1.0 \mathrm{~mol} \%)$ in $\mathrm{NEt}_{3}(0.25$

M) was added by the phenylacetylene (1.2 equivalent). The resulting mixture was stirred under nitrogen atmosphere at $50^{\circ} \mathrm{C}$ overnight. After the reaction was finished, the mixture was filtered by short silica, then the solvent was evaporated under reduced pressure and the residue was purified by flash chromatography on silica gel to afford the desired product.

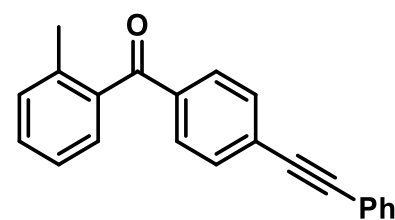

(4-(phenylethynyl)phenyl)(o-tolyl)methanone ${ }^{15}$ (9o) Purified by column

chromatography ( silica gel, PE:EA=10:1, v/v), Yellow solid, $781 \mathrm{mg}, 66 \%$ yield, ${ }^{1} \mathrm{H} \mathrm{NMR}\left(400 \mathrm{MHz}, \mathrm{CDCl}_{3}\right) \delta$ $7.75-7.66(\mathrm{~m}, 2 \mathrm{H}), 7.55-7.49(\mathrm{~m}, 2 \mathrm{H}), 7.49-7.43(\mathrm{~m}, 2 \mathrm{H}), 7.34-7.25(\mathrm{~m}, 4 \mathrm{H}), 7.25-7.14(\mathrm{~m}, 3 \mathrm{H}), 2.25(\mathrm{~s}$, $3 \mathrm{H}) .{ }^{13} \mathrm{C} \mathrm{NMR}\left(101 \mathrm{MHz}, \mathrm{CDCl}_{3}\right) \delta 197.8,138.4,136.9,136.8,131.8,131.6 .131 .1,130.4,130.1,128.9,128.5$, 128.5, 128.3, 125.3, 122.7, 92.9, 88.7, 20.1. 


\section{General procedure for iridium catalyzed hydrogenation.}

Procedure for solvent screening experiment: in an argon-filled glove box, a solution of $[\operatorname{Ir}(\mathrm{COD}) \mathrm{Cl}]_{2}(2.7 \mathrm{mg}$, $4 \mu \mathrm{mol})$ and Heng-PNP $(3.7 \mathrm{mg}, 8.4 \mu \mathrm{mol})$ in anhydrous dichloromethane $(0.8 \mathrm{~mL})$ was stirred at room temperature for $30 \mathrm{~min}$. A specified volume of the resulting solution $(50 \mu \mathrm{L}, 1 \mathrm{~mol} \%$ Ir catalyst) was transferred to a Score-Break ampule charged with substrate $(0.05 \mathrm{mmol}), 50 \mu \mathrm{L}$ of $\mathrm{KO}^{t} \mathrm{Bu}$ in ${ }^{i} \mathrm{PrOH}(0.1 \mathrm{M}, 10 \mathrm{~mol} \%)$ and $0.5 \mathrm{ml}$ of solvent. The ampule was placed into an autoclave, which was then pressurized with $50 \mathrm{~atm}$ of $\mathrm{H}_{2}$. The autoclave was stirred at room temperature for $24 \mathrm{~h}$. After releasing of $\mathrm{H}_{2}$, the mixture passed through a short silica gel plug, solvent was removed in vacuo. The crude product was analyzed by ${ }^{1} \mathrm{H}$ NMR to determine the conversion. The enantiomeric excess was determined by HPLC on a chiral stationary phase.

Table S1. Solvent screening for the asymmetric hydrogenation of $o$-bromobenzophenone

\begin{tabular}{cccccc} 
& & & \\
\hline & & & \\
\hline
\end{tabular}

${ }^{a}$ reaction conditions: ketone $(0.05 \mathrm{mmol})$, base $(5 \mathrm{mmol})$, ligand $(0.525 \mathrm{mmol}),[\operatorname{lr}(\mathrm{COD}) \mathrm{Cl}]_{2}$ $(0.025 \mathrm{mmol}), \mathrm{H}_{2}(50 \mathrm{~atm})$, solvent $(0.5 \mathrm{~mL}), 24 \mathrm{~h} .{ }^{b}$ Determined by integration of ${ }^{1} \mathrm{HNMR}$ spectra. ${ }^{c}$ Determined by integration of HPLC spectra. ${ }^{d}$ The solvent was degassed by bubbling.

General procedure for base screening and subtrate scope expansion experiment: in an argon-filled glove box, a solution of precatalyst $(3.9 \mathrm{mg}, 5 \mu \mathrm{mol})$ in toluene $(0.5 \mathrm{~mL})$ was prepared. A specified volume of the resulting 
solution $(50 \mu \mathrm{L}, 1 \mathrm{~mol} \%$ Ir catalyst) was transferred to a Score-Break ampule charged with substrate $(0.05 \mathrm{mmol})$, specified amount of base $(10 \mathrm{~mol} \%)$ and $0.5 \mathrm{~mL}$ of solvent. The ampule was placed into an autoclave, which was then charged with 50 atm of $\mathrm{H}_{2}$, the autoclave was stirred at room temperature for $48 \mathrm{~h}$. After releasing of $\mathrm{H}_{2}$, the mixture passed through a short silica gel plug, the solvent was removed in vacuo. The crude product was analyzed by ${ }^{1} \mathrm{H}$ NMR to determine the conversion. The enantiomeric excess was determined by HPLC on a chiral stationary phase.

Comparison of iridium precursors. There was no difference between the outcomes with $[\operatorname{Ir}(\mathrm{COD}) \mathrm{Cl}]_{2}$ and $\left[\operatorname{Ir}(\mathrm{COE})_{2} \mathrm{Cl}\right]_{2}$ as the precursor for hydrogenation reaction. However, we found that in situ formed iridium catalyst could not always yielded the best enantioselectivity we have achieved. Fortunately, AH with precatalyst gave reproducible results.

Table S2. Comparison of iridium precatalyst for biaryl ketone hydrogenation.

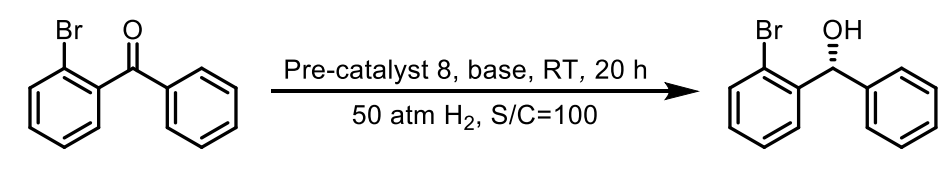

\begin{tabular}{ccccccc}
\hline Entry & Solvent & Catalyst & Base & Substrate/Base & Conv. $(\%)^{b}$ & ee $(\%)^{c}$ \\
\hline 1 & Hexane & In situ & ${ }^{t}$ BuOK & 10 & $>99$ & 92 \\
2 & Hexane & pre-catalyst & ${ }^{t}$ BuOK & 10 & $>99$ & 92 \\
\hline
\end{tabular}

${ }^{a}$ reaction conditions: ketone $(0.05 \mathrm{mmol}),{ }^{t} \mathrm{BuOK}(5 \mathrm{mmol})$, catalyst $(1 \mathrm{~mol} \%), \mathrm{H}_{2}(50 \mathrm{~atm})$, solvent $(0.5 \mathrm{~mL})$, $24 \mathrm{~h} .{ }^{b}$ Determined by integration of ${ }^{1} \mathrm{HNMR}$ spectra. ${ }^{c}$ Determined by integration of HPLC spectra. ${ }^{d}$ The solvent was degassed by freeze-pump-thaw.

Table S3. Base screening for the asymmetric hydrogenation of $o$-bromobenzophenone 


\begin{tabular}{|c|c|c|c|c|c|}
\hline Entry & Solvent & Base & Substrate/Base & Conv. $(\%)^{b}$ & ee $(\%)^{c}$ \\
\hline 1 & Pentane & ${ }^{t} \mathrm{BuOK}$ & 10 & $>99$ & 92 \\
\hline 2 & Hexane & ${ }^{t} \mathrm{BuOK}$ & 10 & $>99$ & 92 \\
\hline 3 & Hexane & $\mathrm{K}_{2} \mathrm{CO}_{3}$ & 10 & 83 & 96 \\
\hline 4 & Hexane & $\mathrm{KOH}$ & 10 & $>99$ & 96 \\
\hline 5 & Hexane & ${ }^{t} \mathrm{BuONa}$ & 10 & $>99$ & 81 \\
\hline 6 & Hexane & ${ }^{t} \mathrm{BuOLi}$ & 10 & $>99$ & 97.2 \\
\hline 7 & Hexane & $\mathrm{LiOH}$ & 10 & $>99$ & 96 \\
\hline 8 & Hexane & $\mathrm{Cs}_{2} \mathrm{CO}_{3}$ & 10 & $>99$ & 97 \\
\hline
\end{tabular}

${ }^{a}$ reaction conditions: ketone $(0.05 \mathrm{mmol})$, base $(5 \mu \mathrm{mol})$, precatalyst $8(0.5 \mu \mathrm{mol}), \mathrm{H}_{2}(50 \mathrm{~atm})$, solvent $(0.5$

$\mathrm{mL}), 24 \mathrm{~h} .{ }^{b}$ Determined by integration of ${ }^{1} \mathrm{H}$ NMR spectra. ${ }^{c}$ Determined by

integration of HPLC spectra. ${ }^{d}$ The solvent was degassed by freeze-pump-thaw.

\section{Comparison of Heng-PNP and f-amphox in AH of diaryl ketones}

Compared to [Ir/Heng-PNP], [Ir/f-amphox] only gave hydrogenation result with moderate to poor ee values under optimized reaction conditions (Table S4).

Table S4. Enantioselectivity comparison between [Ir-L] and [Ir-(f-Amphox)]

\begin{tabular}{|c|c|c|c|c|}
\hline$[\mathrm{Ir}-\mathrm{L}]$ & $90 \%$ ee & $93 \%$ ee & $93 \%$ ee & $90 \%$ ee \\
\hline [Ir-(f-Amphox)] & $62 \%$ ee & $55 \%$ ee & $64 \%$ ee & Racemic \\
\hline
\end{tabular}

\section{Measurement of turnover number (TON).}

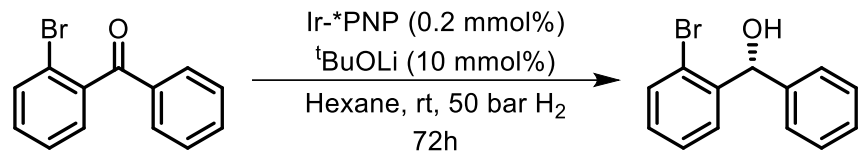

In an argon-filled glove box, to a $10-\mathrm{mL}$ vial equipped with a magnetic stirring bar was added $25 \mu \mathrm{L}$ catalyst solution Ir/Heng-PNP(20 $\mu \mathrm{mol} / \mathrm{mL}$ in toluene), ${ }^{\mathrm{t}} \mathrm{BuOLi}(0.025 \mathrm{mmol}, 2 \mathrm{mg})$ and $0.25 \mathrm{~mL}$ hexane. Then the mixture 
was transferred to a stain-less autoclave and then was purged by three cycles $\mathrm{H}_{2}(10 \mathrm{~atm})$ then pressurized with $\mathrm{H}_{2}$ (50 atm). Then the autoclave was stirred at room temperature. After 72 hours, the hydrogen gas was released slowly in a hood. The reaction mixture was diluted with DCM and filtered via a short silica gel plug. 98\% yield and 95\% ee of (R)-(2-bromophenyl)(phenyl)methanol was obtained (TON: 500). 


\section{Characterization data of the hydrogenation products.}

(R)-(2-bromophenyl)(phenyl)methanol ${ }^{19}$ (10a) Purified with flash chromatography on silica (PE:EA=8:1), Yellow oil, $12.5 \mathrm{mg}, 96 \%$ yield, 96\% ee, $[\alpha]_{\mathrm{D}^{24}}=+35.4\left(\mathrm{c}=0.39, \mathrm{CHCl}_{3}\right)$; The enantiomeric excess was determined by UPLC on Chiralpak IB-U column, hexane:

isopropanol $=95: 5$; flow rate $=0.5 \mathrm{~mL} / \mathrm{min}$; $\mathrm{UV}$ detection at $210 \mathrm{~nm}$; $\mathrm{tR}=3.82 \mathrm{~min}$ (major), $4.42 \mathrm{~min}$ (minor). ${ }^{1} \mathrm{H}$ $\operatorname{NMR}\left(400 \mathrm{MHz}, \mathrm{CDCl}_{3}\right) \delta 7.48(\mathrm{dd}, J=7.8 \mathrm{~Hz}, 1.8 \mathrm{~Hz}, 1 \mathrm{H}), 7.44(\mathrm{dd}, J=8.0 \mathrm{~Hz}, 1.3 \mathrm{~Hz}, 1 \mathrm{H}), 7.27-7.14(\mathrm{~m}, 4 \mathrm{H})$, $7.33-7.28(\mathrm{~m}, 2 \mathrm{H}), 7.05(\mathrm{td}, J=7.7 \mathrm{~Hz}, 1.8 \mathrm{~Hz}, 1 \mathrm{H}), 6.08(\mathrm{~s}, 1 \mathrm{H}), 2.49(\mathrm{~s}, 1 \mathrm{H}) \cdot{ }^{13} \mathrm{C}$ NMR $\left(101 \mathrm{MHz}, \mathrm{CDCl}_{3}\right) \delta$ $142.5,142.2,132.9,129.2,128.5,127.8,127.8,127.1,122.8,77.4,77.1,76.8,74.8$.

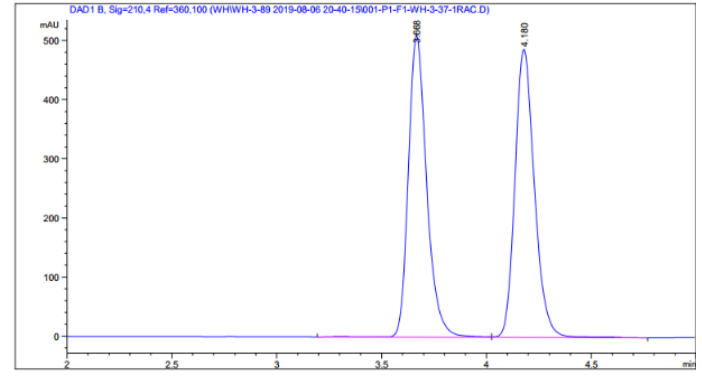

Signal 1: DAD1 B, Sig=210,4 Ref=360,100

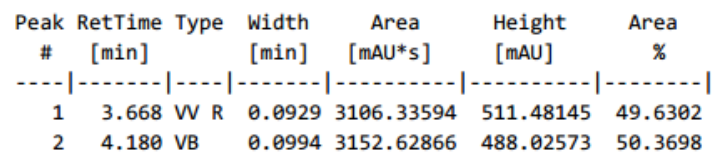

Totals :

$6258.96460 \quad 999.50717$

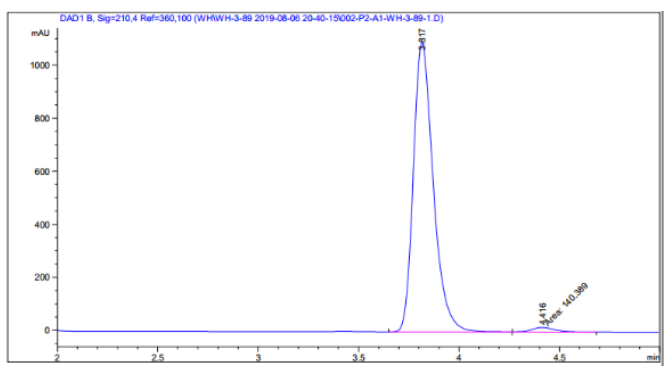

Signal 1: DAD1 B, Sig=210,4 Ref=360,100

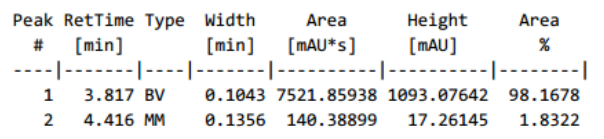

Totals :

7662.248371110 .33787 
<smiles>O[C@H](c1ccccc1)c1ccccc1Cl</smiles>

$(R)$-(2-chlorophenyl)(phenyl)methanol ${ }^{19}$ (10b) Purified with flash chromatography on silica (PE:EA=8:1), Colorless oil, $10.4 \mathrm{mg}, 95 \%$ yield, 95\% ee, $[\alpha]_{\mathrm{D}}{ }^{24}=+29.7\left(\mathrm{c}=0.62, \mathrm{CHCl}_{3}\right)$; The enantiomeric excess was determined by UPLC on Chiralpak IB-U column, hexane: isopropanol $=95: 5$; flow rate $=0.5 \mathrm{~mL} / \mathrm{min}$; $\mathrm{UV}$ detection at $210 \mathrm{~nm}$; $\mathrm{tR}=3.55 \mathrm{~min}$ (major), $3.89 \mathrm{~min}$ (minor). ${ }^{1} \mathrm{H} \mathrm{NMR}\left(400 \mathrm{MHz}, \mathrm{CDCl}_{3}\right) \delta 7.61(\mathrm{dd}, J=7.7$, $1.8 \mathrm{~Hz}, 1 \mathrm{H}), 7.41-7.38(\mathrm{~m}, 2 \mathrm{H}), 7.36-7.26(\mathrm{~m}, 5 \mathrm{H}), 7.25-7.20(\mathrm{~m}, 1 \mathrm{H}), 6.23(\mathrm{~s}, 1 \mathrm{H}) .{ }^{13} \mathrm{C}$ NMR $(101 \mathrm{MHz}$, $\left.\mathrm{CDCl}_{3}\right) \delta 142.3,141.0,129.6,128.8,128.5,128.1,127.8,127.1,126.9,77.4,77.0,76.7,72.7$.

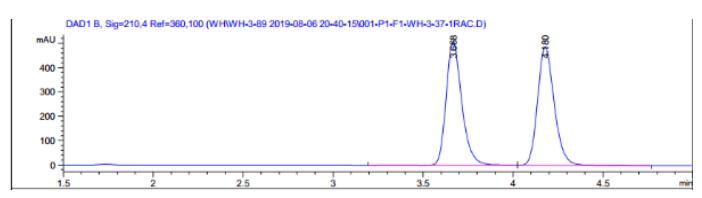

Signal 2: DAD1 B, Sig $=210,4$ Ref $=360,100$

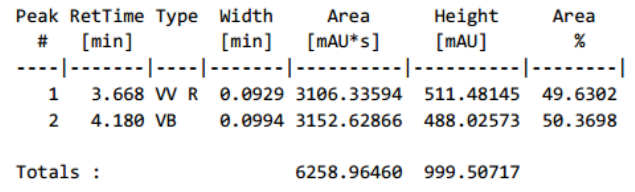
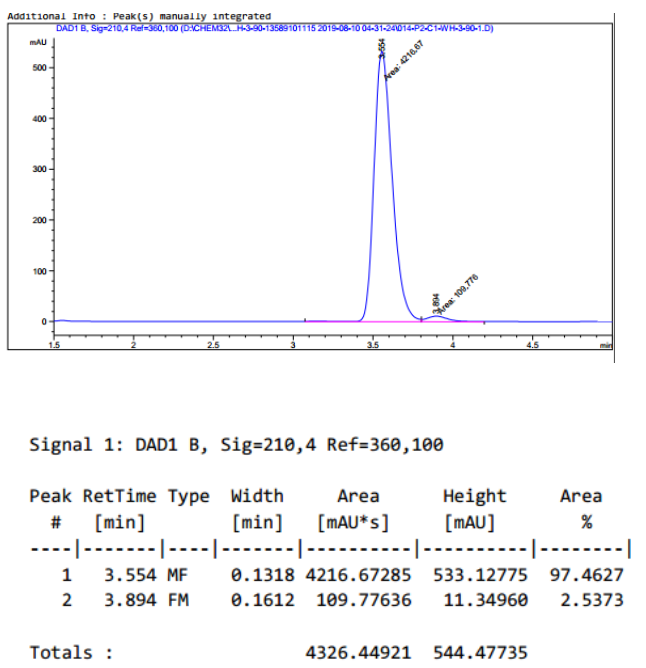
<smiles>O[C@@H](c1ccccc1)c1ccccc1I</smiles>

(R)-(2-iodophenyl)(phenyl)methanol ${ }^{20}$ (10c) Purified with flash chromatography on silica (PE:EA=8:1), White solid, $14.0 \mathrm{mg}, 90 \%$ yield, 97\% ee, $[\alpha]_{\mathrm{D}}{ }^{24}=+37.1\left(\mathrm{c}=0.21, \mathrm{CHCl}_{3}\right)$; The enantiomeric excess was determined by UPLC on Chiralpak IB-U column, hexane:isopropanol $=95: 5$; flow rate $=0.5 \mathrm{~mL} / \mathrm{min}$; UV detection at $210 \mathrm{~nm}$; $\mathrm{tR}=3.88 \mathrm{~min}$ (major), $4.51 \mathrm{~min}$ (minor). ${ }^{1} \mathrm{H} \mathrm{NMR}\left(600 \mathrm{MHz}, \mathrm{CDCl}_{3}\right) \delta 7.83$ (dd, $J=8.0,1.2$ $\mathrm{Hz}, 1 \mathrm{H}), 7.53(\mathrm{dd}, J=7.8,1.7 \mathrm{~Hz}, 1 \mathrm{H}), 7.44-7.40(\mathrm{~m}, 2 \mathrm{H}), 7.37(\mathrm{td}, J=7.5,1.2 \mathrm{~Hz}, 1 \mathrm{H}), 7.36-7.32(\mathrm{~m}, 2 \mathrm{H}), 7.30$ $-7.27(\mathrm{~m}, 1 \mathrm{H}), 6.99(\mathrm{td}, J=7.6,1.8 \mathrm{~Hz}, 1 \mathrm{H}), 6.07(\mathrm{~d}, J=3.3 \mathrm{~Hz}, 1 \mathrm{H}), 2.37(\mathrm{~d}, J=4.1 \mathrm{~Hz}, 1 \mathrm{H}) .{ }^{13} \mathrm{C} \mathrm{NMR}(151 \mathrm{MHz}$, $\left.\mathrm{CDCl}_{3}\right) \delta 144.4,141.1,138.6,128.4,127.6,127.5,127.4,126.8,126.2,97.6,78.0,76.2,76.0,75.8$.

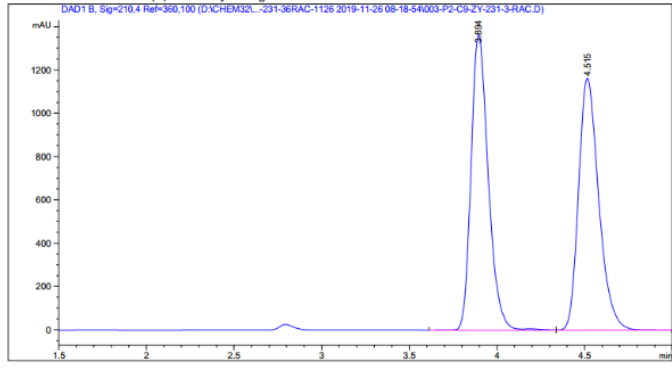

Signal 1: DAD1 B, Sig=210,4 Ref=360,100

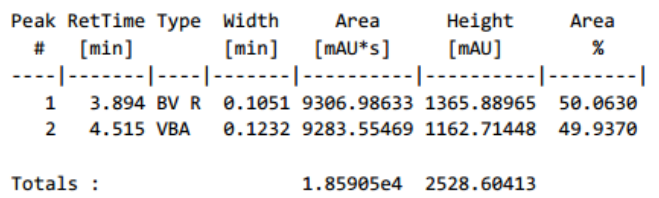

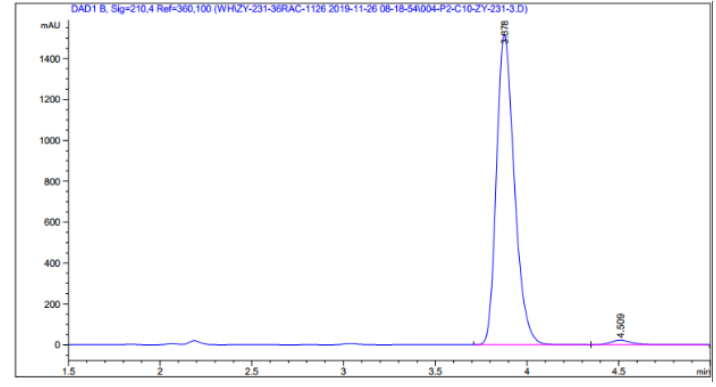

SIgna1 1: UAU1 $\mathrm{B}, \mathrm{SIg}=\angle 10,4$ ket=s60,100

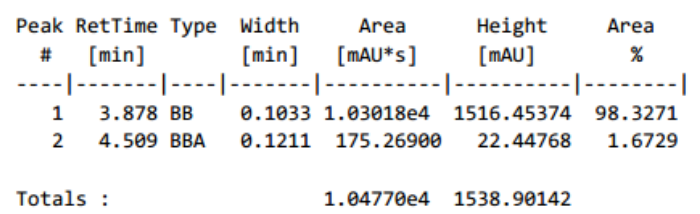


(R)-phenyl(o-tolyl)methanol ${ }^{19}$ (10d) Purified with flash chromatography on silica (PE:EA=8:1), White solid, $8.4 \mathrm{mg}, 85 \%$ yield, $96 \% e e,[\alpha]_{\mathrm{D}}{ }^{24}=-5.63\left(\mathrm{c}=0.15, \mathrm{CHCl}_{3}\right)$; The enantiomeric excess was determined by UPLC on Chiralpak IC-U column, hexane:isopropanol $=98: 2$; flow rate $=0.5 \mathrm{~mL} / \mathrm{min}$; UV detection at $210 \mathrm{~nm}$; $\mathrm{tR}=5.11 \mathrm{~min}$ (major), $4.57 \mathrm{~min}$ (minor). ${ }^{1} \mathrm{H} \mathrm{NMR}\left(400 \mathrm{MHz}, \mathrm{CDCl}_{3}\right) \delta 7.45$ (dd, $\left.J=7.5,1.6 \mathrm{~Hz}, 1 \mathrm{H}\right), 7.25(\mathrm{~d}, J=$ $4.4 \mathrm{~Hz}, 4 \mathrm{H}), 7.22-7.17(\mathrm{~m}, 2 \mathrm{H}), 7.17-7.11(\mathrm{~m}, 1 \mathrm{H}), 7.07$ (dd, J = 7.4, 1.6 Hz, 1H), $5.95-5.92(\mathrm{~m}, 1 \mathrm{H}), 2.18(\mathrm{~s}$, $3 \mathrm{H}), 2.09(\mathrm{~d}, J=3.6 \mathrm{~Hz}, 1 \mathrm{H}) .{ }^{3} \mathrm{C} \mathrm{NMR}\left(101 \mathrm{MHz}, \mathrm{CDCl}_{3}\right) \delta 142.8,132.4,129.5,128.4,127.8,127.6,126.5,125.6$, $125.5,77.3,77.2,77.0,76.7,70.9$.

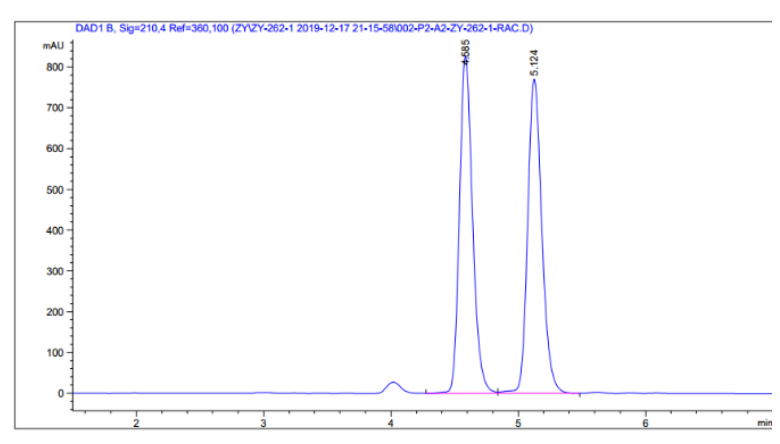

Signal 1: DAD1 B, Sig=210,4 $\operatorname{Ref}=360,100$

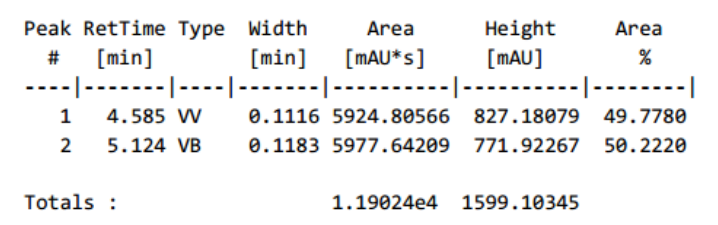

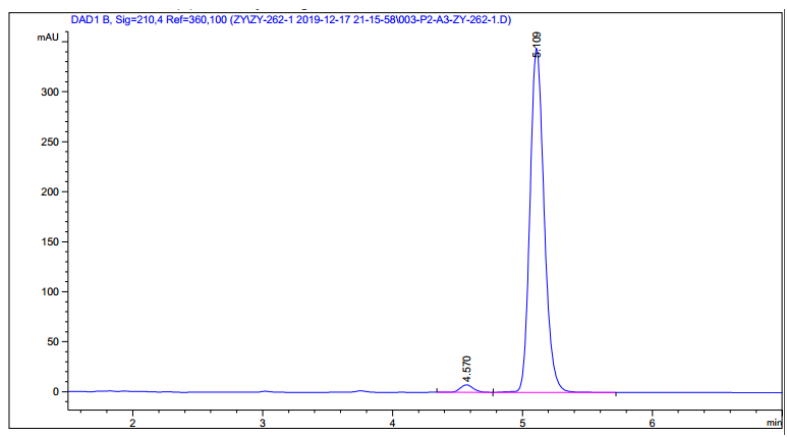

Signal 1: DAD1 B, Sig=210,4 Ref $=360,100$

\begin{tabular}{|c|c|c|c|c|c|}
\hline $\begin{array}{c}\text { Peak } \\
\#\end{array}$ & $\begin{array}{l}\text { RetTime Type } \\
\text { [min] }\end{array}$ & $\begin{array}{l}\text { Width } \\
\text { [min] }\end{array}$ & $\begin{array}{c}\text { Area } \\
\text { [mAU*s] }\end{array}$ & $\begin{array}{l}\text { Height } \\
\text { [mAU] }\end{array}$ & $\begin{array}{c}\text { Area } \\
\%\end{array}$ \\
\hline$\cdots$ & $|\cdots-\ldots|-\ldots$ & (n) & | & | & | \\
\hline 1 & $4.570 \mathrm{BB}$ & 0.1070 & 52.31040 & 7.53191 & 1.9645 \\
\hline 2 & 5.109 BВ & 0.1165 & 2610.52466 & 344.28690 & 98.0355 \\
\hline Tota & & & 2662.83506 & 351.81880 & \\
\hline
\end{tabular}


(R)-phenyl(2-(trifluoromethyl)phenyl)methanol ${ }^{19}(10 \mathrm{e})$ Purified with flash chromatography on silica (PE:EA=8:1), Colorless oil, $7.1 \mathrm{mg}, 73 \%$ yield, 94\% ee. $[\alpha]_{\mathrm{D}}{ }^{24}=+32.8\left(\mathrm{c}=0.56, \mathrm{CHCl}_{3}\right)$; The enantiomeric excess was determined by UPLC on Chiralpak IB-U column, hexane:isopropanol $=98: 2$; flow rate $=0.5 \mathrm{~mL} / \mathrm{min}$; UV detection at $210 \mathrm{~nm}$; $\mathrm{tR}=3.71 \mathrm{~min}$ (major), $4.52 \mathrm{~min}$ (minor). ${ }^{1} \mathrm{H} \mathrm{NMR}\left(400 \mathrm{MHz}, \mathrm{CDCl}_{3}\right) \delta 7.60-7.54(\mathrm{~m}, 2 \mathrm{H})$, $7.46(\mathrm{td}, J=7.7,1.3 \mathrm{~Hz}, 1 \mathrm{H}), 7.33-7.22(\mathrm{~m}, 5 \mathrm{H}), 7.22-7.16(\mathrm{~m}, 1 \mathrm{H}), 6.23(\mathrm{~d}, J=1.9 \mathrm{~Hz}, 1 \mathrm{H}), 2.33(\mathrm{~d}, J=3.4 \mathrm{~Hz}$, 1H). ${ }^{13} \mathrm{C}$ NMR $\left(101 \mathrm{MHz}, \mathrm{CDCl}_{3}\right) \delta 141.7,141.3,131.3,128.5,127.4,126.7,126.5,126.4,125.4,124.7,124.5$ (q, $J$ $=5.5 \mathrm{~Hz}), 122.0,76.3,76.0,75.7,69.8(\mathrm{q}, J=25.3 \mathrm{~Hz}) .{ }^{19} \mathrm{~F} \mathrm{NMR}\left(376 \mathrm{MHz}, \mathrm{CDCl}_{3}\right) \delta$-57.6.

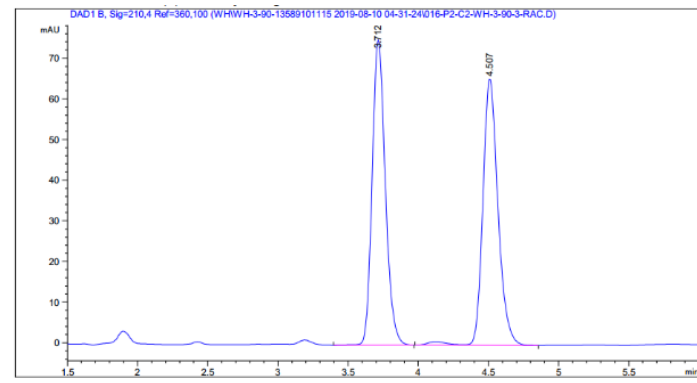

Signal 1: DAD1 B, Sig $=210,4$ Ref $=360,100$

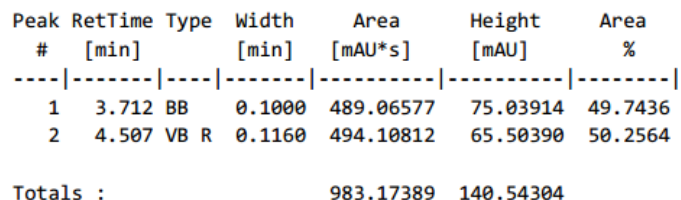

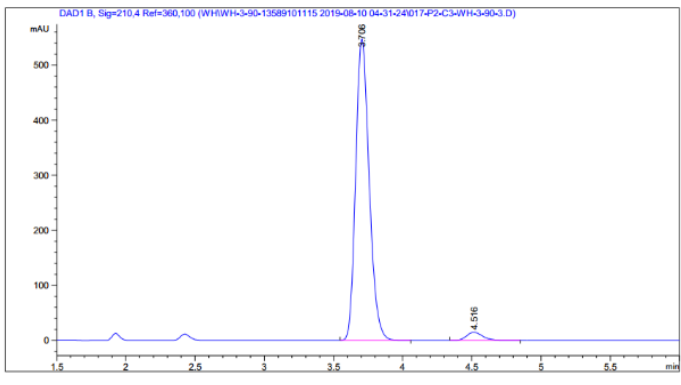

Signal 1: DAD1 B, Sig=210,4 Ref=360,100

\begin{tabular}{|c|c|c|c|c|c|c|}
\hline $\begin{array}{c}\text { Peak } \\
\#\end{array}$ & $\begin{array}{c}\text { RetTime } \\
\text { [min] }\end{array}$ & Type & $\begin{array}{l}\text { Width } \\
\text { [min] }\end{array}$ & $\begin{array}{c}\text { Area } \\
{\left[\mathrm{mAU}{ }^{*} \mathrm{~s}\right]}\end{array}$ & $\begin{array}{l}\text { Height } \\
{[\mathrm{mAU}]}\end{array}$ & $\begin{array}{c}\text { Area } \\
\%\end{array}$ \\
\hline$\cdots-1$ & 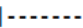 & & & $\cdots$ & & $\cdots$ \\
\hline 1 & 3.706 & BB & 0.1051 & 3708.56592 & 547.25793 & 96.9624 \\
\hline 2 & 4.516 & BB & 0.1219 & 116.17924 & 14.74297 & 3.0376 \\
\hline Tota & & & & 3824.74516 & 562.00090 & \\
\hline
\end{tabular}




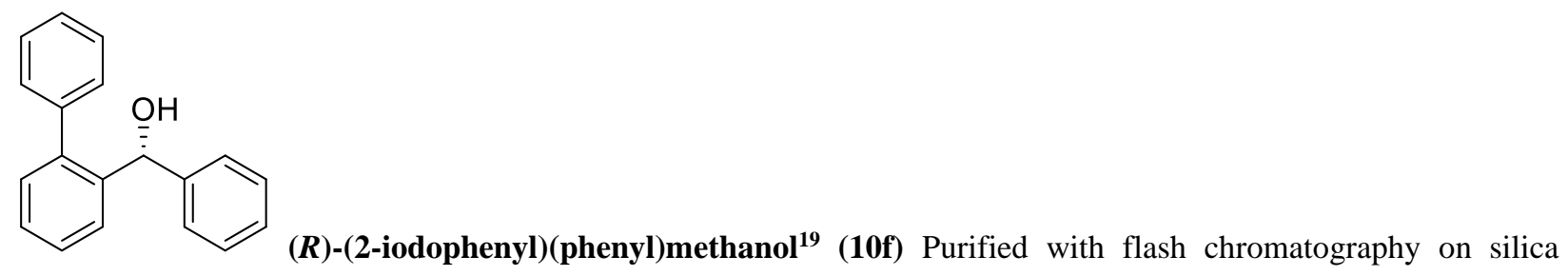
(PE:EA=8:1), White solid, $8.2 \mathrm{mg}, 63 \%$ yield, 94\% ee. $[\alpha]_{\mathrm{D}}{ }^{24}=+37.1\left(\mathrm{c}=0.21, \mathrm{CHCl}_{3}\right)$; The enantiomeric excess was determined by UPLC on Chiralpak IC-U column, hexane:isopropanol = 97:3; flow rate $=0.5 \mathrm{~mL} / \mathrm{min}$; UV detection at $210 \mathrm{~nm} ; \mathrm{tR}=2.37 \mathrm{~min}$ (major), $2.53 \mathrm{~min}$ (minor). ${ }^{1} \mathrm{H} \mathrm{NMR}\left(400 \mathrm{MHz}, \mathrm{CDCl}_{3}\right) \delta 7.46(\mathrm{dd}, J=7.7,1.5$ $\mathrm{Hz}, 1 \mathrm{H}), 7.31-7.26(\mathrm{~m}, 4 \mathrm{H}), 7.22(\mathrm{dd}, J=7.4,1.5 \mathrm{~Hz}, 1 \mathrm{H}), 7.20-7.10(\mathrm{~m}, 6 \mathrm{H}), 7.10-7.04(\mathrm{~m}, 2 \mathrm{H}), 5.83(\mathrm{~s}, 1 \mathrm{H})$, 2.12 (s, 1H). ${ }^{13} \mathrm{C}$ NMR $\left(101 \mathrm{MHz}, \mathrm{CDCl}_{3}\right) \delta 142.8,140.3,140.0,139.8,129.0,128.3,127.2,127.1,126.8,126.4$, 126.2, 125.6, 76.3, 76.0, 75.7, 71.3.
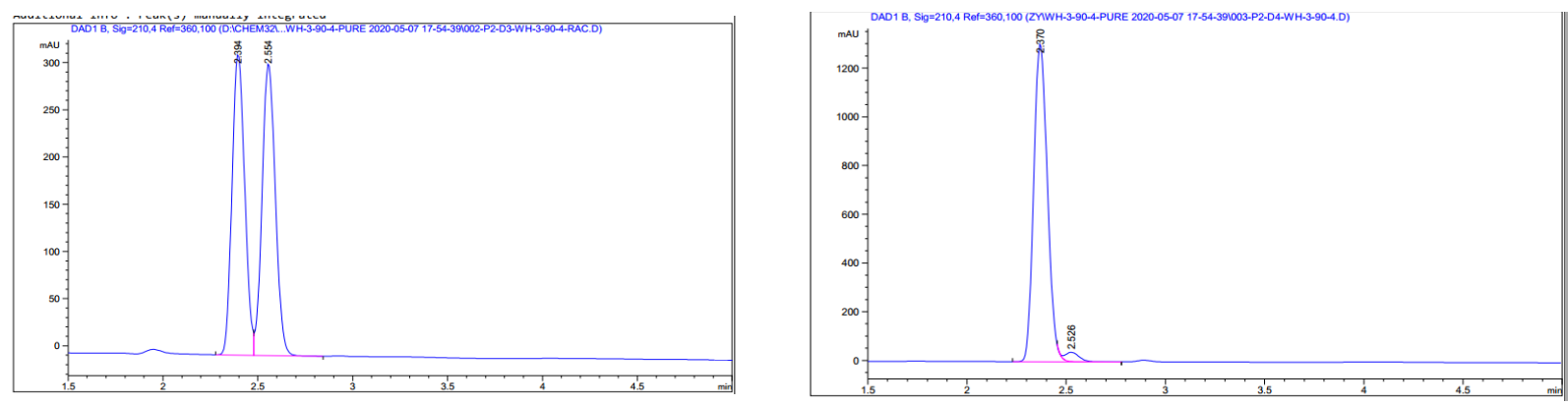

Signal 1: DAD1 B, Sig $=210,4$ Ref $=360,100$

Signal 1: DAD1 B, Sig $=210,4$ Ref $=360,100$

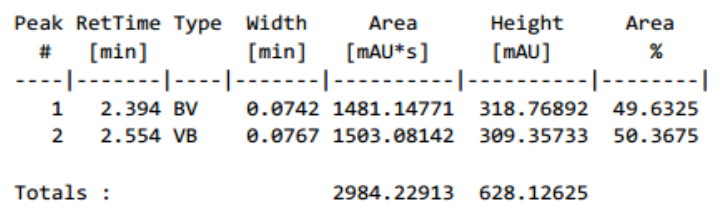


(R)-naphthalen-1-yl(phenyl)methanol ${ }^{31}(\mathbf{1 0 g})$ Purified with flash chromatography on silica (PE:EA=8:1), Colorless oil, $12.4 \mathrm{mg}, 92 \%$ yield, $96 \% e e .[\alpha]_{\mathrm{D}}{ }^{24}=+32.6\left(\mathrm{c}=0.50, \mathrm{CHCl}_{3}\right)$; The enantiomeric excess was determined by UPLC on Chiralpak IC-U column, hexane:isopropanol $=97: 3$; flow rate $=0.5 \mathrm{~mL} / \mathrm{min}$; UV detection at $210 \mathrm{~nm}$; $\mathrm{tR}=6.63 \mathrm{~min}$ (major), $5.16 \mathrm{~min}$ (minor). ${ }^{1} \mathrm{H} \mathrm{NMR}\left(400 \mathrm{MHz}, \mathrm{CDCl}_{3}\right) \delta 8.05-8.01(\mathrm{~m}, 1 \mathrm{H})$, $7.88-7.84(\mathrm{~m}, 1 \mathrm{H}), 7.81(\mathrm{~d}, J=8.2 \mathrm{~Hz}, 1 \mathrm{H}), 7.63(\mathrm{~d}, J=7.1 \mathrm{~Hz}, 1 \mathrm{H}), 7.51-7.39(\mathrm{~m}, 5 \mathrm{H}), 7.35-7.30(\mathrm{~m}, 2 \mathrm{H}), 7.30$ $-7.23(\mathrm{~m}, 1 \mathrm{H}), 6.53(\mathrm{~d}, J=2.9 \mathrm{~Hz}, 1 \mathrm{H}), 2.37(\mathrm{~d}, J=3.8 \mathrm{~Hz}, 1 \mathrm{H}) .{ }^{13} \mathrm{C} \mathrm{NMR}\left(101 \mathrm{MHz}, \mathrm{CDCl}_{3}\right) \delta 143.1,138.8,134.0$, $130.7,128.8,128.6,128.5,127.7,127.1,126.2,125.6,125.4,124.6,124.0,73.7$. .

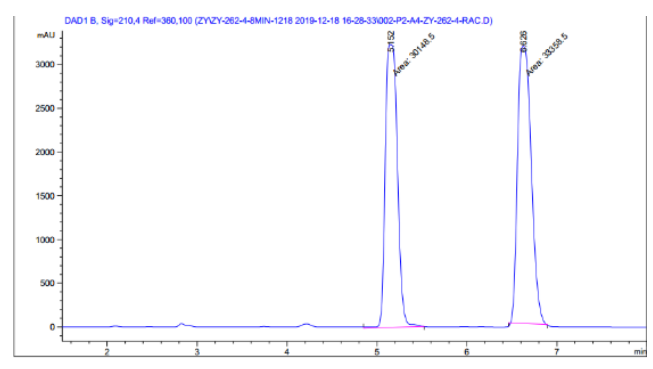

Signal 1: DAD1 B, Sig=210,4 Ref=360,100

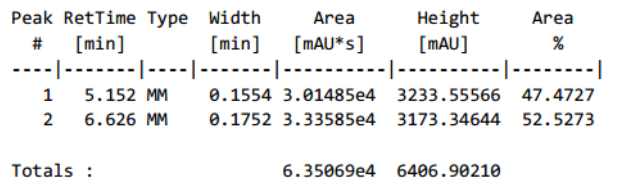

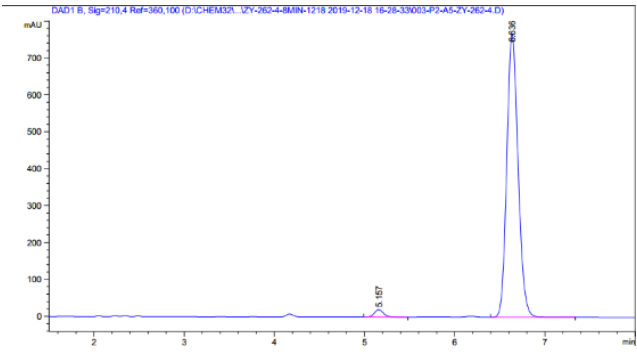

Signal 1: DAD1 B, Sig=210,4 Ref=360,100

\begin{tabular}{|c|c|c|c|c|c|c|}
\hline $\begin{array}{c}\text { Peak } \\
\#\end{array}$ & $\begin{array}{c}\text { RetTime } \\
\text { [min] }\end{array}$ & Type & $\begin{array}{l}\text { Width } \\
\text { [min] }\end{array}$ & $\begin{array}{c}\text { Area } \\
{\left[\mathrm{mAU}^{*} \mathrm{~s}\right]}\end{array}$ & $\begin{array}{l}\text { Height } \\
{[\mathrm{mAU}]}\end{array}$ & $\begin{array}{c}\text { Area } \\
\%\end{array}$ \\
\hline$\cdots-1$ & . & & 等 & - & - & | \\
\hline 1 & 5.157 & BB & 0.1068 & 137.97455 & 19.92892 & 2.0322 \\
\hline 2 & 6.636 & BB & 0.1353 & 6651.52783 & 766.02435 & 97.9678 \\
\hline Tota. & & & & 6789.50238 & 785.95328 & \\
\hline
\end{tabular}


<smiles>O[C@@H](c1ccoc1)c1ccccc1Br</smiles>

(R)-naphthalen-1-yl(phenyl)methanol ${ }^{32}$ (10h) Purified with flash chromatography on silica (PE:EA=8:1), Colorless oil, $11.4 \mathrm{mg}, 90 \%$ yield, $91 \% e e .[\alpha]_{\mathrm{D}}{ }^{24}=+37.1\left(\mathrm{c}=0.21, \mathrm{CHCl}_{3}\right)$; The enantiomeric excess was determined by UPLC on Chiralpak IB-U column, hexane:isopropanol $=95: 5$; flow rate $=0.5 \mathrm{~mL} / \mathrm{min}$; UV detection at $210 \mathrm{~nm}$; $\mathrm{tR}=3.29 \min$ (major), $3.87 \mathrm{~min}$ (minor). ${ }^{1} \mathrm{H} \mathrm{NMR}\left(400 \mathrm{MHz}, \mathrm{CDCl}_{3}\right) \delta 7.63$ (dd, $J=7.8,1.7$ $\mathrm{Hz}, 1 \mathrm{H}), 7.54(\mathrm{dd}, J=8.0,1.2 \mathrm{~Hz}, 1 \mathrm{H}), 7.40-7.30(\mathrm{~m}, 3 \mathrm{H}), 7.17(\mathrm{td}, J=7.7,1.8 \mathrm{~Hz}, 1 \mathrm{H}), 6.37(\mathrm{~d}, J=1.8 \mathrm{~Hz}, 1 \mathrm{H})$, $6.14(\mathrm{~d}, J=2.8 \mathrm{~Hz}, 1 \mathrm{H}), 2.33(\mathrm{~d}, J=4.0 \mathrm{~Hz}, 1 \mathrm{H}) .{ }^{13} \mathrm{C} \mathrm{NMR}\left(101 \mathrm{MHz}, \mathrm{CDCl}_{3}\right) \delta 143.4,142.0,140.3,132.8,129.3$, 128.0, 127.8, 127.3, 122.3, 109.2, 68.4.

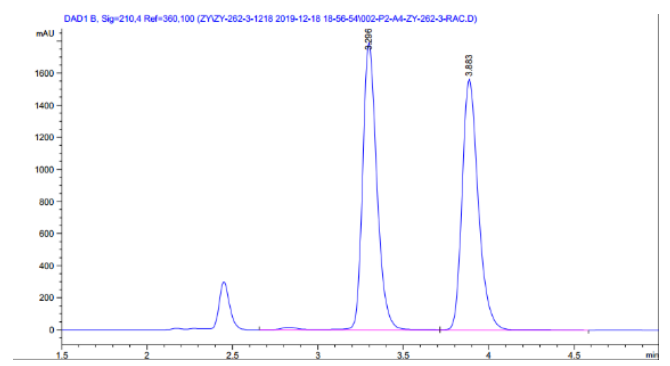

Signal 1: DAD1 B, Sig=210,4 Ref $=360,100$

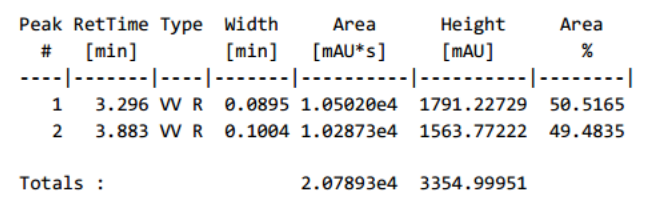

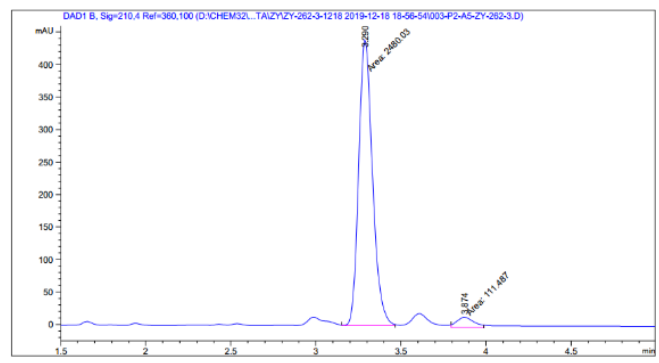

Signal 1: DAD1 B, Sig=210,4 Ref $=360,100$

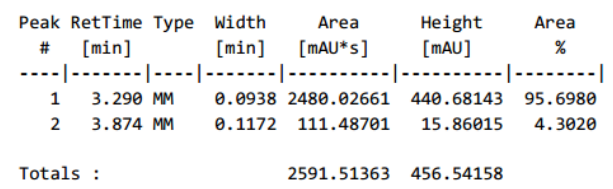


OMe (R)-(2-bromophenyl)(4-methoxyphenyl)methanol ${ }^{28}$ (10i) Purified with flash chromatography on silica (PE:EA=8:1), Colorless oil, $6.7 \mathrm{mg}, 46 \%$ yield, $96 \% e e .[\alpha]_{\mathrm{D}}^{24}=+14.2\left(\mathrm{c}=0.61, \mathrm{CHCl}_{3}\right)$; The enantiomeric excess was determined by UPLC on Chiralpak IC-U column, hexane:isopropanol = 95:5; flow rate $=0.5 \mathrm{~mL} / \mathrm{min}$; $\mathrm{UV}$ detection at $210 \mathrm{~nm} ; \mathrm{tR}=5.21 \mathrm{~min}$ (major), $5.56 \mathrm{~min}$ (minor). ${ }^{1} \mathrm{H} \mathrm{NMR}\left(400 \mathrm{MHz}, \mathrm{CDCl}_{3}\right) \delta 7.59$ (dd, $J=7.8,1.7 \mathrm{~Hz}, 1 \mathrm{H}), 7.49$ (dd, $J=8.0,1.3 \mathrm{~Hz}, 1 \mathrm{H}), 7.31(\mathrm{td}, J=7.6,1.3 \mathrm{~Hz}, 1 \mathrm{H}), 7.27-7.22(\mathrm{~m}, 2 \mathrm{H}), 7.10(\mathrm{td}$, $J=7.7,1.8 \mathrm{~Hz}, 1 \mathrm{H}), 6.84-6.79(\mathrm{~m}, 2 \mathrm{H}), 6.05(\mathrm{~d}, J=2.5 \mathrm{~Hz}, 1 \mathrm{H}), 3.74(\mathrm{~s}, 3 \mathrm{H}), 2.71(\mathrm{~d}, J=3.1 \mathrm{~Hz}, 1 \mathrm{H}) .{ }^{13} \mathrm{C} \mathrm{NMR}$ $\left(101 \mathrm{MHz}, \mathrm{CDCl}_{3}\right) \delta 159.1,142.8,134.5,132.8,129.0,128.6,128.2,127.7,122.7,113.9,74.4,55.3$.
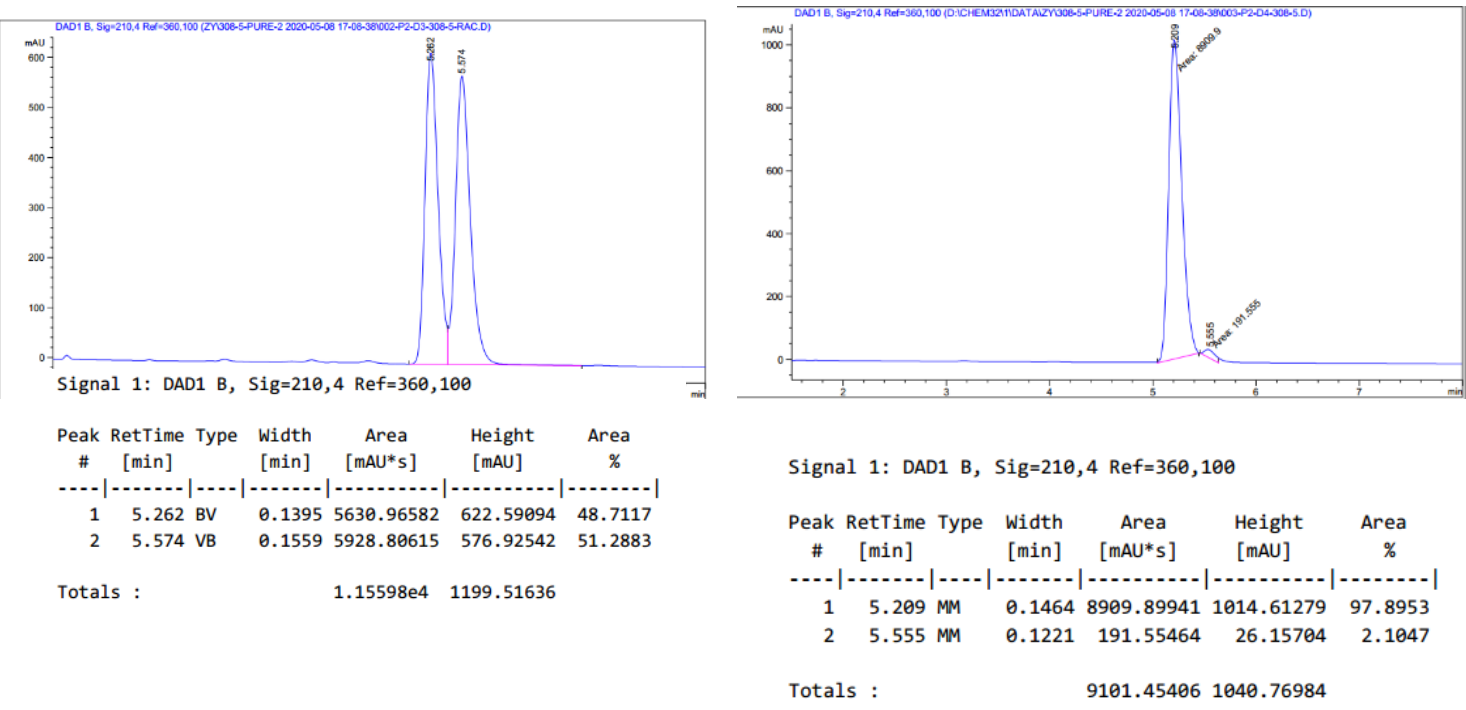
(R)-(2-bromophenyl)(p-tolyl)methanol ${ }^{28}(\mathbf{1 0 j})$ Purified with flash chromatography on silica (PE:EA=8:1), Colorless oil, $11.9 \mathrm{mg}, 86 \%$ yield, $95 \% e e .[\alpha]_{\mathrm{D}}^{24}=+31.4\left(\mathrm{c}=0.59, \mathrm{CHCl}_{3}\right)$; The enantiomeric excess was determined by UPLC on Chiralpak OD-H column, hexane:isopropanol $=90: 10$; flow rate $=0.8 \mathrm{~mL} / \mathrm{min}$; UV detection at $210 \mathrm{~nm}$; $\mathrm{tR}=9.83 \mathrm{~min}$ (major), $12.91 \mathrm{~min}$ (minor). ${ }^{1} \mathrm{H} \mathrm{NMR}\left(600 \mathrm{MHz}, \mathrm{CDCl}_{3}\right) \delta 7.54$ (dd, $J=7.8,1.7$ $\mathrm{Hz}, 1 \mathrm{H}), 7.46(\mathrm{dd}, J=8.0,1.2 \mathrm{~Hz}, 1 \mathrm{H}), 7.26(\mathrm{~d}, J=1.2 \mathrm{~Hz}, 1 \mathrm{H}), 7.19(\mathrm{~d}, J=8.1 \mathrm{~Hz}, 2 \mathrm{H}), 7.07(\mathrm{dd}, J=9.0,2.0 \mathrm{~Hz}$, 3H), $6.03-5.99(\mathrm{~m}, 1 \mathrm{H}), 2.90(\mathrm{~d}, J=3.3 \mathrm{~Hz}, 1 \mathrm{H}), 2.28(\mathrm{~s}, 3 \mathrm{H}) .{ }^{13} \mathrm{C} \mathrm{NMR}\left(151 \mathrm{MHz}, \mathrm{CDCl}_{3}\right) \delta 142.8,139.4,137.5$, $132.8,129.2,129.0,128.9,128.4,127.7,127.2,122.8,77.4,77.2,77.0,74.6,21.3$.
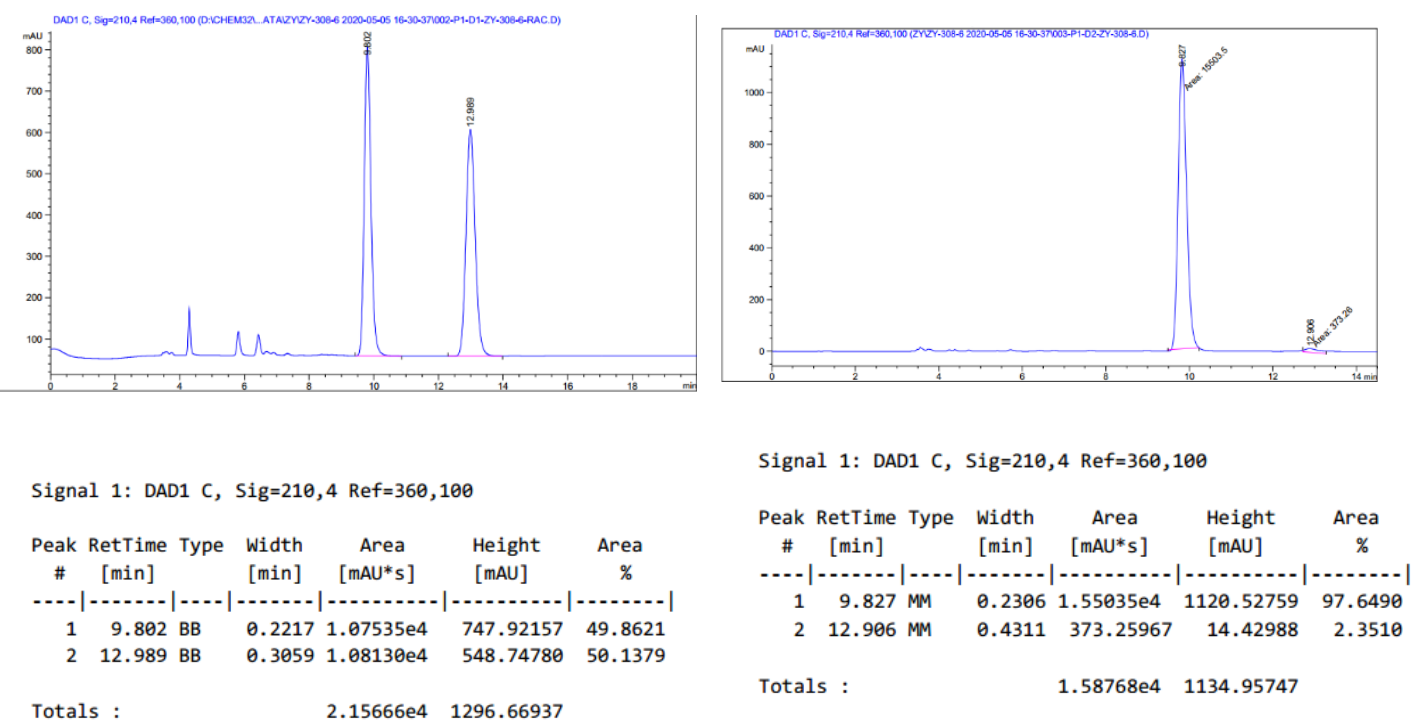
<smiles>O[C@H](c1ccc(Cl)cc1)c1ccccc1Br</smiles>

(R)-(2-bromophenyl)(4-chlorophenyl)methanol ${ }^{28} \quad(10 k)$ Purified with flash chromatography on silica (PE:EA=8:1), Colorless oil, $12.8 \mathrm{mg}, 96 \%$ yield, $97 \% e e .[\alpha]_{\mathrm{D}}^{24}=+53.8\left(\mathrm{c}=0.72, \mathrm{CHCl}_{3}\right)$; The enantiomeric excess was determined by UPLC on Chiralpak OD-H column, hexane:isopropanol = 90:10; flow rate $=0.8 \mathrm{~mL} / \mathrm{min} ; \mathrm{UV}$ detection at $210 \mathrm{~nm}$; $\mathrm{tR}=10.61 \mathrm{~min}$ (major), $16.07 \mathrm{~min}$ (minor). ${ }^{1} \mathrm{H} \mathrm{NMR}\left(600 \mathrm{MHz}, \mathrm{CDCl}_{3}\right)$ $\delta 7.55-7.48(\mathrm{~m}, 2 \mathrm{H}), 7.35-7.27(\mathrm{~m}, 5 \mathrm{H}), 7.15(\mathrm{ddd}, J=9.7,4.9,2.0 \mathrm{~Hz}, 1 \mathrm{H}), 6.14(\mathrm{t}, J=3.1 \mathrm{~Hz}, 1 \mathrm{H}), 2.55(\mathrm{t}, J=$ $3.4 \mathrm{~Hz}, 1 \mathrm{H}) .{ }^{13} \mathrm{C} \mathrm{NMR}\left(151 \mathrm{MHz}, \mathrm{CDCl}_{3}\right) \delta 142.2,140.6,133.6,133.0,129.4,128.6,128.6,128.4,128.4,127.9$,

122.7, 74.1.

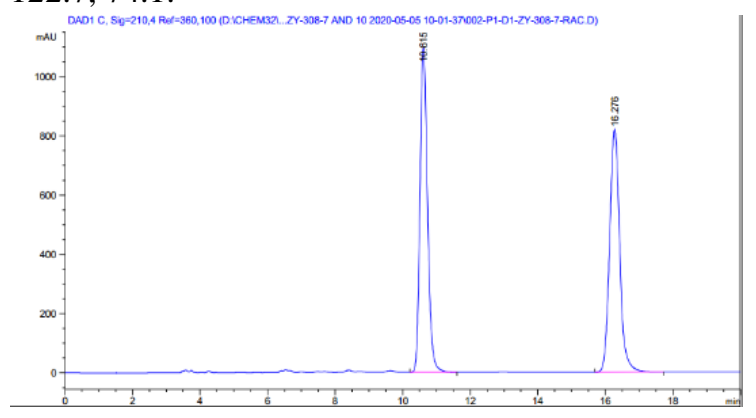

Signal 1: DAD1 C, Sig=210,4 Ref $=360,100$

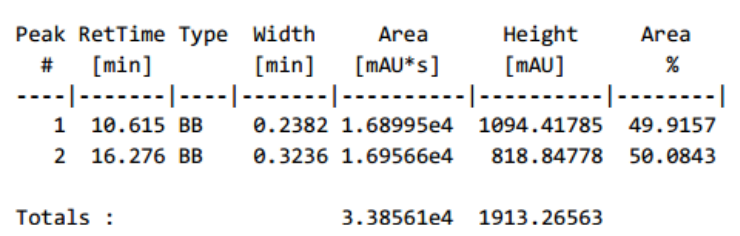

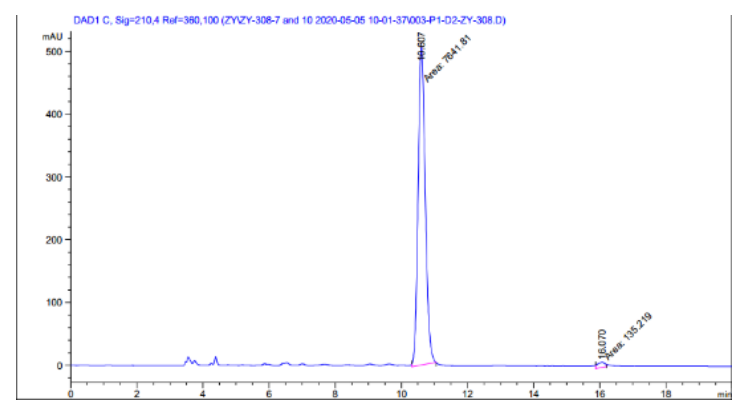

Signal 1: DAD1 C, Sig=210,4 Ref=360,100

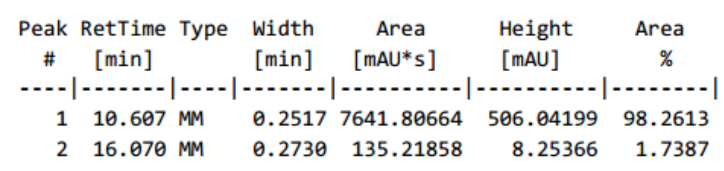


(2)-(2-bromophenyl)(4-fluorophenyl)methanol ${ }^{28}$ (101) Purified with flash chromatography on silica (PE:EA=8:1), Colorless oil, $11.4 \mathrm{mg}, 86 \%$ yield, $97 \%$ ee. $[\alpha]_{\mathrm{D}}^{24}=+29.6\left(\mathrm{c}=0.62, \mathrm{CHCl}_{3}\right)$; $\mathrm{The}^{24}$ enantiomeric excess was determined by UPLC on Chiralpak IB-U column, hexane:isopropanol $=95: 5$; flow rate $=0.5 \mathrm{~mL} / \mathrm{min}$; UV detection at $210 \mathrm{~nm}$; $\mathrm{tR}=3.16 \mathrm{~min}$ (major), $3.70 \mathrm{~min}$ (minor). ${ }^{1} \mathrm{H} \mathrm{NMR}\left(600 \mathrm{MHz}, \mathrm{CDCl}_{3}\right) \delta 7.56$ (dd, $J=7.9,2.4$ $\mathrm{Hz}, 1 \mathrm{H}), 7.53(\mathrm{dd}, J=8.1,2.6 \mathrm{~Hz}, 1 \mathrm{H}), 7.35(\mathrm{dq}, J=8.1,5.2,4.7 \mathrm{~Hz}, 3 \mathrm{H}), 7.15(\mathrm{td}, J=7.8,2.2 \mathrm{~Hz}, 1 \mathrm{H}), 7.04-6.97$ $(\mathrm{m}, 2 \mathrm{H}), 6.16(\mathrm{~d}, J=3.5 \mathrm{~Hz}, 1 \mathrm{H}), 2.45(\mathrm{~d}, J=3.5 \mathrm{~Hz}, 1 \mathrm{H}) .{ }^{13} \mathrm{C} \mathrm{NMR}\left(101 \mathrm{MHz}, \mathrm{CDCl}_{3}\right) \delta 162.3(\mathrm{~d}, J=247.5 \mathrm{~Hz})$, 142.4, $137.9(\mathrm{~d}, J=3.0 \mathrm{~Hz}), 132.9,129.3,128.8(\mathrm{~d}, J=8.1 \mathrm{~Hz}), 128.3,127.8,122.7,115.5,115.2,74.2 .{ }^{19} \mathrm{~F}$ NMR $\left(376 \mathrm{MHz}, \mathrm{CDCl}_{3}\right) \delta-114.5$.

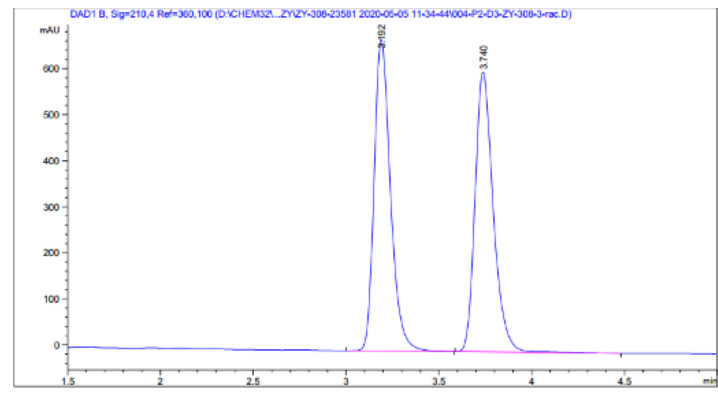

Signal 1: DAD1 B, Sig=210,4 Ref $=360,100$

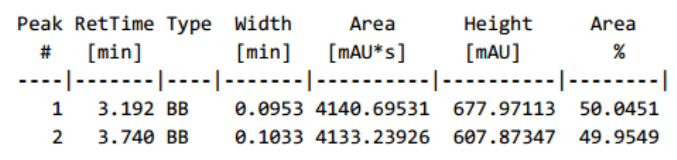

Totals : 8273.934571285 .84460

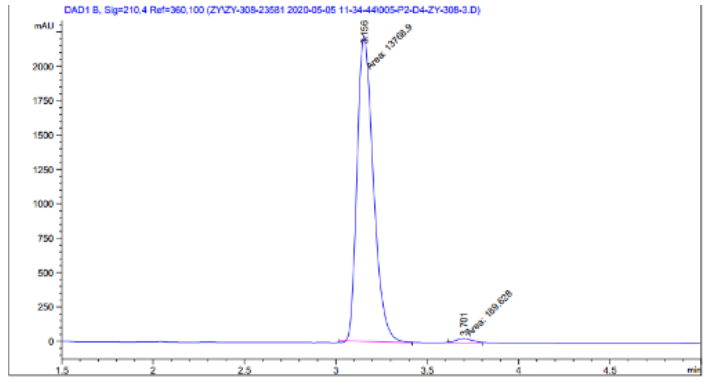

Signal 1: DAD1 B, Sig $=210,4$ Ref $=360,100$

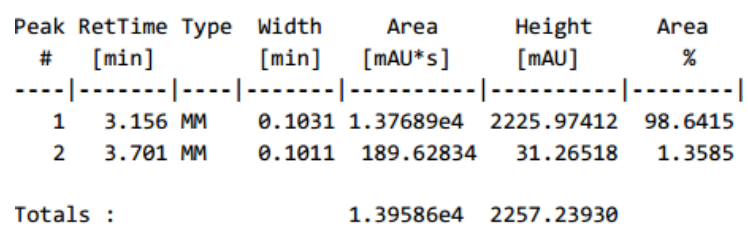


COOMe methyl (R)-4-((2-bromophenyl)(hydroxy)methyl)benzoate ${ }^{29}$ (10m) Purified with flash chromatography on silica (PE:EA=8:1), Colorless oil, $14.4 \mathrm{mg}, 90 \%$ yield, $97 \% e e .[\alpha]_{\mathrm{D}}{ }^{24}=+69.0(\mathrm{c}=0.71$, $\mathrm{CHCl}_{3}$ ); The enantiomeric excess was determined by UPLC on Chiralpak IC-U column, hexane:isopropanol = 95:5; flow rate $=0.5 \mathrm{~mL} / \mathrm{min} ; \mathrm{UV}$ detection at $210 \mathrm{~nm}$; $\mathrm{R}=7.31 \mathrm{~min}$ (major), $6.39 \mathrm{~min}$ (minor). ${ }^{1} \mathrm{H} \mathrm{NMR}\left(400 \mathrm{MHz}, \mathrm{CDCl}_{3}\right)$ $\delta 8.03-7.98(\mathrm{~m}, 2 \mathrm{H}), 7.55(\mathrm{dd}, J=8.0,1.3 \mathrm{~Hz}, 1 \mathrm{H}), 7.52-7.47(\mathrm{~m}, 3 \mathrm{H}), 7.34(\mathrm{td}, J=7.6,1.3 \mathrm{~Hz}, 1 \mathrm{H}), 7.20-7.13$ $(\mathrm{m}, 1 \mathrm{H}), 6.27(\mathrm{~d}, J=3.9 \mathrm{~Hz}, 1 \mathrm{H}), 3.90(\mathrm{~d}, J=1.4 \mathrm{~Hz}, 3 \mathrm{H}), 2.51(\mathrm{~d}, J=4.0 \mathrm{~Hz}, 1 \mathrm{H}) .{ }^{13} \mathrm{C} \mathrm{NMR}\left(101 \mathrm{MHz}, \mathrm{CDCl}_{3}\right) \delta$ $166.9,147.1,142.1,133.0,129.8,129.5,128.7,127.9,126.9,122.8,74.4,52.1$.

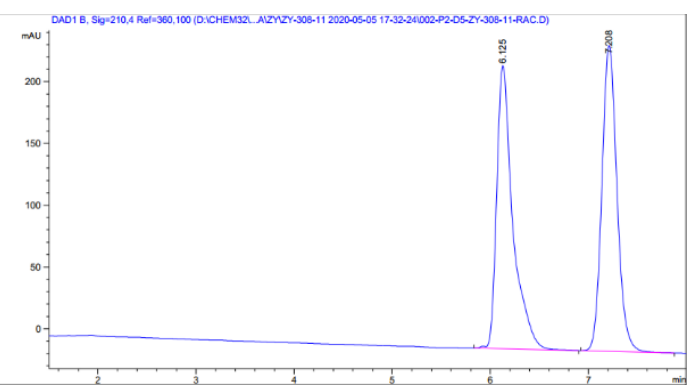

Signal 1: DAD1 B, Sig=210,4 Ref=360,100

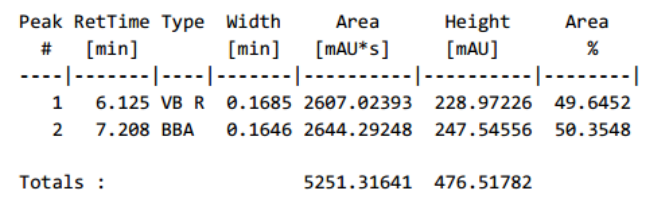

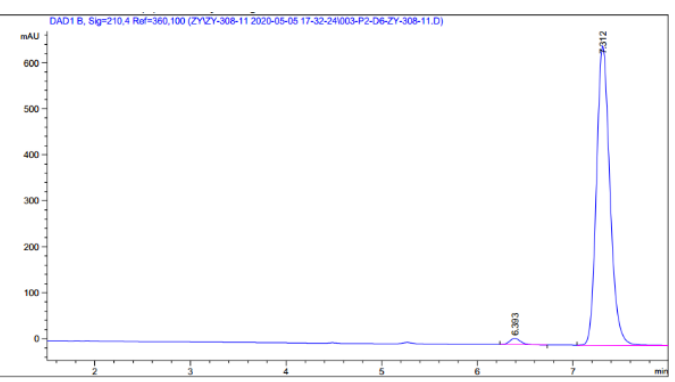

Signal 1: DAD1 B, Sig=210,4 Ref=360,100

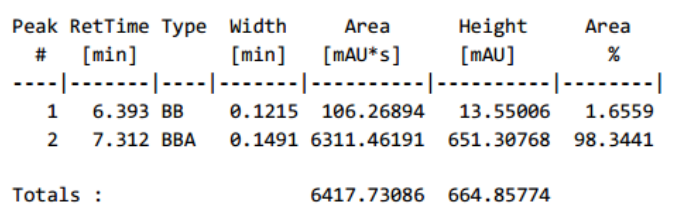


<smiles>OC(c1cccc(F)c1)c1ccccc1Br</smiles>

(R)-(2-bromophenyl)(4-methoxyphenyl)methanol ${ }^{30} \quad(10 n)$ Purified with flash chromatography on silica (PE:EA=8:1), Colorless oil, $12.4 \mathrm{mg}, 88 \%$ yield, $98 \%$ ee. $[\alpha]_{\mathrm{D}}^{24}=+55.5\left(\mathrm{c}=0.62, \mathrm{CHCl}_{3}\right)$; The enantiomeric excess was determined by UPLC on Chiralpak OD-3 column, hexane:isopropanol = 90:10; flow rate $=0.5 \mathrm{~mL} / \mathrm{min} ; \mathrm{UV}$ detection at $210 \mathrm{~nm} ; \mathrm{tR}=2.86 \mathrm{~min}$ (major), $4.66 \mathrm{~min}$ (minor). ${ }^{1} \mathrm{H} \mathrm{NMR}\left(400 \mathrm{MHz}, \mathrm{CDCl}_{3}\right) \delta$ $7.50(\mathrm{dd}, J=8.0,1.3 \mathrm{~Hz}, 1 \mathrm{H}), 7.45(\mathrm{dd}, J=7.8,1.7 \mathrm{~Hz}, 1 \mathrm{H}), 7.31-7.20(\mathrm{~m}, 2 \mathrm{H}), 7.16-7.03(\mathrm{~m}, 3 \mathrm{H}), 6.96-6.89$ $(\mathrm{m}, 1 \mathrm{H}), 6.09(\mathrm{~d}, J=3.5 \mathrm{~Hz}, 1 \mathrm{H}), 3.00(\mathrm{~d}, J=3.8 \mathrm{~Hz}, 1 \mathrm{H}) .{ }^{13} \mathrm{C} \mathrm{NMR}\left(101 \mathrm{MHz}, \mathrm{CDCl}_{3}\right) \delta 162.9(\mathrm{~d}, J=247.5 \mathrm{~Hz})$, $144.8(\mathrm{~d}, J=7.1 \mathrm{~Hz}), 142.1,132.9,130.0(\mathrm{~d}, J=8.1 \mathrm{~Hz}), 129.4,128.5,127.9,122.8,122.7$ (d, $J=3.0 \mathrm{~Hz}), 114.7$ (d, $J=21.2 \mathrm{~Hz}), 114.0(\mathrm{~d}, J=22.2 \mathrm{~Hz}), 74.1 .{ }^{19} \mathrm{~F} \mathrm{NMR}\left(376 \mathrm{MHz}, \mathrm{CDCl}_{3}\right) \delta-112.7$.

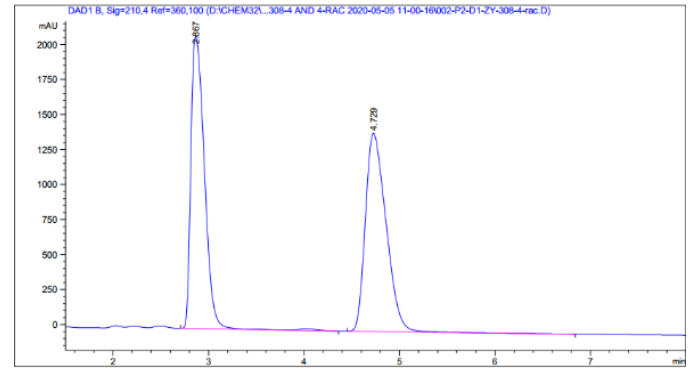

Signal 1: DAD1 B, Sig=210,4 Ref $=360,100$

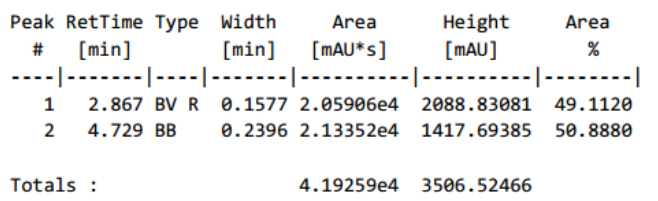

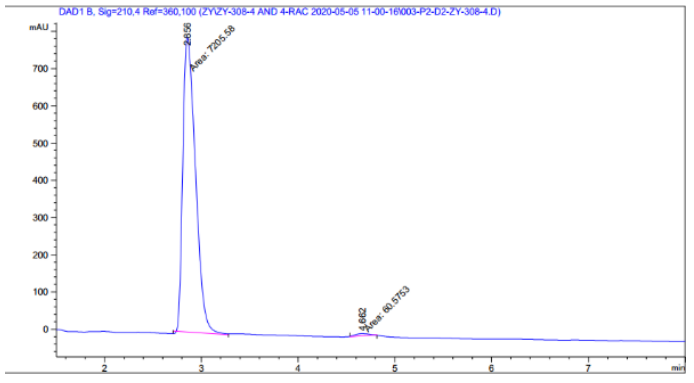

Signal 1: DAD1 B, Sig=210,4 Ref $=360,100$

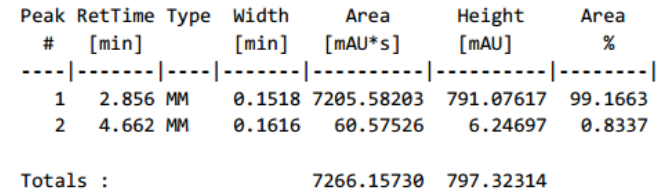


<smiles>CC#Cc1ccc([C@@H](O)c2ccccc2C)cc1</smiles>

(R)-(4-(phenylethynyl)phenyl)(o-tolyl)methanol (100) Purified with flash chromatography on silica (PE:EA=8:1), Green oil, $7.5 \mathrm{mg}, 74 \%$ yield, $97 \%$ ee. $[\alpha]_{\mathrm{D}}^{24}=+29.4\left(\mathrm{c}=0.34, \mathrm{CHCl}_{3}\right)$; The enantiomeric excess was determined by UPLC on Chiralpak IC-U column, hexane:isopropanol $=95: 5$; flow rate $=0.5$ $\mathrm{mL} / \mathrm{min}$; UV detection at $210 \mathrm{~nm}$; $\mathrm{tR}=2.95 \mathrm{~min}$ (major), $2.47 \mathrm{~min}$ (minor). ${ }^{1} \mathrm{H} \mathrm{NMR}\left(400 \mathrm{MHz}, \mathrm{CDCl}_{3}\right.$ ) $\delta 7.46-7.41$ (m, 2H), $7.41-7.37$ (m, 2H), $7.34(\mathrm{dd}, J=7.0,2.1 \mathrm{~Hz}, 1 \mathrm{H}), 7.23$ (qd, $J=4.2,1.4 \mathrm{~Hz}, 3 \mathrm{H}), 7.21-7.17$ (m, 2H), 7.16 $-7.08(\mathrm{~m}, 2 \mathrm{H}), 7.07-7.02(\mathrm{~m}, 1 \mathrm{H}), 5.86(\mathrm{~s}, 1 \mathrm{H}), 2.32(\mathrm{~s}, 1 \mathrm{H}), 2.14(\mathrm{~s}, 3 \mathrm{H}),{ }^{13} \mathrm{C} \mathrm{NMR}\left(101 \mathrm{MHz}, \mathrm{CDCl}_{3}\right) \delta 143.1$, 141.1, 135.5, 131.7, 131.7, 130.7, 128.4, 128.3, 127.8, 127.1, 126.5, 126.3, 123.2, 122.4, 89.6, 89.3, 73.1, 19.4. $\mathrm{m} / \mathrm{z}$ (ESI-MS): calc. $281.1325[\mathrm{M}-\mathrm{OH}]^{+}$, found $281.1323[\mathrm{M}-\mathrm{OH}]^{+}$.
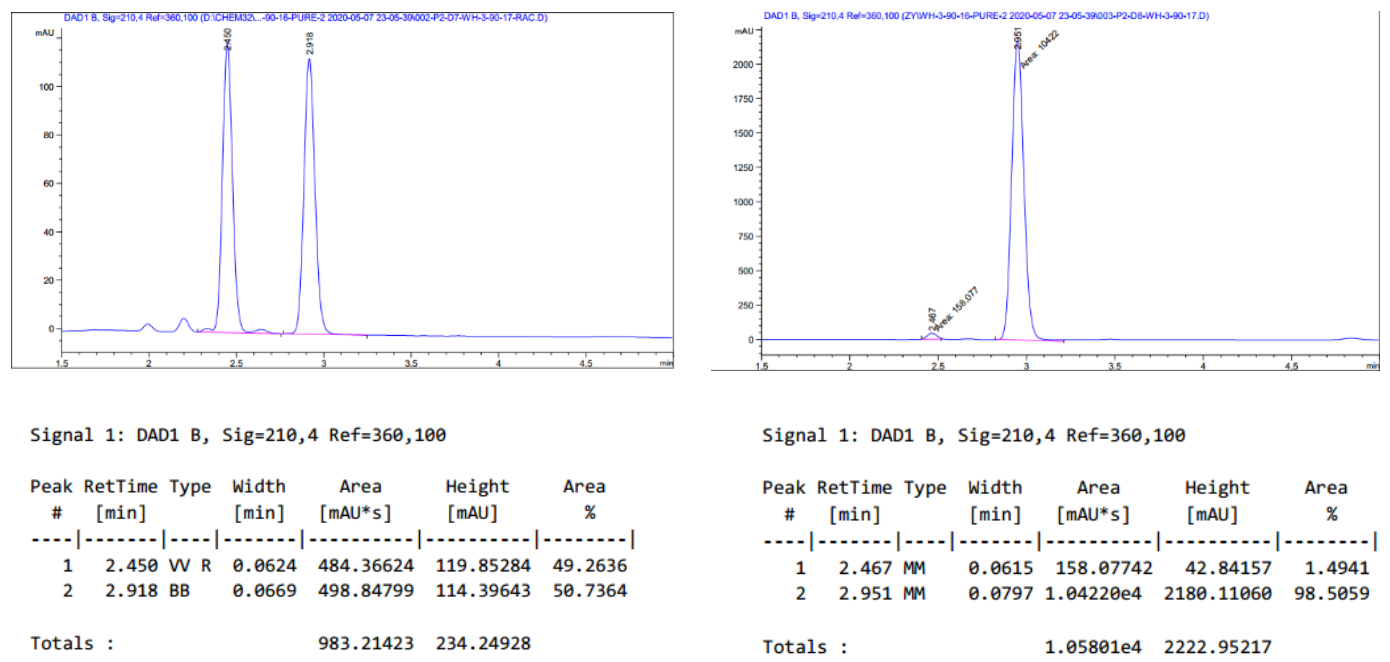


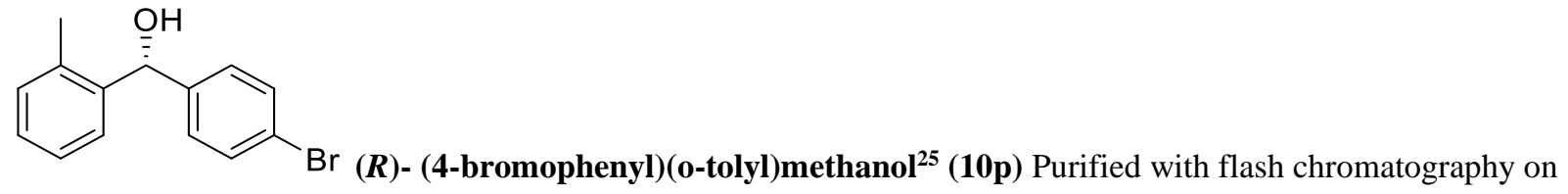
silica (PE:EA=8:1), Colorless oil, $12.9 \mathrm{mg}, 93 \%$ yield, $96 \%$ ee. $[\alpha]_{\mathrm{D}}{ }^{24}=+0.9\left(\mathrm{c}=0.64, \mathrm{CHCl}_{3}\right)$; The enantiomeric excess was determined by UPLC on Chiralpak IC-U column, hexane:isopropanol $=95: 5$; flow rate $=0.5 \mathrm{~mL} / \mathrm{min}$; $\mathrm{UV}$ detection at $210 \mathrm{~nm}$; $\mathrm{tR}=2.41 \mathrm{~min}$ (major), $2.13 \mathrm{~min}$ (minor). ${ }^{1} \mathrm{H} \mathrm{NMR}\left(400 \mathrm{MHz}, \mathrm{CDCl}_{3}\right) \delta 7.35$ (ddd, $J=7.0$, 3.4, $1.6 \mathrm{~Hz}, 3 \mathrm{H}), 7.18-7.04(\mathrm{~m}, 5 \mathrm{H}), 5.86(\mathrm{~s}, 1 \mathrm{H}), 2.29-2.17(\mathrm{~m}, 1 \mathrm{H}), 2.16(\mathrm{~s}, 3 \mathrm{H}) .{ }^{13} \mathrm{C} \mathrm{NMR}\left(101 \mathrm{MHz}, \mathrm{CDCl}_{3}\right) \delta$ $141.9,141.0,135.4,131.5,130.7,128.8,127.8,126.4,126.3,121.5,72.8,19.4$.
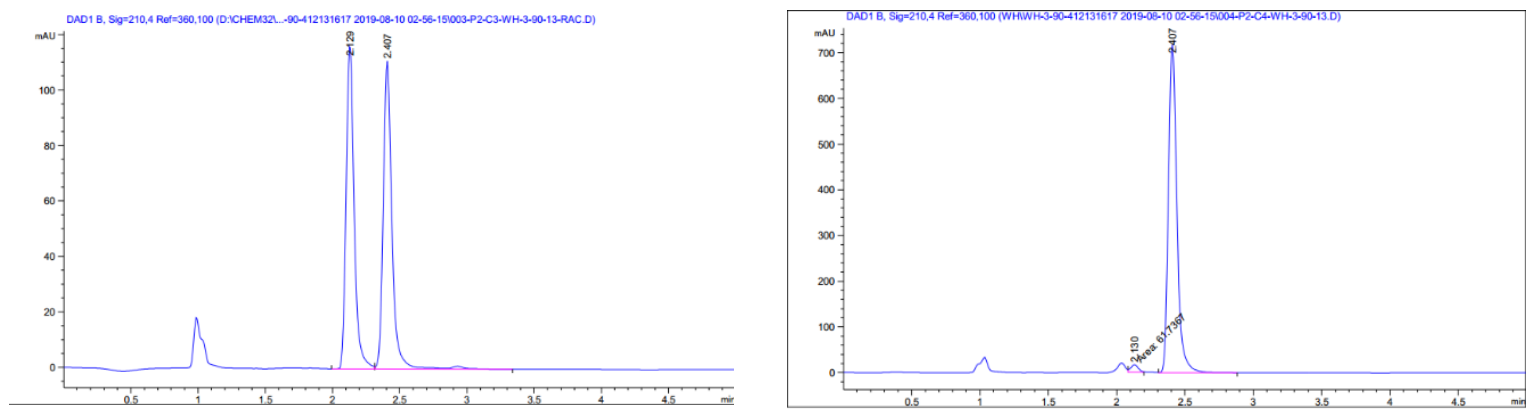

Signal 1: DAD1 B, Sig=210,4 Ref $=360,100$

Signal 1: DAD1 B, Sig=210,4 Ref $=360,100$
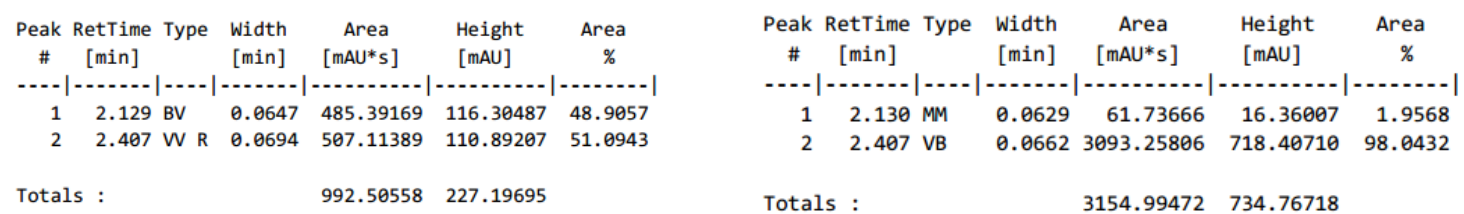
<smiles>Cc1ccccc1[C@@H](O)c1ccc(C(F)(F)F)cc1</smiles>

$(R)$-o-tolyl(4-(trifluoromethyl)phenyl)methanol ${ }^{26} \quad(10 q) \quad$ Purified with flash chromatography on silica (PE:EA=8:1), Colorless oil, $12.1 \mathrm{mg}, 91 \%$ yield, $97 \%$ ee. $[\alpha]_{\mathrm{D}}{ }^{24}=+26.6\left(\mathrm{c}=0.61, \mathrm{CHCl}_{3}\right)$; The enantiomeric excess was determined by UPLC on Chiralpak OD-H column, hexane:isopropanol = 90:10; flow rate $=0.8 \mathrm{~mL} / \mathrm{min} ; \mathrm{UV}$ detection at $210 \mathrm{~nm}$; $\mathrm{tR}=11.87 \mathrm{~min}$ (major), $13.13 \mathrm{~min}$ (minor). ${ }^{1} \mathrm{H} \mathrm{NMR}$ (400 $\mathrm{MHz}, \mathrm{CDCl}_{3}$ ) $\delta 7.49(\mathrm{~d}, J=8.1 \mathrm{~Hz}, 2 \mathrm{H}), 7.35(\mathrm{~s}, 2 \mathrm{H}), 7.33-7.27(\mathrm{~m}, 1 \mathrm{H}), 7.19-7.13(\mathrm{~m}, 2 \mathrm{H}), 7.08(\mathrm{dt}, J=4.3 \mathrm{~Hz}, 3.2 \mathrm{~Hz}, 1 \mathrm{H})$, $5.95(\mathrm{~d}, J=3.1 \mathrm{~Hz}, 1 \mathrm{H}), 2.27(\mathrm{~s}, 1 \mathrm{H}), 2.19(\mathrm{~s}, 3 \mathrm{H}) .{ }^{13} \mathrm{C} \mathrm{NMR}\left(101 \mathrm{MHz}, \mathrm{CDCl}_{3}\right) \delta 146.81,140.82,135.54,130.86$, 129.3(q, $J=41.4 \mathrm{~Hz}), 128.06,127.20,126.67,126.39,125.37$ (q, $J=3.7 \mathrm{~Hz}), 124.1(\mathrm{q}, J=273.7 \mathrm{~Hz}), 72.86,19.39$. ${ }^{19} \mathrm{~F}$ NMR $\left(376 \mathrm{MHz}, \mathrm{CDCl}_{3}\right) \delta-62.6$.

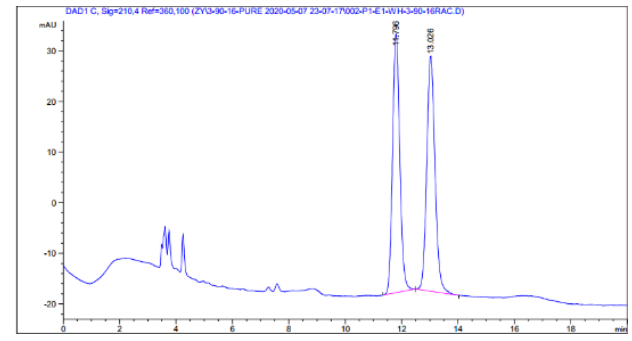

Signal 1: DAD1 C, Sig $=210,4$ Ref $=360,100$

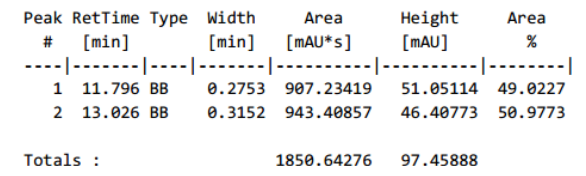

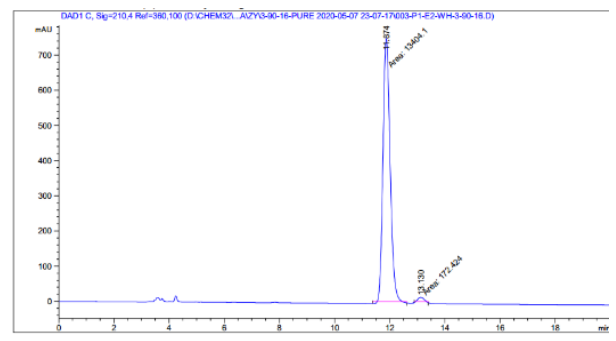

Signal 1: DAD1 C, Sig $=210,4$ Ref $=360,100$

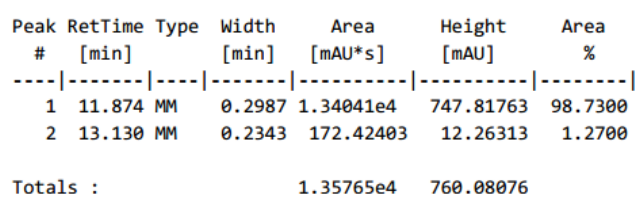


OMe $(R)$-(4-methoxyphenyl)(o-tolyl)methanol ${ }^{27}$ (10r) Purified with flash chromatography on silica (PE:EA=8:1), White solid, $4.0 \mathrm{mg}$, 35\% yield, 95\% ee. $[\alpha]_{\mathrm{D}}{ }^{24}=-5.63(\mathrm{c}=0.12$, THF); The enantiomeric excess was determined by UPLC on Chiralpak IC-U column, hexane:isopropanol $=95: 5$; flow rate $=0.5 \mathrm{~mL} / \mathrm{min}$; UV detection at $210 \mathrm{~nm}$; tR = $5.28 \mathrm{~min}$ (major), $4.26 \mathrm{~min}$ (minor). ${ }^{1} \mathrm{H} \mathrm{NMR}\left(400 \mathrm{MHz}, \mathrm{CHCl}_{3}\right) \delta 7.47$ (dd, $J=7.6 \mathrm{~Hz}, 1.5$ $\mathrm{Hz}, 1 \mathrm{H}), 7.19-7.05(\mathrm{~m}, 4 \mathrm{H}), 7.05-7.01(\mathrm{~m}, 1 \mathrm{H}), 6.78-6.72(\mathrm{~m}, 2 \mathrm{H}), 5.87-5.79(\mathrm{~m}, 1 \mathrm{H}), 3.68(\mathrm{~s}, 3 \mathrm{H}), 2.17(\mathrm{~d}, J=$ $3.0 \mathrm{~Hz}, 1 \mathrm{H}), 2.11(\mathrm{~s}, 3 \mathrm{H}) .{ }^{13} \mathrm{C} \mathrm{NMR}\left(101 \mathrm{MHz}, \mathrm{CDCl}_{3}\right) \delta 159.0,141.7,135.2,135.1,130.5,128.5,127.4,126.1,125.9$, $113.9,72.9,55.3,19.4$.
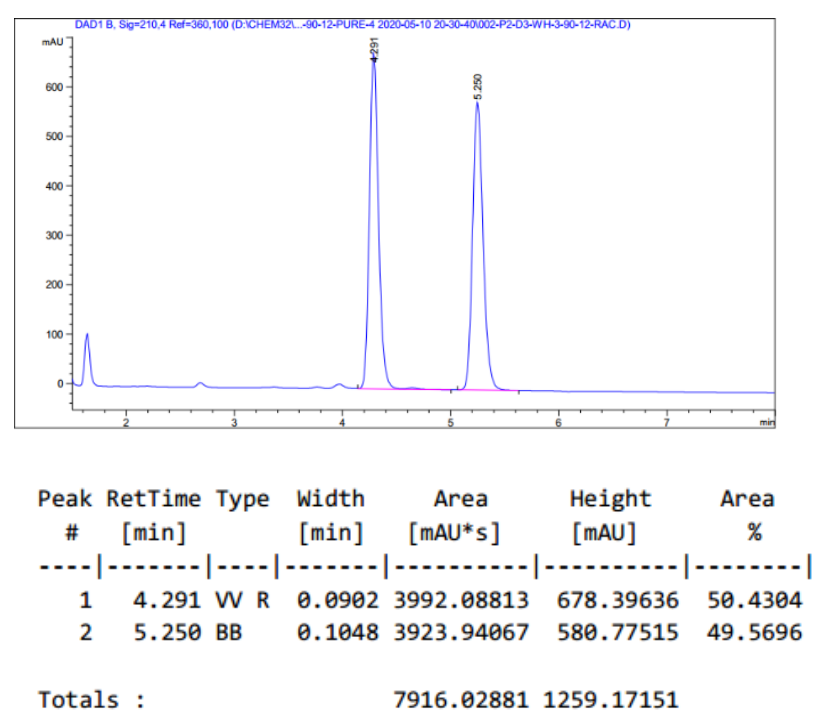

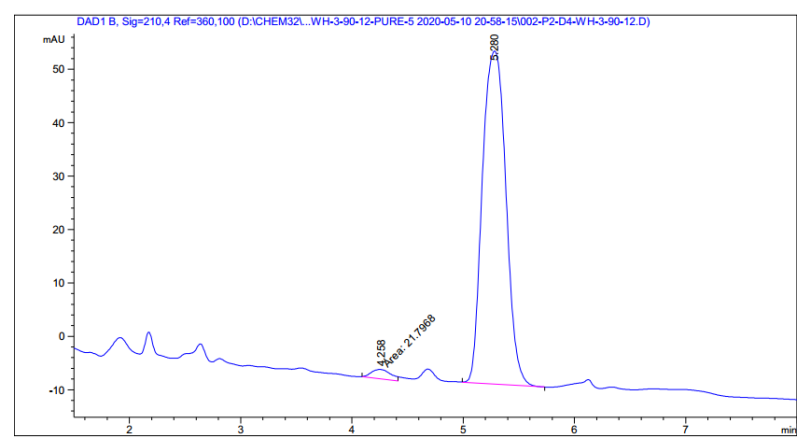

Signal 1: DAD1 B, Sig=210,4 Ref $=360,100$

\begin{tabular}{|c|c|c|c|c|c|c|}
\hline $\begin{array}{c}\text { Peak } \\
\#\end{array}$ & $\begin{array}{c}\text { RetTime } \\
\text { [min] }\end{array}$ & Type & $\begin{array}{l}\text { Width } \\
\text { [min] }\end{array}$ & $\begin{array}{c}\text { Area } \\
{[\mathrm{mAU} * \mathrm{~s}]}\end{array}$ & $\begin{array}{l}\text { Height } \\
\text { [mAU] }\end{array}$ & $\begin{array}{c}\text { Area } \\
\%\end{array}$ \\
\hline$\cdots$ & | & & | & - & - & 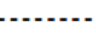 \\
\hline 1 & 4.258 & MM & 0.2061 & 21.79685 & 1.76224 & 2.3216 \\
\hline 2 & 5.280 & VB & 0.2396 & 917.07281 & 62.35664 & 97.6784 \\
\hline Tota] & ls : & & & 938.86966 & 64.11888 & \\
\hline
\end{tabular}


<smiles>OC(c1ccccc1)c1ccc(Cl)cc1Br</smiles>

(R)-(2-bromo-4-chlorophenyl)(phenyl)methanol ${ }^{24} \quad$ (10s) Purified with flash chromatography on silica (PE:EA=8:1), white oil, $13.0 \mathrm{mg}, 87 \%$ yield, $96 \%$ ee. $[\alpha]_{\mathrm{D}}^{24}=-20.4\left(\mathrm{c}=0.56, \mathrm{CHCl}_{3}\right)$; The enantiomeric excess was determined by UPLC on Chiralpak IB-U column, hexane:isopropanol $=95: 5$; flow rate $=0.8$ $\mathrm{mL} / \mathrm{min}$; UV detection at $210 \mathrm{~nm}$; tR $=5.21 \mathrm{~min}$ (major), $5.54 \mathrm{~min}$ (minor). ${ }^{1} \mathrm{H} \mathrm{NMR}\left(600 \mathrm{MHz}, \mathrm{CDCl}_{3}\right) \delta 7.53(\mathrm{~d}, J$ $=7.8 \mathrm{~Hz}, 1 \mathrm{H}), 7.35-7.31(\mathrm{~m}, 1 \mathrm{H}), 7.25(\mathrm{dp}, J=16.3,7.7 \mathrm{~Hz}, 4 \mathrm{H}), 7.08(\mathrm{dt}, J=7.7,4.6 \mathrm{~Hz}, 1 \mathrm{H}), 7.03(\mathrm{t}, J=9.1 \mathrm{~Hz}$, $1 \mathrm{H}), 6.45(\mathrm{~d}, J=3.4 \mathrm{~Hz}, 1 \mathrm{H}), 2.68(\mathrm{t}, J=3.3 \mathrm{~Hz}, 1 \mathrm{H}) .{ }^{13} \mathrm{C} \mathrm{NMR}\left(101 \mathrm{MHz}, \mathrm{CDCl}_{3}\right) \delta 141.7,141.1,134.0,132.4$, $129.2,128.6,128.0,128.0,127.0,122.9,74.3$.
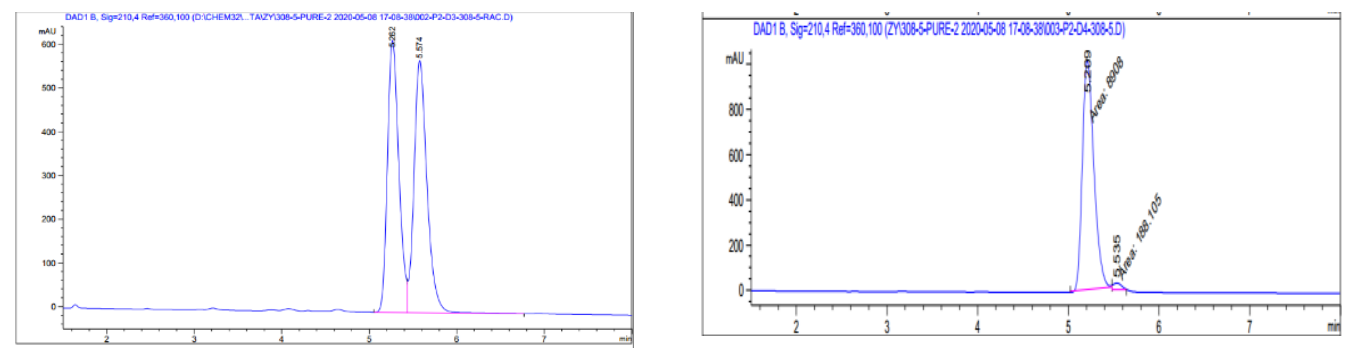

Signal 1: DAD1 B, Sig=210,4 Ref=360,100

Signal 1: DAD1 B, Sig=210,4 Ref=360,100
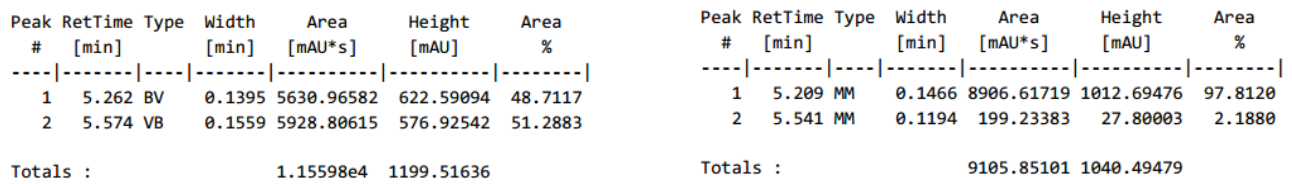
<smiles>O[C@@H](c1ccccc1F)c1ccccc1Cl</smiles>

(R)-(2-chlorophenyl)(2-fluorophenyl)methanol ${ }^{12}$ (10t) Purified with flash chromatography on silica (PE:EA=8:1), Colorless oil, $12.7 \mathrm{mg}, 87 \%$ yield, $90 \%$ ee. $[\alpha]_{\mathrm{D}}^{24}=-4.2\left(\mathrm{c}=0.43, \mathrm{CHCl}_{3}\right)$; The enantiomeric excess was determined by UPLC on Chiralpak OD-H column, hexane:isopropanol $=90: 10$; flow rate $=0.8 \mathrm{~mL} / \mathrm{min}$; $\mathrm{UV}$ detection at $210 \mathrm{~nm}$; $\mathrm{R}=11.11 \mathrm{~min}$ (major), $13.21 \mathrm{~min}$ (minor). ${ }^{1} \mathrm{H} \mathrm{NMR}\left(400 \mathrm{MHz}, \mathrm{CDCl}_{3}\right) \delta 7.49-7.42(\mathrm{~m}$, 2H), $7.28-7.17(\mathrm{~m}, 6 \mathrm{H}), 6.02(\mathrm{~s}, 1 \mathrm{H}), 2.47(\mathrm{~s}, 1 \mathrm{H}) .{ }^{13} \mathrm{C} \mathrm{NMR}\left(151 \mathrm{MHz}, \mathrm{CDCl}_{3}\right) \delta 160.4(\mathrm{~d}, J=247.6 \mathrm{~Hz}), 139.6$, 132.7, 129.6 (d, $J=7.6 \mathrm{~Hz}) 129.6,129.2,129.1$ (d, $J=6.04 \mathrm{~Hz}), 128.5(\mathrm{~d}, J=3.02 \mathrm{~Hz}), 128.1,127.0,124.2,124.2$, 115.5, (d, $J=19.6 \mathrm{~Hz}), 67.0(\mathrm{~d}, J=4.5 \mathrm{~Hz}) .{ }^{19} \mathrm{~F}$ NMR $\left(376 \mathrm{MHz}, \mathrm{CDCl}_{3}\right) \delta-117.9$.

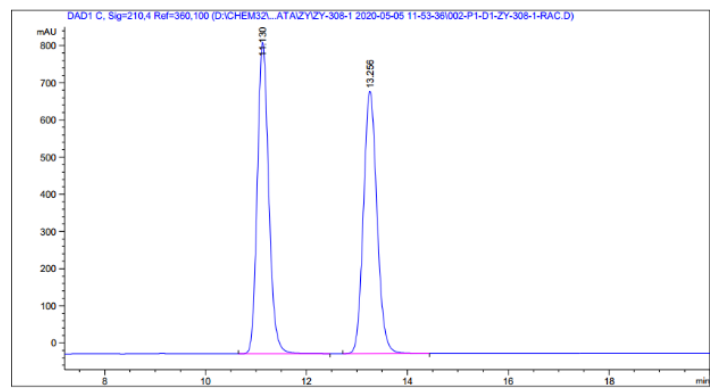

Signal 1: DAD1 C, Sig=210,4 Ref $=360,100$

\begin{tabular}{|c|c|c|c|c|c|}
\hline $\begin{array}{c}\text { Peak } \\
\#\end{array}$ & $\begin{array}{l}\text { RetTime Type } \\
\text { [min] }\end{array}$ & $\begin{array}{l}\text { Width } \\
\text { [min] }\end{array}$ & $\begin{array}{c}\text { Area } \\
{[\mathrm{mAU} * \mathrm{~s}]}\end{array}$ & $\begin{array}{l}\text { Height } \\
\text { [mAU] }\end{array}$ & $\begin{array}{c}\text { Area } \\
\%\end{array}$ \\
\hline$\cdots$ & 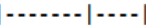 & 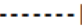 & | - & | & | \\
\hline 1 & $11.130 \mathrm{BB}$ & 0.2433 & $1.31580 \mathrm{e} 4$ & 837.38654 & 49.9776 \\
\hline 2 & $13.256 \mathrm{BB}$ & 0.2897 & $1.31698 \mathrm{e} 4$ & 705.73248 & 50.0224 \\
\hline Total & & & $2.63278 \mathrm{e} 4$ & 1543.11902 & \\
\hline
\end{tabular}

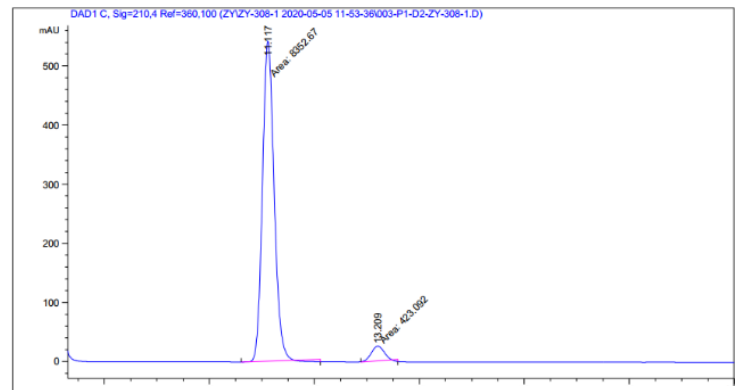

Signal 1: DAD1 C, Sig=210,4 Ref=360,100

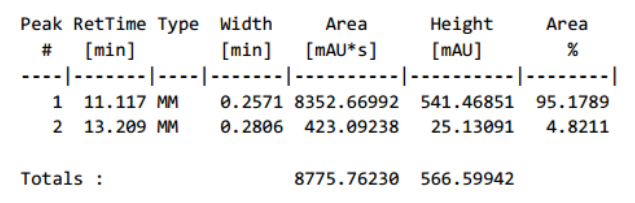


<smiles>O[C@@H](c1ccccc1F)c1ccccc1I</smiles>

(R)-(2-fluorophenyl)(2-iodophenyl)methanol ${ }^{20}$ (10u) Purified with flash chromatography on silica (PE:EA=8:1), Colorless oil, $14.8 \mathrm{mg}, 90 \%$ yield, 93\% ee, $[\alpha]_{\mathrm{D}}{ }^{24}=-8.4\left(\mathrm{c}=0.32, \mathrm{CHCl}_{3}\right)$; The enantiomeric excess was determined by UPLC on Chiralpak IB-U column, hexane:isopropanol $=97: 3$; flow rate $=0.5 \mathrm{~mL} / \mathrm{min}$; UV detection at $210 \mathrm{~nm}$; $\mathrm{tR}=5.75 \mathrm{~min}$ (major), $7.37 \mathrm{~min}$ (minor). ${ }^{1} \mathrm{H} \mathrm{NMR}\left(400 \mathrm{MHz}, \mathrm{CDCl}_{3}\right) \delta 7.83$ (dd, $J=7.9,1.2$ $\mathrm{Hz}, 1 \mathrm{H}), 7.47$ (dd, $J=7.8,1.7 \mathrm{~Hz}, 1 \mathrm{H}), 7.36(\mathrm{td}, J=7.5,1.2 \mathrm{~Hz}, 1 \mathrm{H}), 7.28(\mathrm{qd}, J=5.5,3.4 \mathrm{~Hz}, 1 \mathrm{H}), 7.25-7.18(\mathrm{~m}$, 1H), $7.13-7.04(\mathrm{~m}, 2 \mathrm{H}), 7.04-6.96(\mathrm{~m}, 1 \mathrm{H}), 6.26(\mathrm{~d}, J=4.1 \mathrm{~Hz}, 1 \mathrm{H}), 2.62(\mathrm{dd}, J=4.4,1.5 \mathrm{~Hz}, 1 \mathrm{H}) .{ }^{13} \mathrm{C}$ NMR $(151$ $\left.\mathrm{MHz}, \mathrm{CDCl}_{3}\right) \delta 160.7(\mathrm{~d}, J=247.6 \mathrm{~Hz}) 159.8,143.9,139.7,129.7,(\mathrm{~d}, J=10.6 \mathrm{~Hz}), 129.1(\mathrm{~d}, J=13.6 \mathrm{~Hz}), 128.6(\mathrm{~d}$, $J=4.53 \mathrm{~Hz}), 128.5,128.3,124.2(\mathrm{~d}, J=3.0 \mathrm{~Hz}), 115.5,115.4,98.7,77.3,77.1,76.9,73.5,73.5 .{ }^{19} \mathrm{~F} \mathrm{NMR}(376 \mathrm{MHz}$, $\left.\mathrm{CDCl}_{3}\right) \delta-117.1$.

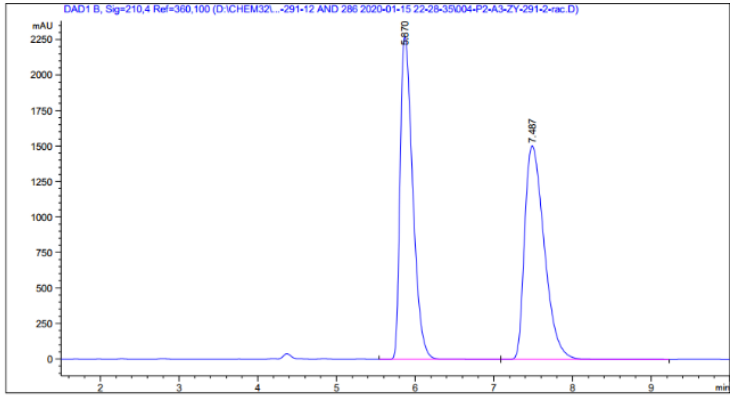

Signal 1: DAD1 B, Sig $=210,4$ Ref $=360,100$

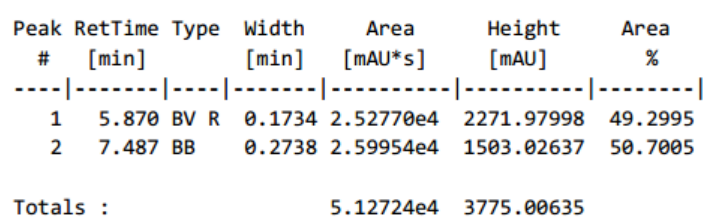

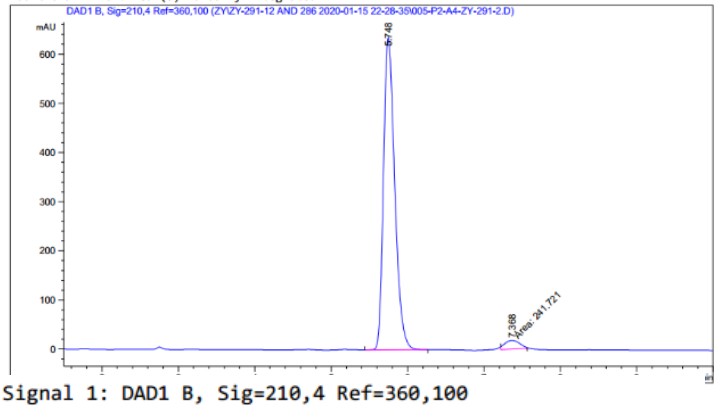

Peak RetTime Type Width Area Height Area

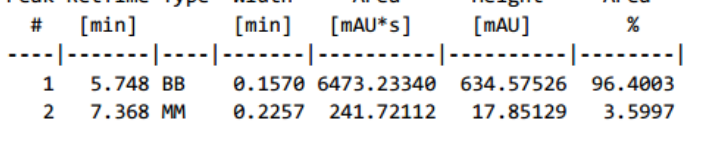

Totals : 
(R)-(2-bromophenyl)(2-fluorophenyl)methanol ${ }^{22}$ (10v) Purified with flash chromatography on silica (PE:EA=8:1), Colorless oil, $13.3 \mathrm{mg}, 95 \%$ yield, 93\% ee, $[\alpha]_{\mathrm{D}}{ }^{24}=+14.6\left(\mathrm{c}=0.42, \mathrm{CHCl}_{3}\right)$; The enantiomeric excess was determined by UPLC on Chiralpak IB-U column, hexane:isopropanol $=97: 3$; flow rate $=0.5 \mathrm{~mL} / \mathrm{min}$; UV detection at $210 \mathrm{~nm}$; $\mathrm{tR}=6.87 \mathrm{~min}$ (major), $8.83 \mathrm{~min}$ (minor). ${ }^{1} \mathrm{H} \mathrm{NMR}\left(600 \mathrm{MHz}, \mathrm{CDCl}_{3}\right) \delta 7.46$ (dd, $J=8.1,5.9$ $\mathrm{Hz}, 2 \mathrm{H}), 7.24(\mathrm{t}, J=7.6 \mathrm{~Hz}, 1 \mathrm{H}), 7.21-7.14(\mathrm{~m}, 2 \mathrm{H}), 7.07(\mathrm{t}, J=7.7 \mathrm{~Hz}, 1 \mathrm{H}), 6.99(\mathrm{dt}, J=26.9,8.3 \mathrm{~Hz}, 2 \mathrm{H}), 6.30$ (s, 1H), 3.26 (s, 1H). ${ }^{13} \mathrm{C}$ NMR $\left(151 \mathrm{MHz}, \mathrm{CDCl}_{3}\right) \delta 160.5(\mathrm{~d}, J=247.6 \mathrm{~Hz}), 141.2,132.9,129.6(\mathrm{~d}, J=7.6 \mathrm{~Hz})$, 129.3, $129.2(\mathrm{~d}, J=12.1 \mathrm{~Hz}), 128.6(\mathrm{~d}, J=3.0 \mathrm{~Hz}), 128.5,127.6,124.5(\mathrm{~d}, J=3.0 \mathrm{~Hz}), 122.9,115.6,115.4,69.1(\mathrm{~d}$, $J=4.5 \mathrm{~Hz}) .{ }^{19} \mathrm{~F}$ NMR $(376 \mathrm{MHz}$, Chloroform- $d$ ) $\delta-117.6$.

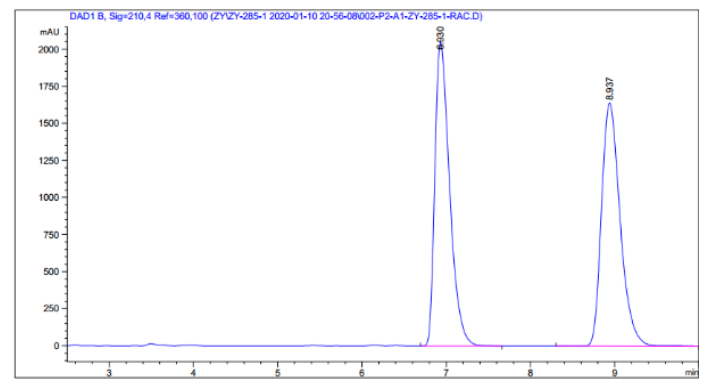

Signal 1: DAD1 B, Sig=210,4 Ref $=360,100$

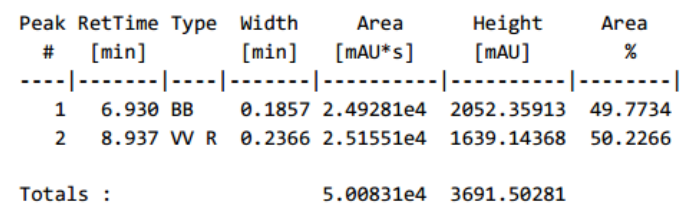

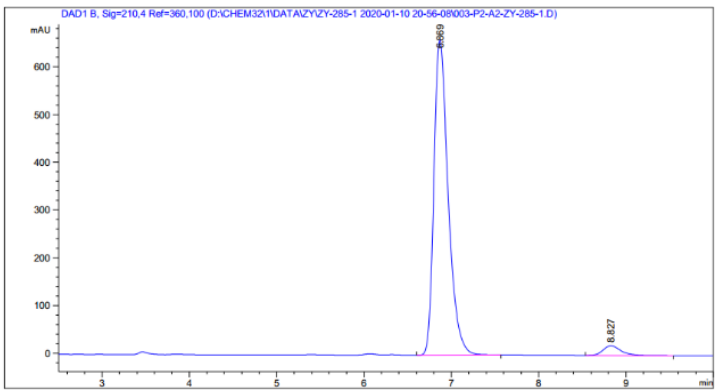

Signal 1: DAD1 B, Sig $=210,4$ Ref $=360,100$

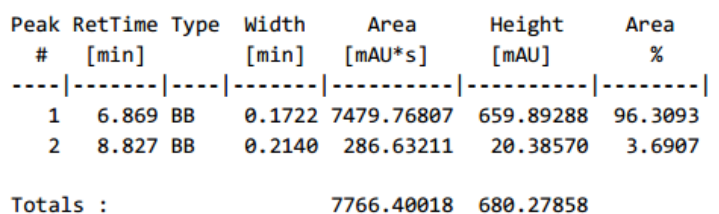


<smiles>COc1ccccc1[C@@H](O)c1ccccc1Br</smiles>

(R)-(2-bromophenyl)(2-methoxyphenyl)methanol ${ }^{23} \quad$ (10w) Purified with flash chromatography on silica (PE:EA=8:1), Colorless oil, $13.2 \mathrm{mg}$, 93\% yield, $90 \%$ ee. $[\alpha]_{\mathrm{D}}^{24}=+26.2\left(\mathrm{c}=0.50, \mathrm{CHCl}_{3}\right)$; The enantiomeric excess was determined by UPLC on Chiralpak IB-U column, hexane:isopropanol = 97:3; flow rate $=0.5 \mathrm{~mL} / \mathrm{min}$; UV detection at $210 \mathrm{~nm}$; $\mathrm{tR}=7.36 \mathrm{~min}$ (major), $8.48 \mathrm{~min}$ (minor). ${ }^{1} \mathrm{H} \mathrm{NMR}$ (400 MHz, $\mathrm{CDCl}_{3}$ ) $\delta 7.56$ (ddd, $J=7.9,6.3,1.5 \mathrm{~Hz}, 2 \mathrm{H}), 7.35(\mathrm{td}, J=7.5,1.3 \mathrm{~Hz}, 1 \mathrm{H}), 7.32-7.25(\mathrm{~m}, 1 \mathrm{H}), 7.17$ (dd, $J=7.7,1.8 \mathrm{~Hz}, 1 \mathrm{H}), 6.96$ $-6.86(\mathrm{~m}, 3 \mathrm{H}), 6.40(\mathrm{~d}, J=3.9 \mathrm{~Hz}, 1 \mathrm{H}), 3.88(\mathrm{~s}, 3 \mathrm{H}), 3.09(\mathrm{~d}, J=4.0 \mathrm{~Hz}, 1 \mathrm{H}) .{ }^{13} \mathrm{C} \mathrm{NMR}\left(101 \mathrm{MHz}, \mathrm{CDCl}_{3}\right) \delta 157.2$, $141.4,132.7,130.4,129.1,128.9,128.9,127.8,127.5,123.2,120.7,110.6,70.7,55.5$.

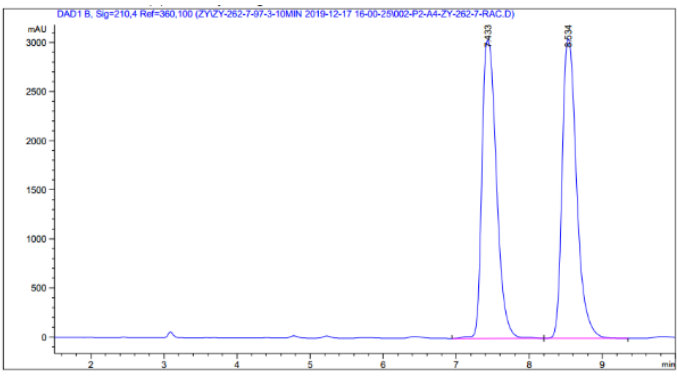

Signal 1: DAD1 B, Sig=210,4 Ref=360,100

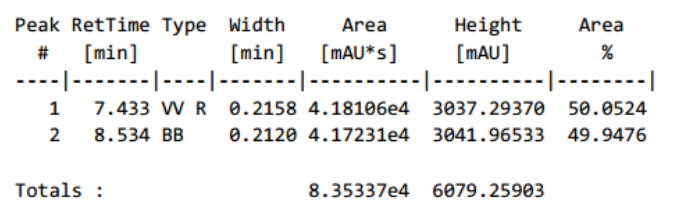

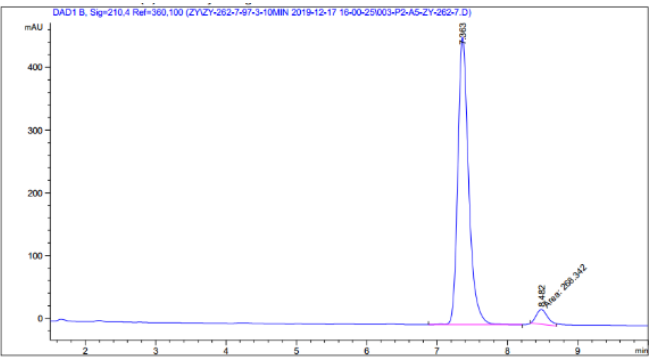

Signal 1: DAD1 B, Sig=210,4 Ref $=360,100$

\begin{tabular}{|c|c|c|c|c|c|c|}
\hline $\begin{array}{c}\text { Peak } \\
\#\end{array}$ & $\begin{array}{c}\text { RetTime } \\
\text { [min] }\end{array}$ & Type & $\begin{array}{l}\text { Width } \\
\text { [min] }\end{array}$ & $\begin{array}{c}\text { Area } \\
{\left[\mathrm{mAU}^{*} \mathrm{~s}\right]}\end{array}$ & $\begin{array}{l}\text { Height } \\
\text { [mAU] }\end{array}$ & $\begin{array}{c}\text { Area } \\
\%\end{array}$ \\
\hline$-\ldots \mid$ & .......... & & & 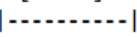 & | & | \\
\hline 1 & 7.363 & BB & 0.1628 & 4901.10791 & 458.32874 & 94.8091 \\
\hline 2 & 8.482 & MM & 0.1896 & 268.34225 & 23.58759 & 5.1909 \\
\hline Total & s : & & & 5169.45016 & 481.91633 & \\
\hline
\end{tabular}




\section{Synthesis and reactivity of $\left[\mathrm{FeBr}_{2}(\mathrm{Heng}-\mathrm{PNP})\right](6)$ and structural determination.}

Complex 6 was synthesized via modified reference mothod. ${ }^{5}$ The ligand Heng-PNP (43.7 mg, $0.1 \mathrm{mmol}$ ) was dissolved in $1 \mathrm{~mL}$ degassed THF at room temperature in a $10 \mathrm{~mL}$ Schlenk tube under argon. $28.5 \mathrm{mg} \mathrm{FeBr} \cdot \mathrm{THF}_{2}$ (0.08 mmol) in $0.6 \mathrm{~mL}$ degassed ethanol was added dropwise over a period of 5-10 minutes. A white cloudy precipitate 6 was formed and the mixture was stirred over night at room temperature. Carbon monoxide was bubbled through the suspension for 5-10 min until the precipitate was fully dissolved. A solution with dark purple color was formed. The solvents were removed under reduced pressure to obtain a pale purple powder solid, $45 \mathrm{mg}, 69 \%$ yield. The product was washed 3 times with $0.3 \mathrm{~mL}$ hexane and dried in vacuo. The crystal (6) was grown via natural volatilization of solvent in $\mathrm{CH}_{2} \mathrm{Cl}_{2} /$ hexane mixture. CCDC No. 2006388. Displacement ellipsoids are drawn at the 30\% probability level.
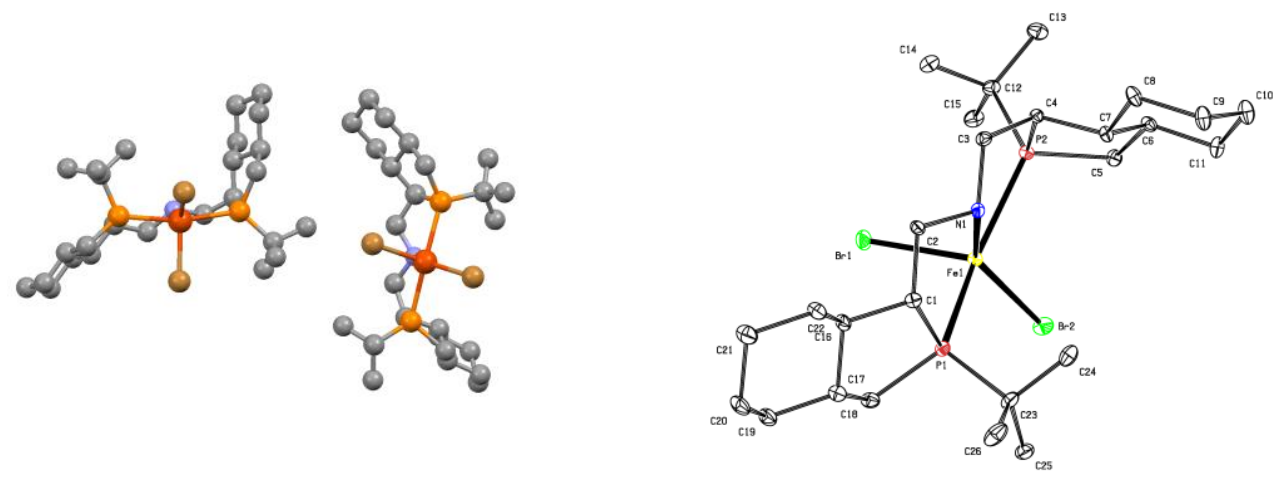

Identification code

Empirical formula

Formula weight

Temperature/K

Crystal system

cxy0626_0m

$\mathrm{C}_{26} \mathrm{H}_{49} \mathrm{Br}_{2} \mathrm{FeNP}_{2}$

653.27

100

Space group

monoclinic

$\mathrm{a} / \AA ̊$

$\mathrm{P} 21$

$\mathrm{b} / \AA$

7.2590(4)

20.0908(11)

$\mathrm{c} / \AA$

20.9420(11)

$\alpha /{ }^{\circ}$

90

$\beta /{ }^{\circ}$

93.992(2)

$\gamma /{ }^{\circ}$

90

Volume $/ \AA^{3}$

3046.8(3)

Z

4

$\rho_{\text {calc }} / \mathrm{cm}^{3}$

1.424

$\mu / \mathrm{mm}^{-1}$

3.237

$\mathrm{F}(000)$

1352.0

Crystal size $/ \mathrm{mm}^{3}$

$? \times ? \times$ ?

Radiation

$\mathrm{MoK} \alpha(\lambda=0.71073)$

$2 \Theta$ range for data collection/ ${ }^{\circ}$

4.5 to 55.086 
Index ranges

Reflections collected

Independent reflections

Data/restraints/parameters

Goodness-of-fit on $\mathrm{F}^{2}$

Final $\mathrm{R}$ indexes $[\mathrm{I}>=2 \sigma(\mathrm{I})]$

Final $\mathrm{R}$ indexes [all data]

Largest diff. peak/hole / e $\AA^{-3}$

Flack parameter
$-9 \leq \mathrm{h} \leq 9,-26 \leq \mathrm{k} \leq$

$26,-27 \leq 1 \leq 27$

44264

$14006\left[\mathrm{R}_{\mathrm{int}}=0.0597\right.$,

$\left.\mathrm{R}_{\text {sigma }}=0.0621\right]$

14006/1/589

1.020

$\mathrm{R}_{1}=0.0337, \mathrm{wR}_{2}=$

0.0740

$\mathrm{R}_{1}=0.0409, \mathrm{wR}_{2}=$ 0.0767

$0.69 /-0.57$

$0.009(4)$ 


\section{Computation studies.}

All the structures were optimized in the n-hexane by the PCM model ${ }^{34}$ using the Gaussian 09 D.01 program. ${ }^{35}$ Structures are optimized using the wb97xd functional ${ }^{36}$ with the basis set Lanl2dz for Ir, and 6-31G* for other main group atoms. ${ }^{37}$ Polarization functions $(\zeta f=0.938)$ were added for $\mathrm{Ir}^{38}$ Geometric structures of all species were optimized at $\mathrm{T}=298.15 \mathrm{~K}$. Frequency calculations were performed to verify the optimized structures as local minima or transition states. Transition state structures were confirmed to connect appropriate reactants or products by intrinsic reaction coordinate (IRC) calculations. ${ }^{39}$

Table S5. Energies of calculated structures in transition states for hydride transfer.

\begin{tabular}{ccc|c}
\hline & TS $(\boldsymbol{R})$ & $\mathbf{T S}(\boldsymbol{S})$ & $\Delta(\mathrm{kcal} / \mathrm{mol})$ \\
\hline $\mathrm{E}$ & -5011.323619 & -5011.318153 & 3.4 \\
$\mathrm{G}$ & -5010.45865 & -5010.455229 & 2.1 \\
\hline
\end{tabular}

Coordinates for optimized structures:

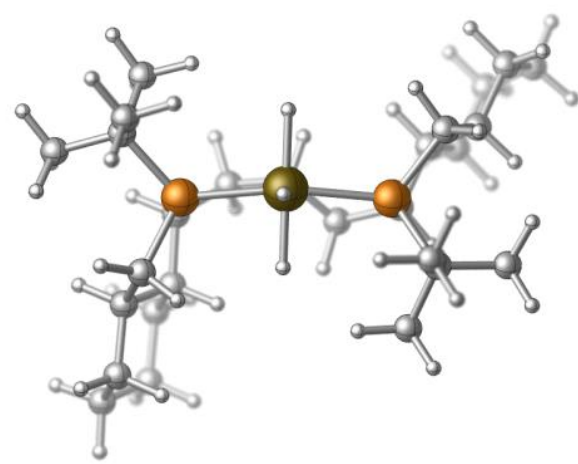

\begin{tabular}{lccc}
\multicolumn{4}{l}{ Heng-PNP/IrH } \\
P & -2.01248200 & 0.09302900 & 1.00791900 \\
$\mathrm{P}$ & 2.24627300 & -1.01035100 & 0.05429200 \\
$\mathrm{~N}$ & -0.18634500 & 0.11826000 & -1.35708200 \\
$\mathrm{H}$ & -0.79515900 & -0.56608500 & -1.79453400 \\
$\mathrm{C}$ & 1.03793200 & 0.18331400 & -2.20565300 \\
$\mathrm{H}$ & 1.00499400 & 1.09520200 & -2.81568300 \\
$\mathrm{H}$ & 0.98596000 & -0.66174800 & -2.89824800 \\
$\mathrm{C}$ & -0.94729100 & 1.38081300 & -1.25411700 \\
$\mathrm{H}$ & -1.12826900 & 1.80328100 & -2.25450100 \\
$\mathrm{H}$ & -0.32876400 & 2.08219500 & -0.68779500 \\
$\mathrm{C}$ & 2.36596600 & 0.10203000 & -1.45006600 \\
$\mathrm{H}$ & 3.11492400 & -0.28808700 & -2.15370300 \\
$\mathrm{C}$ & -3.33142700 & 0.37602100 & -1.35225000 \\
$\mathrm{H}$ & -2.84357500 & -0.48637200 & -1.83897400 \\
$\mathrm{C}$ & -6.09026900 & -0.27001500 & -2.17328700 \\
$\mathrm{H}$ & -6.67322300 & 0.53982700 & -1.71219700
\end{tabular}




\begin{tabular}{|c|c|c|c|}
\hline $\mathrm{H}$ & -6.80337200 & -0.90796500 & -2.70771900 \\
\hline $\mathrm{C}$ & 4.06717200 & 1.00272100 & 0.07389100 \\
\hline $\mathrm{H}$ & 4.84863900 & 0.55222600 & -0.56046700 \\
\hline $\mathrm{C}$ & 2.91650600 & 1.41267500 & -0.86397100 \\
\hline $\mathrm{H}$ & 2.12770900 & 1.86870700 & -0.24690600 \\
\hline $\mathrm{C}$ & -3.53716100 & -0.93320600 & 0.72577900 \\
\hline $\mathrm{H}$ & -3.18884900 & -1.90570500 & 0.36349100 \\
\hline $\mathrm{H}$ & -4.10702100 & -1.10697300 & 1.64371700 \\
\hline $\mathrm{C}$ & -2.27866400 & 1.14723600 & -0.53304200 \\
\hline $\mathrm{H}$ & -2.69739900 & 2.12417100 & -0.26077700 \\
\hline $\mathrm{C}$ & -4.34587400 & -0.20938400 & -0.35602900 \\
\hline $\mathrm{H}$ & -4.87088500 & 0.64040200 & 0.10591300 \\
\hline C & -5.38745100 & -1.07052500 & -1.07038400 \\
\hline $\mathrm{H}$ & -4.88711600 & -1.94559500 & -1.51033000 \\
\hline $\mathrm{H}$ & -6.12034000 & -1.45343400 & -0.34999400 \\
\hline $\mathrm{C}$ & -2.41711300 & 1.17943900 & 2.48618400 \\
\hline $\mathrm{C}$ & 3.53421000 & -0.08387600 & 1.02051800 \\
\hline $\mathrm{H}$ & 3.01737500 & 0.35996100 & 1.87466000 \\
\hline $\mathrm{H}$ & 4.32999200 & -0.72888200 & 1.40416200 \\
\hline $\mathrm{C}$ & 3.39214700 & 2.43841800 & -1.89250900 \\
\hline $\mathrm{H}$ & 2.56473200 & 2.75280200 & -2.54131300 \\
\hline $\mathrm{H}$ & 4.14697400 & 1.97260400 & -2.54400600 \\
\hline $\mathrm{C}$ & -4.03116300 & 1.18790200 & -2.44255000 \\
\hline $\mathrm{H}$ & -4.51490700 & 2.06093900 & -1.97993300 \\
\hline $\mathrm{H}$ & -3.30347700 & 1.57532200 & -3.16666100 \\
\hline $\mathrm{C}$ & -5.08546000 & 0.33563100 & -3.15982200 \\
\hline $\mathrm{H}$ & -4.58021000 & -0.47644200 & -3.70195700 \\
\hline $\mathrm{H}$ & -5.60920400 & 0.93825500 & -3.91067100 \\
\hline $\mathrm{C}$ & -3.85932100 & 1.70502200 & 2.45774600 \\
\hline $\mathrm{H}$ & -4.05334500 & 2.33058200 & 1.57893200 \\
\hline $\mathrm{H}$ & -4.03317700 & 2.32630700 & 3.34511900 \\
\hline $\mathrm{H}$ & -4.60003900 & 0.89930100 & 2.47491700 \\
\hline $\mathrm{C}$ & -2.20260900 & 0.31712700 & 3.73978900 \\
\hline $\mathrm{H}$ & -2.88128500 & -0.54407900 & 3.75994000 \\
\hline $\mathrm{H}$ & -2.39666500 & 0.91561900 & 4.63900100 \\
\hline $\mathrm{H}$ & -1.17632000 & -0.05989500 & 3.78272900 \\
\hline $\mathrm{C}$ & 3.01307600 & -2.65300700 & -0.41019800 \\
\hline C & 4.67186300 & 2.21691400 & 0.77767800 \\
\hline $\mathrm{H}$ & 5.50871100 & 1.90843000 & 1.41624200 \\
\hline $\mathrm{H}$ & 3.91174600 & 2.65602500 & 1.43981300 \\
\hline $\mathrm{C}$ & -1.45433100 & 2.37415100 & 2.51681500 \\
\hline $\mathrm{H}$ & -1.57329600 & 3.01407000 & 1.63357900 \\
\hline $\mathrm{H}$ & -0.41494200 & 2.04066000 & 2.55740400 \\
\hline $\mathrm{H}$ & -1.66710300 & 2.98744100 & 3.40164400 \\
\hline C & 2.90498200 & -3.55479100 & 0.82881800 \\
\hline $\mathrm{H}$ & 3.46373100 & -3.14218300 & 1.67748000 \\
\hline $\mathrm{H}$ & 3.31935000 & -4.54621100 & 0.60561100 \\
\hline $\mathrm{H}$ & 1.86028200 & -3.66991000 & 1.13447300 \\
\hline $\mathrm{C}$ & 4.47938600 & -2.52828800 & -0.84399800 \\
\hline $\mathrm{H}$ & 4.59921300 & -1.87015500 & -1.71195600 \\
\hline $\mathrm{H}$ & 4.86118200 & -3.51698100 & -1.12837800 \\
\hline $\mathrm{H}$ & 5.12015200 & -2.15067700 & -0.04061500 \\
\hline C & 4.00129600 & 3.66088700 & -1.19465500 \\
\hline $\mathrm{H}$ & 4.36679500 & 4.37705400 & -1.93984600 \\
\hline $\mathrm{H}$ & 3.21453100 & 4.17271500 & -0.62268300 \\
\hline $\mathrm{C}$ & 5.13496000 & 3.26311600 & -0.24267600 \\
\hline
\end{tabular}




$\begin{array}{cccc}\mathrm{H} & 5.52225000 & 4.14964100 & 0.27257200 \\ \mathrm{H} & 5.96797300 & 2.84911300 & -0.82849800 \\ \mathrm{C} & 2.18786400 & -3.25285800 & -1.55684200 \\ \mathrm{H} & 1.12524100 & -3.29082000 & -1.29630200 \\ \mathrm{H} & 2.53219000 & -4.27368400 & -1.76532900 \\ \mathrm{H} & 2.30277100 & -2.67251400 & -2.48122200 \\ \mathrm{H} & -0.47749500 & -2.24166300 & 0.16088100 \\ \mathrm{Ir} & 0.08265900 & -0.74367100 & 0.68290900 \\ \mathrm{H} & 0.26112500 & -1.37610600 & 2.13637600 \\ \mathrm{H} & 0.64432500 & 0.71921100 & 1.27396000\end{array}$

$\begin{array}{lrrr}\text { 2-bromobenzophenone } & & \\ \mathrm{C} & 0.33664500 & -1.05115400 & -0.95472100 \\ \mathrm{C} & -1.00685300 & -0.91400900 & -0.27883700 \\ \mathrm{C} & -1.59458900 & 0.30678300 & 0.05439700 \\ \mathrm{C} & -2.84698400 & 0.36276000 & 0.65769000 \\ \mathrm{H} & -3.28808300 & 1.32423000 & 0.89507300 \\ \mathrm{C} & -3.52467100 & -0.81840900 & 0.94162700 \\ \mathrm{H} & -4.49877400 & -0.77297800 & 1.41809300 \\ \mathrm{C} & -2.96081600 & -2.04617000 & 0.60582500 \\ \mathrm{H} & -3.49129100 & -2.96911000 & 0.81624600 \\ \mathrm{C} & -1.71555300 & -2.08792500 & -0.00933500 \\ \mathrm{H} & -1.27214800 & -3.03903400 & -0.28747900 \\ \mathrm{C} & 1.56571800 & -0.65679600 & -0.20752300 \\ \mathrm{C} & 1.54276200 & -0.38441600 & 1.16348200 \\ \mathrm{H} & 0.60755600 & -0.43439100 & 1.71281700 \\ \mathrm{C} & 2.71924200 & -0.05658300 & 1.82850100 \\ \mathrm{H} & 2.69813100 & 0.14910400 & 2.89416300 \\ \mathrm{C} & 3.92056000 & 0.00803000 & 1.12650100 \\ \mathrm{H} & 4.83811400 & 0.26874400 & 1.64572300 \\ \mathrm{C} & 3.94864500 & -0.26325700 & -0.24158500 \\ \mathrm{H} & 4.88600300 & -0.21236500 & -0.78690600 \\ \mathrm{C} & 2.77665500 & -0.60054700 & -0.90503200 \\ \mathrm{H} & 2.77736400 & -0.82094000 & -1.96751400 \\ \mathrm{O} & 0.39426100 & -1.54027100 & -2.06740500 \\ \mathrm{Br} & -0.72501500 & 1.94397300 & -0.35676900\end{array}$

TS $(R)$

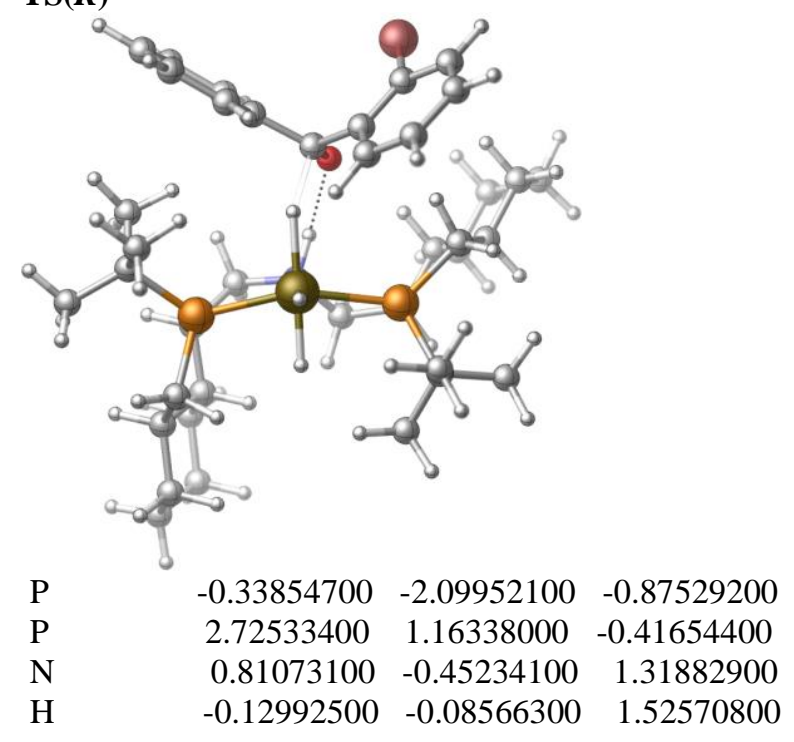




\begin{tabular}{|c|c|c|c|}
\hline C & 1.76775500 & 0.38067600 & 2.09237400 \\
\hline $\mathrm{H}$ & 1.97400800 & -0.09556200 & 3.06037200 \\
\hline $\mathrm{H}$ & 1.25529700 & 1.32498400 & 2.29986600 \\
\hline $\mathrm{C}$ & 0.83625000 & -1.88849600 & 1.67511400 \\
\hline $\mathrm{H}$ & 0.68116200 & -2.00665700 & 2.75773300 \\
\hline $\mathrm{H}$ & 1.83189100 & -2.27174300 & 1.43854700 \\
\hline $\mathrm{C}$ & 3.07429900 & 0.69139200 & 1.35961200 \\
\hline $\mathrm{H}$ & 3.56212100 & 1.52267100 & 1.88786800 \\
\hline $\mathrm{C}$ & -1.66232300 & -2.53045500 & 1.44518400 \\
\hline $\mathrm{H}$ & -1.86464700 & -1.46132400 & 1.59955400 \\
\hline $\mathrm{C}$ & -4.37795100 & -3.50073900 & 2.04312900 \\
\hline $\mathrm{H}$ & -4.28933600 & -4.58647900 & 1.89235100 \\
\hline $\mathrm{H}$ & -5.41401700 & -3.30610600 & 2.34345900 \\
\hline $\mathrm{C}$ & 5.12591600 & 0.04177600 & 0.17428400 \\
\hline $\mathrm{H}$ & 5.63325800 & 0.92206200 & 0.60127100 \\
\hline $\mathrm{C}$ & 4.10428300 & -0.44109200 & 1.21973500 \\
\hline $\mathrm{H}$ & 3.60244000 & -1.32064900 & 0.79069000 \\
\hline $\mathrm{C}$ & -2.18769900 & -2.23877000 & -0.93739300 \\
\hline $\mathrm{H}$ & -2.59254200 & -1.22928600 & -0.90341400 \\
\hline $\mathrm{H}$ & -2.54335100 & -2.69885800 & -1.86391800 \\
\hline $\mathrm{C}$ & -0.22554500 & -2.67932400 & 0.90971200 \\
\hline $\mathrm{H}$ & 0.05229800 & -3.74128900 & 0.93960600 \\
\hline $\mathrm{C}$ & -2.60790400 & -2.99539200 & 0.32693000 \\
\hline $\mathrm{H}$ & -2.44528400 & -4.07389600 & 0.17629600 \\
\hline $\mathrm{C}$ & -4.06838200 & -2.78219100 & 0.72482700 \\
\hline $\mathrm{H}$ & -4.24857100 & -1.70510400 & 0.84396500 \\
\hline $\mathrm{H}$ & -4.73698700 & -3.13383600 & -0.07077800 \\
\hline C & 0.26778400 & -3.51850000 & -1.95621700 \\
\hline $\mathrm{C}$ & 4.33565700 & 0.49294400 & -1.06567500 \\
\hline $\mathrm{H}$ & 4.09258300 & -0.36004600 & -1.70358300 \\
\hline $\mathrm{H}$ & 4.88903000 & 1.21511900 & -1.67105100 \\
\hline C & 4.78916600 & -0.86015200 & 2.51902800 \\
\hline $\mathrm{H}$ & 4.05189000 & -1.23687600 & 3.23932500 \\
\hline $\mathrm{H}$ & 5.26059200 & 0.02143700 & 2.97854100 \\
\hline $\mathrm{C}$ & -1.95199700 & -3.27096700 & 2.74966300 \\
\hline $\mathrm{H}$ & -1.75091000 & -4.34455400 & 2.61141100 \\
\hline $\mathrm{H}$ & -1.28907500 & -2.92298100 & 3.55170000 \\
\hline C & -3.41724300 & -3.06799900 & 3.15671200 \\
\hline $\mathrm{H}$ & -3.57992600 & -2.00345200 & 3.37379400 \\
\hline $\mathrm{H}$ & -3.63373700 & -3.61957900 & 4.07923800 \\
\hline $\mathrm{C}$ & -0.56126000 & -4.79628100 & -1.75148300 \\
\hline $\mathrm{H}$ & -0.48305800 & -5.17908500 & -0.72784000 \\
\hline $\mathrm{H}$ & -0.18337000 & -5.57744900 & -2.42249600 \\
\hline $\mathrm{H}$ & -1.62053300 & -4.65371200 & -1.98307700 \\
\hline C & 0.14435600 & -3.05093800 & -3.41460700 \\
\hline $\mathrm{H}$ & -0.89763800 & -2.83760500 & -3.68217800 \\
\hline $\mathrm{H}$ & 0.50772800 & -3.83631300 & -4.08946200 \\
\hline $\mathrm{H}$ & 0.73112300 & -2.14291200 & -3.58305100 \\
\hline $\mathrm{C}$ & 2.84646000 & 3.03002500 & -0.52088100 \\
\hline $\mathrm{C}$ & 6.18352600 & -1.02706300 & -0.09894700 \\
\hline $\mathrm{H}$ & 6.92312700 & -0.65627300 & -0.81900100 \\
\hline $\mathrm{H}$ & 5.69609600 & -1.89713700 & -0.56285300 \\
\hline C & 1.73500300 & -3.84630300 & -1.64410000 \\
\hline $\mathrm{H}$ & 1.88341000 & -4.08784200 & -0.58446400 \\
\hline $\mathrm{H}$ & 2.39517100 & -3.01435700 & -1.89728200 \\
\hline $\mathrm{H}$ & 2.04103400 & -4.72201800 & -2.23036500 \\
\hline
\end{tabular}




\begin{tabular}{lrrr}
$\mathrm{C}$ & 2.29356700 & 3.44165600 & -1.89246500 \\
$\mathrm{H}$ & 2.86952000 & 2.99333600 & -2.71116900 \\
$\mathrm{H}$ & 2.34267100 & 4.53260900 & -2.00124100 \\
$\mathrm{H}$ & 1.25084700 & 3.12800700 & -2.00090400 \\
$\mathrm{C}$ & 4.27878700 & 3.55157900 & -0.34724800 \\
$\mathrm{H}$ & 4.73979000 & 3.18714300 & 0.57835500 \\
$\mathrm{H}$ & 4.25679400 & 4.64719600 & -0.29267400 \\
$\mathrm{H}$ & 4.92947600 & 3.27989600 & -1.18401700 \\
$\mathrm{C}$ & 5.85509000 & -1.92882300 & 2.24852400 \\
$\mathrm{H}$ & 6.36709100 & -2.19461300 & 3.18044600 \\
$\mathrm{H}$ & 5.36238100 & -2.84195300 & 1.88563300 \\
$\mathrm{C}$ & 6.87140100 & -1.45747500 & 1.20206600 \\
$\mathrm{H}$ & 7.60000000 & -2.25076900 & 0.99916900 \\
$\mathrm{H}$ & 7.43580900 & -0.60612400 & 1.60816700 \\
$\mathrm{C}$ & 1.96692000 & 3.61276600 & 0.59010600 \\
$\mathrm{H}$ & 0.97328600 & 3.16258600 & 0.58904200 \\
$\mathrm{H}$ & 1.83912500 & 4.69072800 & 0.44008600 \\
$\mathrm{H}$ & 2.41594400 & 3.46683200 & 1.57949000 \\
$\mathrm{H}$ & -0.39206900 & 1.01681800 & -0.64545700 \\
$\mathrm{Ir}$ & 0.90124500 & -0.15176000 & -0.85699900 \\
$\mathrm{H}$ & 0.91530100 & 0.08377500 & -2.44575200 \\
$\mathrm{C}$ & -1.00832100 & 5.64475900 & 0.71346000 \\
$\mathrm{C}$ & -1.10217200 & 5.10987000 & -0.57112600 \\
$\mathrm{C}$ & -1.33626000 & 3.75133200 & -0.74633000 \\
$\mathrm{C}$ & -1.46248100 & 2.90963900 & 0.36092700 \\
$\mathrm{C}$ & -1.37139600 & 3.44938700 & 1.64367600 \\
$\mathrm{C}$ & -1.14504500 & 4.81160100 & 1.82058700 \\
$\mathrm{H}$ & -0.83236800 & 6.70795700 & 0.84938300 \\
$\mathrm{H}$ & -0.99552100 & 5.75465100 & -1.43863500 \\
$\mathrm{H}$ & -1.07957500 & 5.22433200 & 2.82325700 \\
$\mathrm{C}$ & -1.76497700 & 1.42931000 & 0.24325800 \\
$\mathrm{O}$ & -1.75198100 & 0.73710600 & 1.28283500 \\
$\mathrm{C}$ & -2.78150800 & 1.10481000 & -0.85499400 \\
$\mathrm{C}$ & -2.42887100 & 0.93174900 & -2.19788700 \\
$\mathrm{C}$ & -4.13692000 & 0.98331100 & -0.52936700 \\
$\mathrm{C}$ & -3.37290400 & 0.61902500 & -3.16689000 \\
$\mathrm{H}$ & -1.37662100 & 0.98656600 & -2.45656900 \\
$\mathrm{C}$ & -5.09177600 & 0.65520700 & -1.48971200 \\
$\mathrm{C}$ & -4.70947600 & 0.46567000 & -2.81159700 \\
$\mathrm{H}$ & -3.05753500 & 0.47628800 & -4.19593200 \\
$\mathrm{H}$ & -6.13033400 & 0.55169000 & -1.19571100 \\
$\mathrm{H}$ & -5.45556700 & 0.20283600 & -3.55500300 \\
$\mathrm{H}$ & 2.09537500 & -1.21962700 & -1.07680400 \\
$\mathrm{H}$ & -1.48725600 & 2.78196500 & 2.49122100 \\
$\mathrm{H}$ & -1.40933100 & 3.34021000 & -1.74800600 \\
$\mathrm{Br}$ & -4.78909700 & 1.26663100 & 1.23620500 \\
& & & \\
\hline
\end{tabular}

TS(S) 


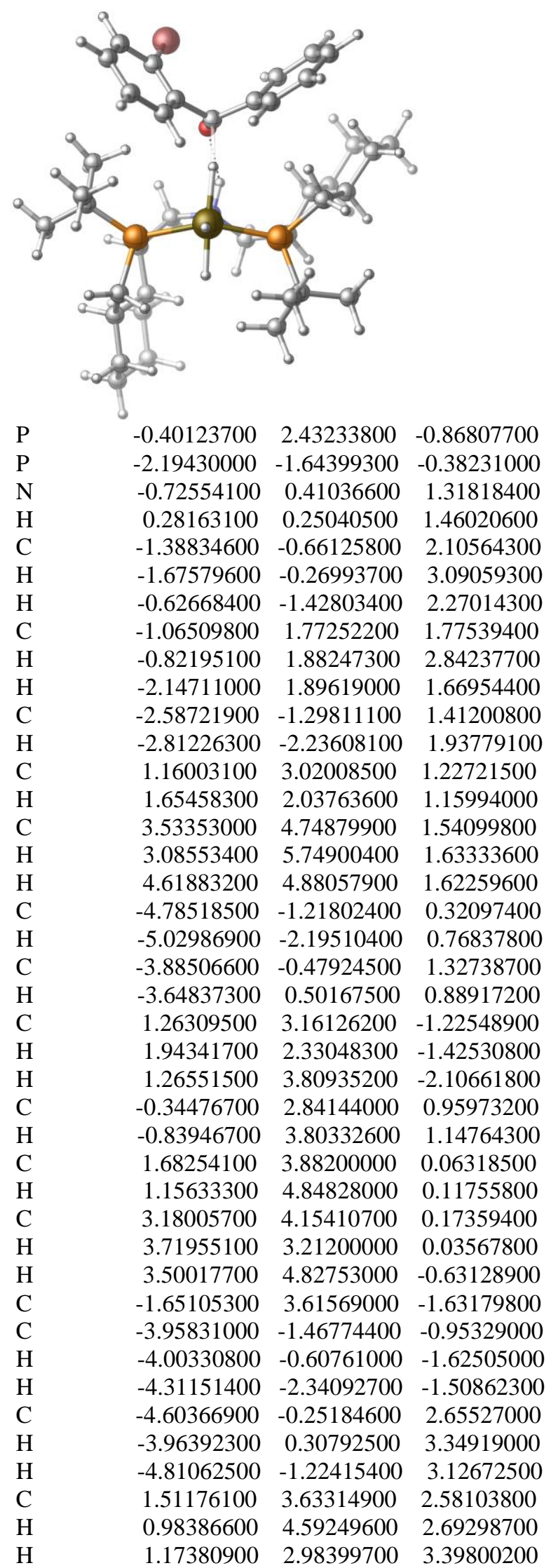




\begin{tabular}{|c|c|c|c|}
\hline C & 3.02369400 & 3.86595900 & 2.68512500 \\
\hline $\mathrm{H}$ & 3.53671300 & 2.89510700 & 2.64674900 \\
\hline $\mathrm{H}$ & 3.26838000 & 4.31965000 & 3.65250300 \\
\hline $\mathrm{C}$ & -1.21218000 & 5.08150100 & -1.48811400 \\
\hline $\mathrm{H}$ & -1.11088700 & 5.37968700 & -0.43891700 \\
\hline $\mathrm{H}$ & -1.97320100 & 5.72931200 & -1.94002900 \\
\hline $\mathrm{H}$ & -0.26547400 & 5.28985800 & -1.99478800 \\
\hline $\mathrm{C}$ & -1.77339100 & 3.25334900 & -3.11906300 \\
\hline $\mathrm{H}$ & -0.81480900 & 3.36517500 & -3.63993100 \\
\hline $\mathrm{H}$ & -2.49817100 & 3.91832700 & -3.60559900 \\
\hline $\mathrm{H}$ & -2.10549000 & 2.21810800 & -3.24136400 \\
\hline $\mathrm{C}$ & -1.82866200 & -3.47735600 & -0.48861500 \\
\hline C & -6.09421200 & -0.46340500 & 0.09157800 \\
\hline $\mathrm{H}$ & -6.73916000 & -1.02039200 & -0.59887200 \\
\hline $\mathrm{H}$ & -5.86792700 & 0.49968200 & -0.38943900 \\
\hline $\mathrm{C}$ & -3.01631500 & 3.45713700 & -0.94574700 \\
\hline $\mathrm{H}$ & -2.96449200 & 3.69453900 & 0.12367100 \\
\hline $\mathrm{H}$ & -3.40947600 & 2.44335200 & -1.05351300 \\
\hline $\mathrm{H}$ & -3.73135800 & 4.15262200 & -1.40262300 \\
\hline $\mathrm{C}$ & -1.50133700 & -3.77436400 & -1.95829800 \\
\hline $\mathrm{H}$ & -2.38088500 & -3.62413900 & -2.59631800 \\
\hline $\mathrm{H}$ & -1.17627800 & -4.81639500 & -2.06959500 \\
\hline $\mathrm{H}$ & -0.70335400 & -3.12636900 & -2.32543900 \\
\hline $\mathrm{C}$ & -3.00266100 & -4.36084900 & -0.04260700 \\
\hline $\mathrm{H}$ & -3.32255100 & -4.14031400 & 0.98171800 \\
\hline $\mathrm{H}$ & -2.68674500 & -5.41159700 & -0.06625800 \\
\hline $\mathrm{H}$ & -3.87301100 & -4.26817300 & -0.69993100 \\
\hline C & -5.91987900 & 0.50286100 & 2.43067100 \\
\hline $\mathrm{H}$ & -6.44760100 & 0.63220300 & 3.38255700 \\
\hline $\mathrm{H}$ & -5.69306500 & 1.51086400 & 2.05482100 \\
\hline $\mathrm{C}$ & -6.81957100 & -0.21701700 & 1.41989300 \\
\hline $\mathrm{H}$ & -7.73406900 & 0.36240800 & 1.24856200 \\
\hline $\mathrm{H}$ & -7.13275900 & -1.18245500 & 1.84210000 \\
\hline $\mathrm{C}$ & -0.62579100 & -3.75270200 & 0.42536300 \\
\hline $\mathrm{H}$ & 0.14650000 & -2.98811500 & 0.33194100 \\
\hline $\mathrm{H}$ & -0.17104800 & -4.71962500 & 0.17830600 \\
\hline $\mathrm{H}$ & -0.93505700 & -3.78831700 & 1.47671800 \\
\hline $\mathrm{H}$ & 0.80863600 & -0.34979500 & -0.79792800 \\
\hline Ir & -0.87136600 & 0.19751900 & -0.87637500 \\
\hline $\mathrm{H}$ & -0.88327900 & 0.02171000 & -2.47203000 \\
\hline $\mathrm{C}$ & 2.68013800 & -4.73285100 & -1.80266400 \\
\hline $\mathrm{C}$ & 1.99671100 & -3.71393300 & -2.45837100 \\
\hline $\mathrm{C}$ & 1.79351900 & -2.49609900 & -1.82456300 \\
\hline C & 2.26418400 & -2.24885300 & -0.53220200 \\
\hline C & 2.97116900 & -3.27924400 & 0.09753200 \\
\hline C & 3.16973800 & -4.51196500 & -0.52227700 \\
\hline $\mathrm{H}$ & 2.83528200 & -5.69522300 & -2.28049700 \\
\hline $\mathrm{H}$ & 1.60064600 & -3.87174600 & -3.45691300 \\
\hline $\mathrm{H}$ & 1.20984500 & -1.71816100 & -2.30665300 \\
\hline $\mathrm{H}$ & 3.71240300 & -5.29044900 & 0.00252900 \\
\hline C & 2.11264200 & -0.88336700 & 0.14993200 \\
\hline $\mathrm{O}$ & 1.74357200 & -0.81808000 & 1.33905200 \\
\hline C & 3.19596500 & 0.06874200 & -0.33233800 \\
\hline $\mathrm{C}$ & 3.41985800 & 0.38227800 & -1.67473000 \\
\hline $\mathrm{C}$ & 4.11474200 & 0.49458700 & 0.62832400 \\
\hline $\mathrm{C}$ & 4.54729900 & 1.10513100 & -2.04860300 \\
\hline
\end{tabular}




$\begin{array}{lrrr}\mathrm{H} & 2.69951600 & 0.07097300 & -2.42480300 \\ \mathrm{C} & 5.25595800 & 1.19980900 & 0.25194900 \\ \mathrm{H} & 3.93245200 & 0.23994400 & 1.66708400 \\ \mathrm{C} & 5.47736100 & 1.50308500 & -1.08815200 \\ \mathrm{H} & 4.70644000 & 1.34999400 & -3.09466700 \\ \mathrm{H} & 5.97045200 & 1.51331300 & 1.00765200 \\ \mathrm{H} & 6.36583200 & 2.05286100 & -1.38462900 \\ \mathrm{Br} & 3.74050000 & -3.08918500 & 1.82817500 \\ \mathrm{H} & -2.37200600 & 0.76793800 & -1.03558800\end{array}$


8. NMR spectra of all the compounds and complexes.

${ }^{1} \mathrm{H}$ NMR (400 MHz, $\left.\mathrm{CDCl}_{3}\right)$ of 3

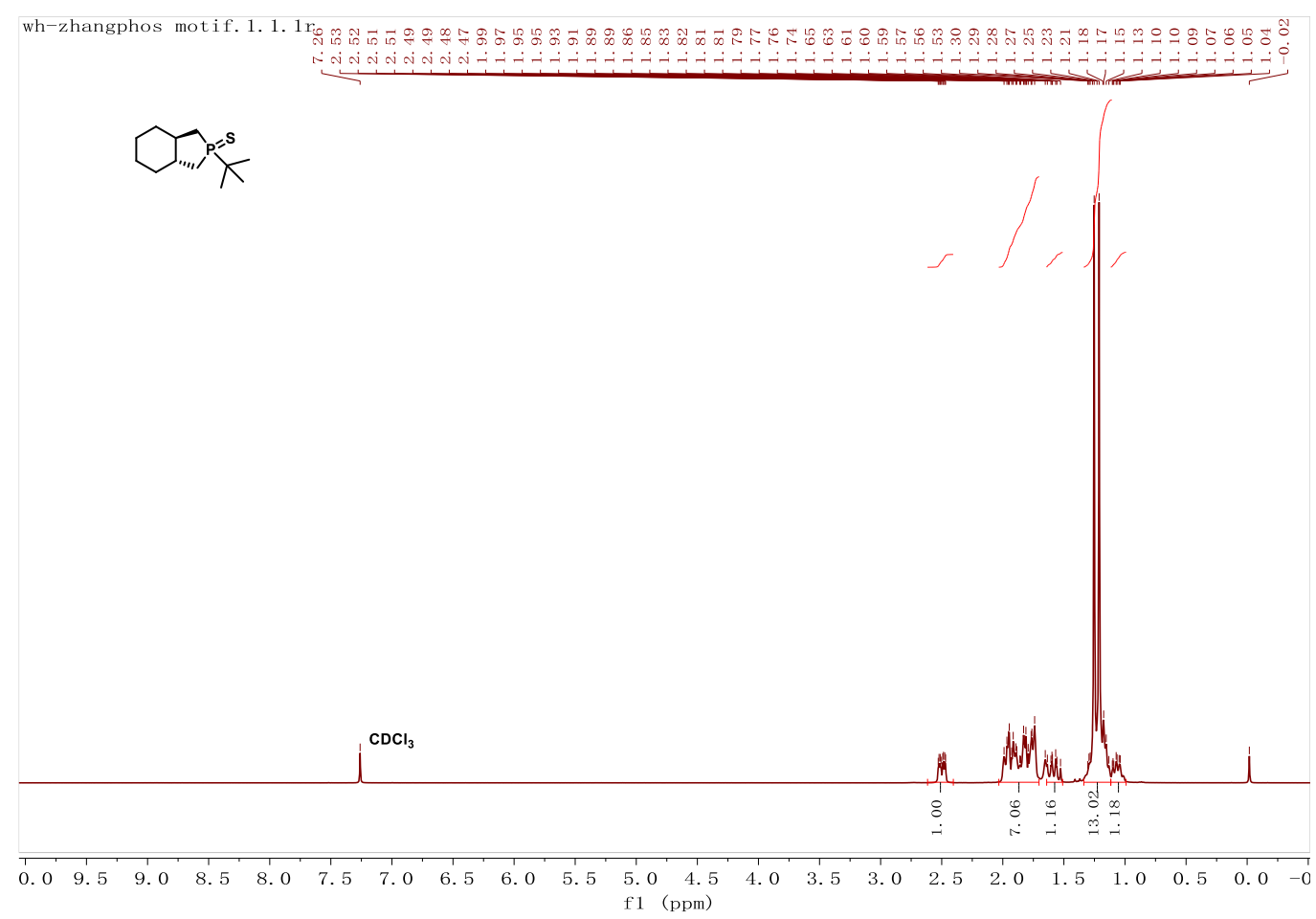

${ }^{31} \mathrm{P}$ NMR (162 MHz, $\left.\mathrm{CDCl}_{3}\right)$ of 3

wh-zhangphos motif. 2 1. $1 \mathrm{r}$
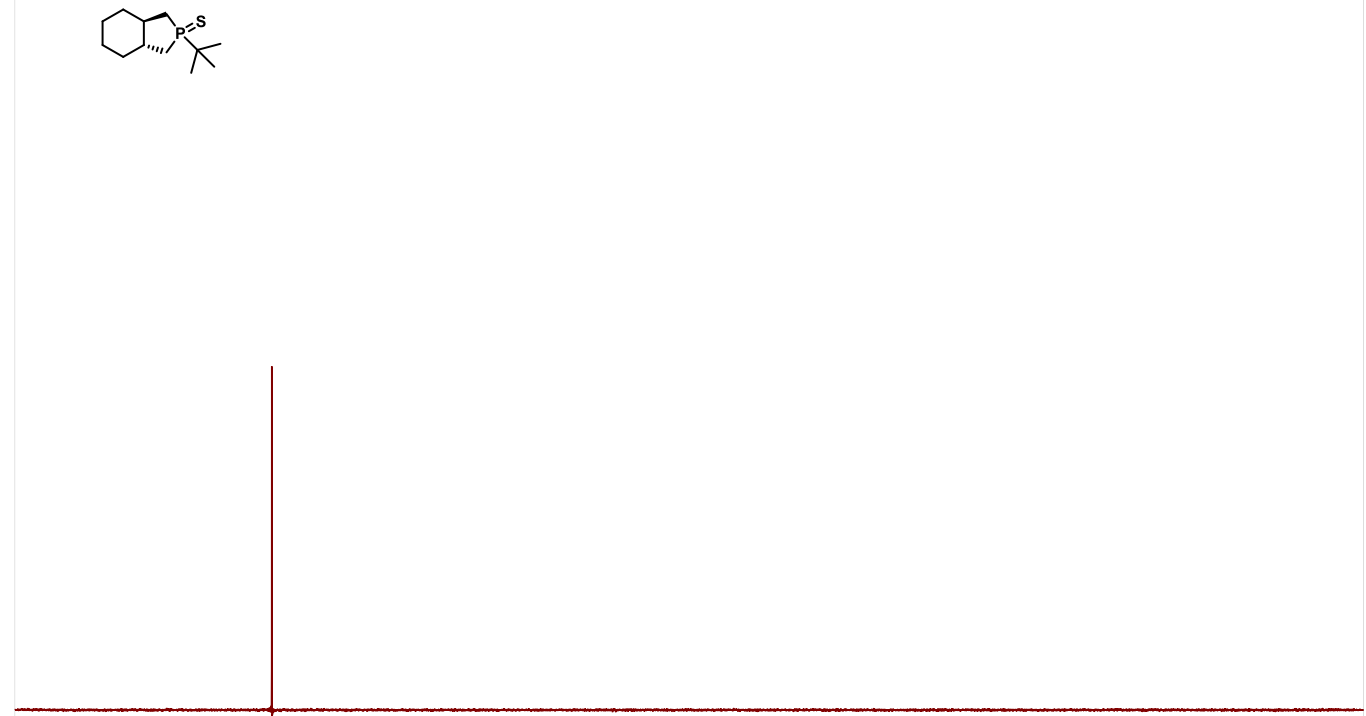

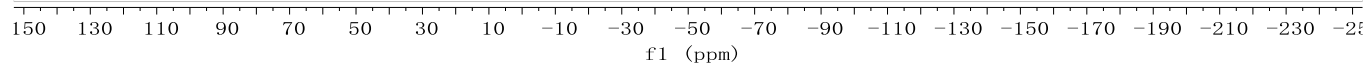


${ }^{1} \mathrm{H}$ NMR (400 MHz, $\left.\mathrm{CDCl}_{3}\right)$ of 4

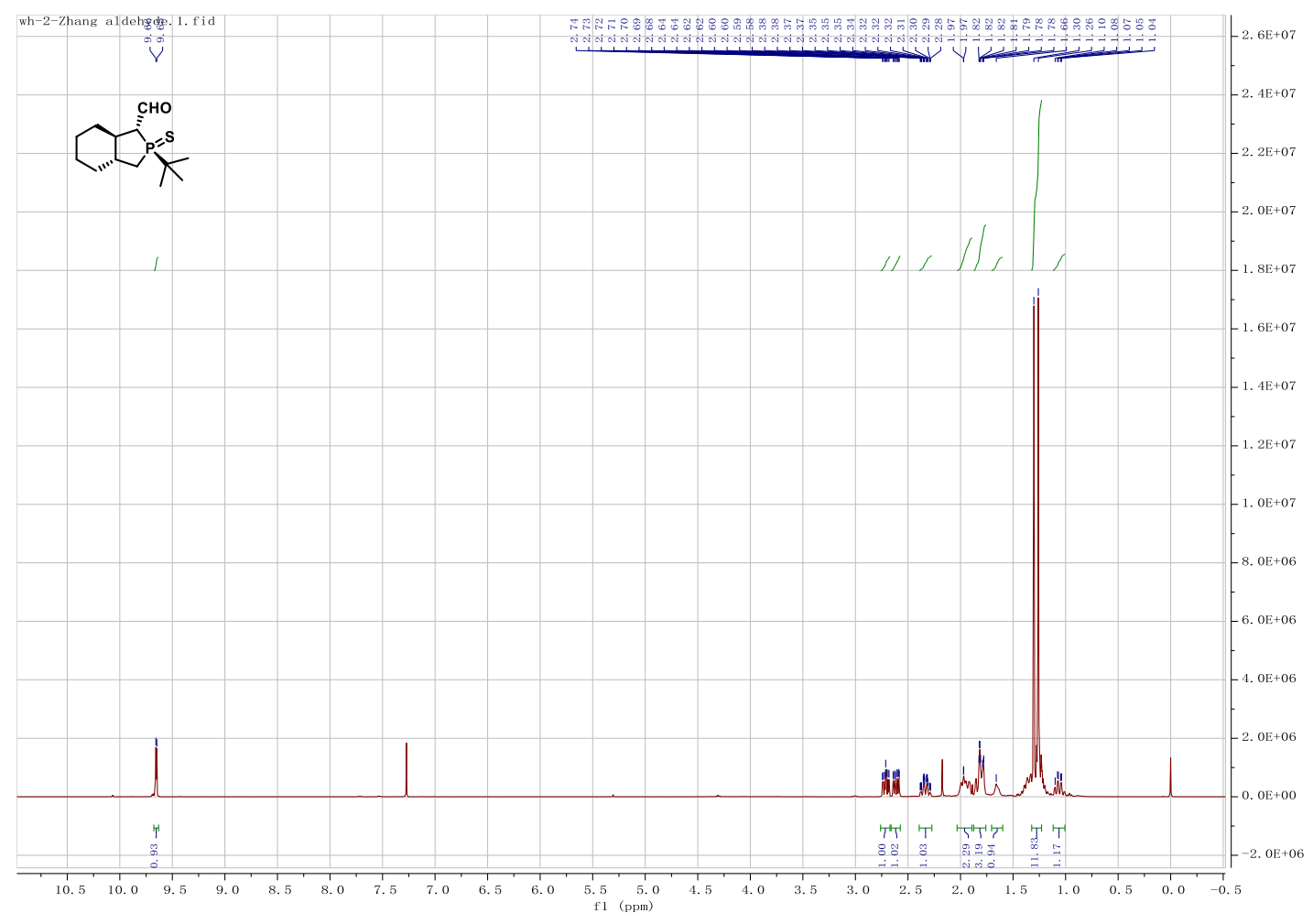

${ }^{13} \mathrm{C}$ NMR (101 MHz, $\left.\mathrm{CDCl}_{3}\right)$ of 4

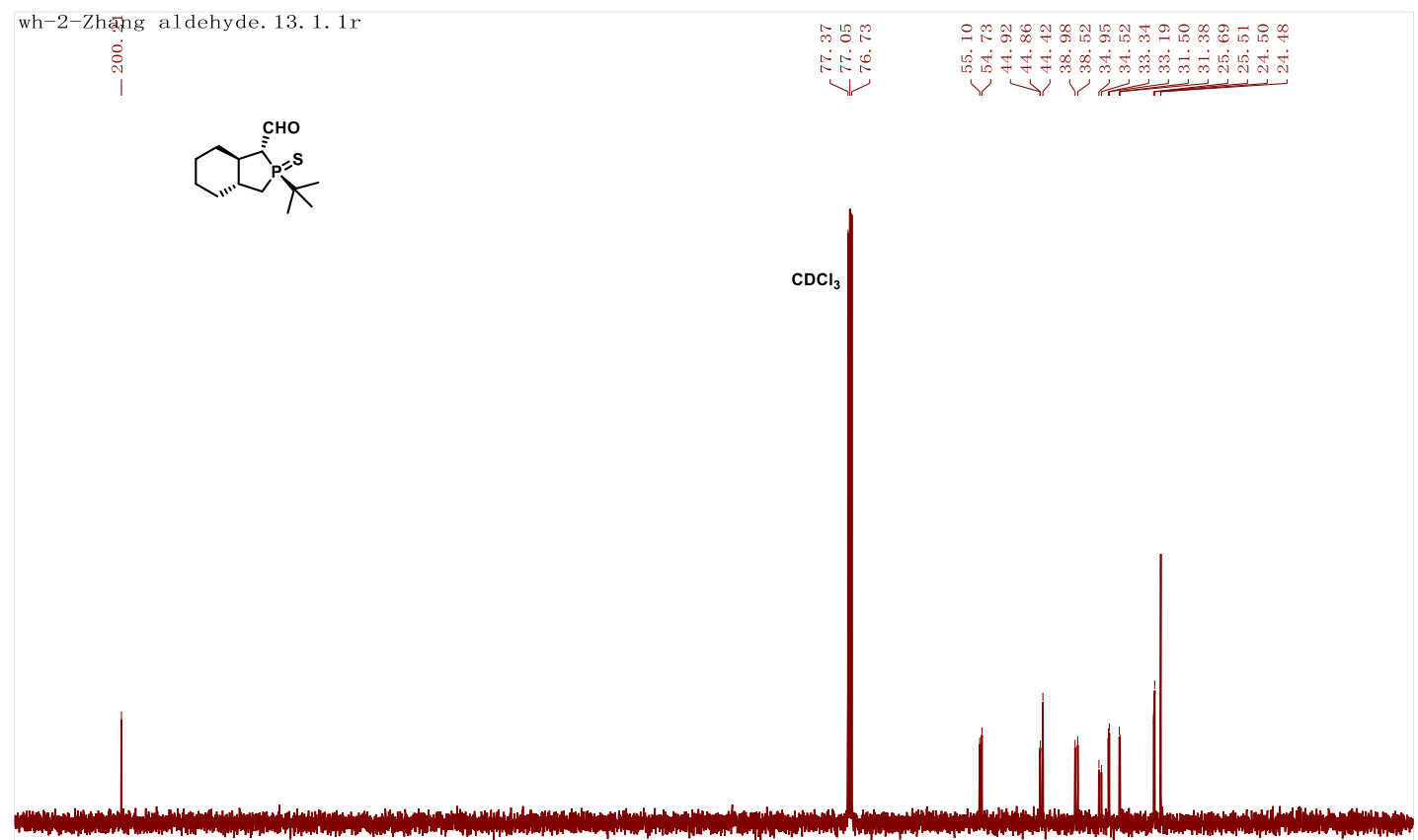

$\begin{array}{lllllllllllllllllllllll}210 & 200 & 190 & 180 & 170 & 160 & 150 & 140 & 130 & 120 & 110 & 100 & 90 & 80 & 70 & 60 & 50 & 40 & 30 & 20 & 10 & 0 & -10\end{array}$ f1 (ppm) 
${ }^{31} \mathrm{P}$ NMR (162 MHz, $\left.\mathrm{CDCl}_{3}\right)$ of 4

wh-2-Zhang aldehyde. $31,1,1 \mathrm{r}$<smiles>CC(C)(C)P1(=S)C[C@@H]2CCCCC2[C@@H]1CO</smiles>

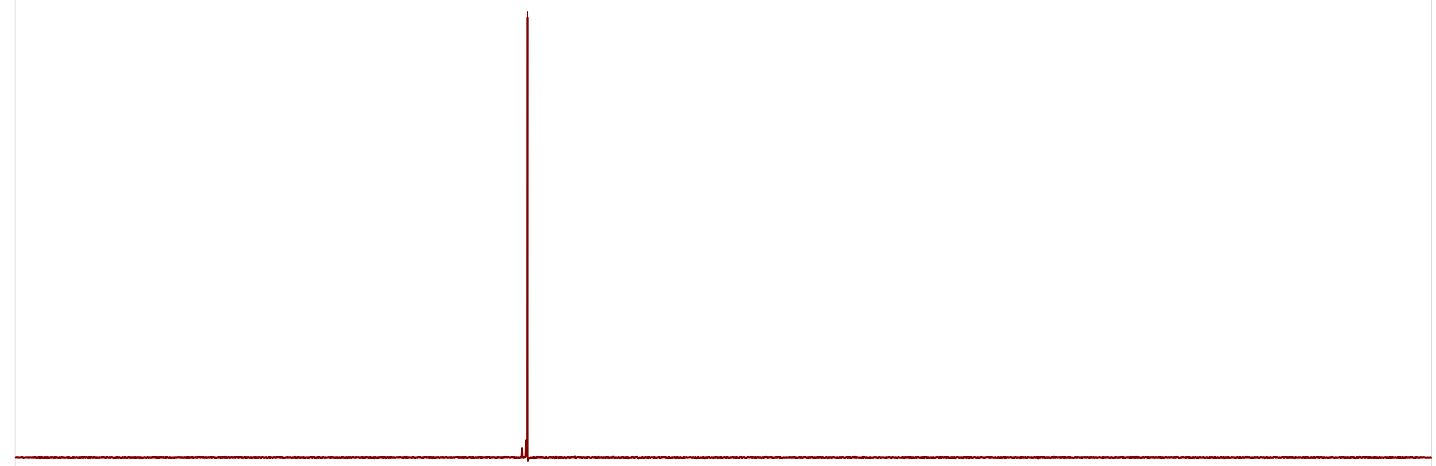

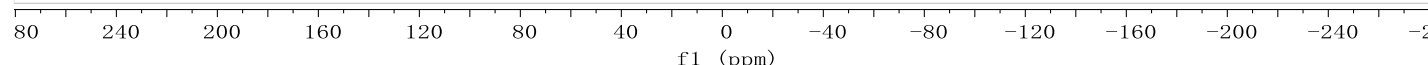


${ }^{1} \mathrm{H}$ NMR (400 MHz, $\left.\mathrm{CDCl}_{3}\right)$ of 5

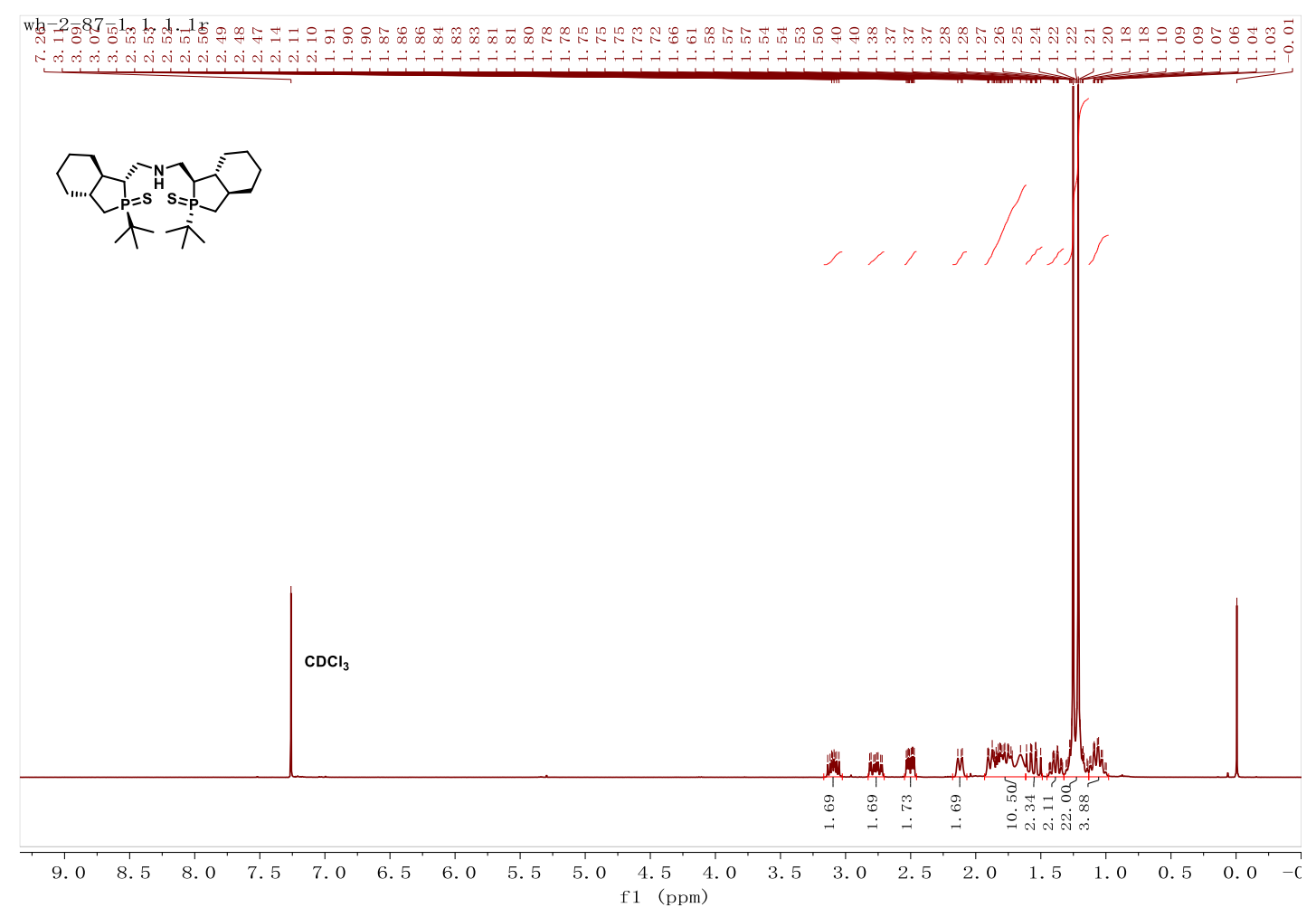

${ }^{13} \mathrm{C}$ NMR (101 MHz, $\left.\mathrm{CDCl}_{3}\right)$ of 5

wh-2-87-1. 13. 1. $1 \mathrm{r}$
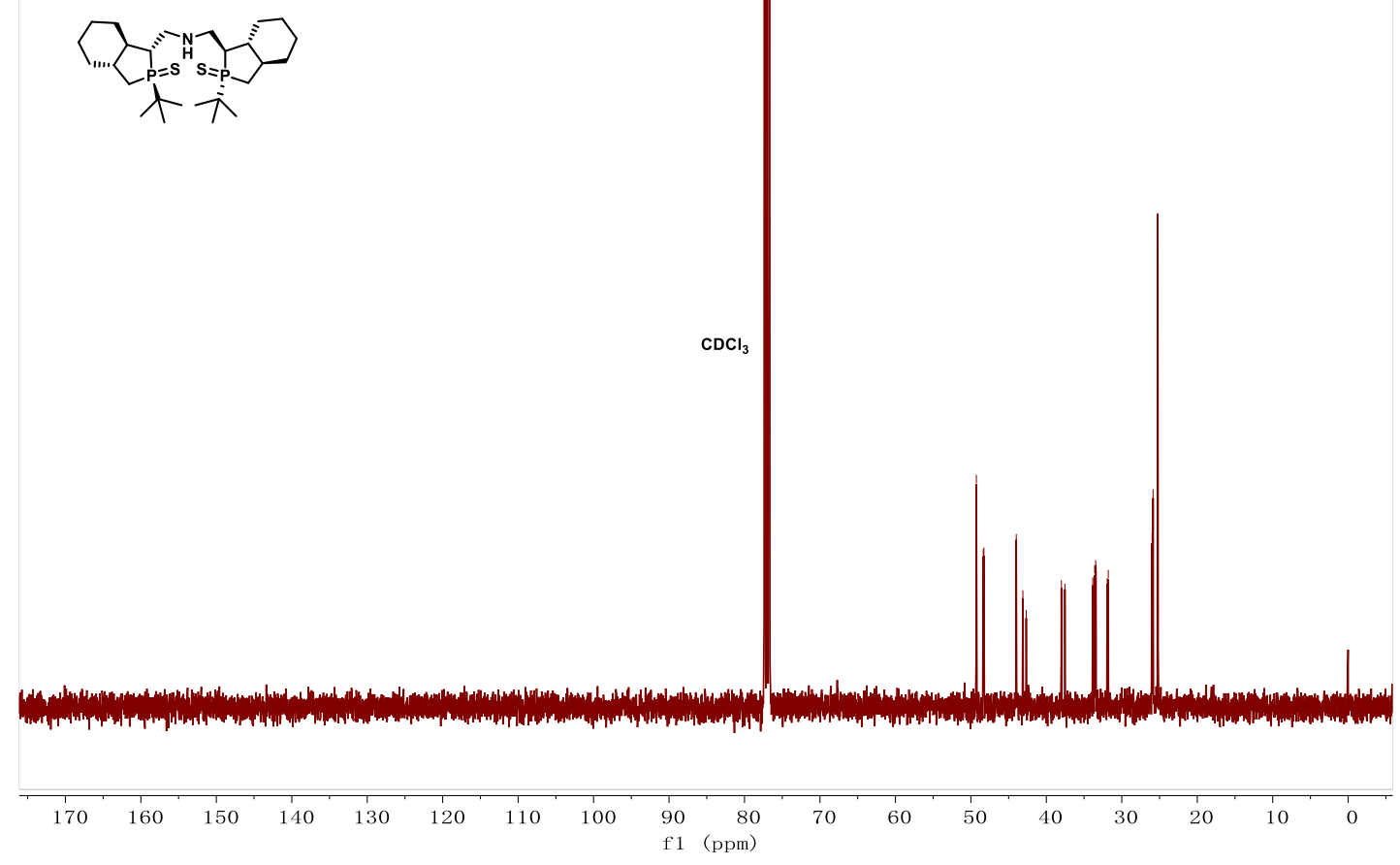
${ }^{31} \mathrm{P}$ NMR (162 MHz, $\left.\mathrm{CDCl}_{3}\right)$ of 5

wh-2-87-1. 14. 1. 1

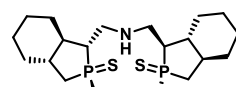

$\lambda+$

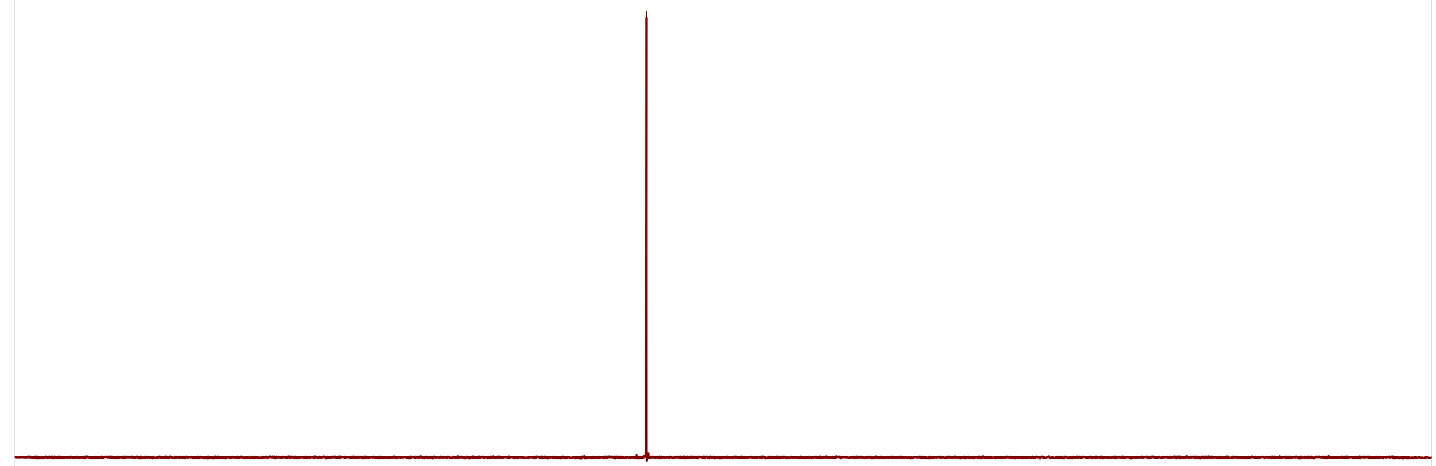

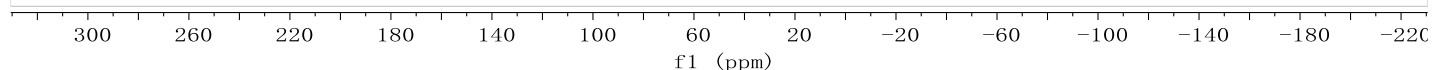


${ }^{1} \mathrm{H}$ NMR (400 MHz, $\left.\mathrm{C}_{6} \mathrm{D}_{6}\right)$ of Heng-PNP

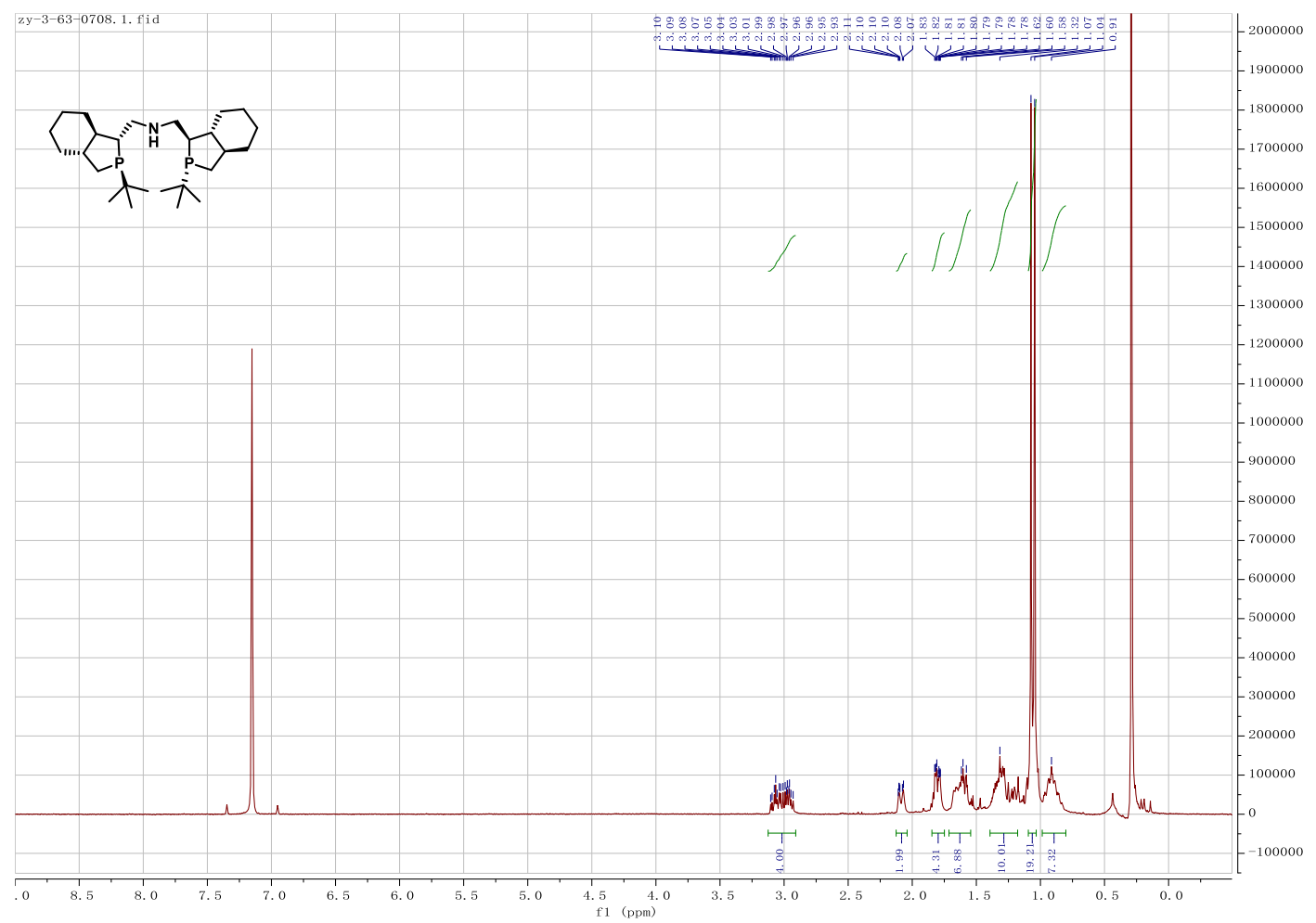

${ }^{13} \mathrm{C}$ NMR (101 MHz, $\left.\mathrm{C}_{6} \mathrm{D}_{6}\right)$ of Heng-PNP

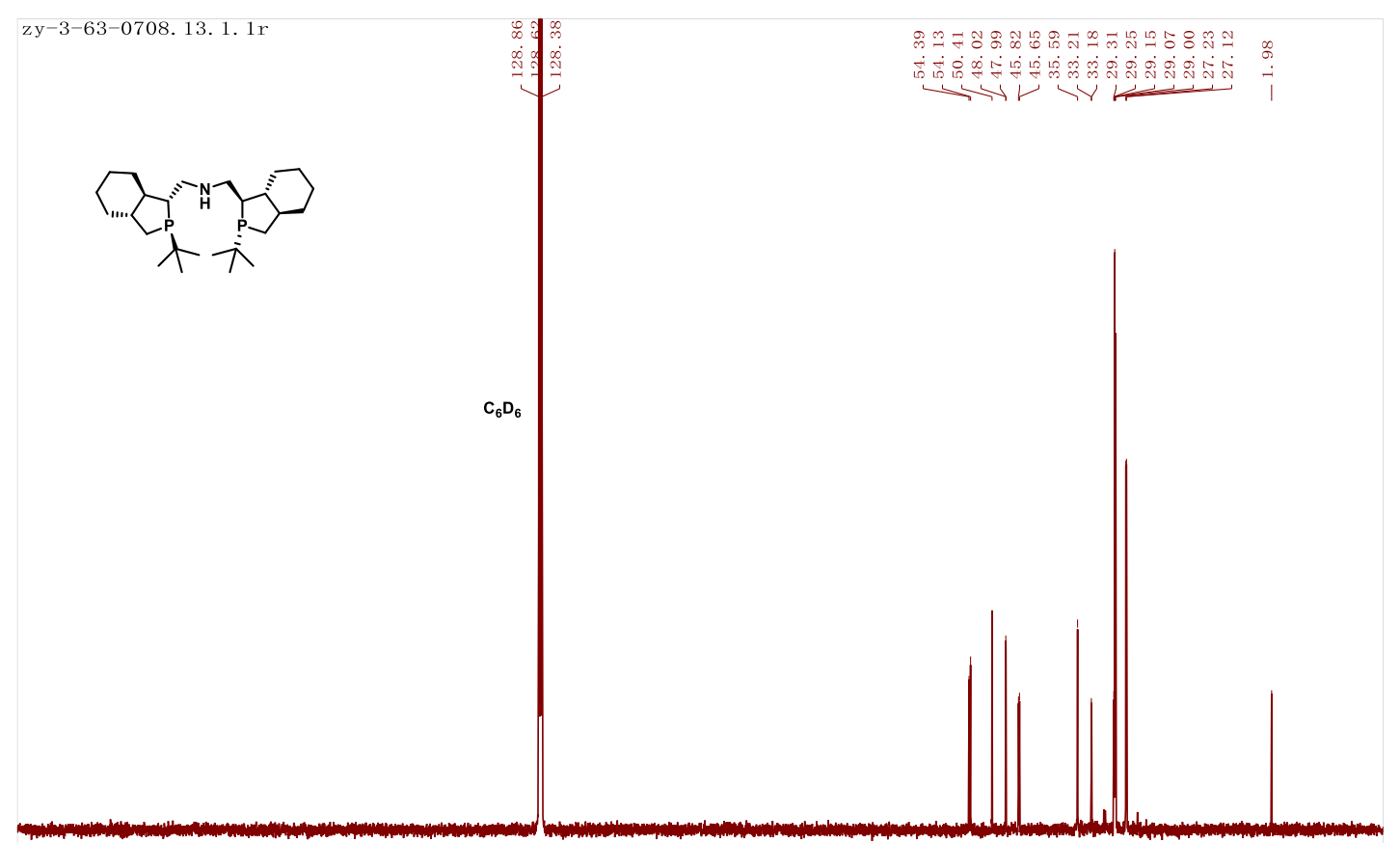

$\begin{array}{lllllllllllllllllllllllllllll}210 & 200 & 190 & 180 & 170 & 160 & 150 & 140 & 130 & 120 & 110 & 100 & 90 & 80 & 70 & 60 & 50 & 40 & 30 & 20 & 10 & 0 & -10\end{array}$ 
${ }^{31}$ P NMR (162 MHz, $\left.\mathrm{C}_{6} \mathrm{D}_{6}\right)$ of Heng-PNP

zy-3-63-0708.31.1.1r

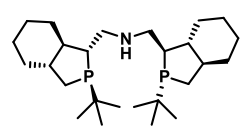

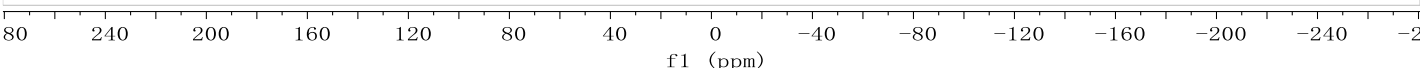


${ }^{1} \mathrm{H}$ NMR (600 MHz, $\left.\mathrm{C}_{6} \mathrm{D}_{6}\right)$ of crude Ir/Heng-PNP precatalyst

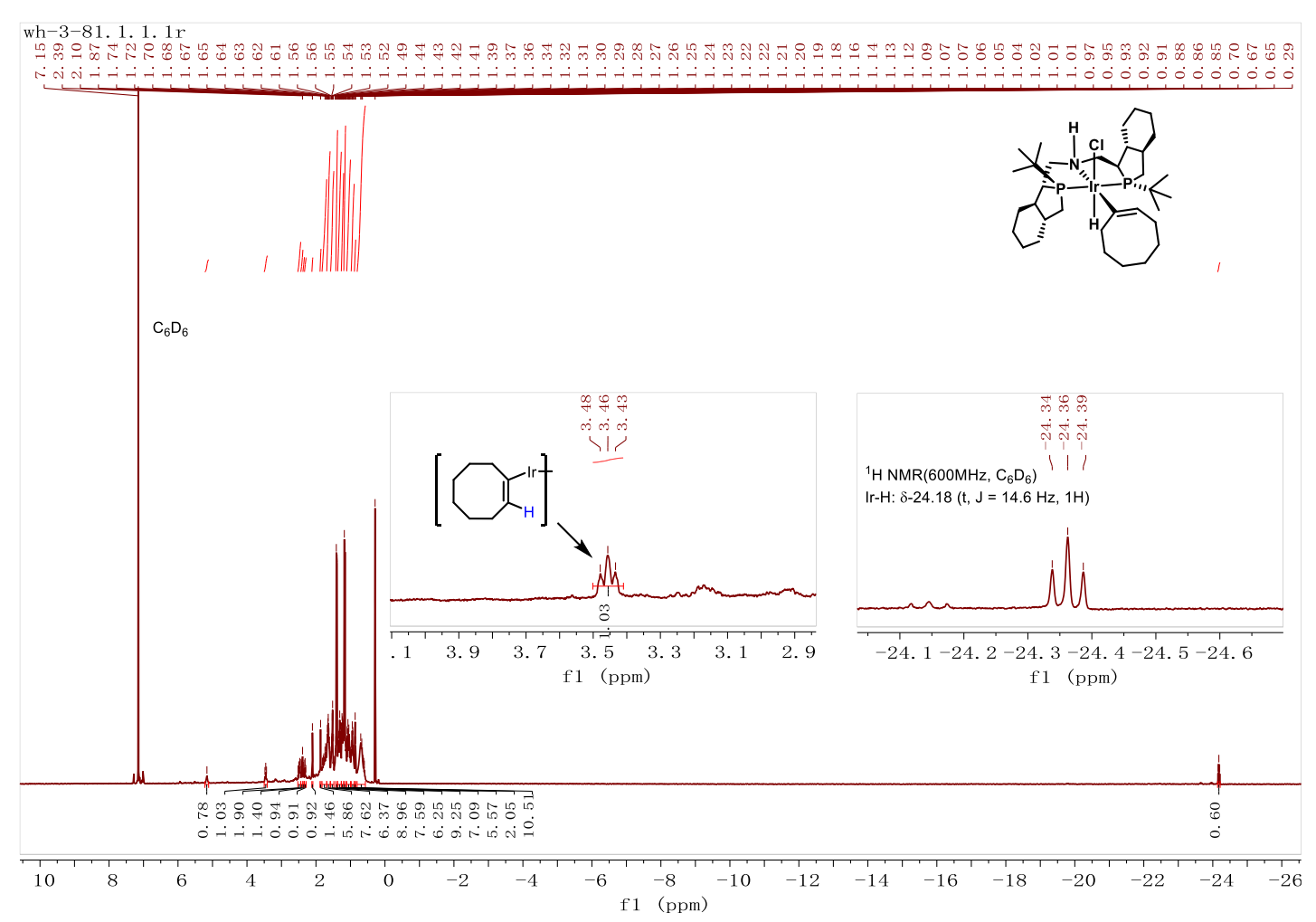

${ }^{31} \mathrm{P}$ NMR (243 MHz, $\left.\mathrm{C}_{6} \mathrm{D}_{6}\right)$ of crude Ir/Heng-PNP precatalyst
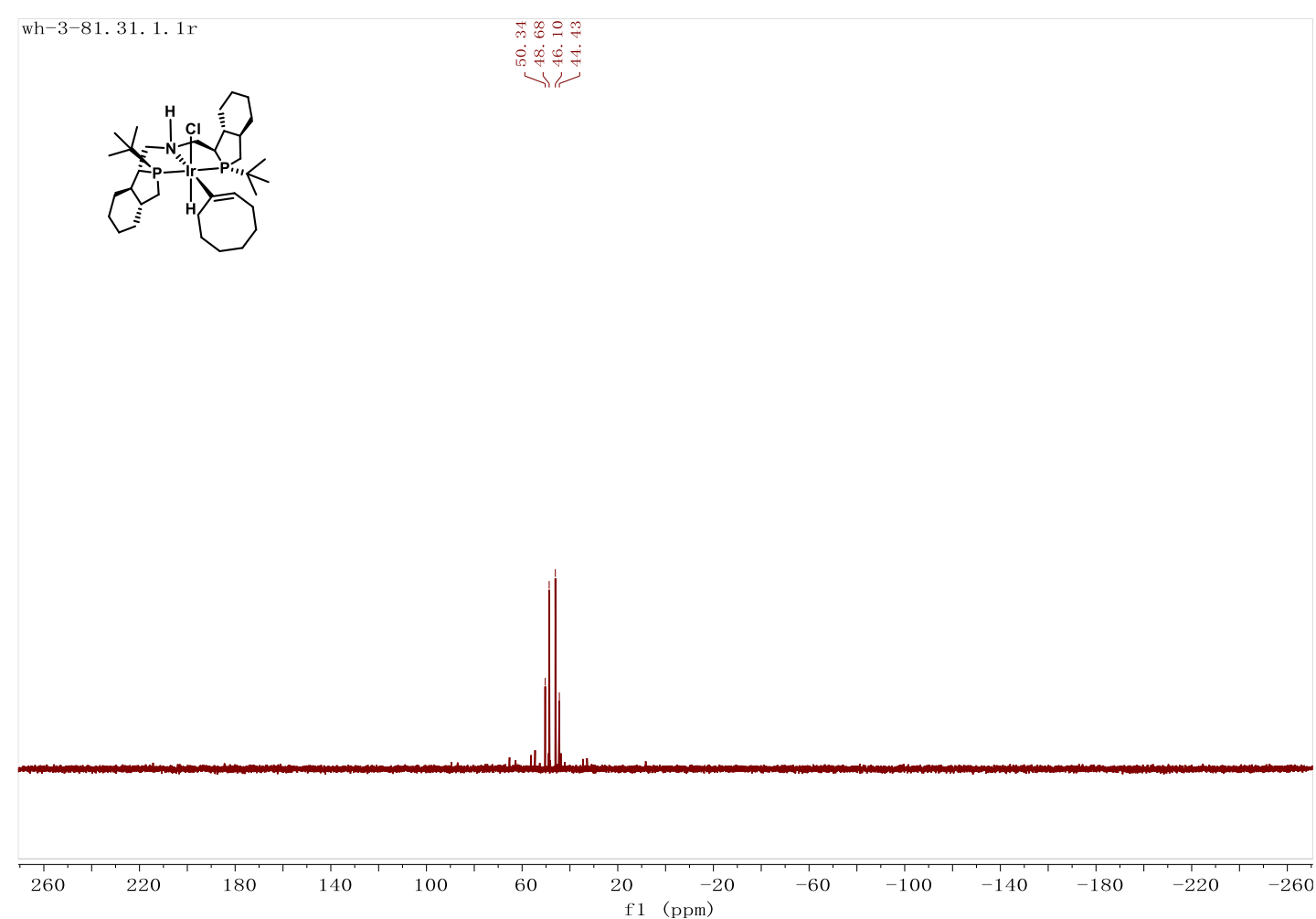
${ }^{1} \mathrm{H}$ NMR (600 MHz, $\left.\mathrm{CDCl}_{3}\right)$ of $9 \mathrm{c}$

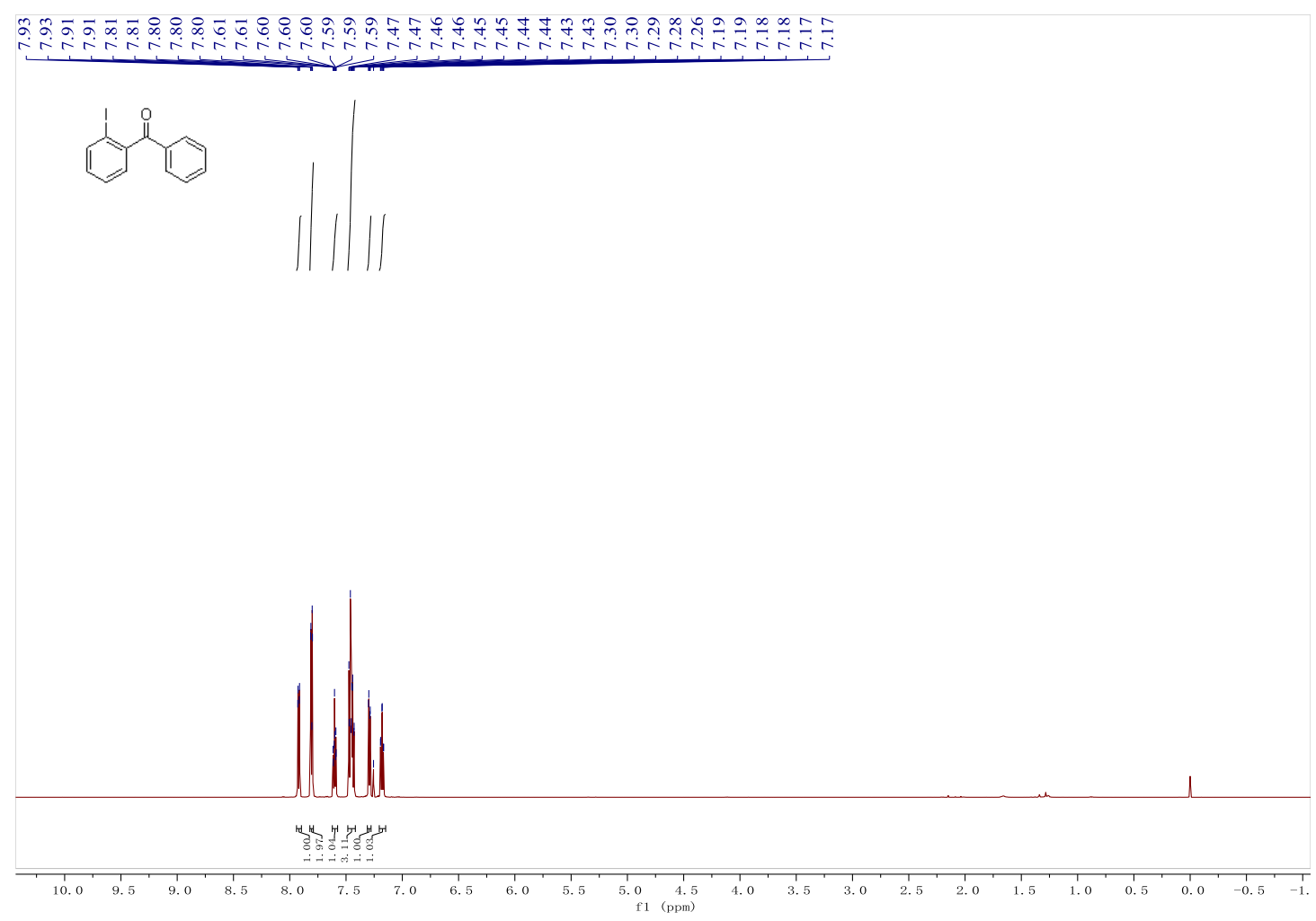

${ }^{13} \mathrm{C}$ NMR (151 MHz, $\left.\mathrm{CDCl}_{3}\right)$ of 9c
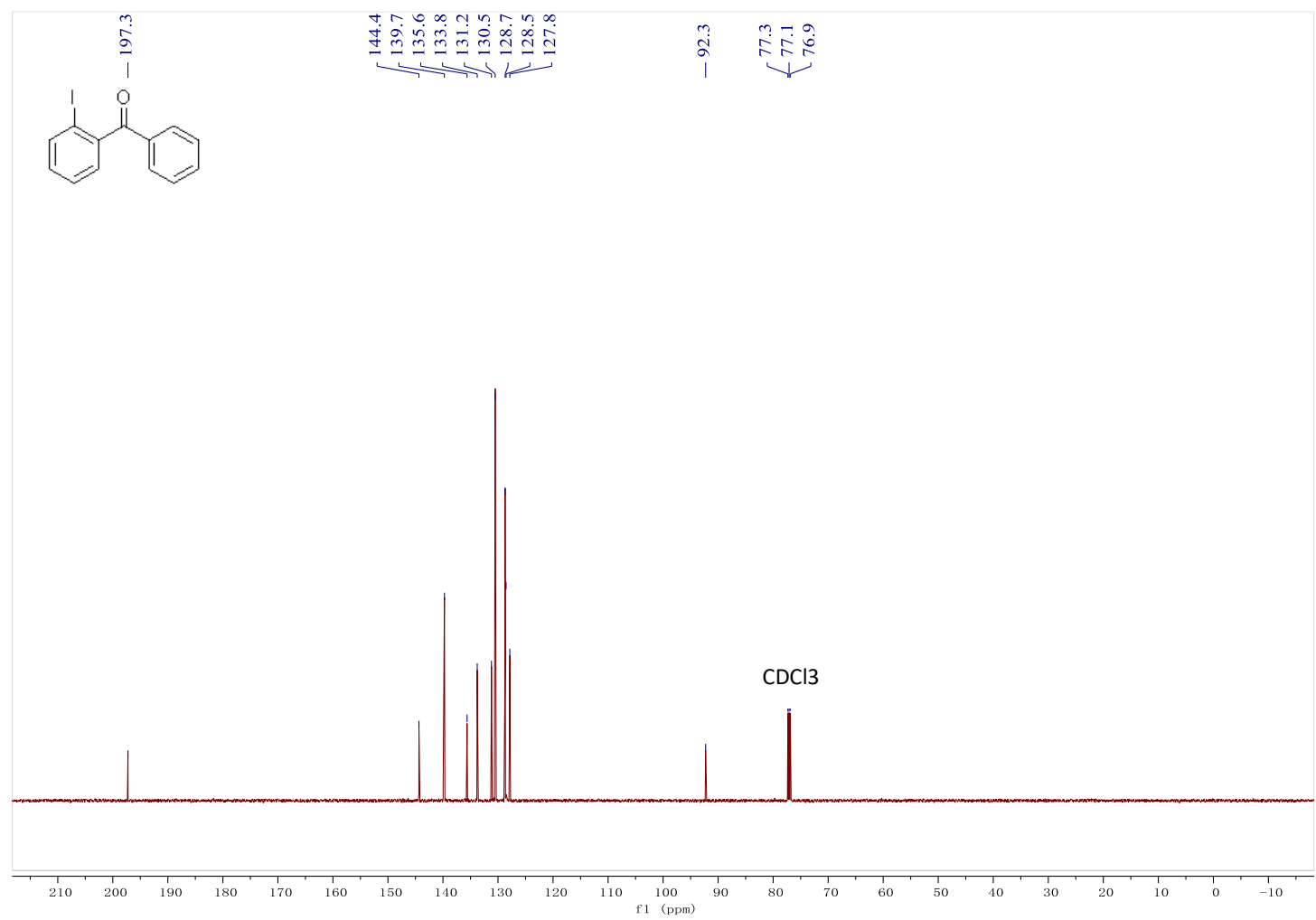
${ }^{1} \mathrm{H}$ NMR (400 MHz, $\left.\mathrm{CDCl}_{3}\right)$ of 9d
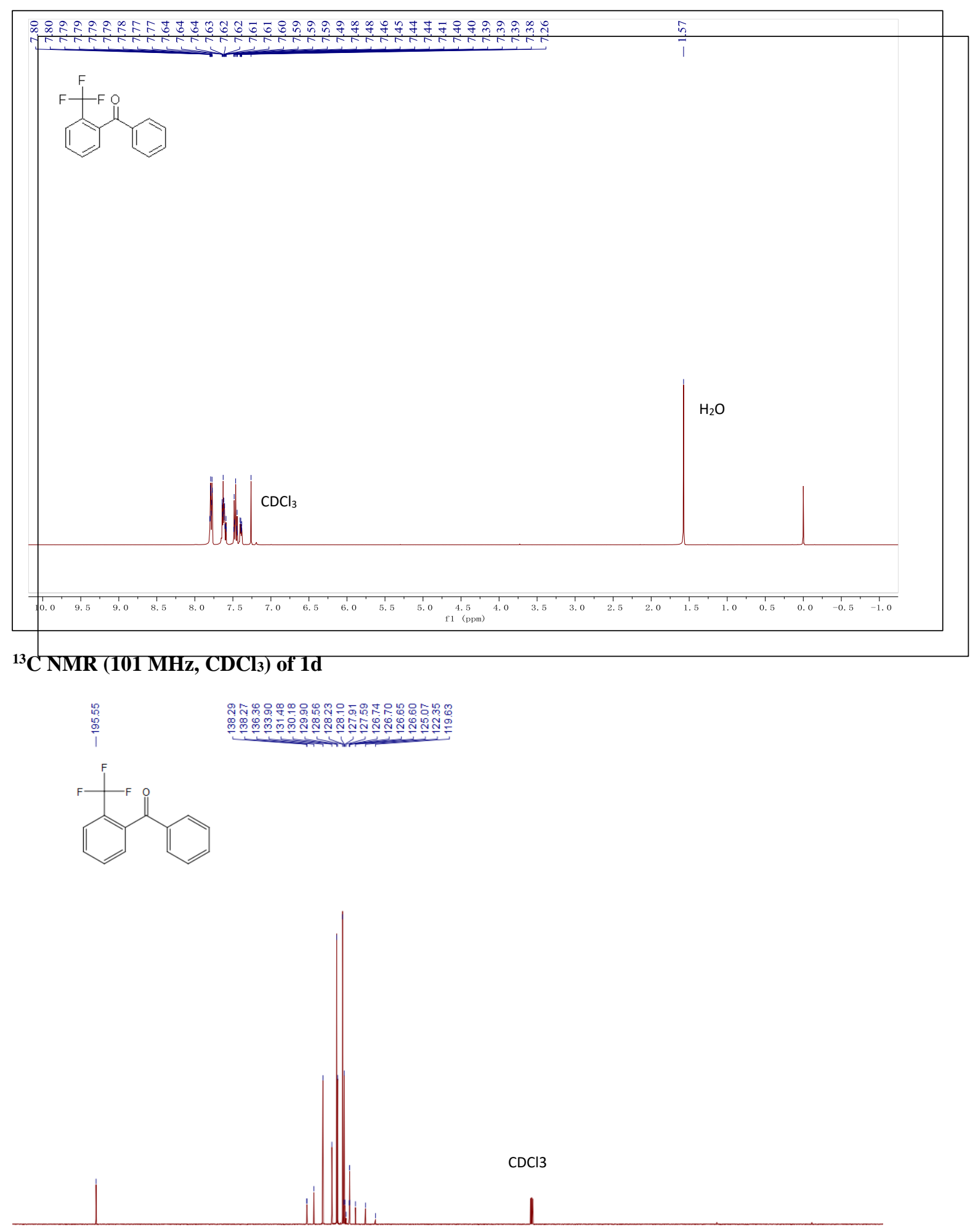

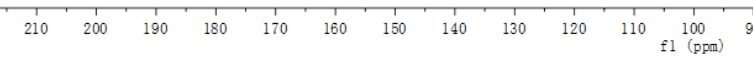


${ }^{1} \mathrm{H}$ NMR (400 MHz, $\left.\mathrm{CDCl}_{3}\right)$ of $9 \mathrm{f}$

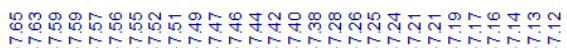
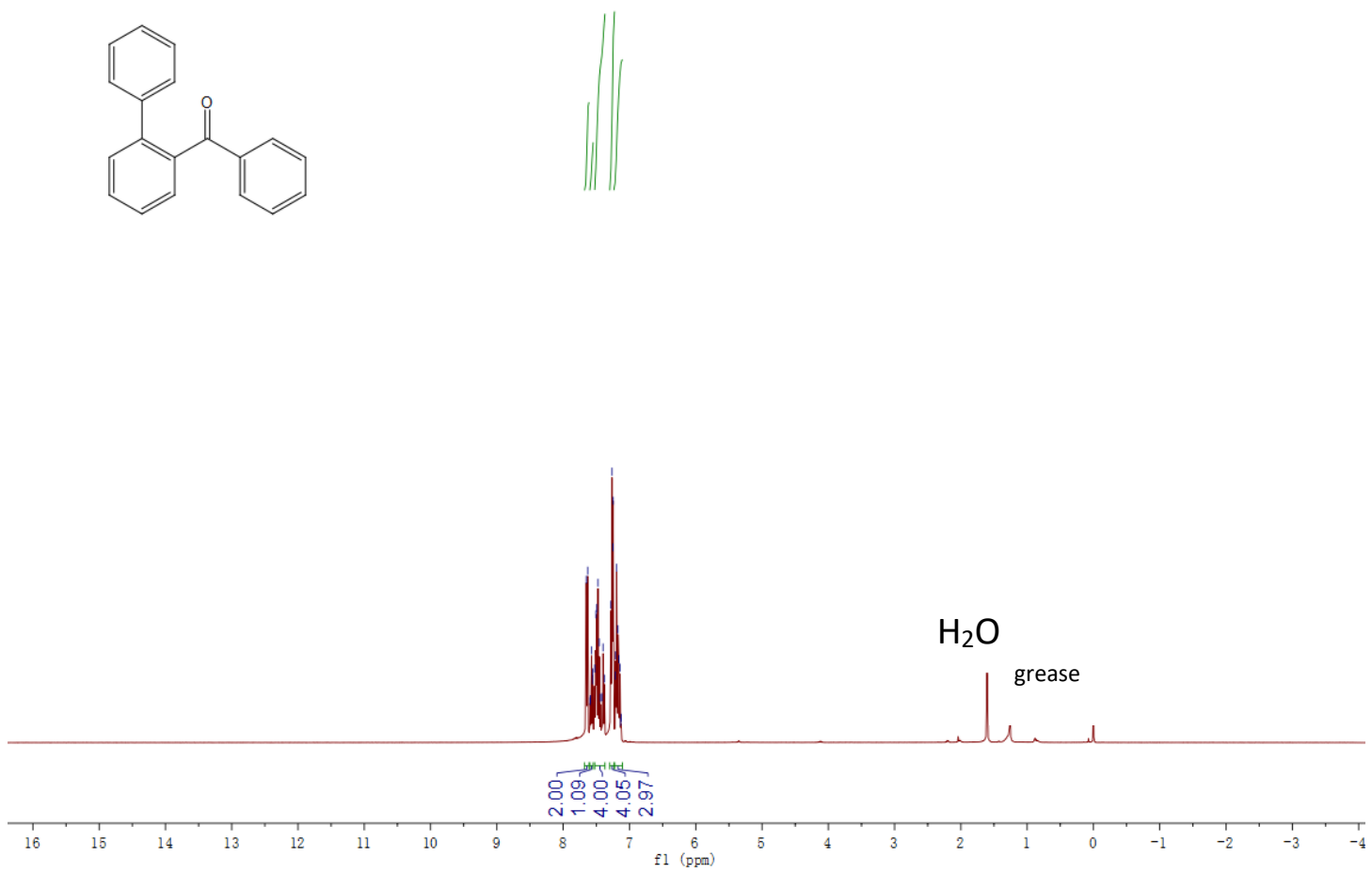

${ }^{13} \mathrm{C}$ NMR (101 MHz, $\left.\mathrm{CDCl}_{3}\right)$ of $9 \mathrm{f}$
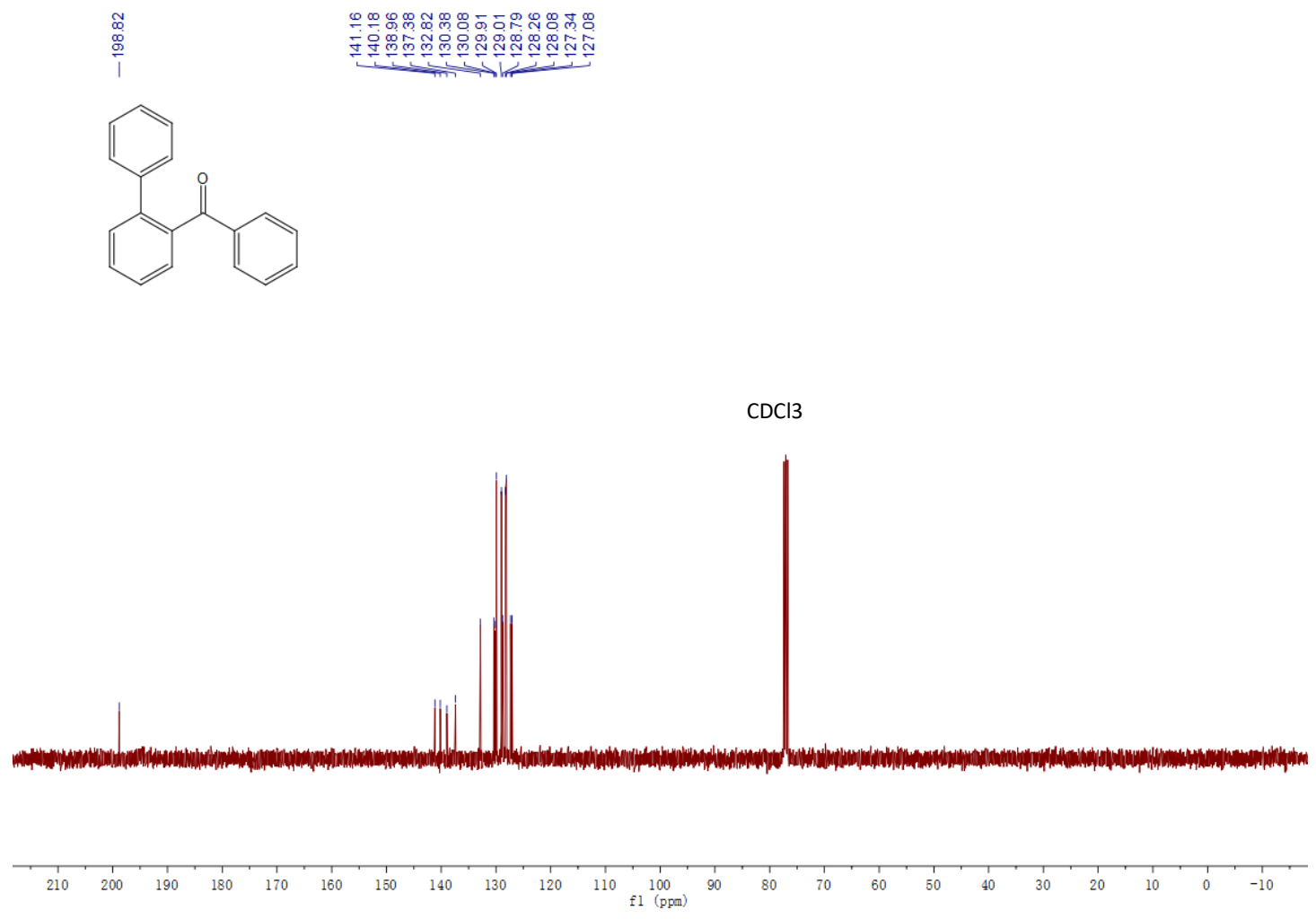

S61 
${ }^{1} \mathrm{H}$ NMR (400 MHz, CDCl $)$ of $9 \mathrm{~g}$

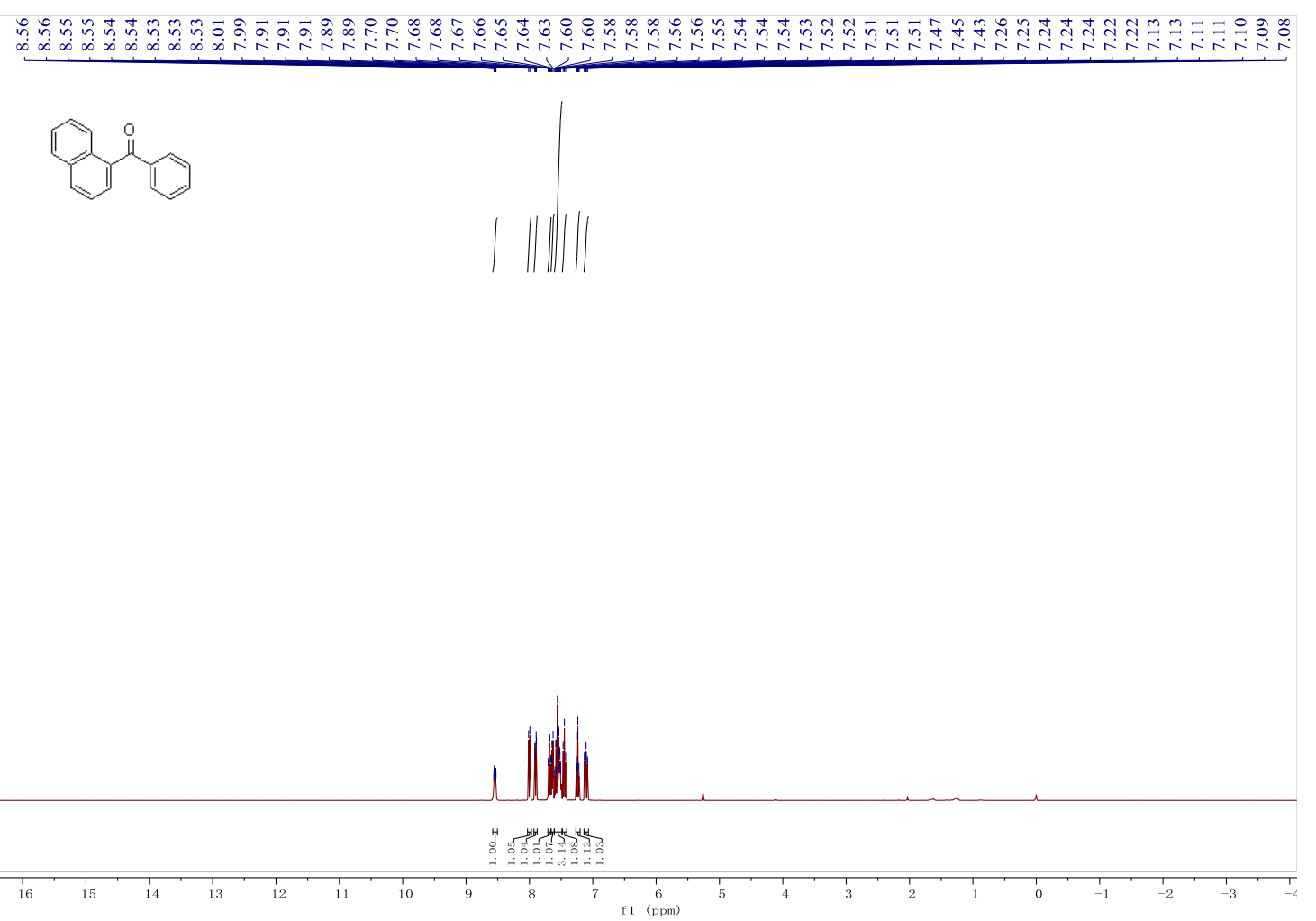

${ }^{13} \mathrm{C}$ NMR (151 MHz, CDCl 3$)$ of 9g

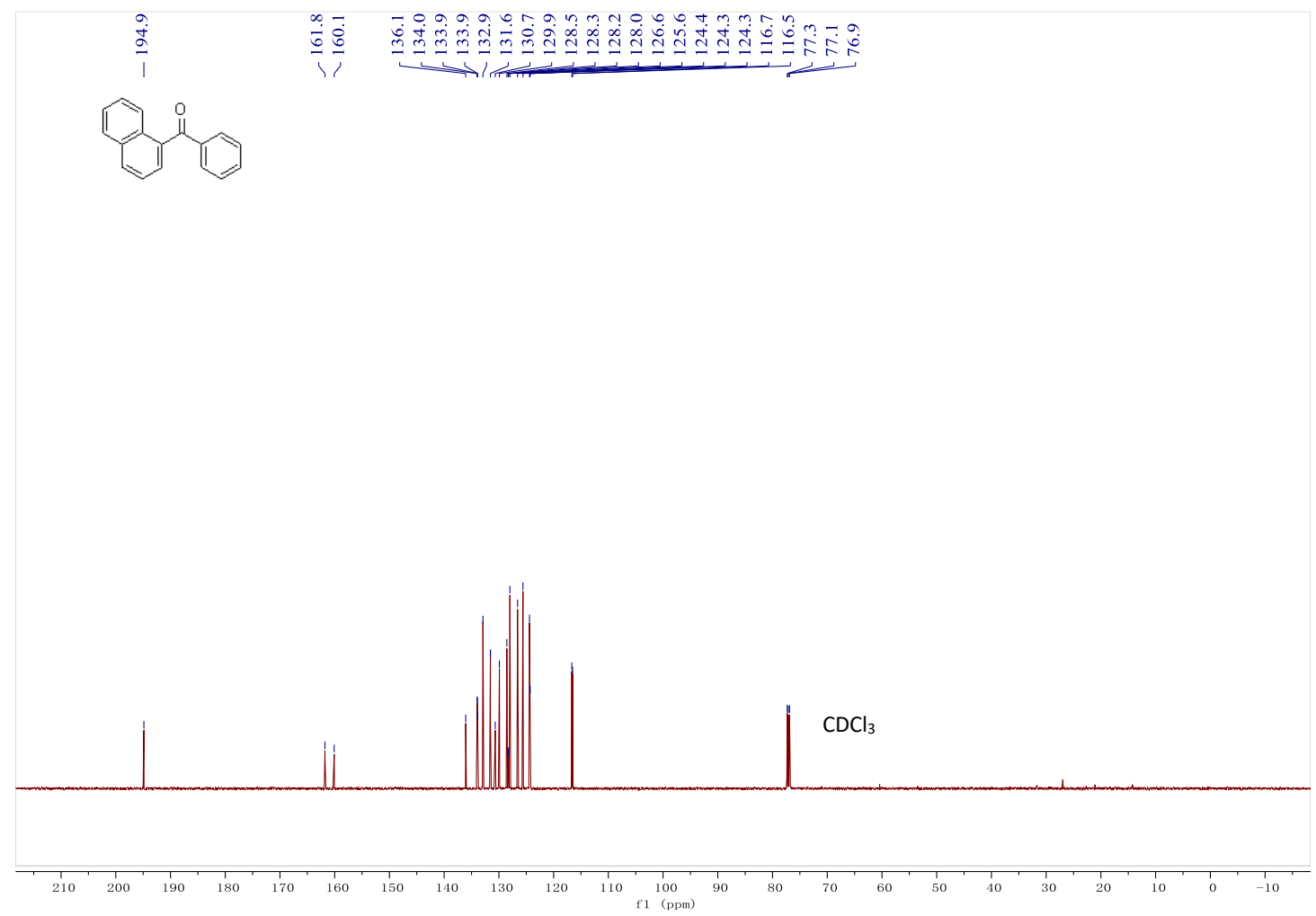


${ }^{1} \mathrm{H}$ NMR (600 MHz, CDCl 3$)$ of $9 \mathrm{~h}$

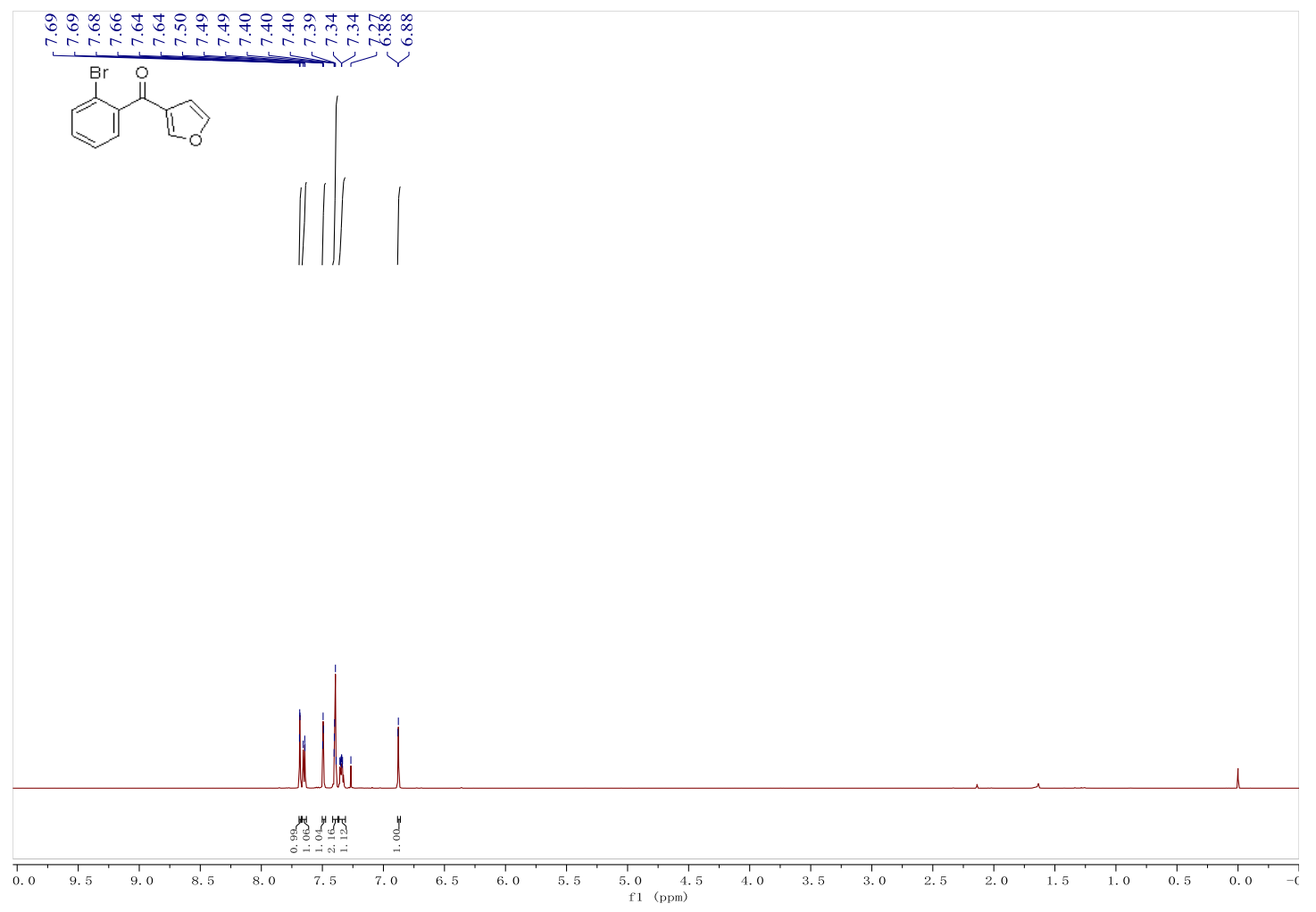

${ }^{13} \mathrm{C}$ NMR (151 MHz, CDCl 3$)$ of $9 \mathrm{~h}$
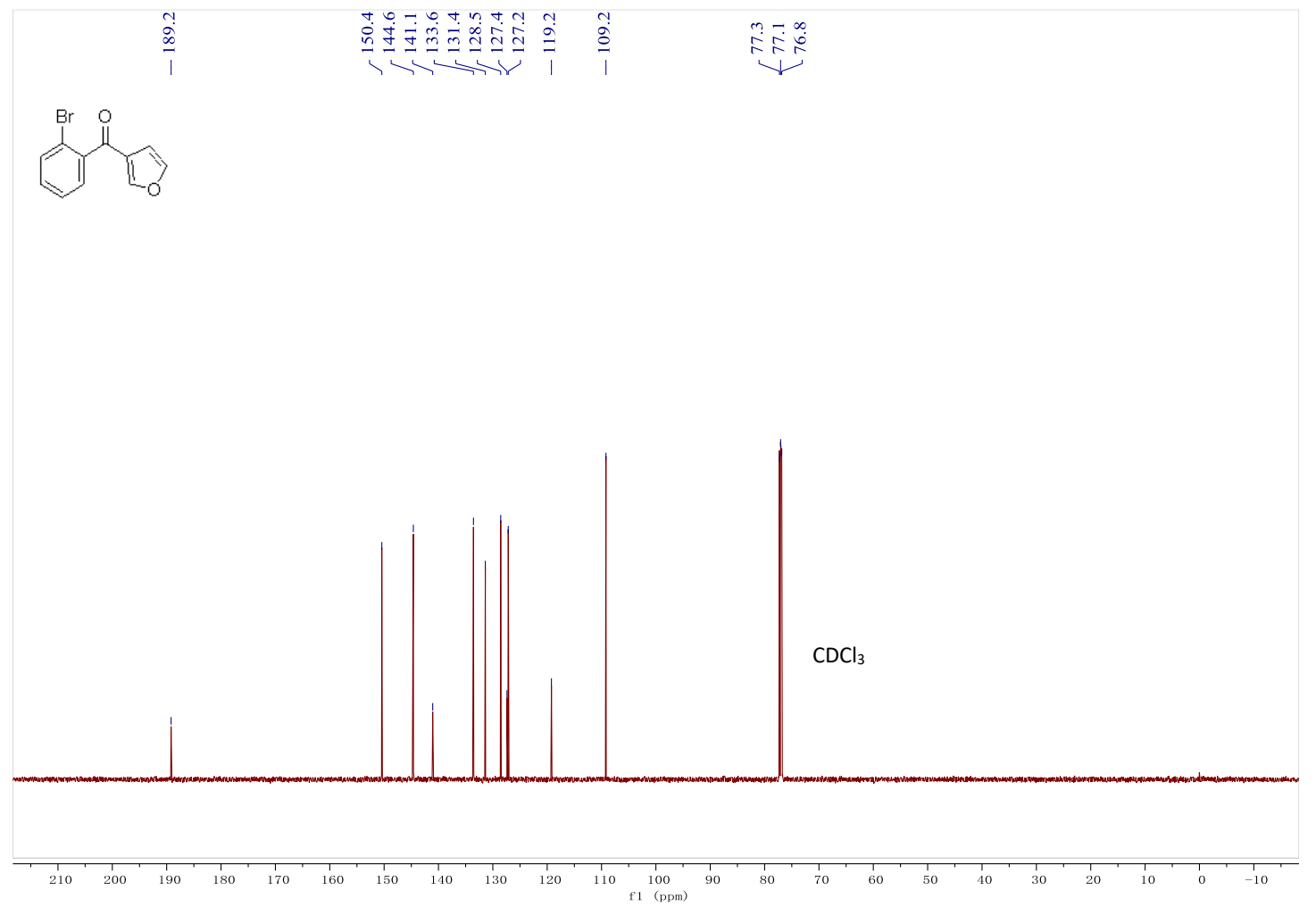
${ }^{1} \mathrm{H}$ NMR (400 MHz, $\left.\mathrm{CDCl}_{3}\right)$ of $9 \mathrm{i}$

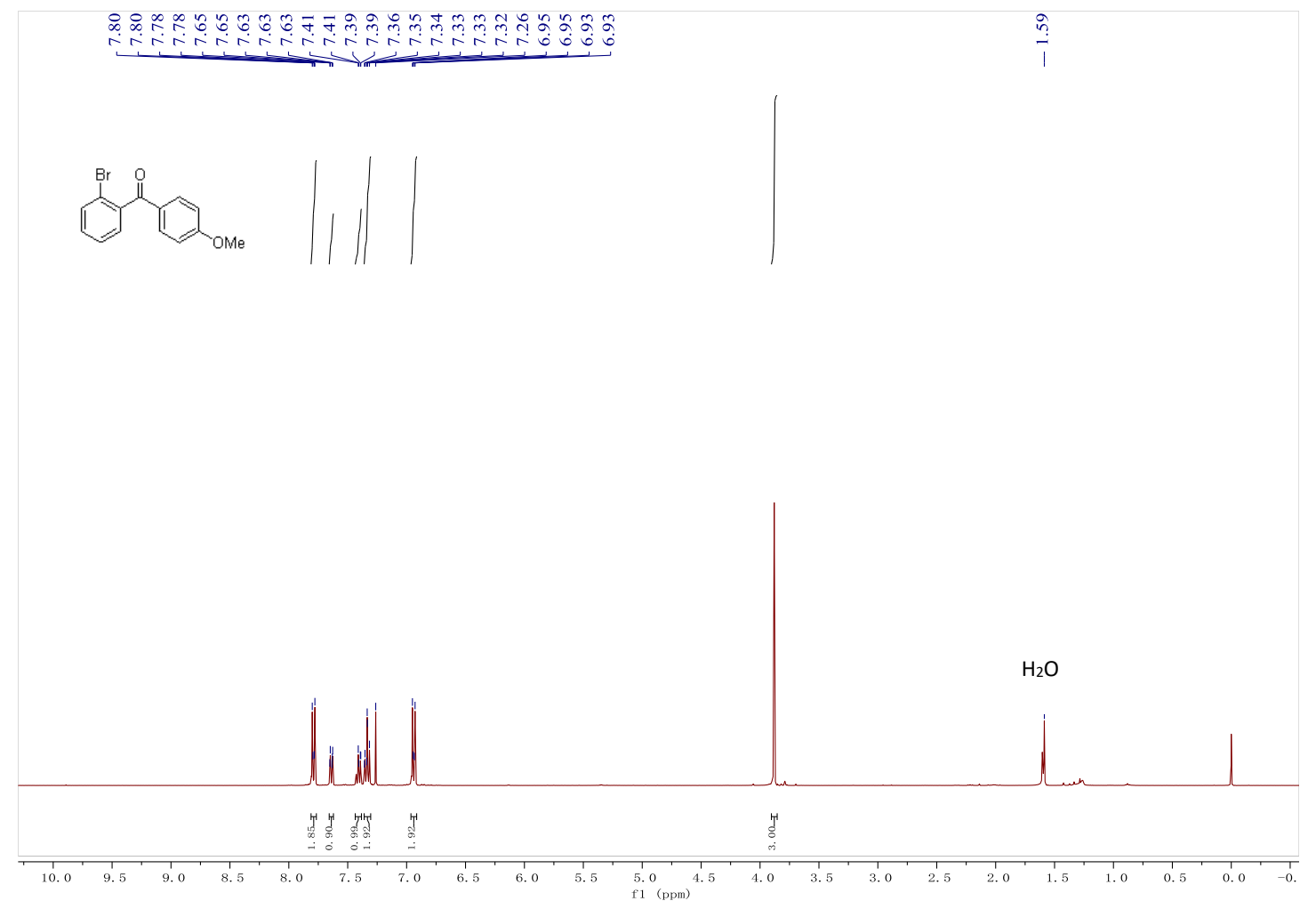

${ }^{13} \mathrm{C}$ NMR (101 MHz, CDCl 3$)$ of 9i
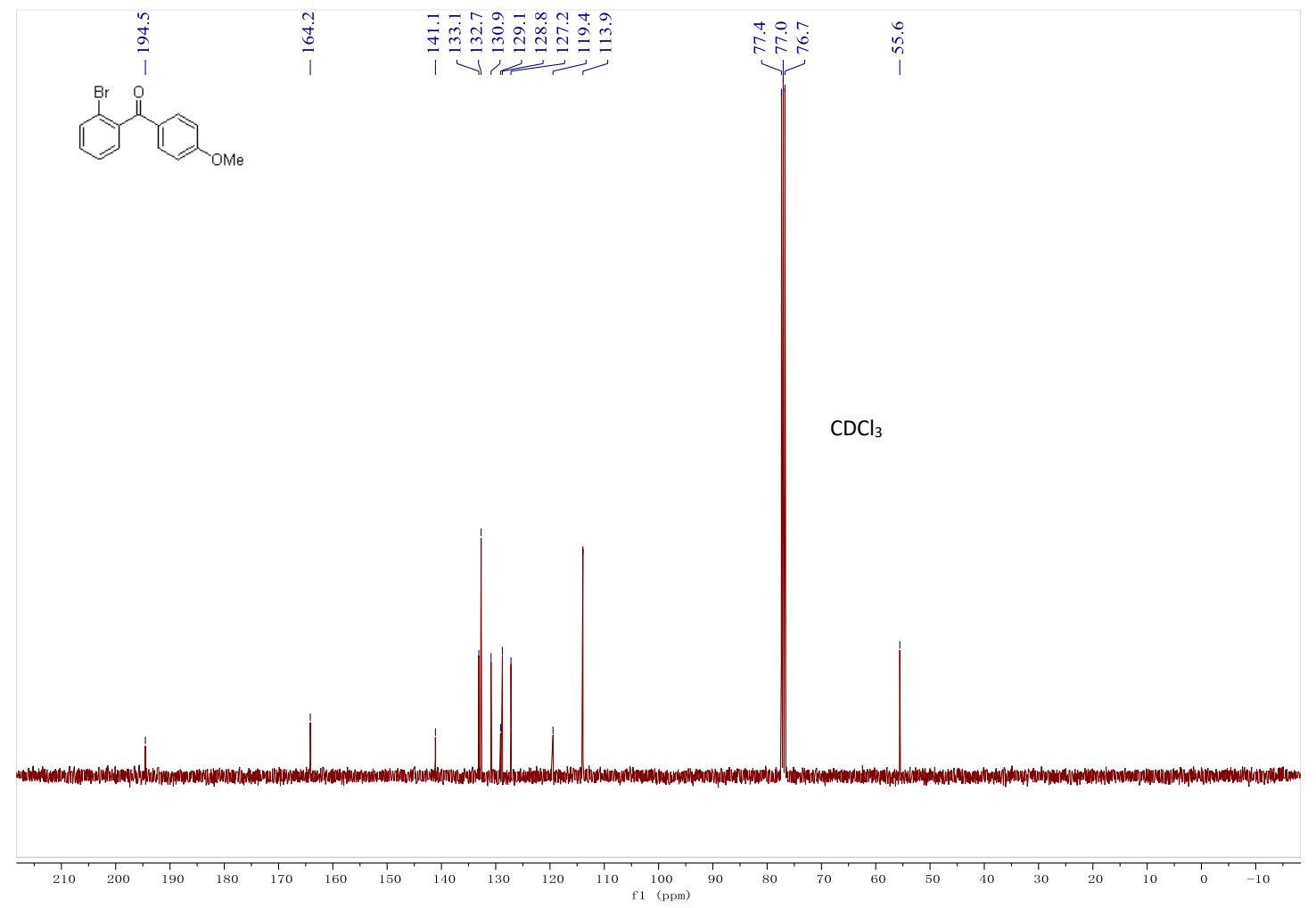
${ }^{1} \mathrm{H}$ NMR (600 MHz, $\left.\mathrm{CDCl}_{3}\right)$ of $9 \mathrm{j}$

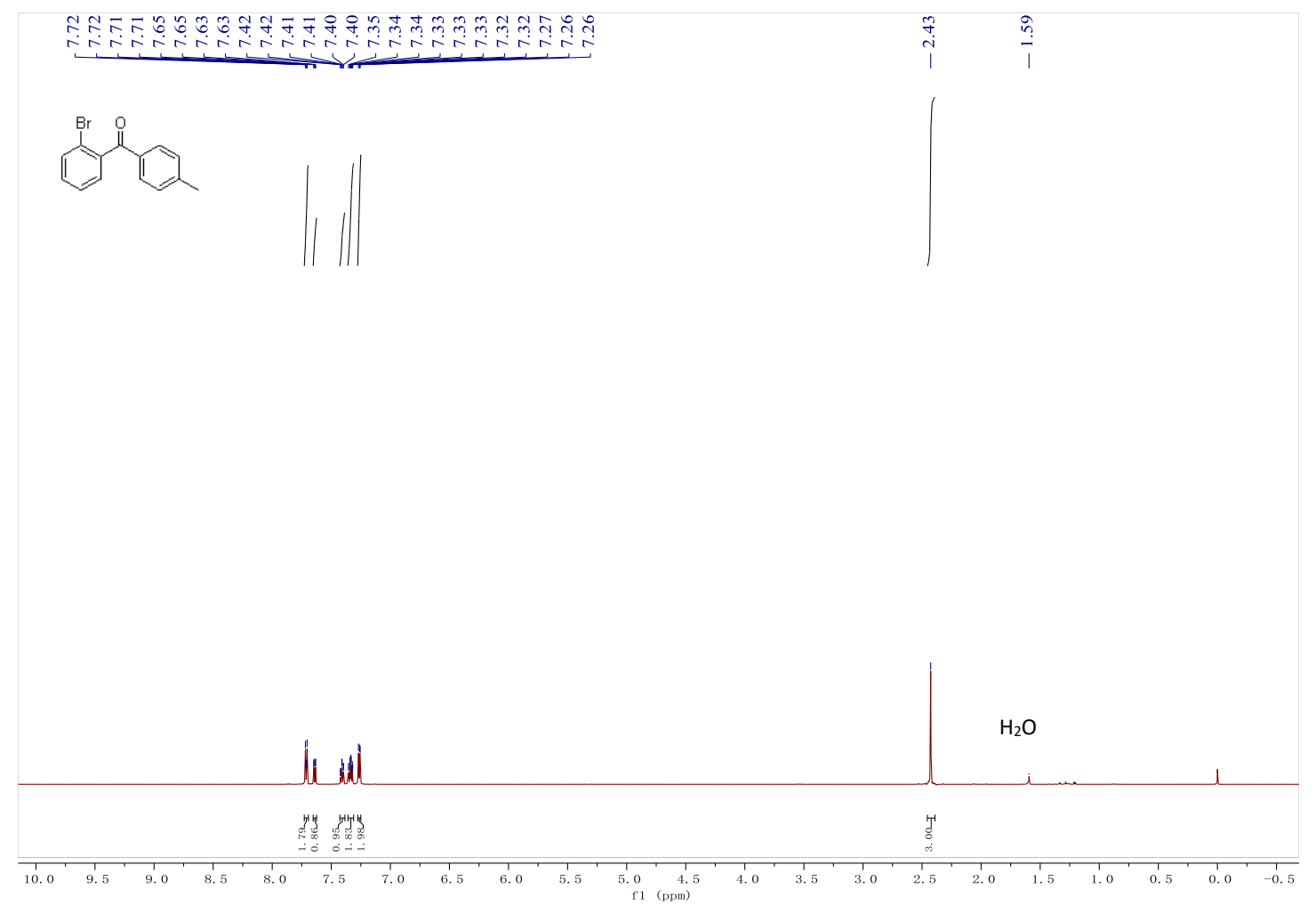

${ }^{13} \mathrm{C}$ NMR (151 MHz, $\left.\mathrm{CDCl}_{3}\right)$ of 9j

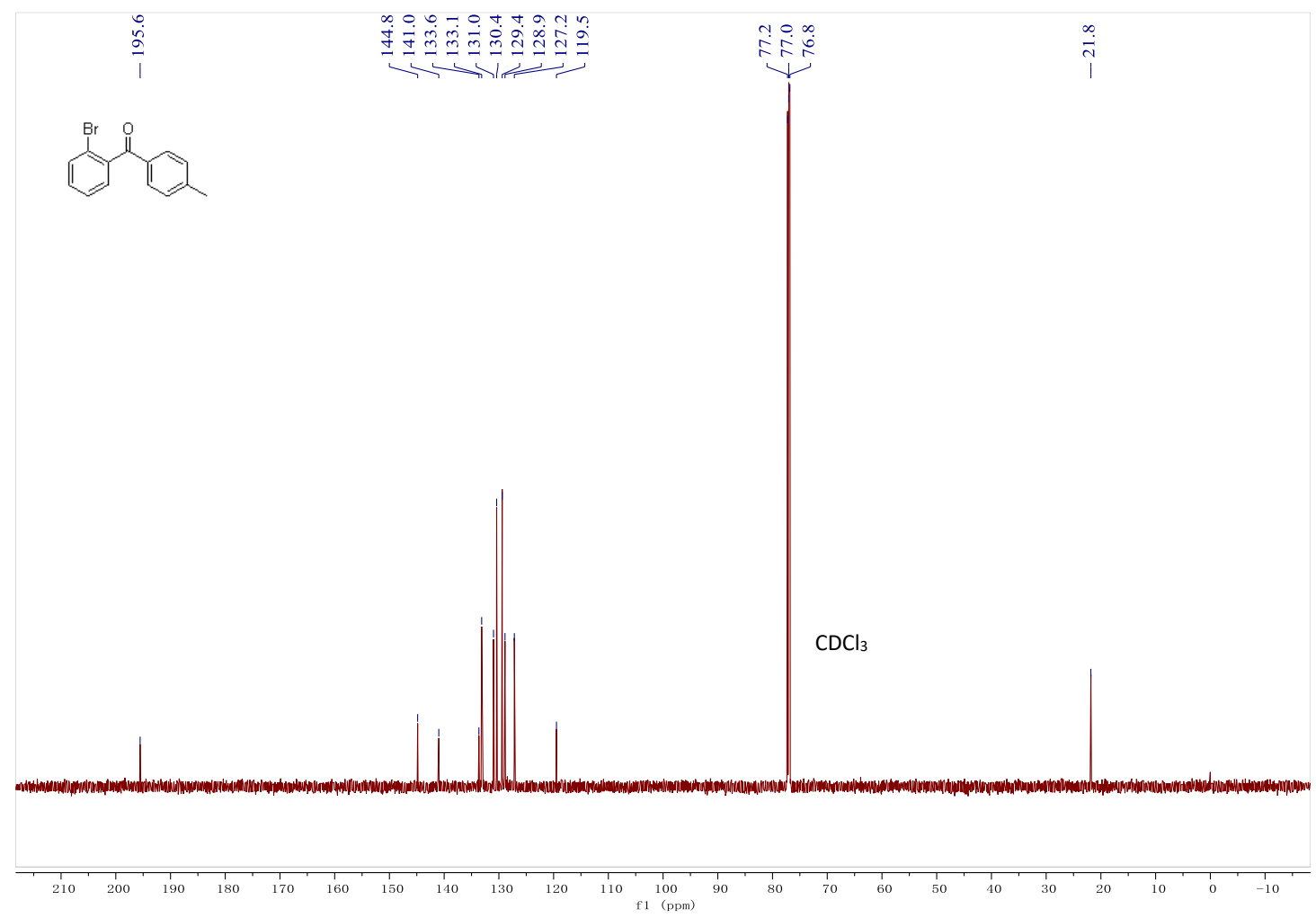


${ }^{1} \mathrm{H}$ NMR (400 MHz, $\left.\mathrm{CDCl}_{3}\right)$ of $9 \mathrm{k}$

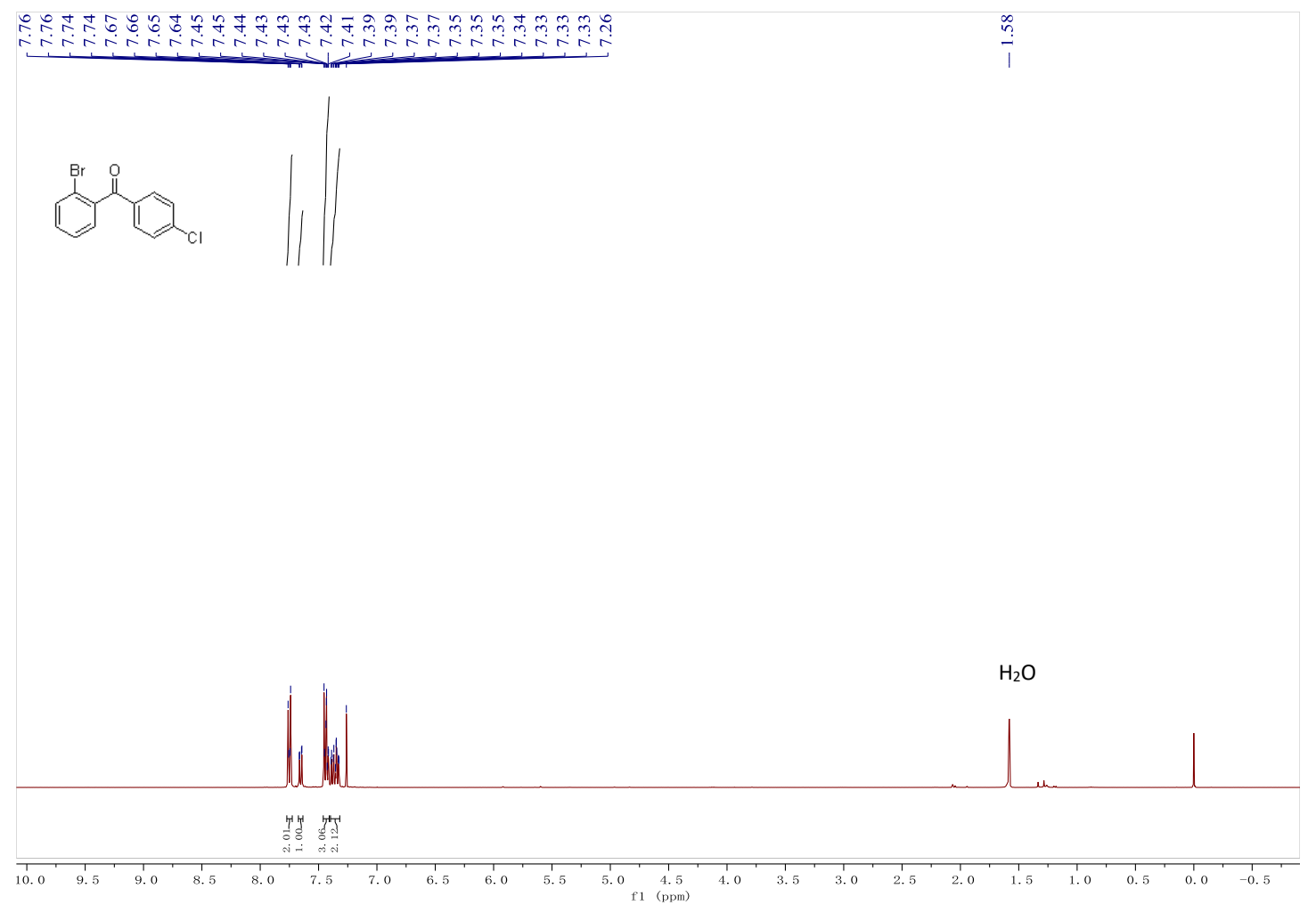

${ }^{13} \mathrm{C}$ NMR (101 MHz, $\left.\mathrm{CDCl}_{3}\right)$ of 9k
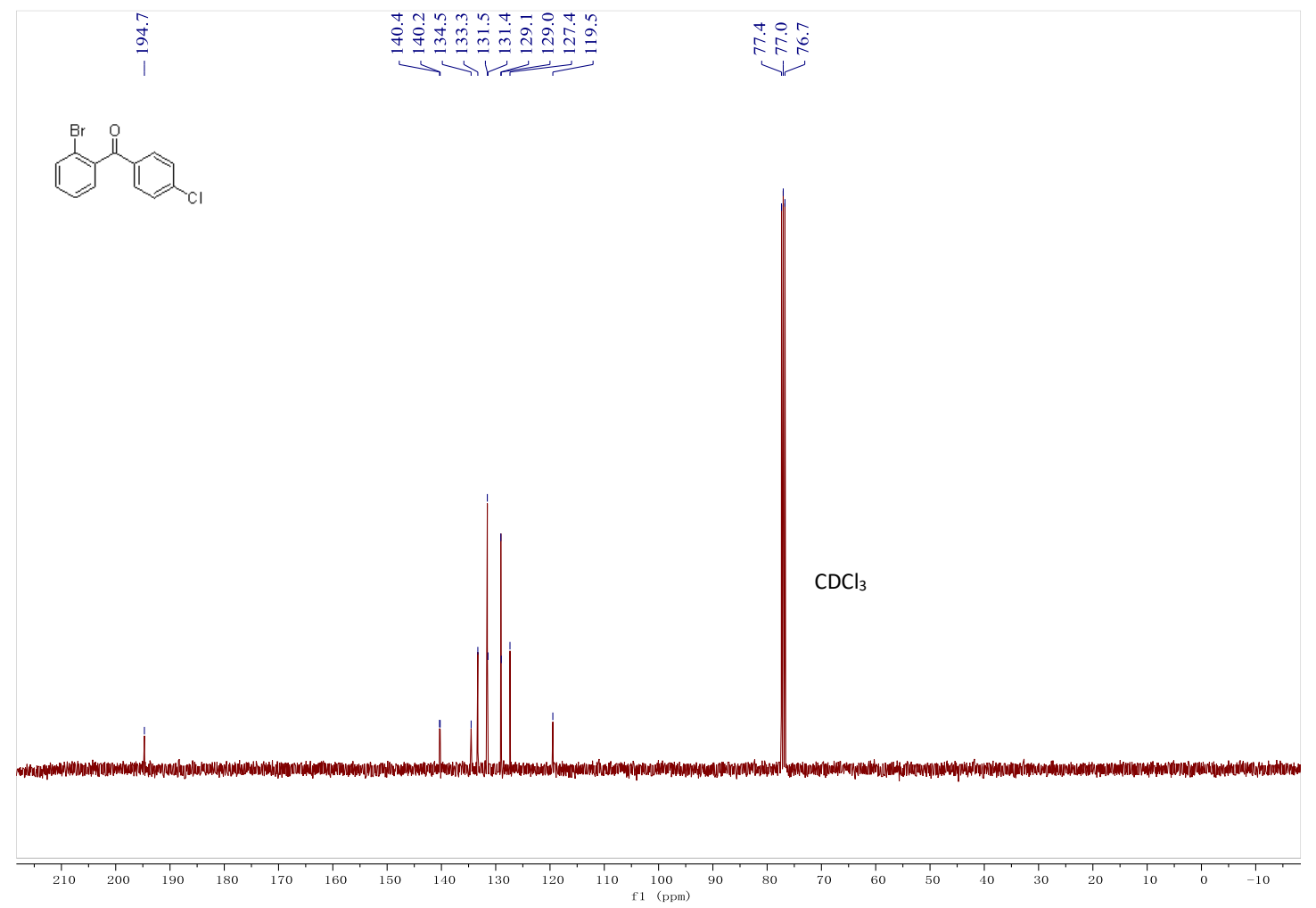
${ }^{1} \mathrm{H}$ NMR (400 MHz, $\left.\mathrm{CDCl}_{3}\right)$ of 91

$\underbrace{0}$
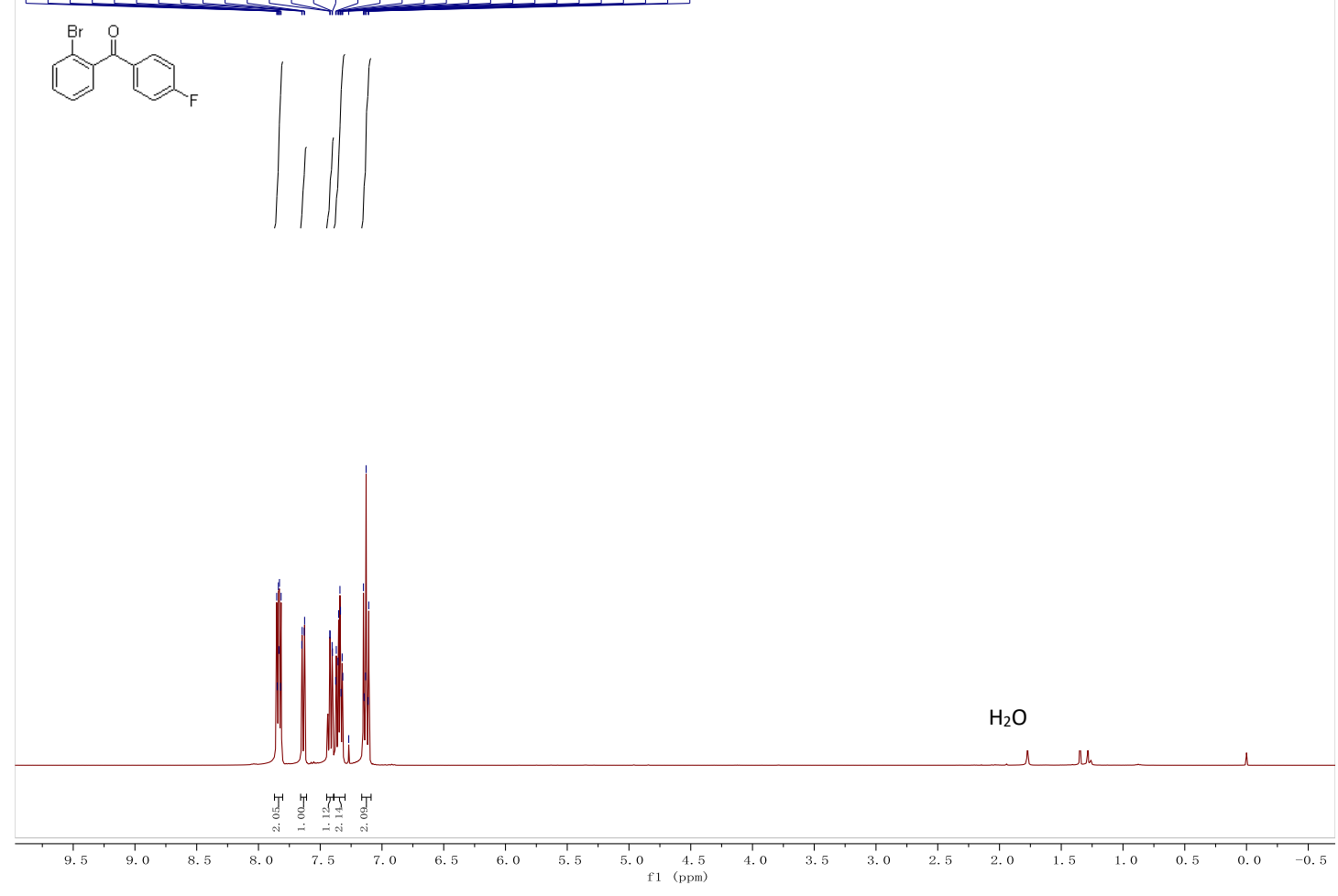

${ }^{13} \mathrm{C}$ NMR (101 MHz, $\left.\mathrm{CDCl}_{3}\right)$ of 91
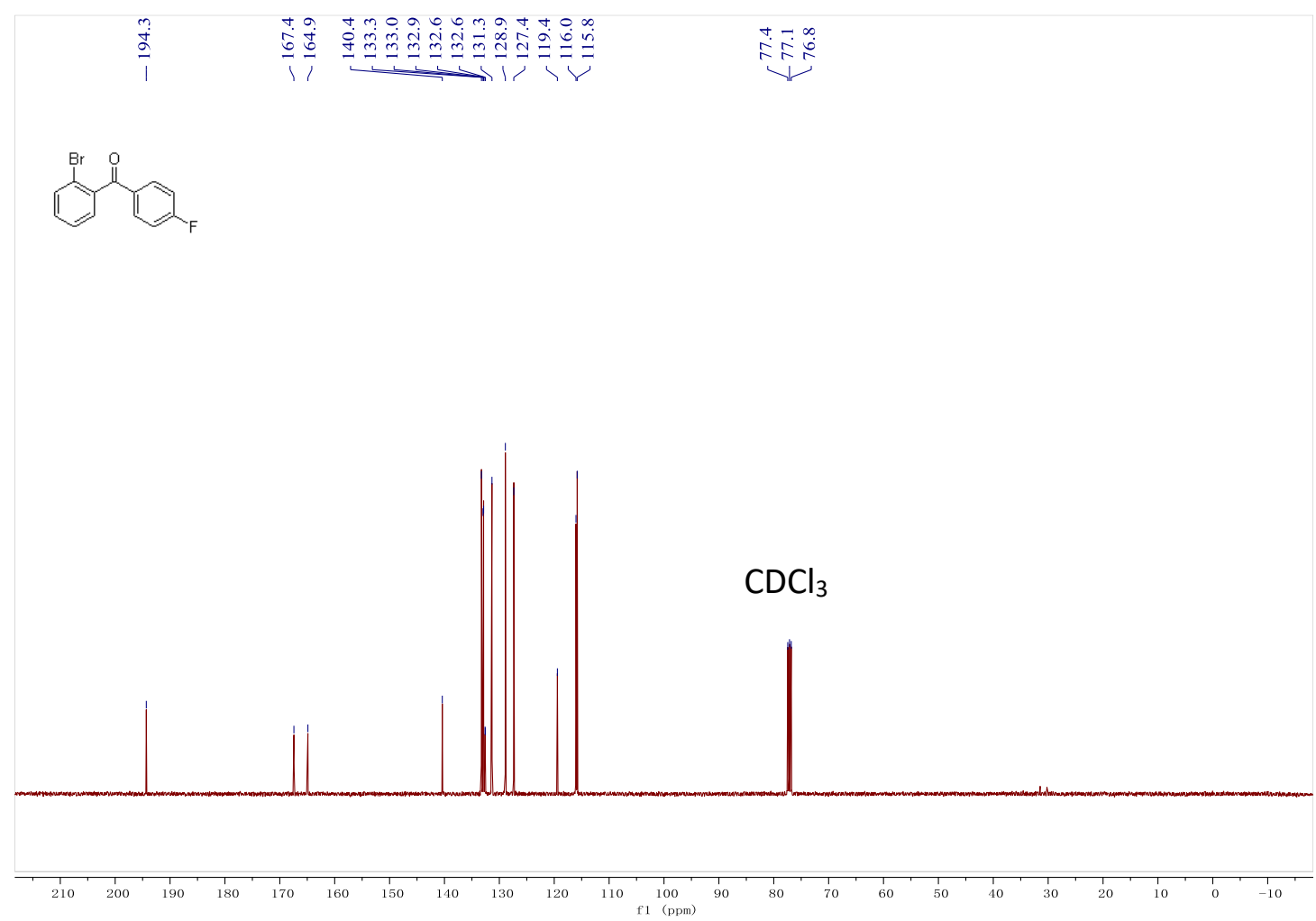
${ }^{1} \mathrm{H}$ NMR (600 MHz, CDCl 3$)$ of $9 \mathrm{~m}$

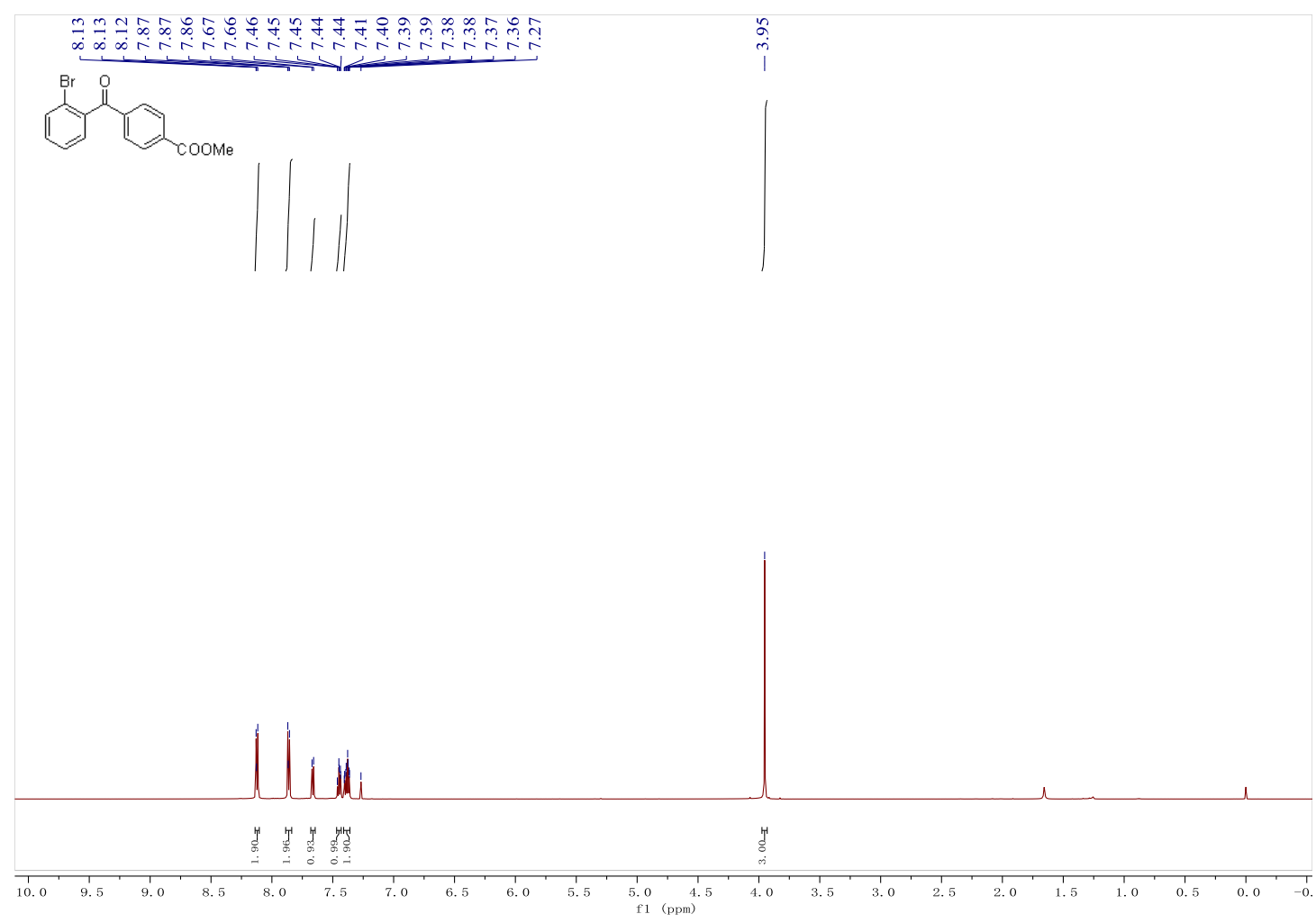

${ }^{13} \mathrm{C}$ NMR (151 MHz, CDCl $)$ of 9m
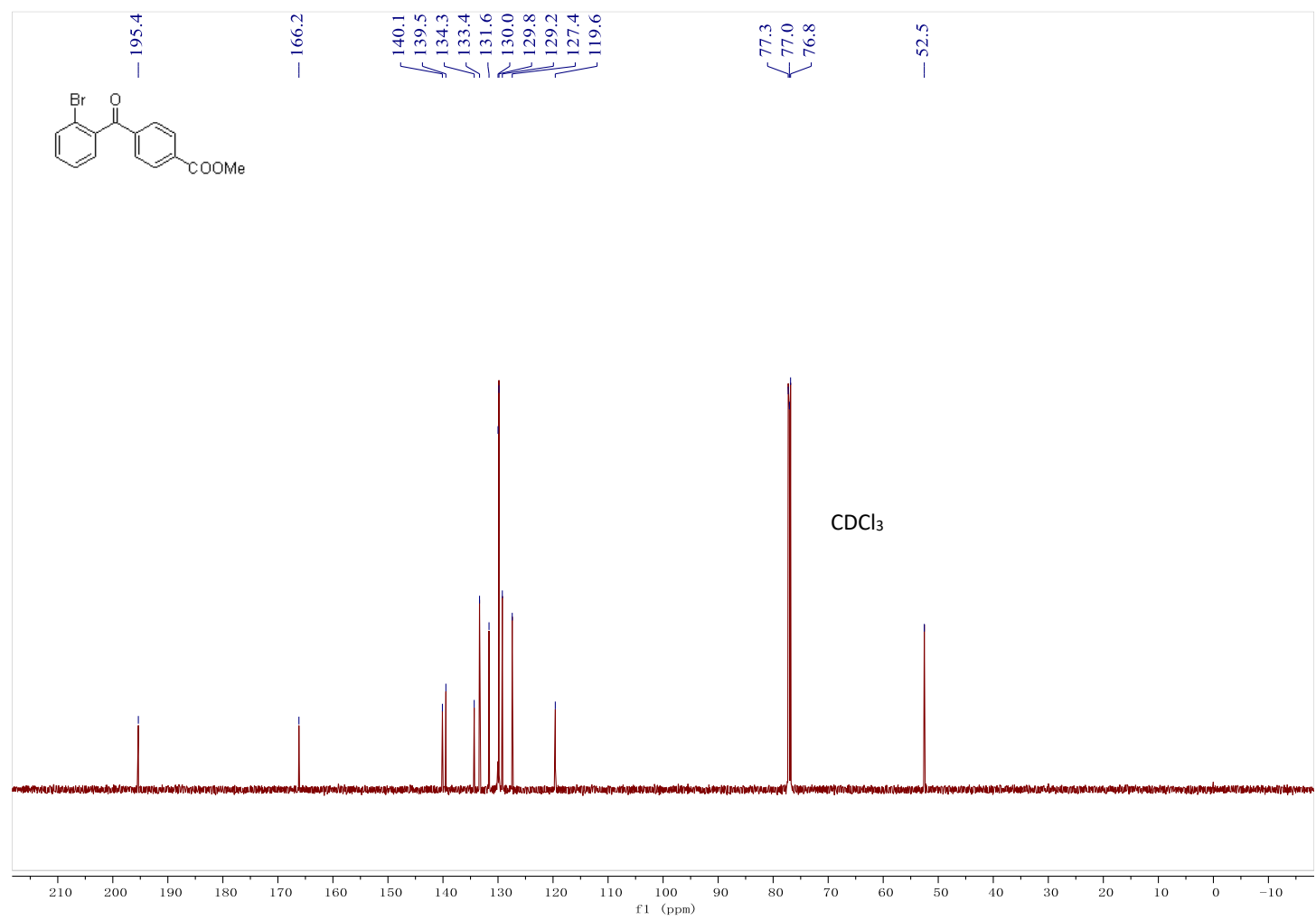
${ }^{1} \mathrm{H}$ NMR (600 MHz, CDCl 3$)$ of 9n

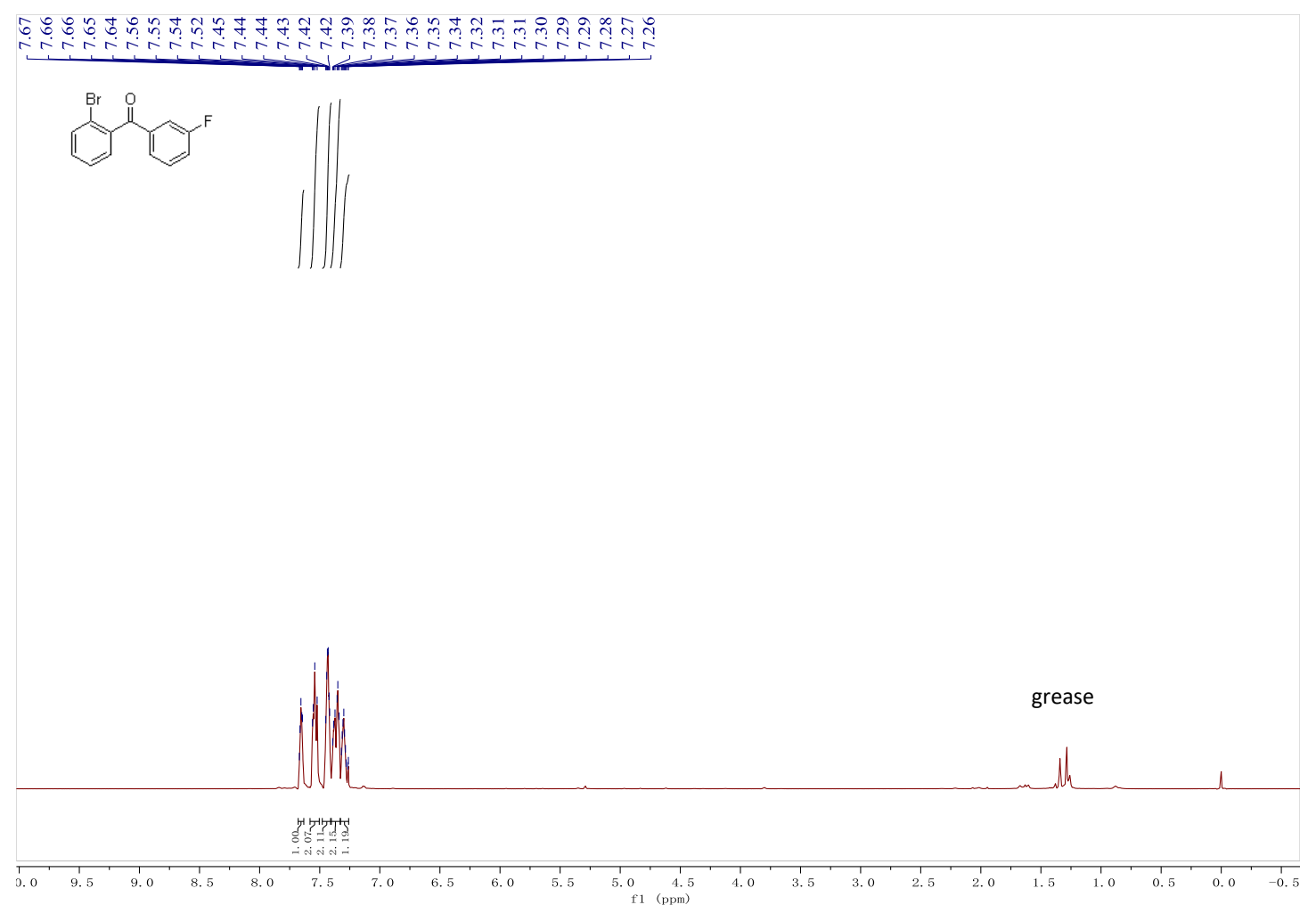

${ }^{13} \mathrm{C}$ NMR (151 MHz, CDCl 3$)$ of 9n

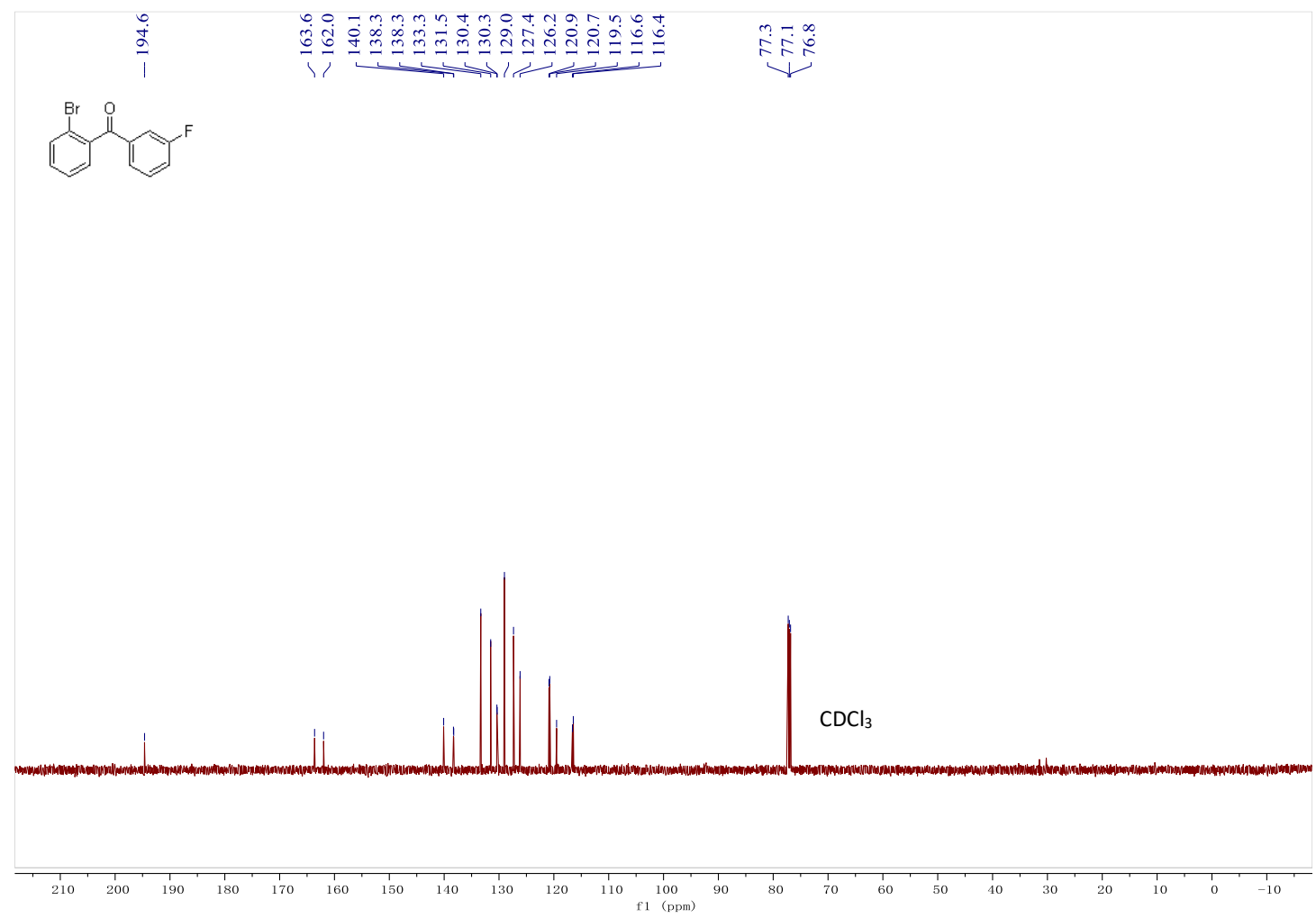


${ }^{1} \mathrm{H}$ NMR (400 MHz, $\mathrm{CDCl}_{3}$ ) of 90

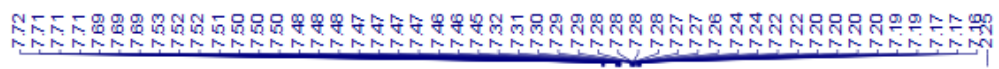
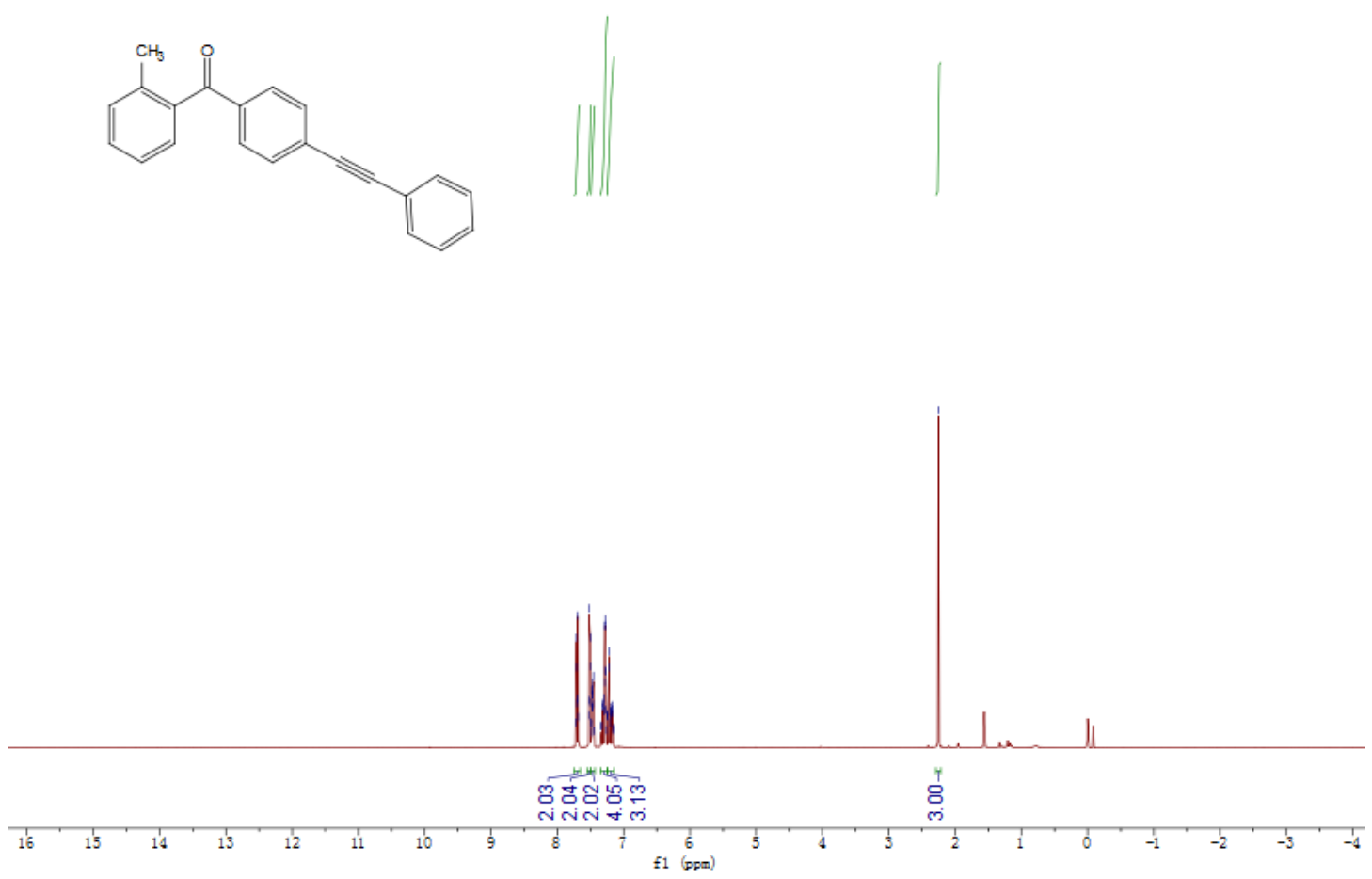

${ }^{13} \mathrm{C}$ NMR (101 MHz, $\left.\mathrm{CDCl}_{3}\right)$ of 90

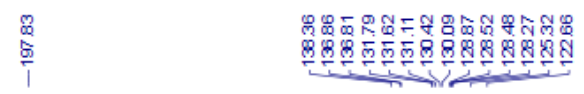

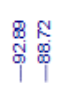

$\underset{\substack{8 \\ \text { i }}}{2}$
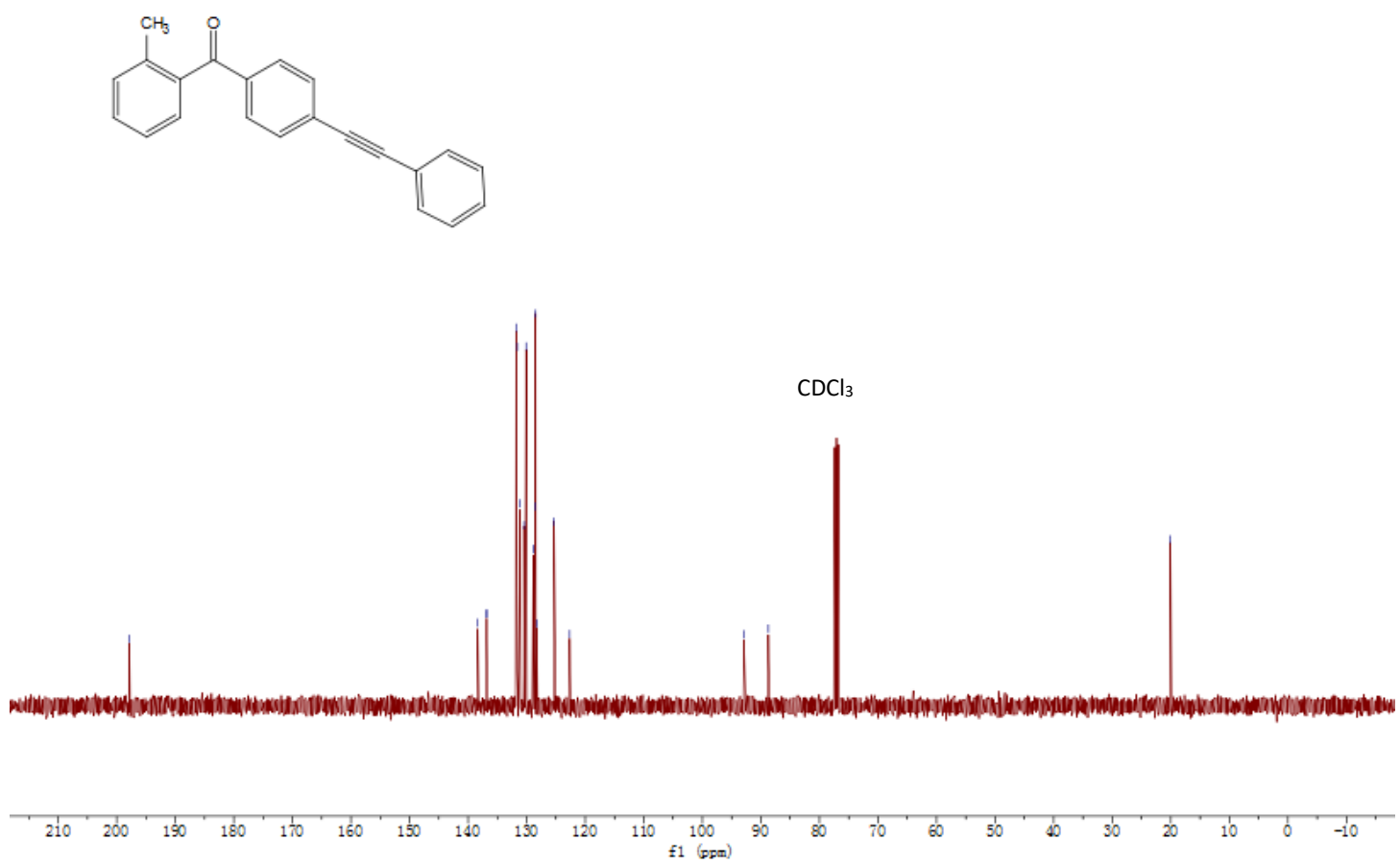

S70 
${ }^{1} \mathrm{H}$ NMR (400 MHz, $\mathrm{CDCl}_{3}$ ) of Pp

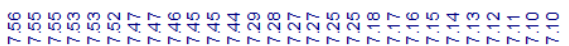

$\overline{\tilde{N}}$
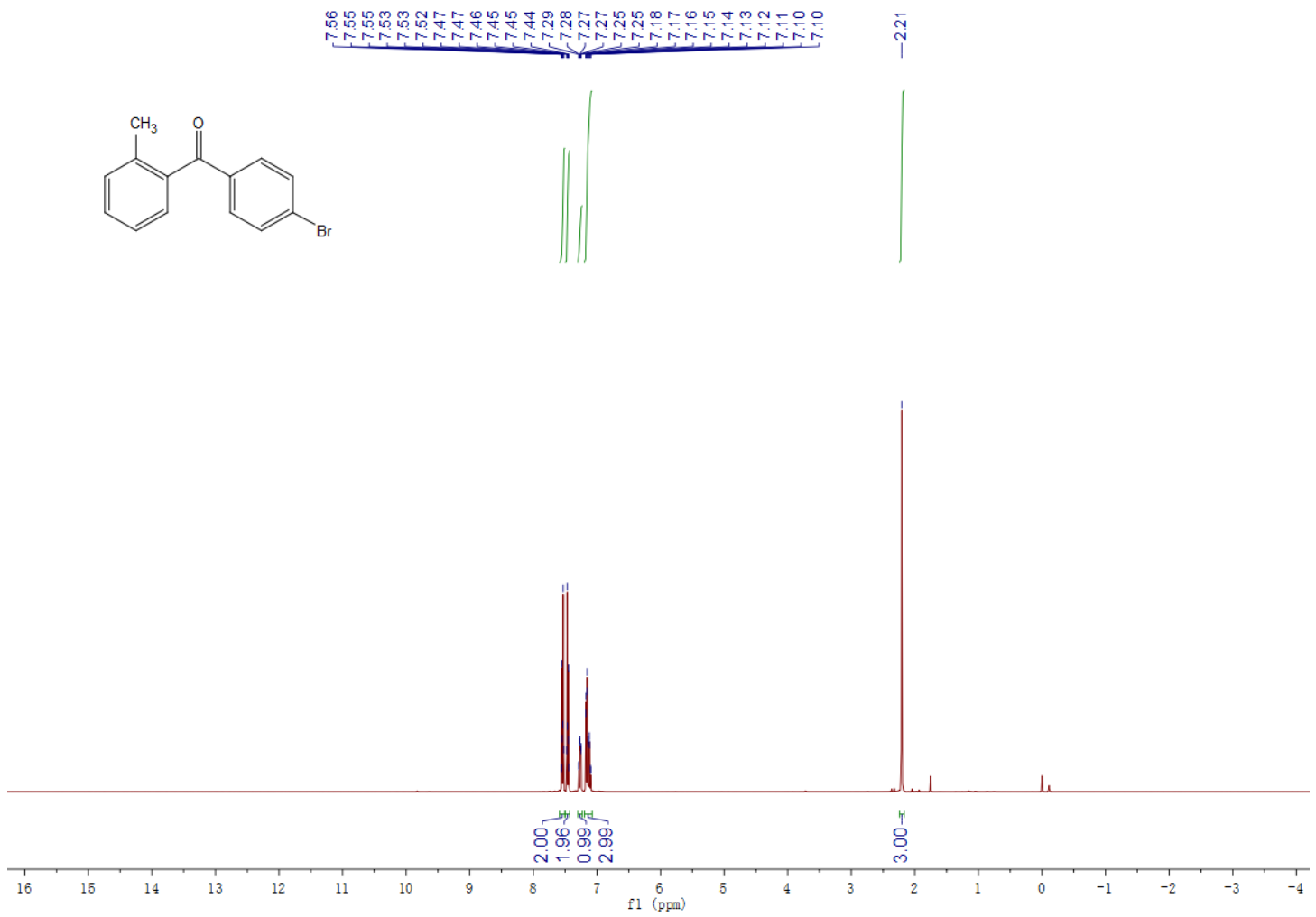

${ }^{13} \mathrm{C}$ NMR (101 MHz, $\left.\mathrm{CDCl}_{3}\right)$ of qp

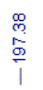

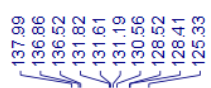

๕.
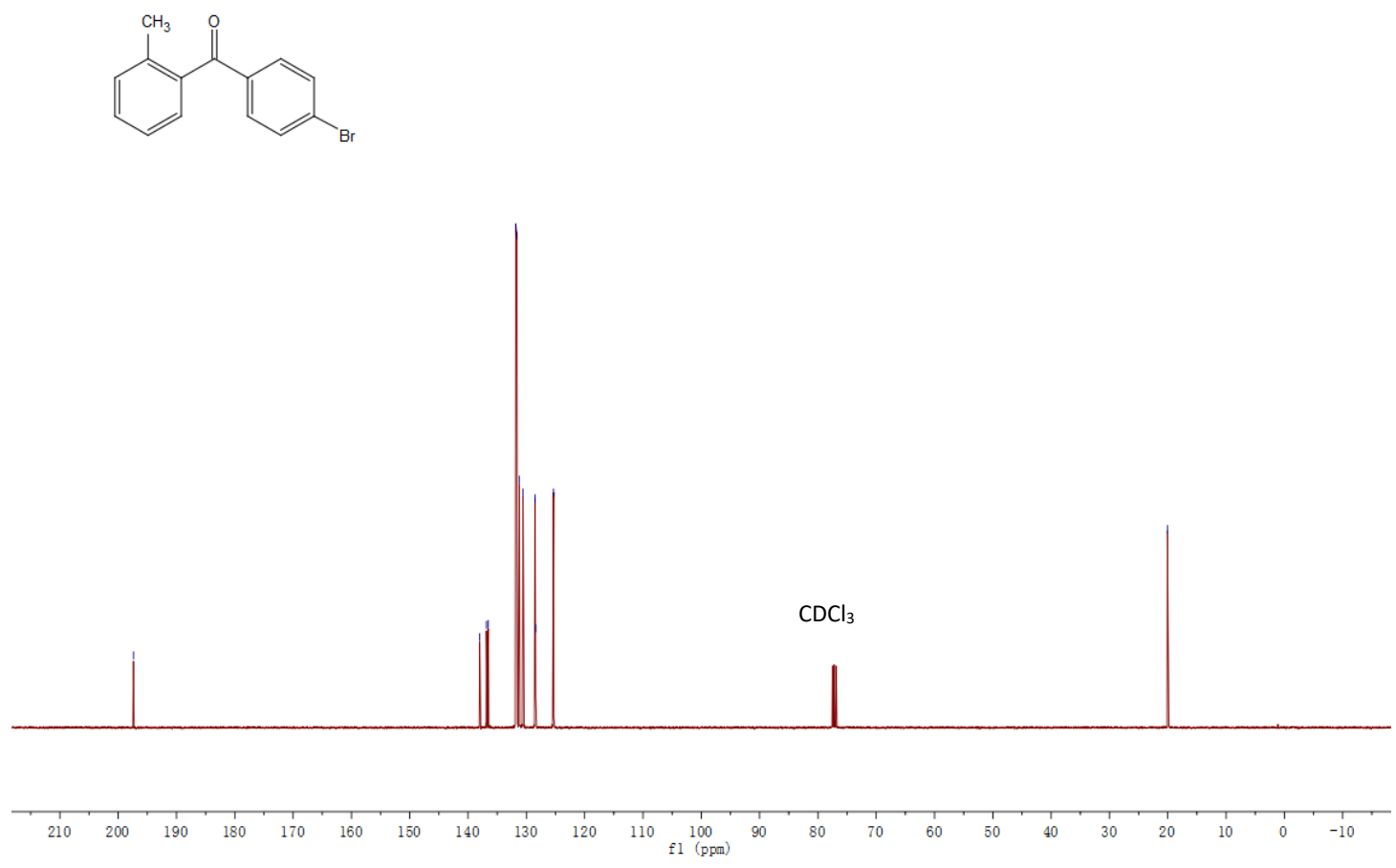

S71 
${ }^{1} \mathrm{H}$ NMR (400 MHz, $\mathrm{CDCl}_{3}$ ) of qq

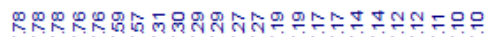
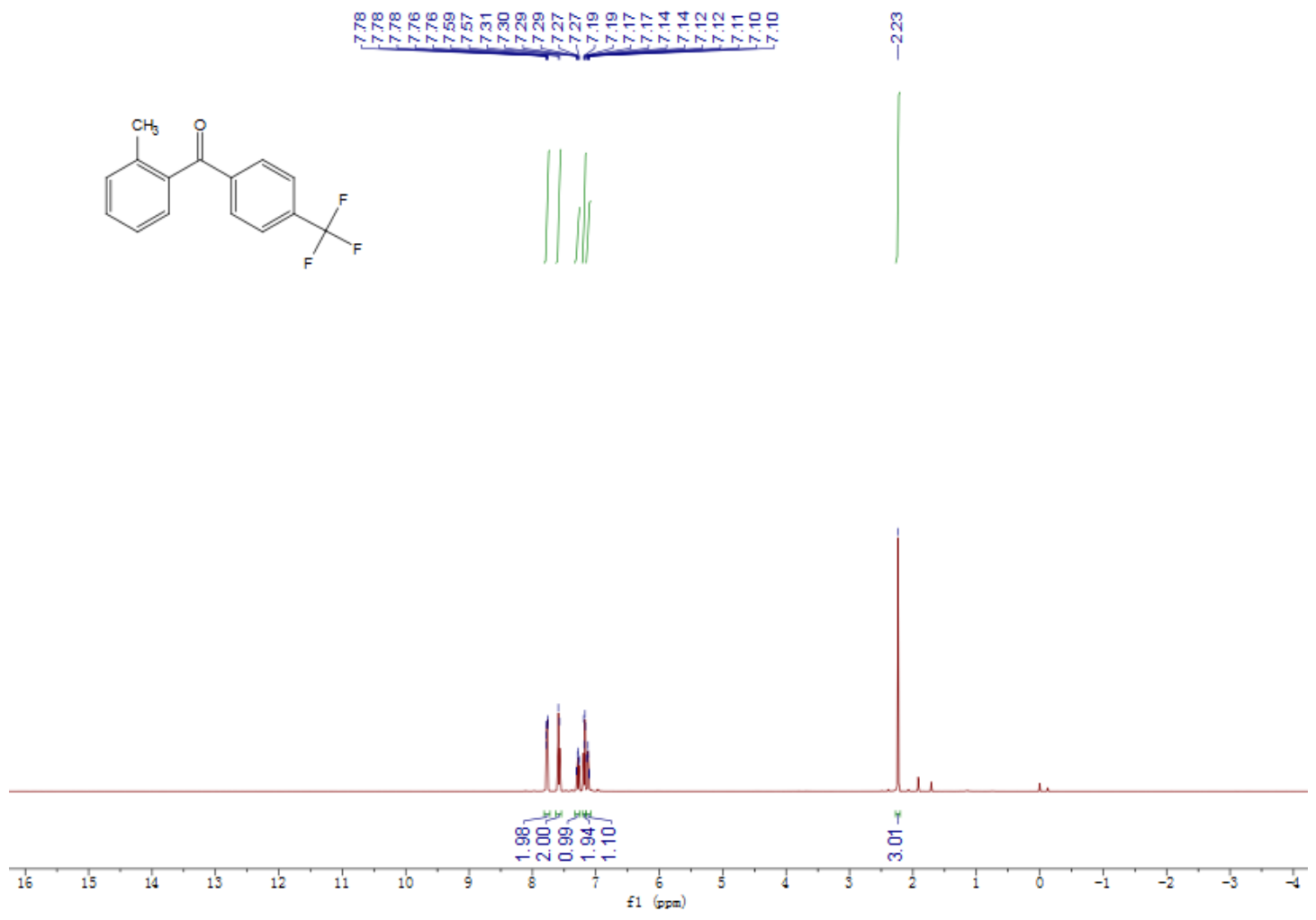

${ }^{13} \mathrm{C}$ NMR (101 MHz, $\left.\mathrm{CDCl}_{3}\right)$ of qq

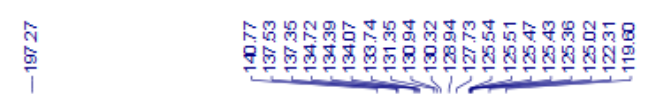

:
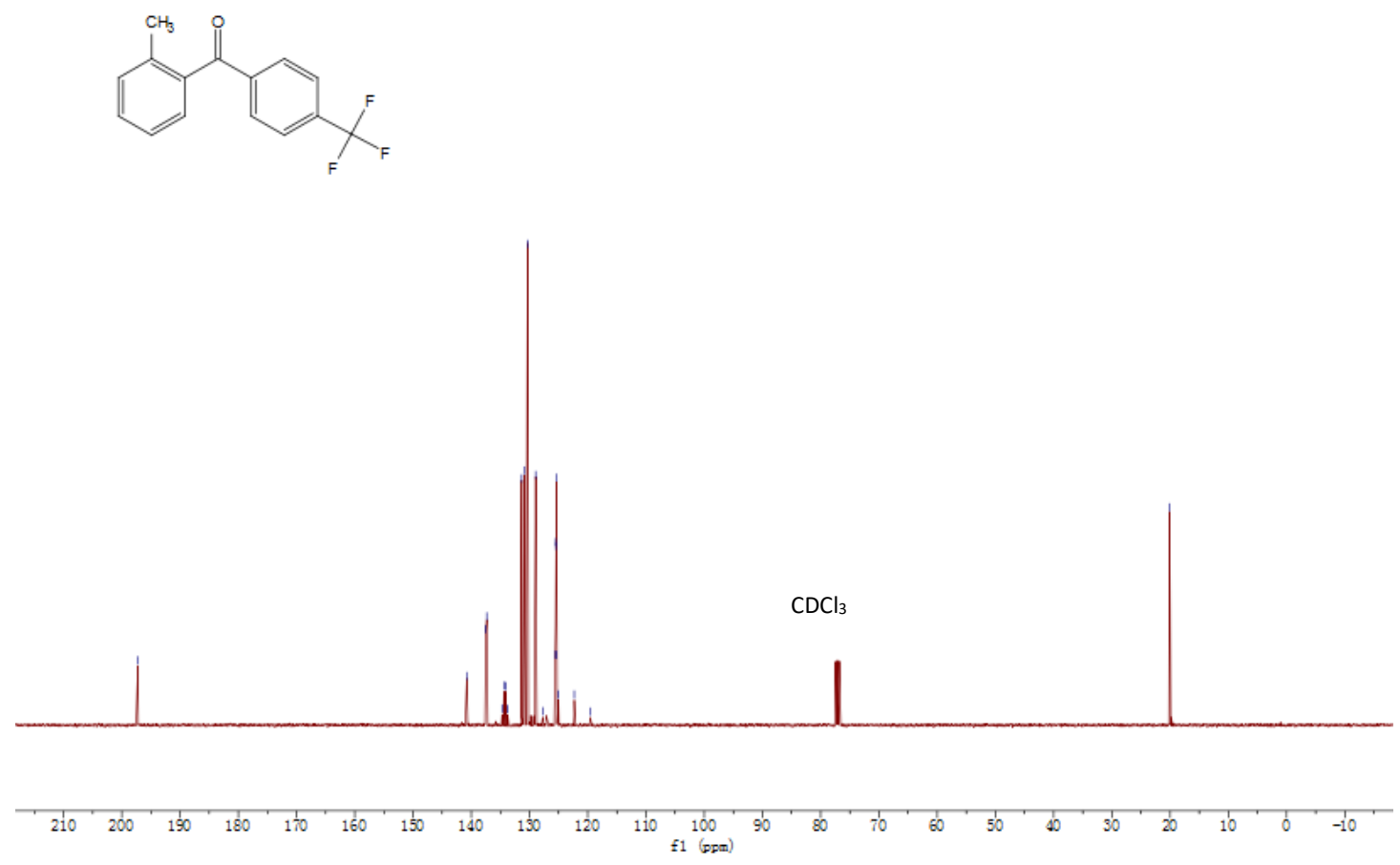

S72 
${ }^{1} \mathrm{H}$ NMR (400 MHz, $\left.\mathrm{CDCl}_{3}\right)$ of Pr

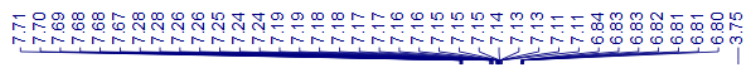

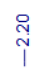
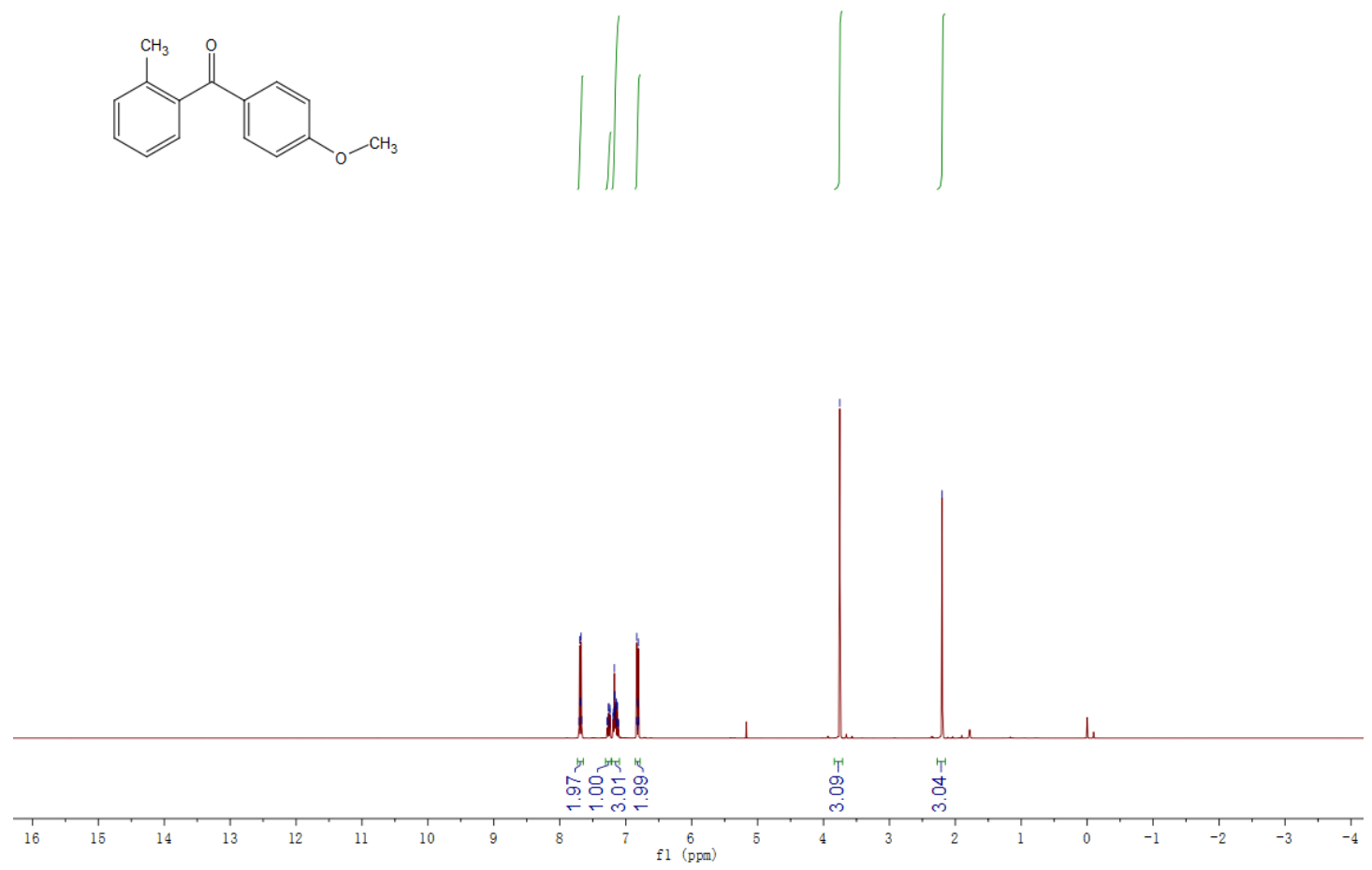

${ }^{13} \mathrm{C}$ NMR (101 MHz, $\left.\mathrm{CDCl}_{3}\right)$ of Pr
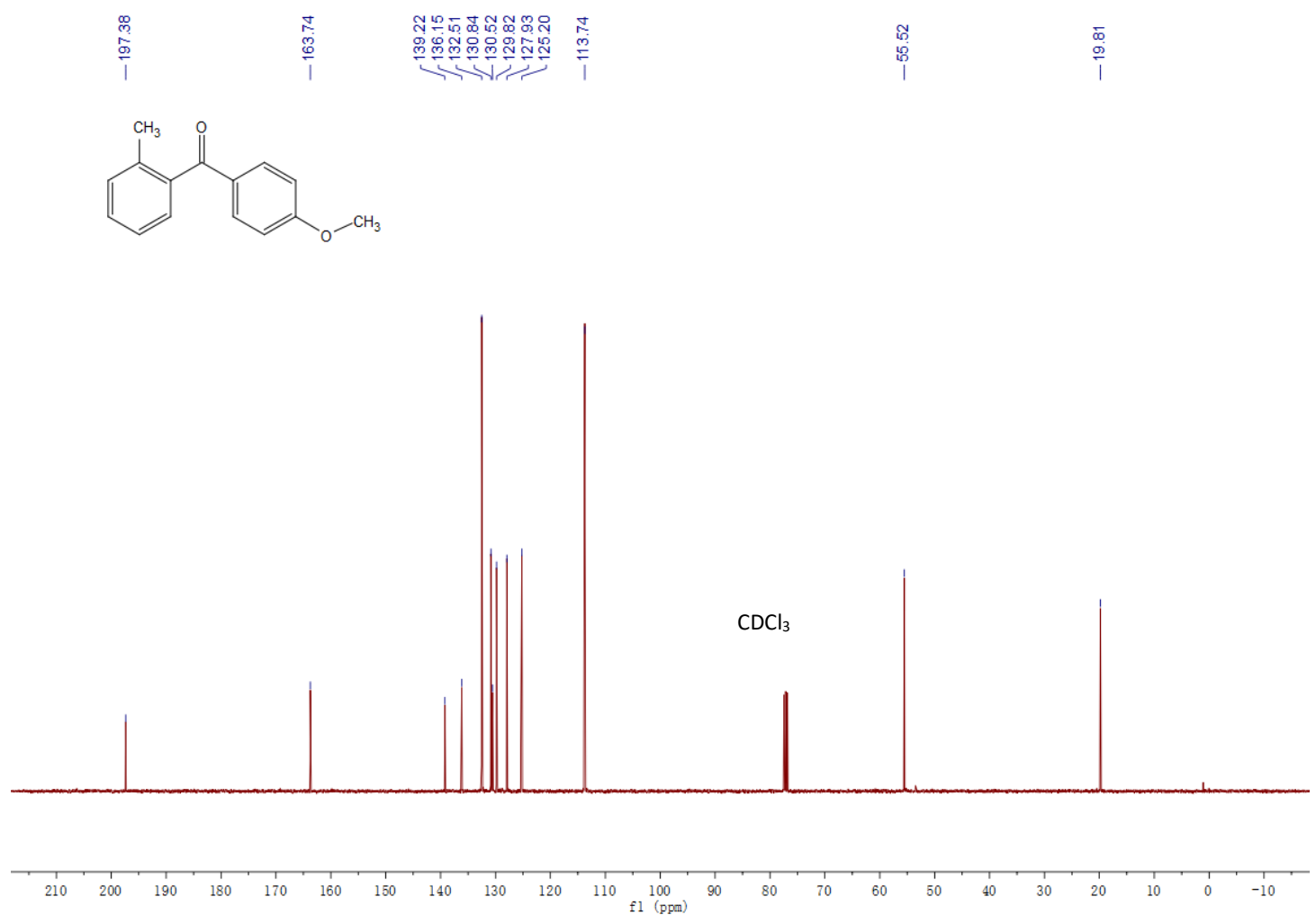

S73 
${ }^{1} \mathrm{H}$ NMR (400 MHz, $\left.\mathrm{CDCl}_{3}\right)$ of $9 \mathrm{~s}$

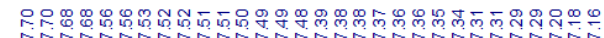
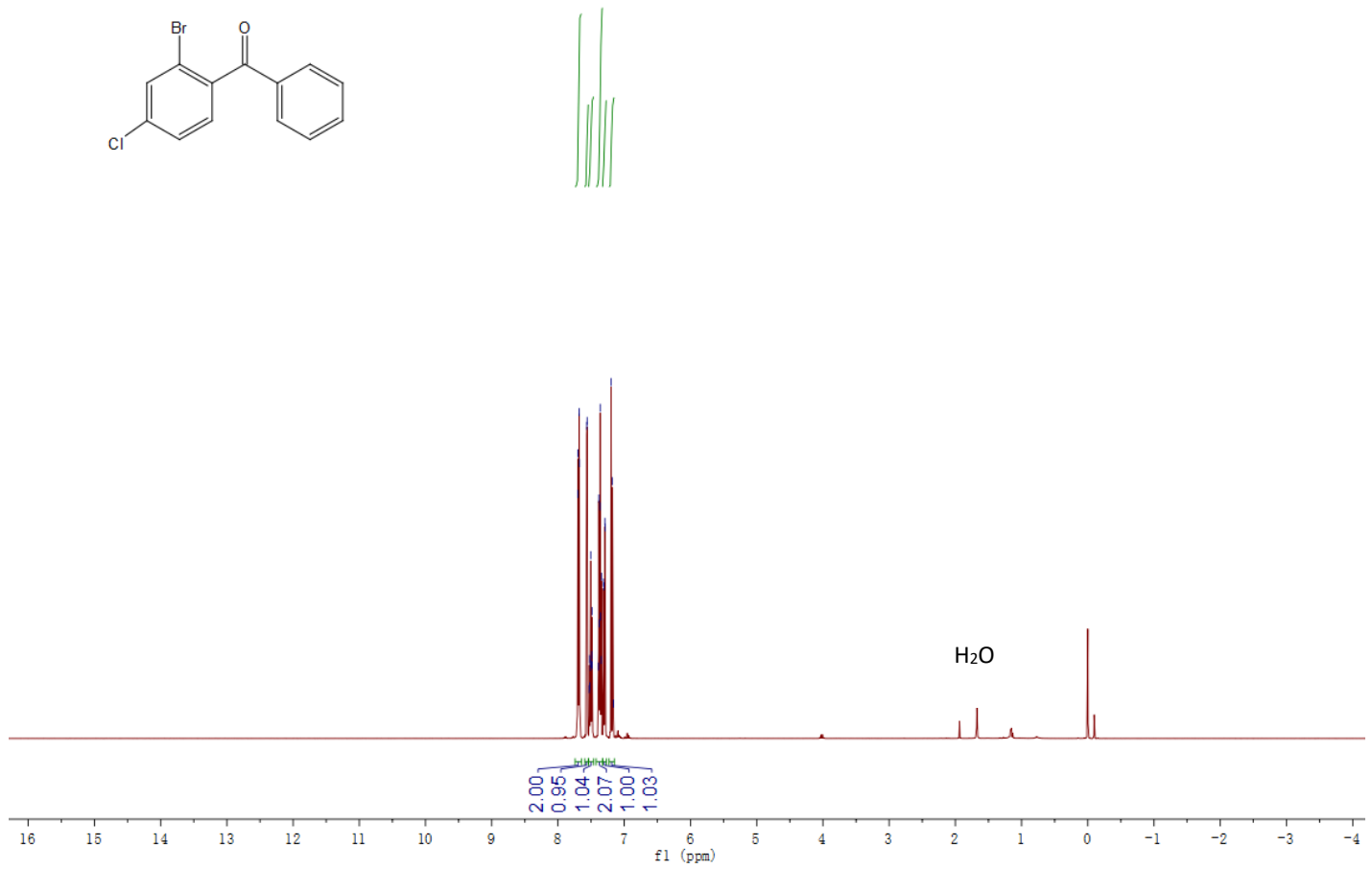

${ }^{13} \mathrm{C}$ NMR (101 MHz, $\left.\mathrm{CDCl}_{3}\right)$ of is
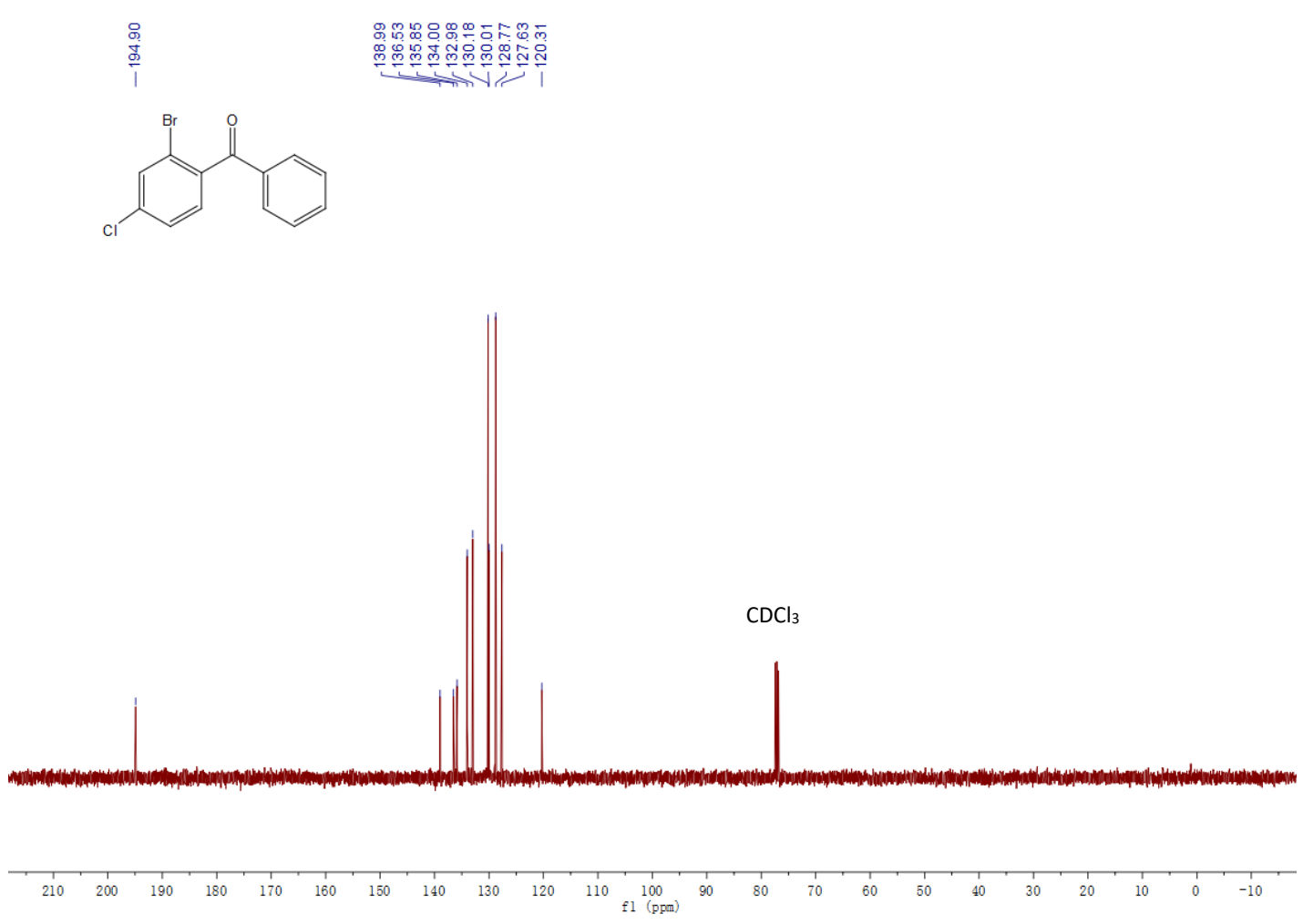

S74 
${ }^{1} \mathrm{H}$ NMR (400 MHz, $\left.\mathrm{CDCl}_{3}\right)$ of $9 \mathrm{t}$
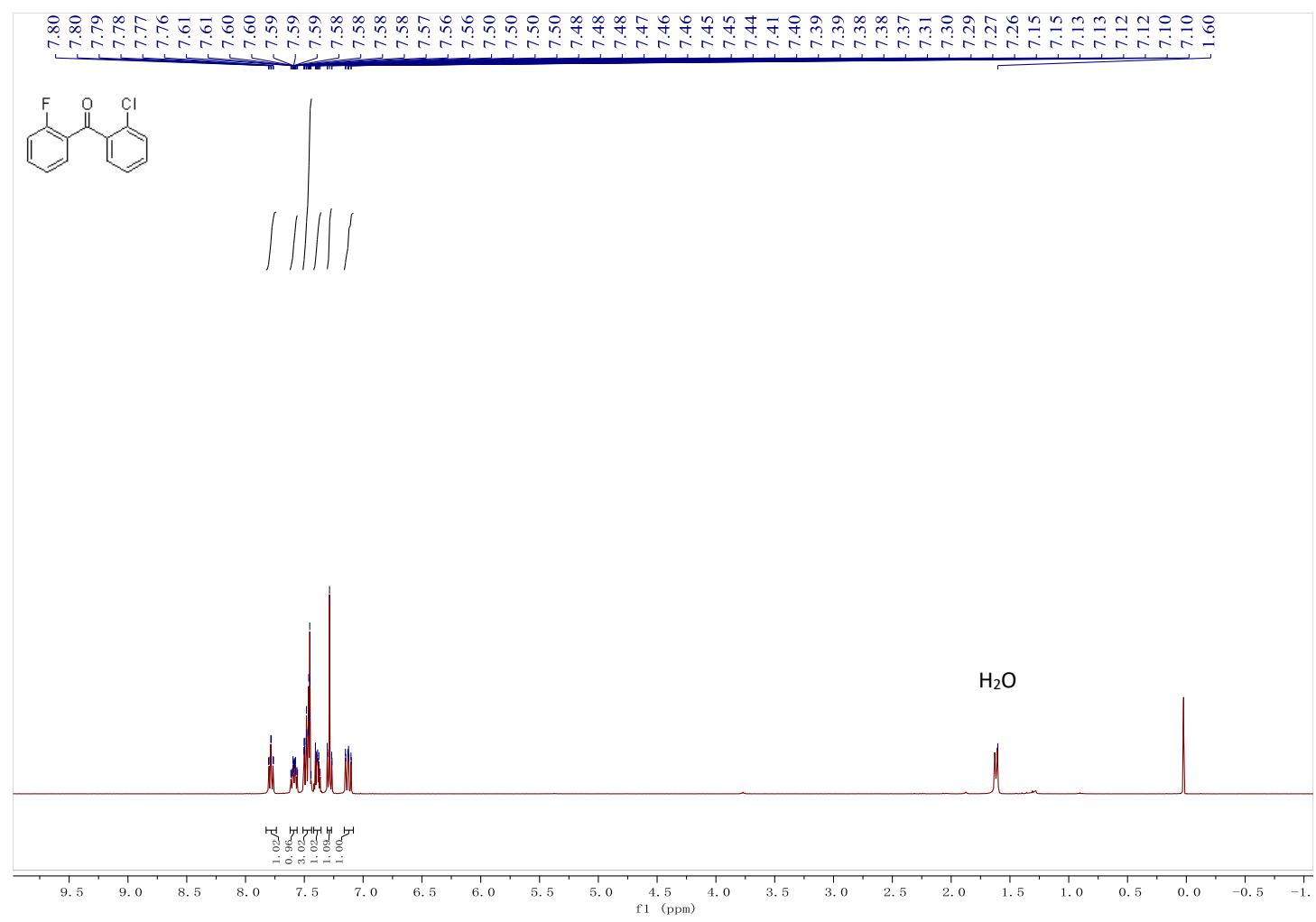

${ }^{13} \mathrm{C}$ NMR (101 MHz, $\left.\mathrm{CDCl}_{3}\right)$ of 9t

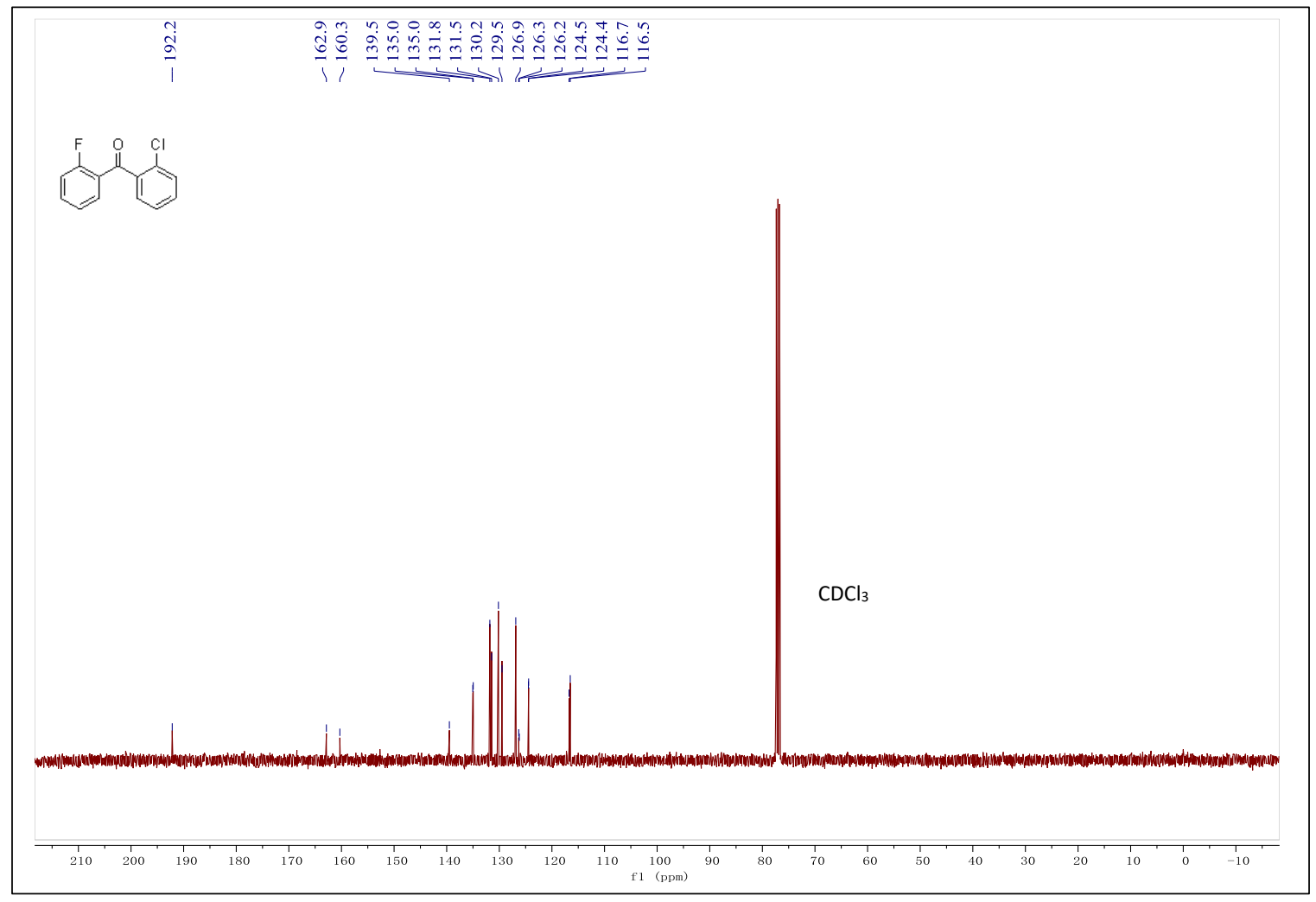


${ }^{1} \mathrm{H}$ NMR (600 MHz, CDCl 3$)$ of $9 u$

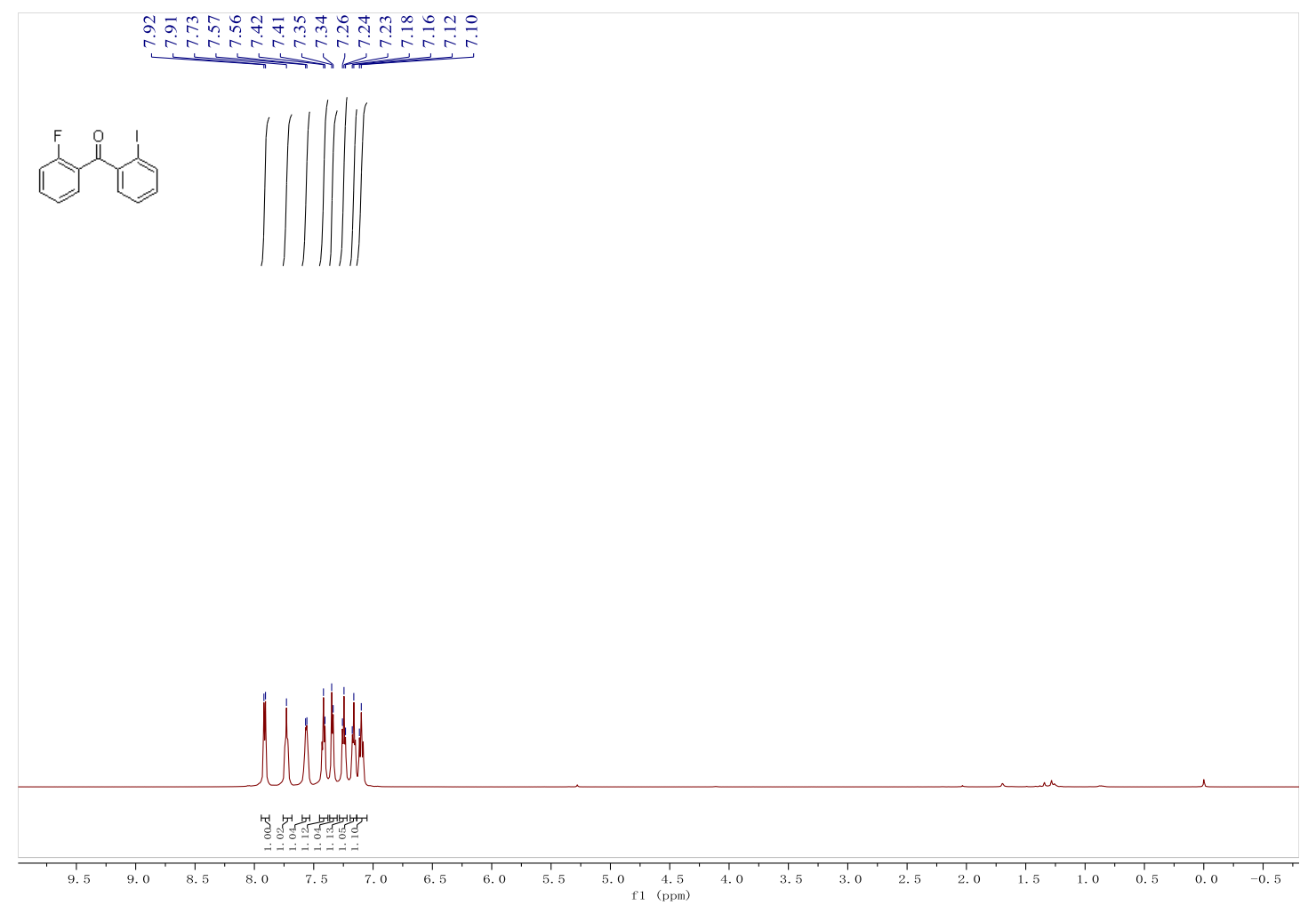

${ }^{13} \mathrm{C}$ NMR (151 MHz, CDCl 3$)$ of 9u
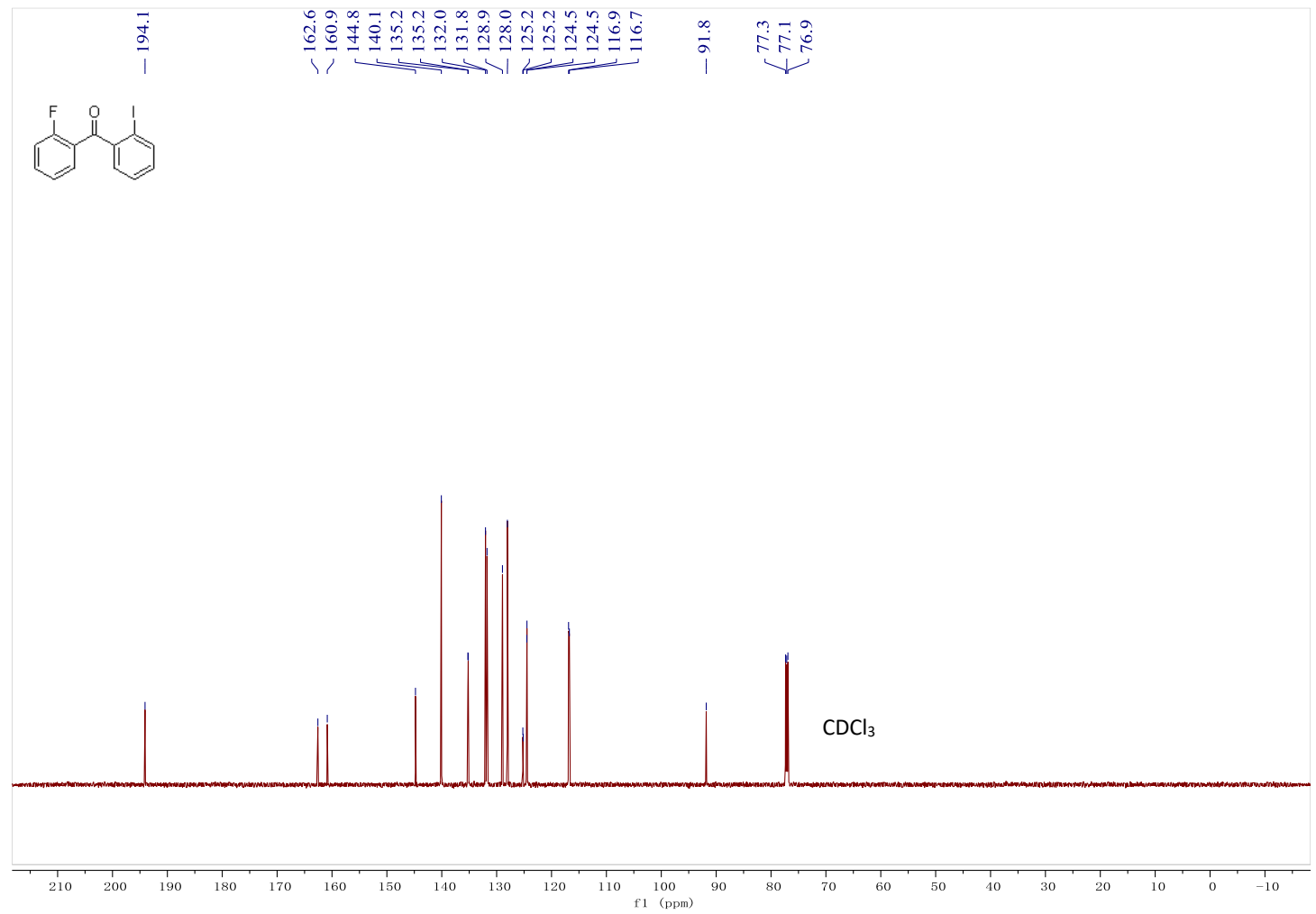
${ }^{1} \mathrm{H}$ NMR (600 MHz, CDCl 3$)$ of 9v

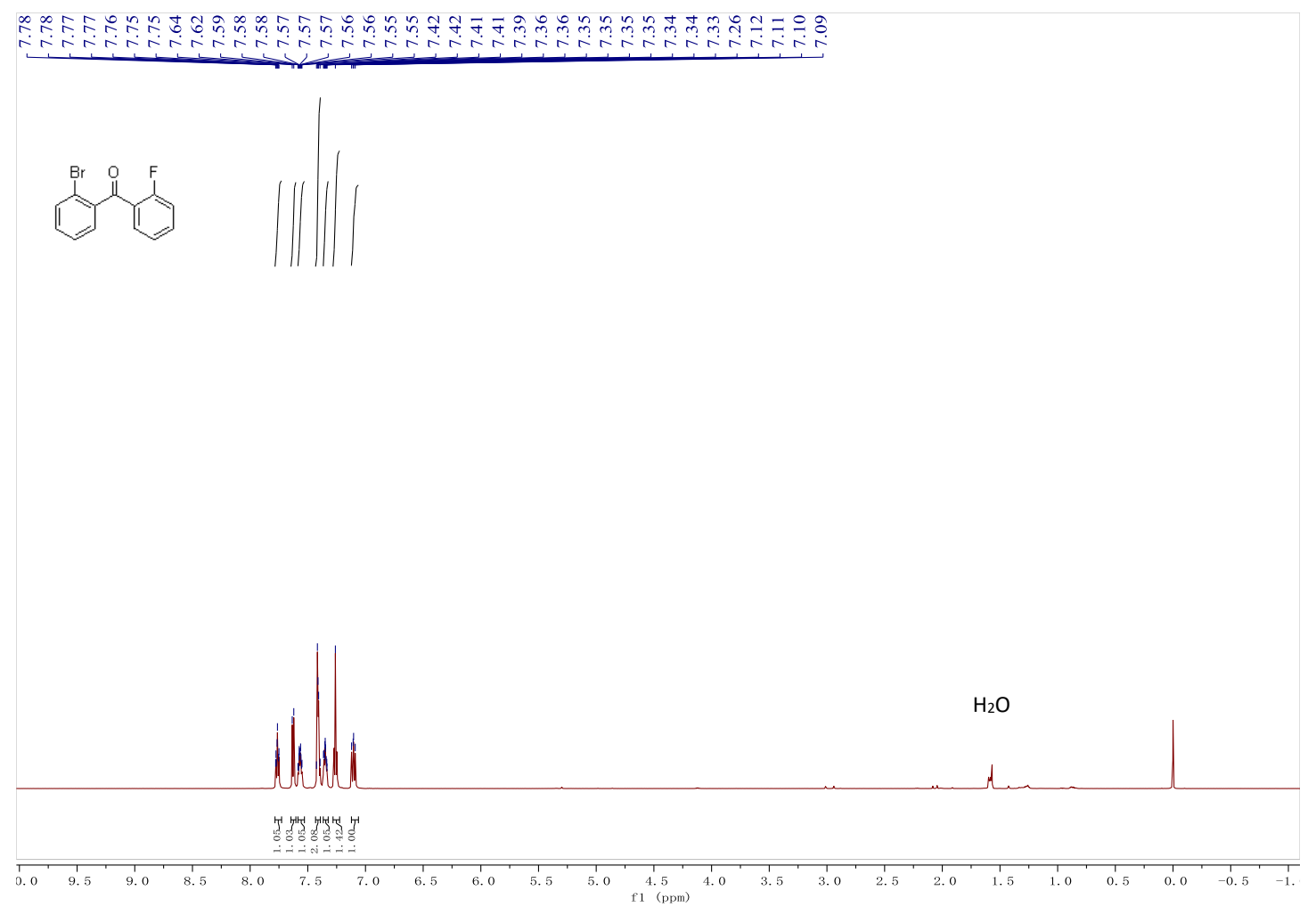

${ }^{13} \mathrm{C}$ NMR (101 MHz, CDCl 3$)$ of 9v
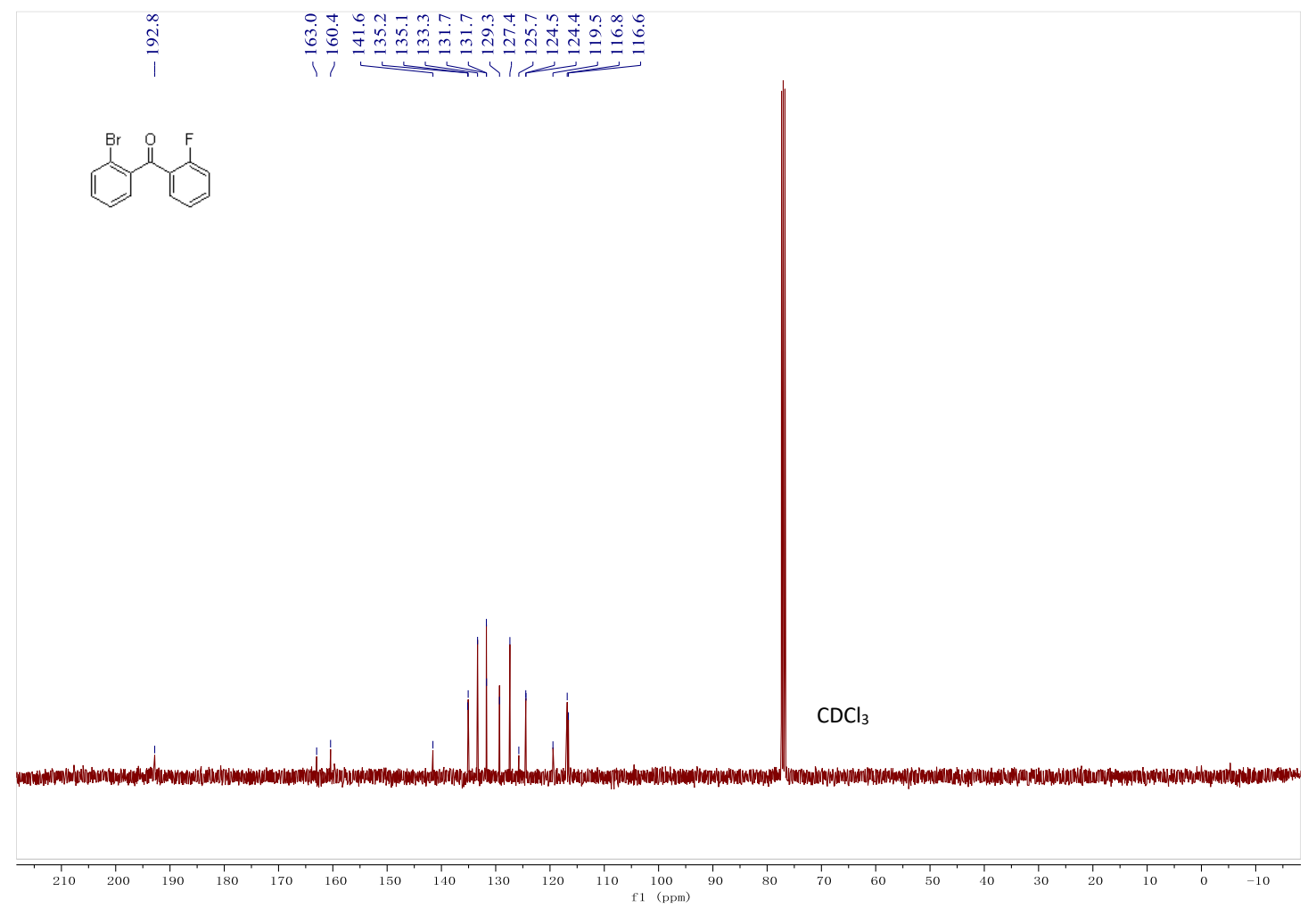
${ }^{1} \mathrm{H}$ NMR (400 MHz, $\left.\mathrm{CDCl}_{3}\right)$ of 9w

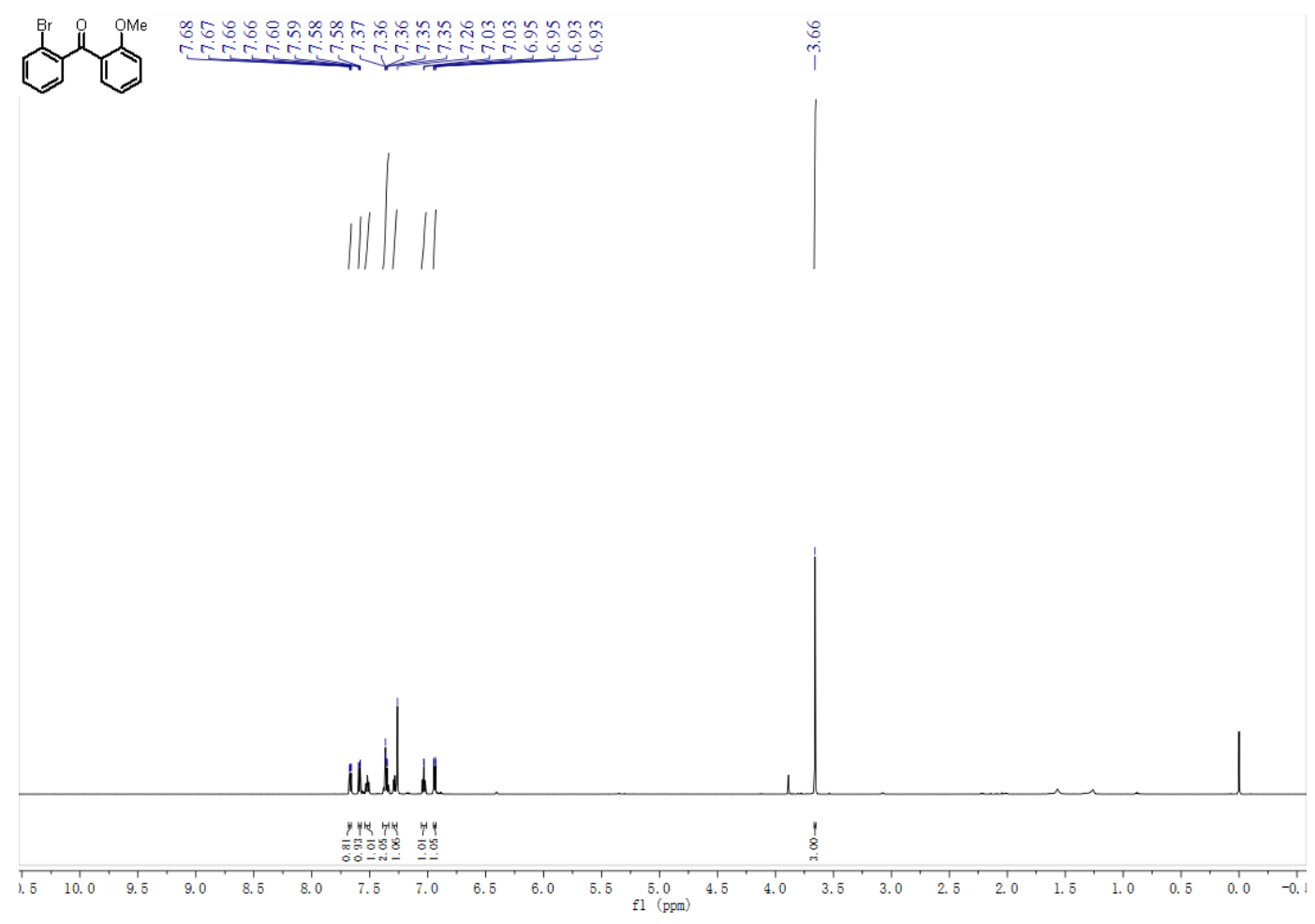

${ }^{13} \mathrm{C}$ NMR (101 MHz, $\left.\mathrm{CDCl}_{3}\right)$ of 9w
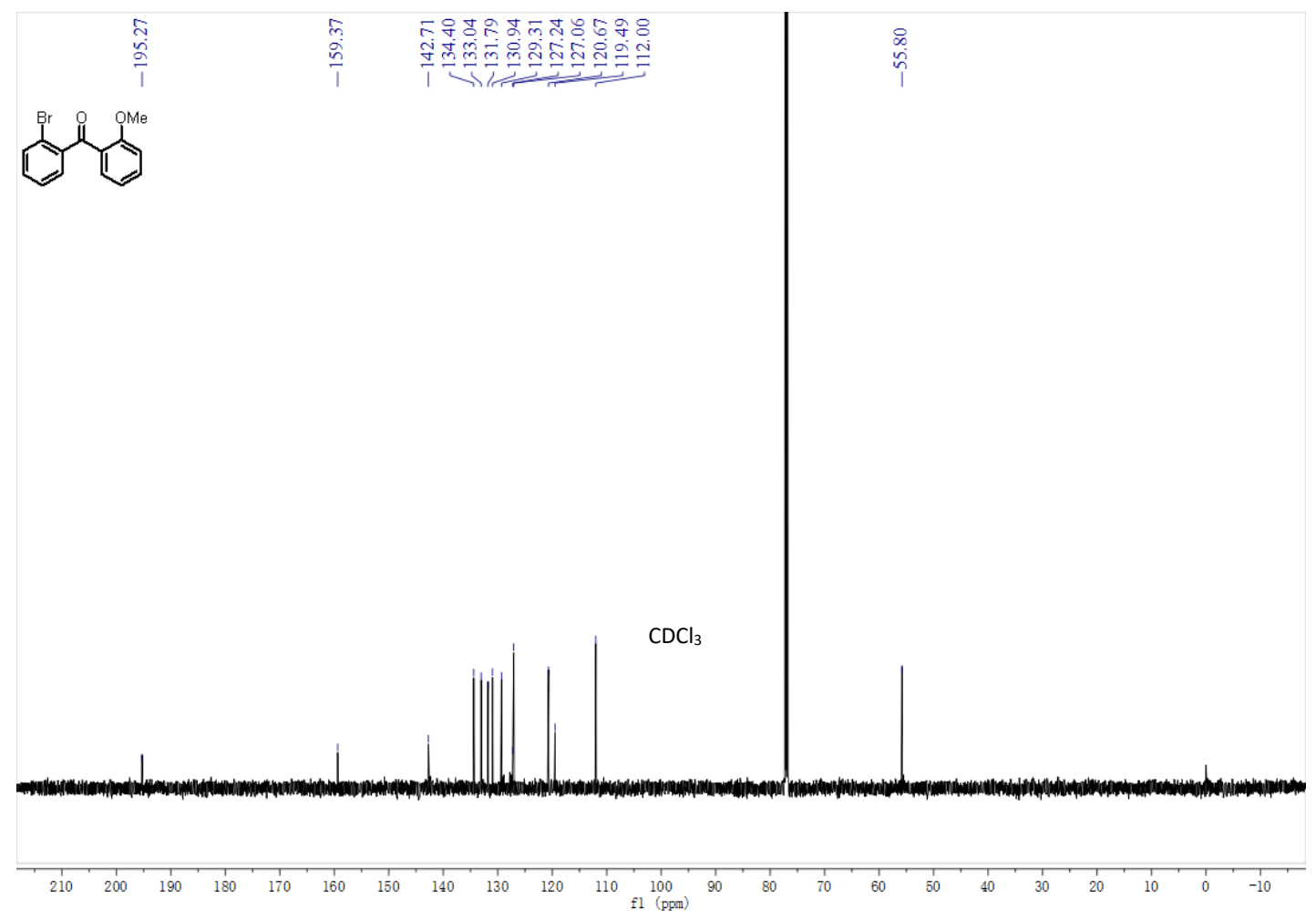
${ }^{1} \mathrm{H}$ NMR (400 MHz, $\left.\mathrm{CDCl}_{3}\right)$ of $10 \mathrm{a}$

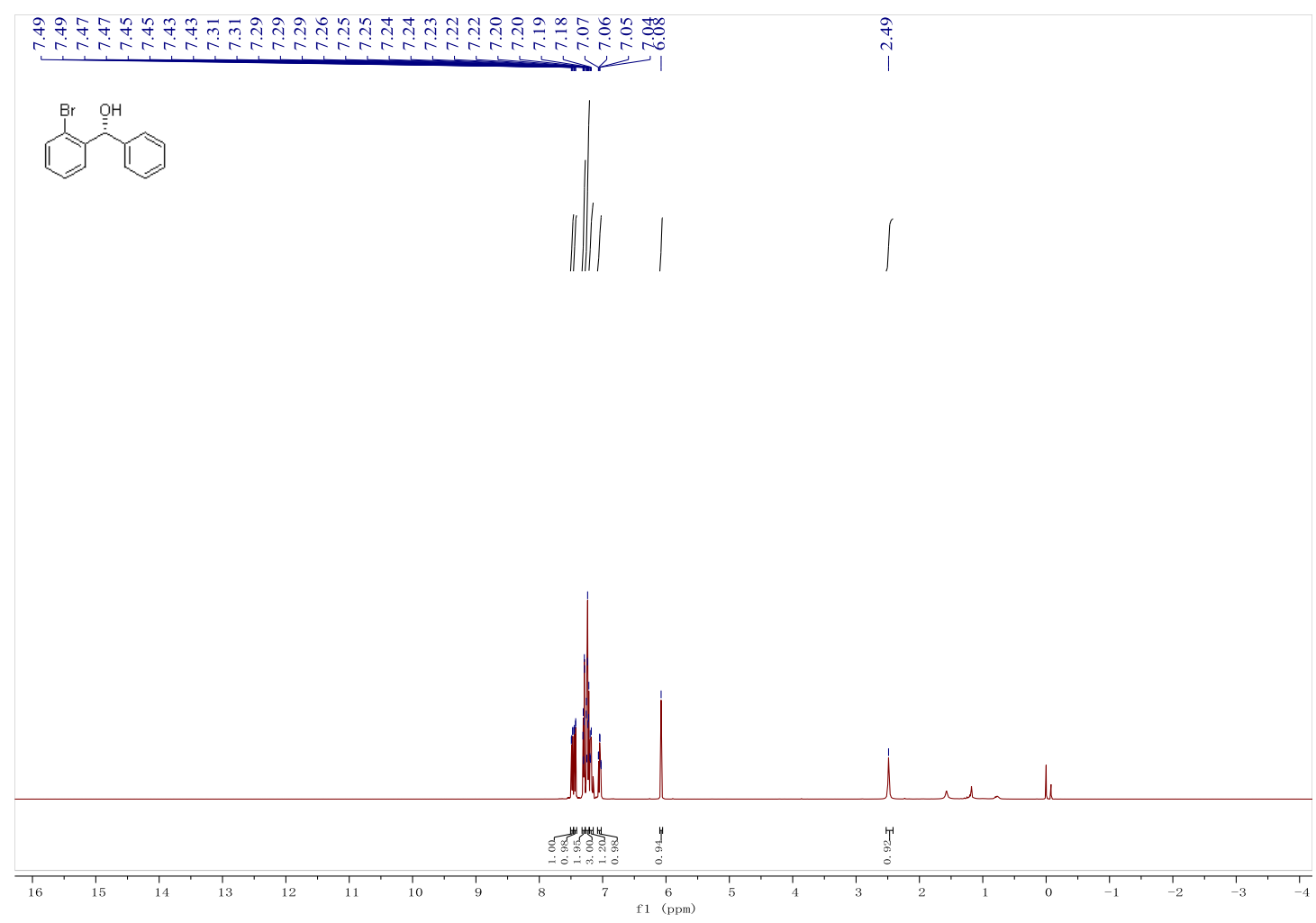

${ }^{13} \mathrm{C}$ NMR (101 MHz, CDCl3) of 10a

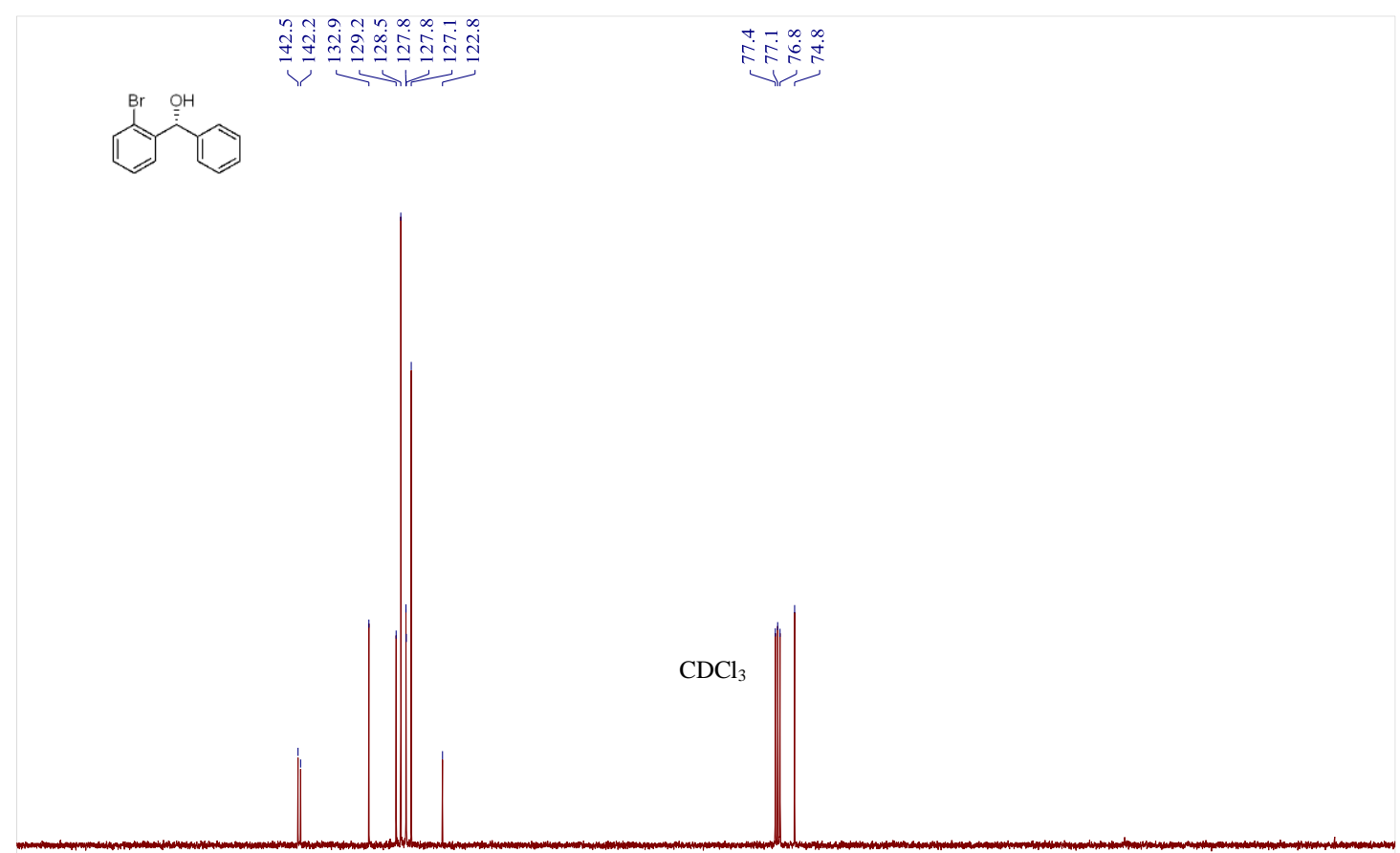

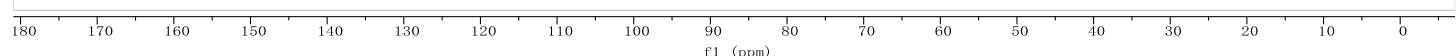


${ }^{1} \mathrm{H}$ NMR (400 $\left.\mathrm{MHz}, \mathrm{CDCl}_{3}\right)$ of $10 \mathrm{~b}$

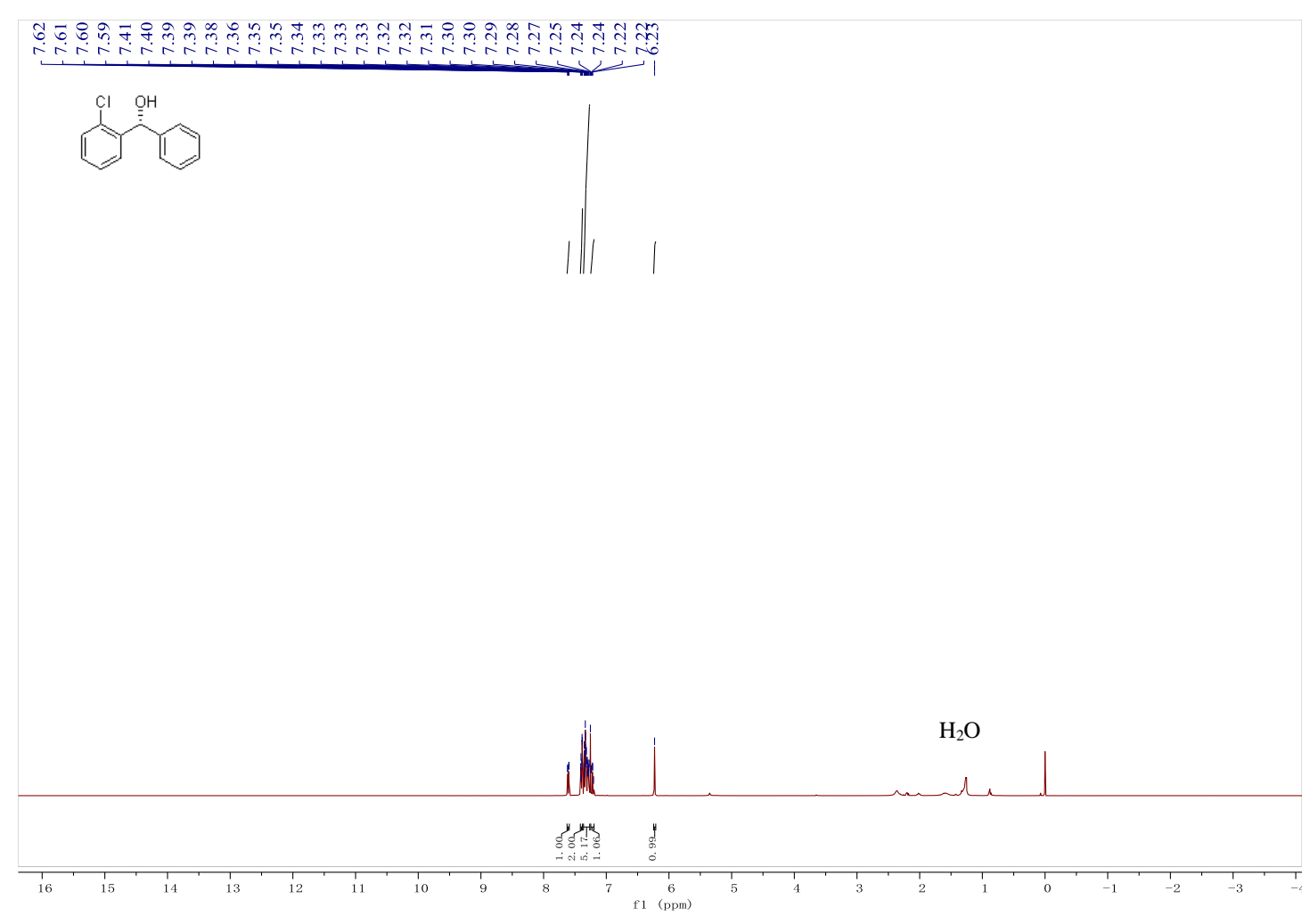

${ }^{13} \mathrm{C}$ NMR (101 MHz, $\left.\mathrm{CDCl}_{3}\right)$ of 10b

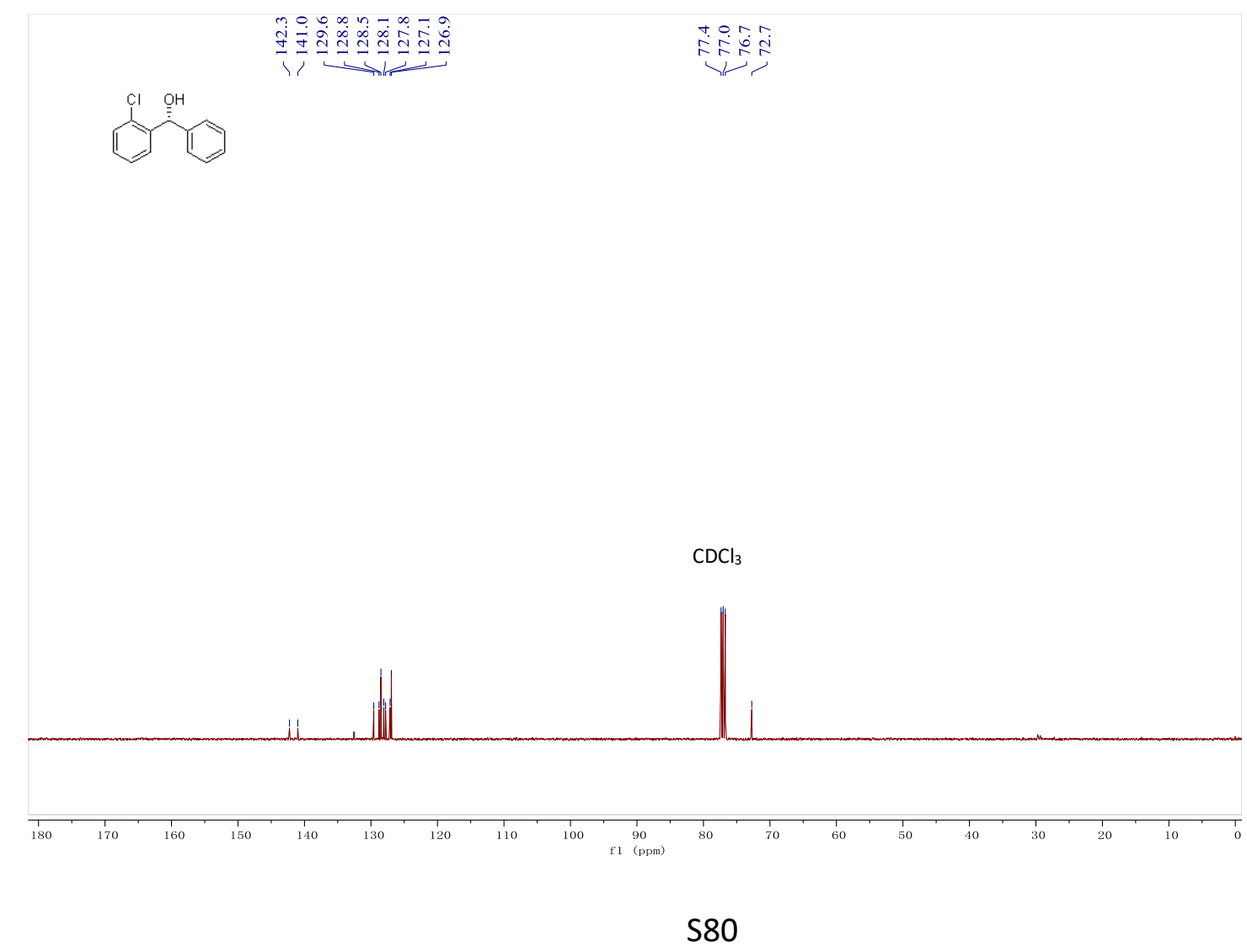


${ }^{1} \mathrm{H}$ NMR (400 $\left.\mathrm{MHz}, \mathrm{CDCl}_{3}\right)$ of $10 \mathrm{c}$
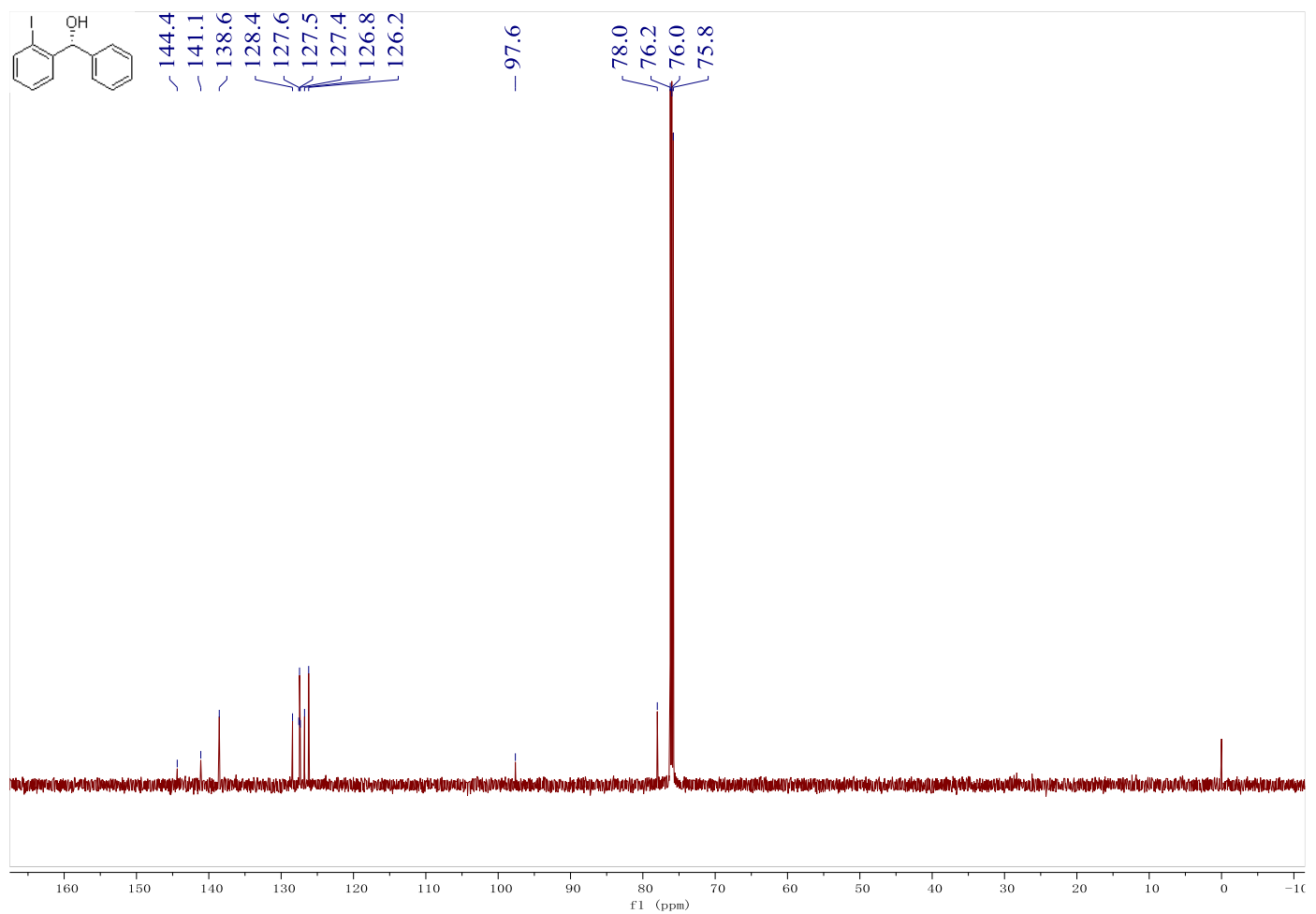

${ }^{1} \mathrm{H}$ NMR (400 MHz, $\left.\mathrm{CDCl}_{3}\right)$ of $10 \mathrm{c}$

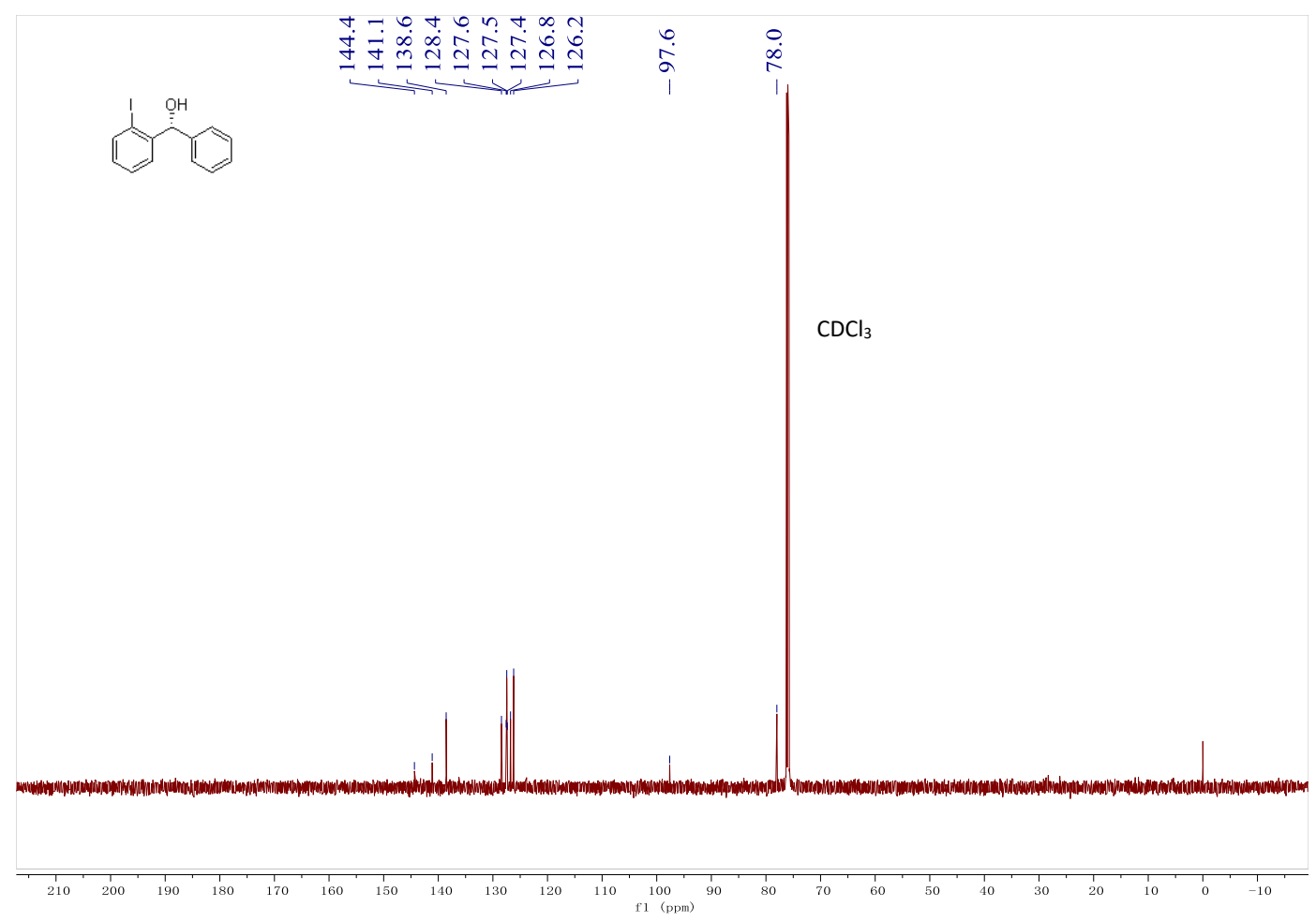


${ }^{1} \mathrm{H}$ NMR (400 MHz, CDCl 3$)$ of $10 d$

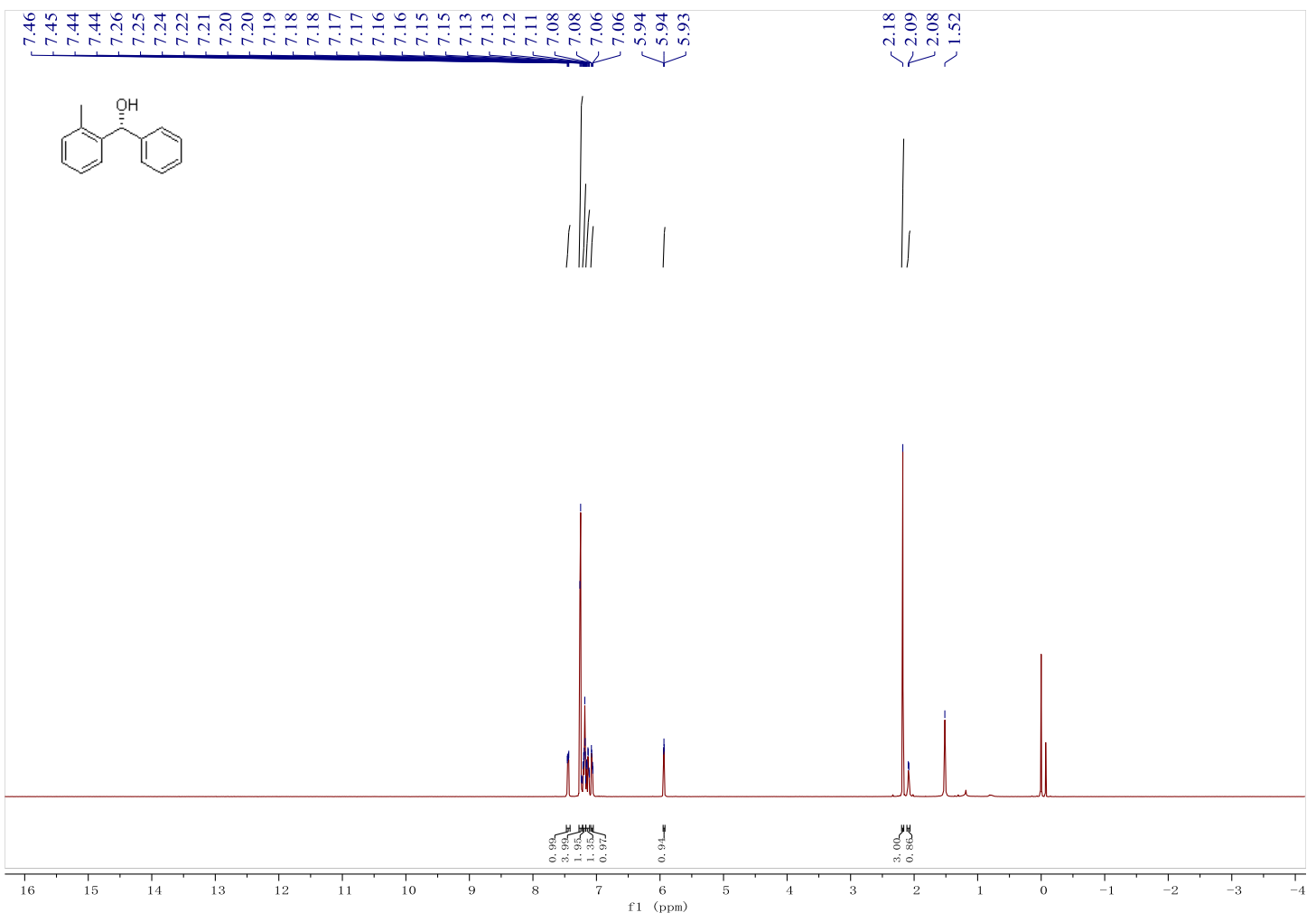

${ }^{13} \mathrm{C}$ NMR (101 MHz, $\left.\mathrm{CDCl}_{3}\right)$ of 10d

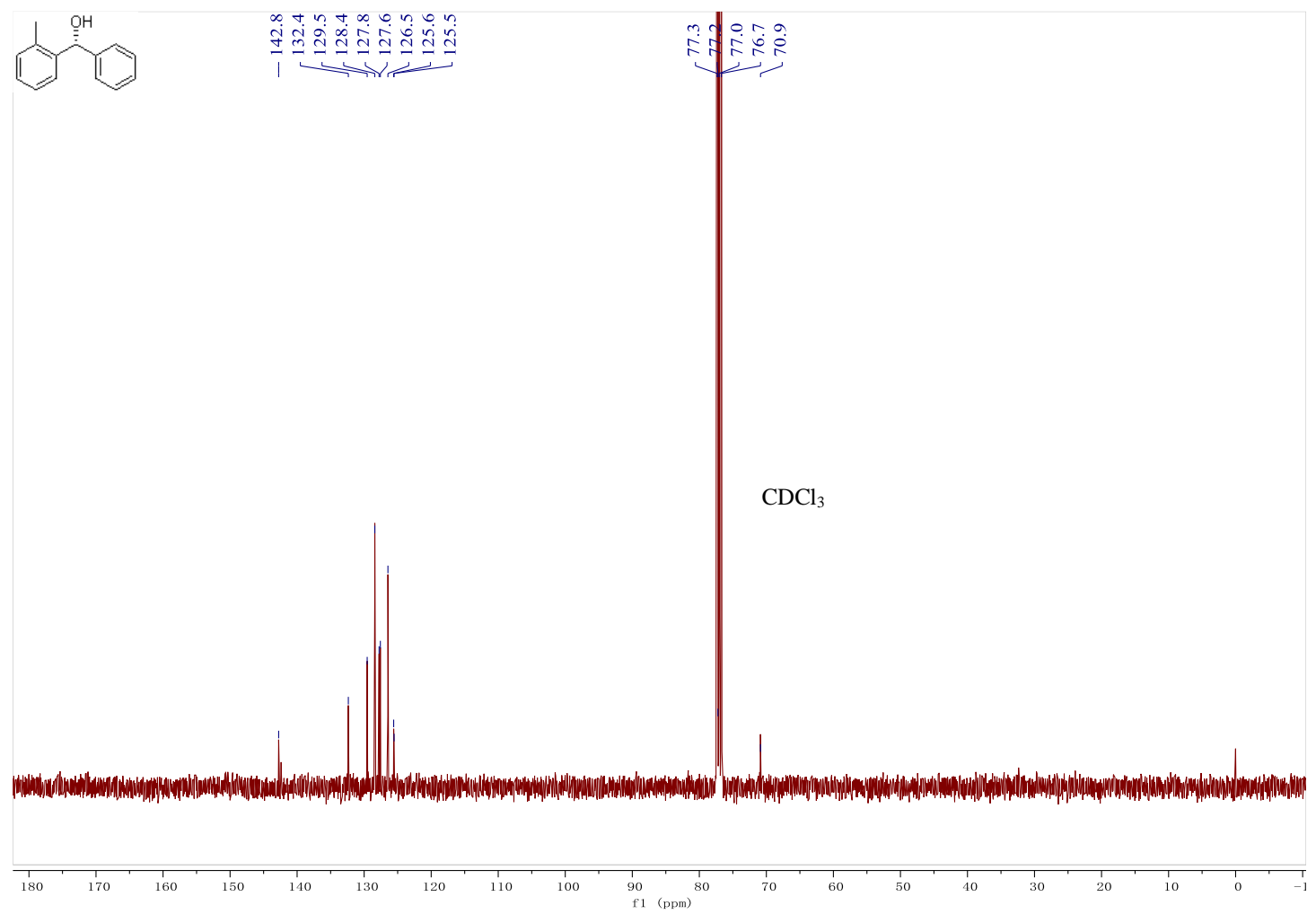

S82 
${ }^{1} \mathrm{H}$ NMR (400 MHz, $\left.\mathrm{CDCl}_{3}\right)$ of $10 \mathrm{e}$

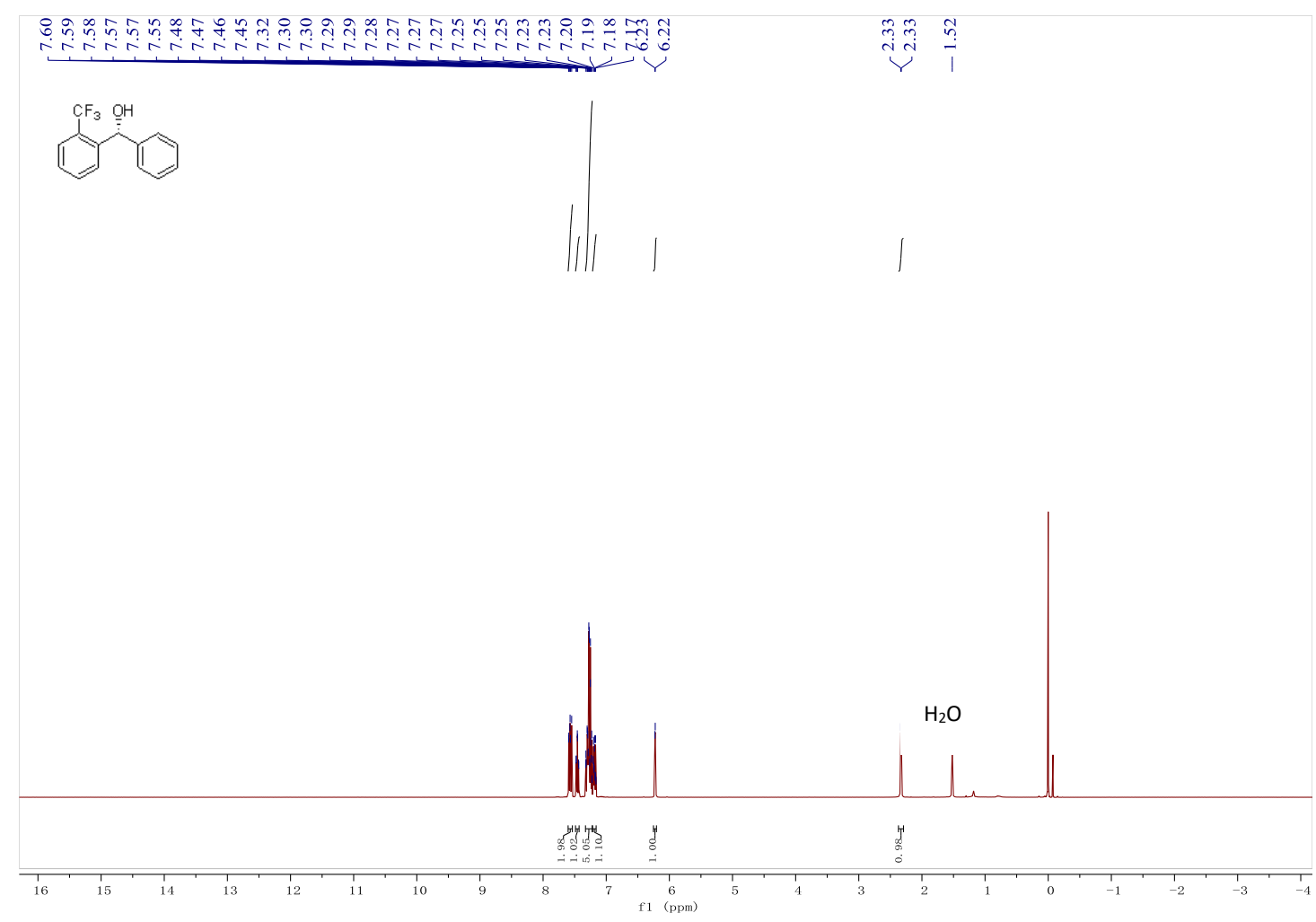

${ }^{13} \mathrm{C}$ NMR (101 MHz, CDCl 3$)$ of 10e
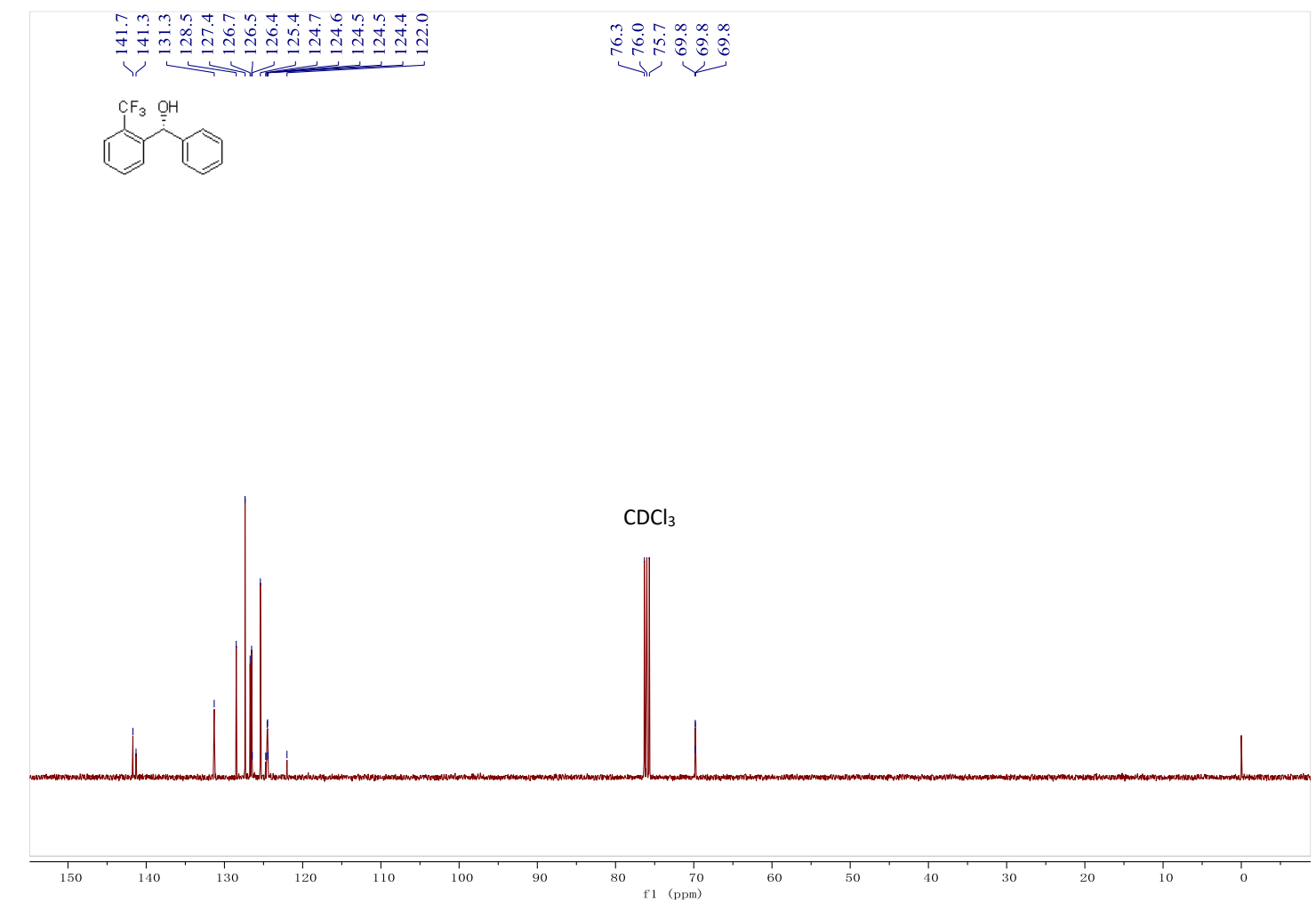
${ }^{19} \mathrm{~F}$ NMR (376 MHz, $\left.\mathrm{CDCl}_{3}\right)$ of 10e

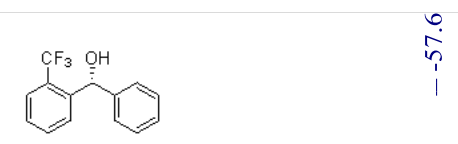

$\mathrm{CDCl}_{3}$

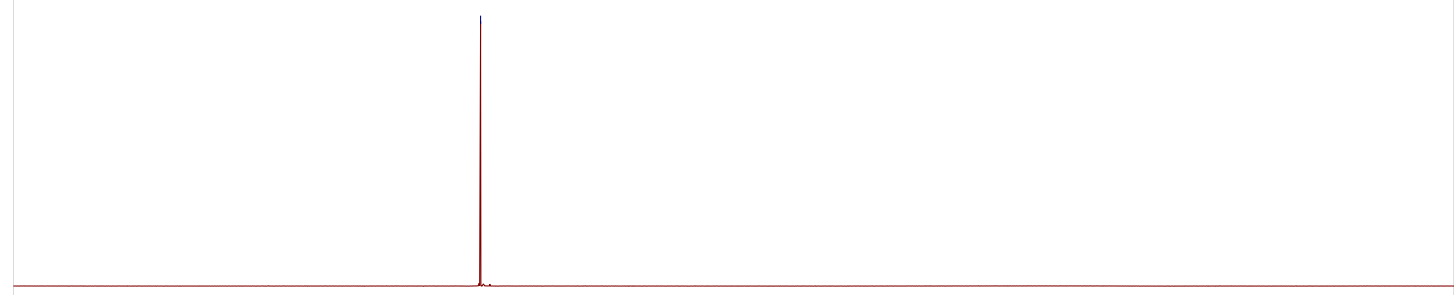

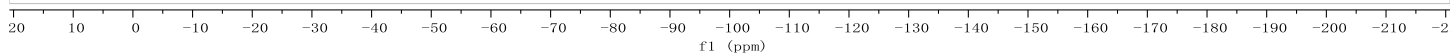


$1 \mathrm{H}$ NMR (400 MHz, CDCl3) of $10 \mathrm{f}$

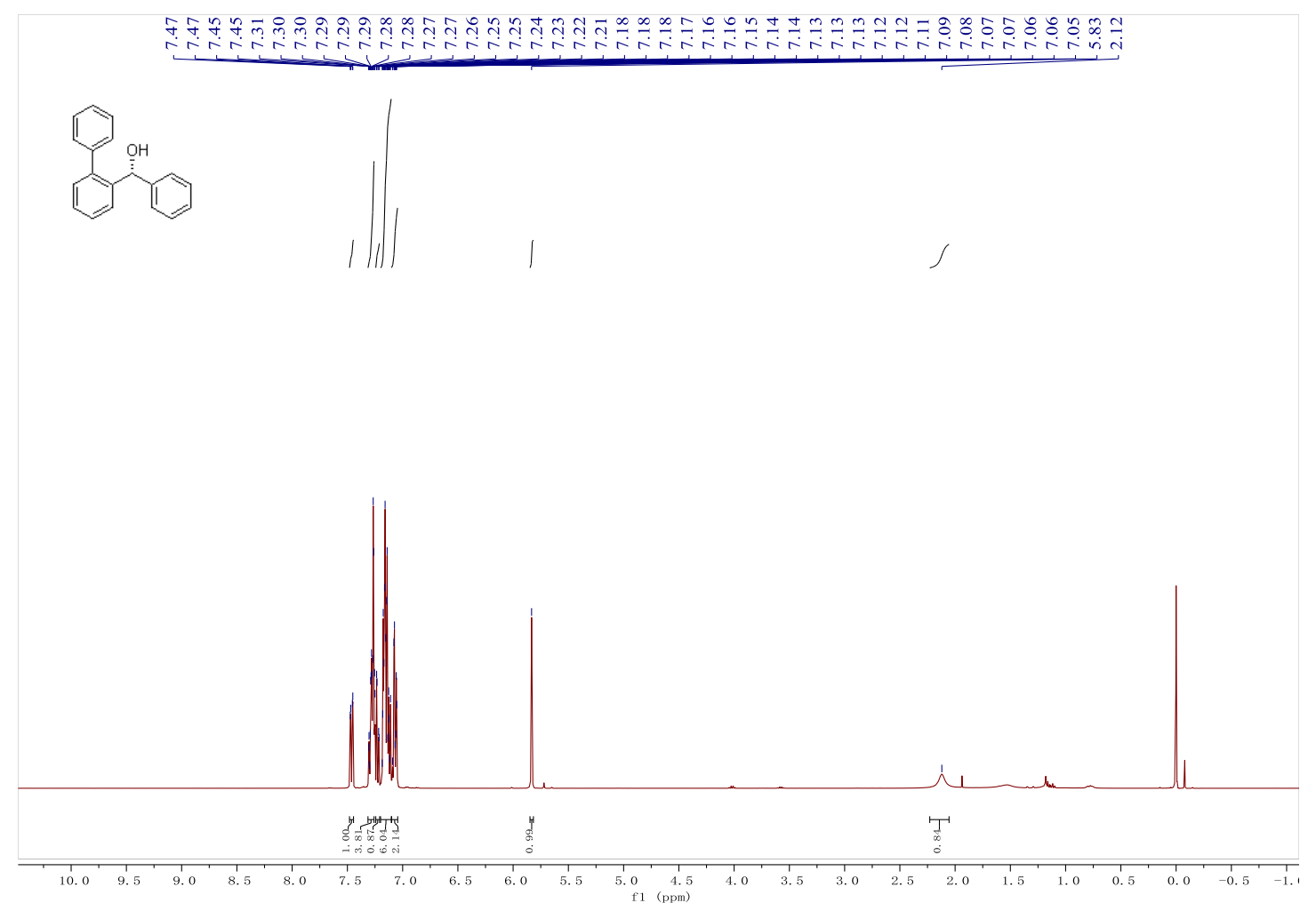

${ }^{13} \mathrm{C}$ NMR (101 MHz, $\left.\mathrm{CDCl}_{3}\right)$ of $10 \mathrm{f}$

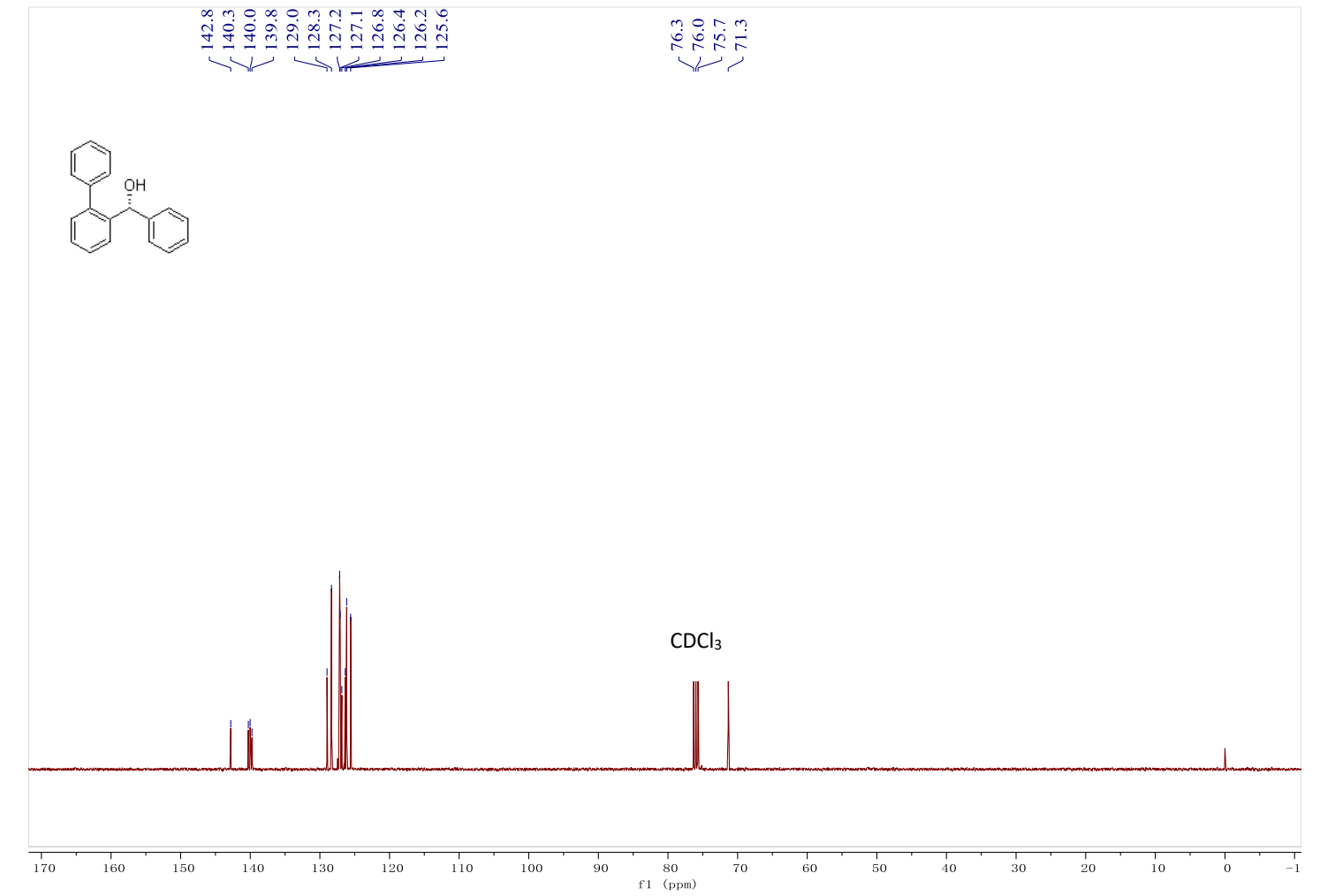


${ }^{1} \mathrm{H}$ NMR (400 MHz, $\left.\mathrm{CDCl}_{3}\right)$ of $10 \mathrm{~g}$

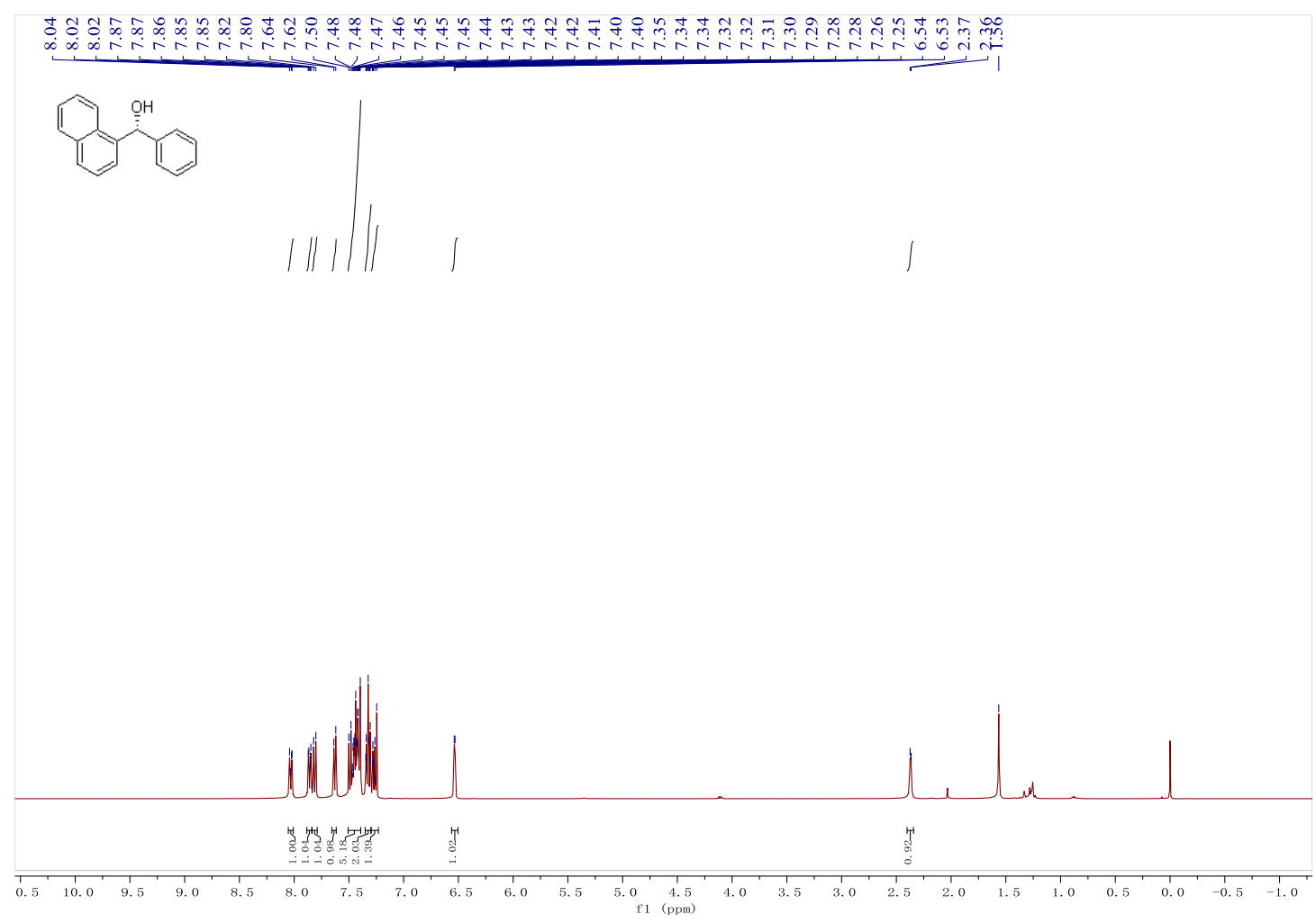

${ }^{13} \mathrm{C}$ NMR (101 MHz, $\left.\mathrm{CDCl}_{3}\right)$ of $10 \mathrm{~g}$
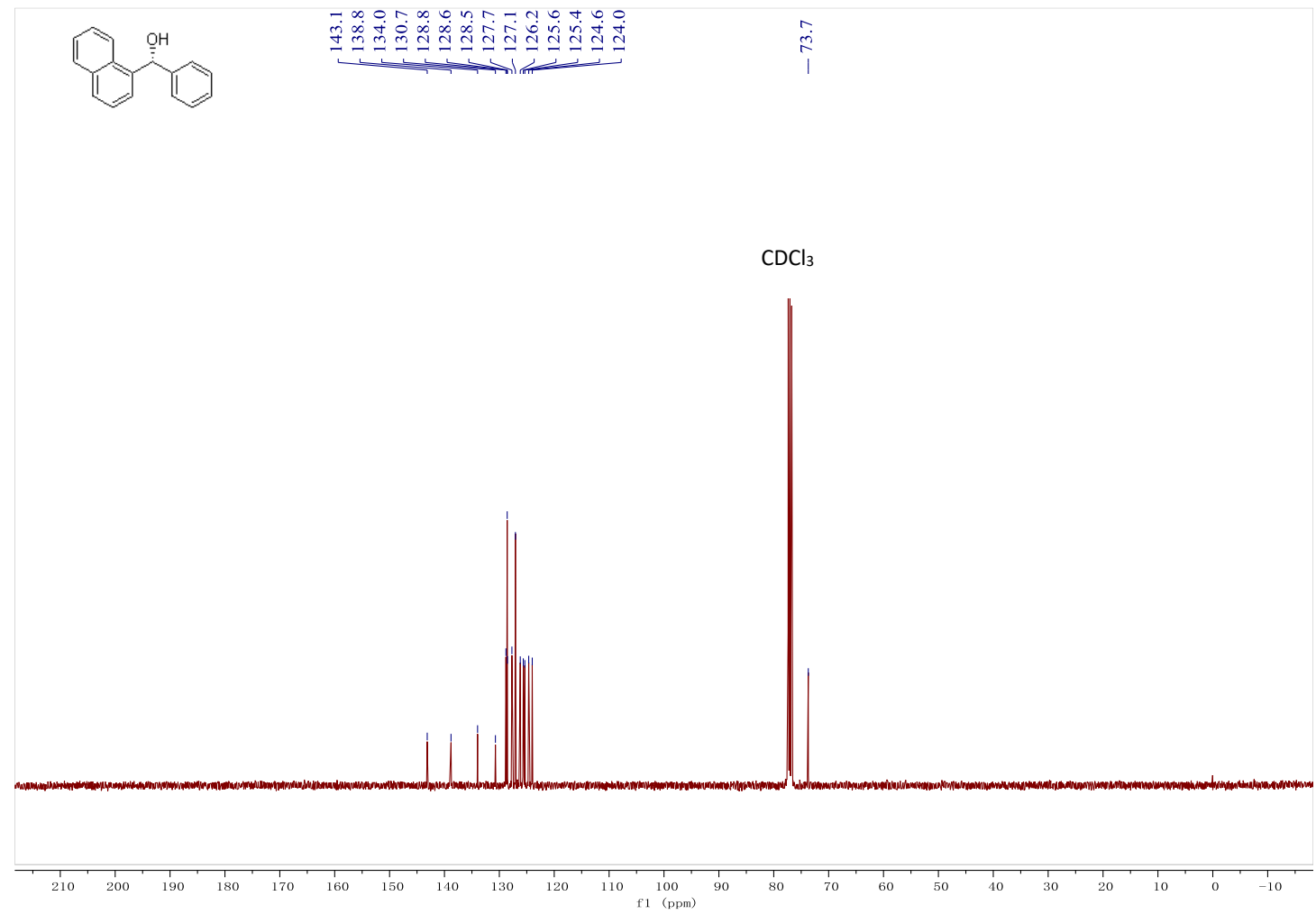
${ }^{1} \mathrm{H}$ NMR (400 MHz, $\left.\mathrm{CDCl}_{3}\right)$ of $10 \mathrm{~h}$

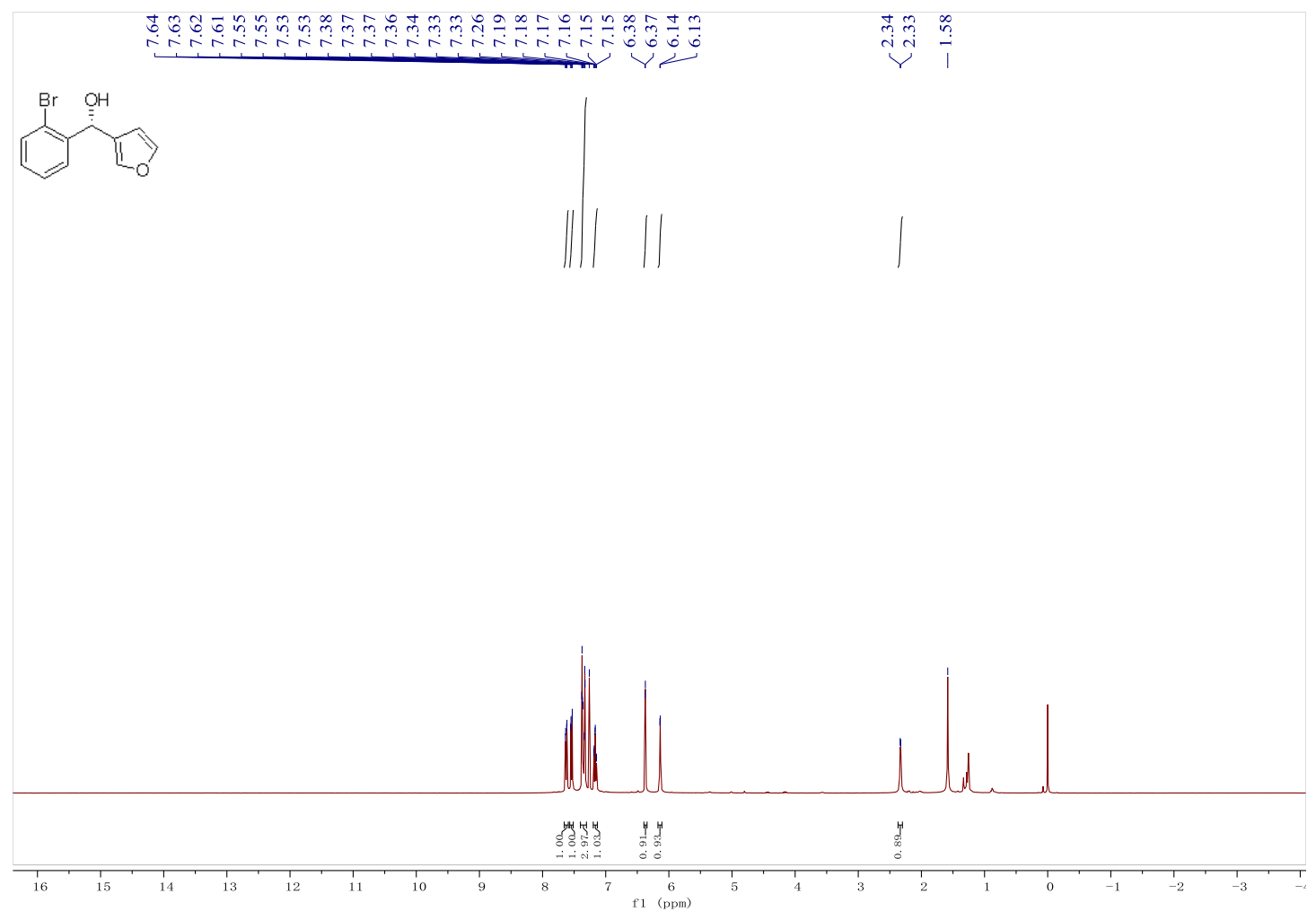

${ }^{13} \mathrm{C}$ NMR (101 MHz, $\left.\mathrm{CDCl}_{3}\right)$ of $10 \mathrm{~h}$

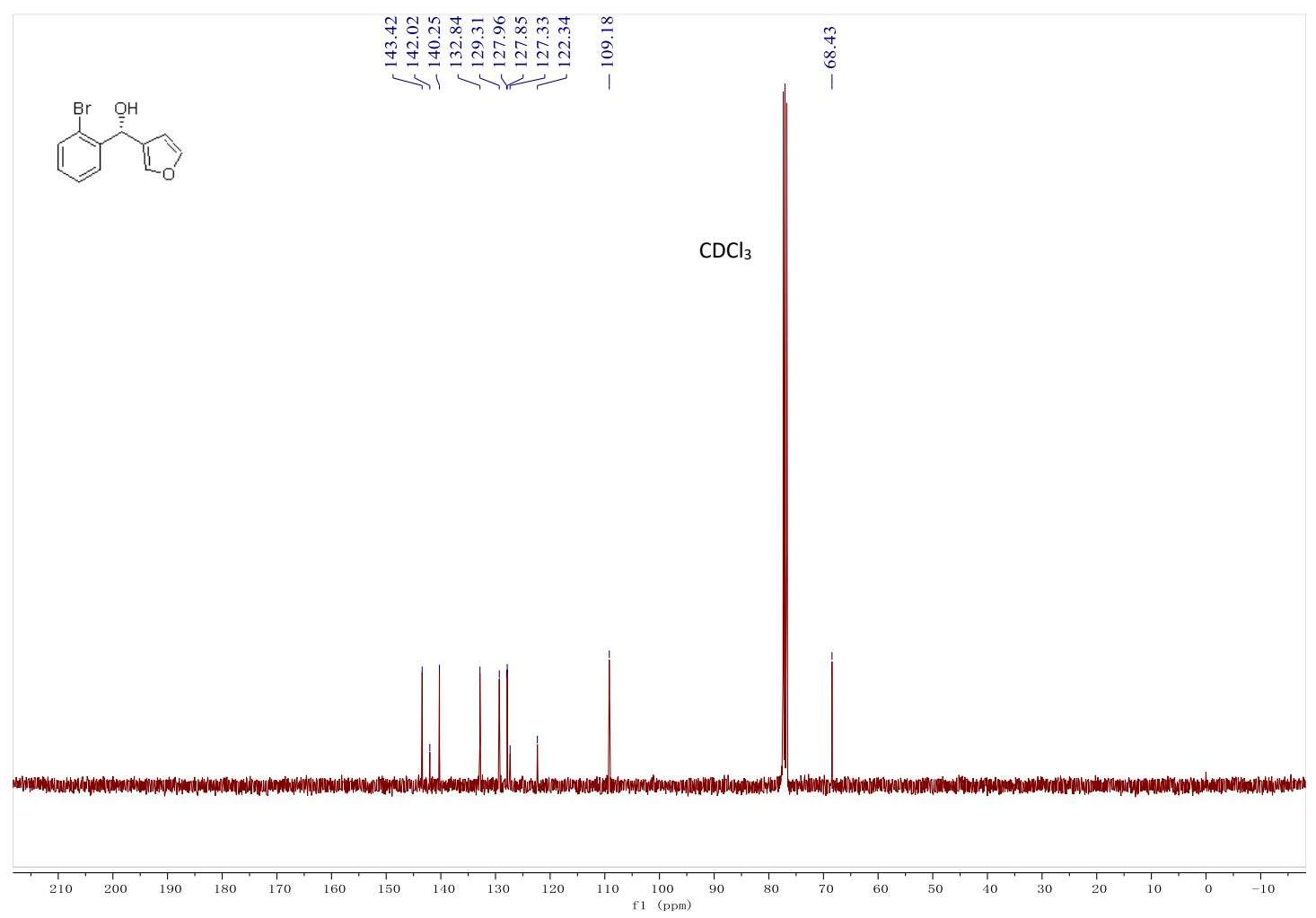

S87 
${ }^{1} \mathrm{H}$ NMR (400 MHz, $\left.\mathrm{CDCl}_{3}\right)$ of $10 \mathrm{i}$

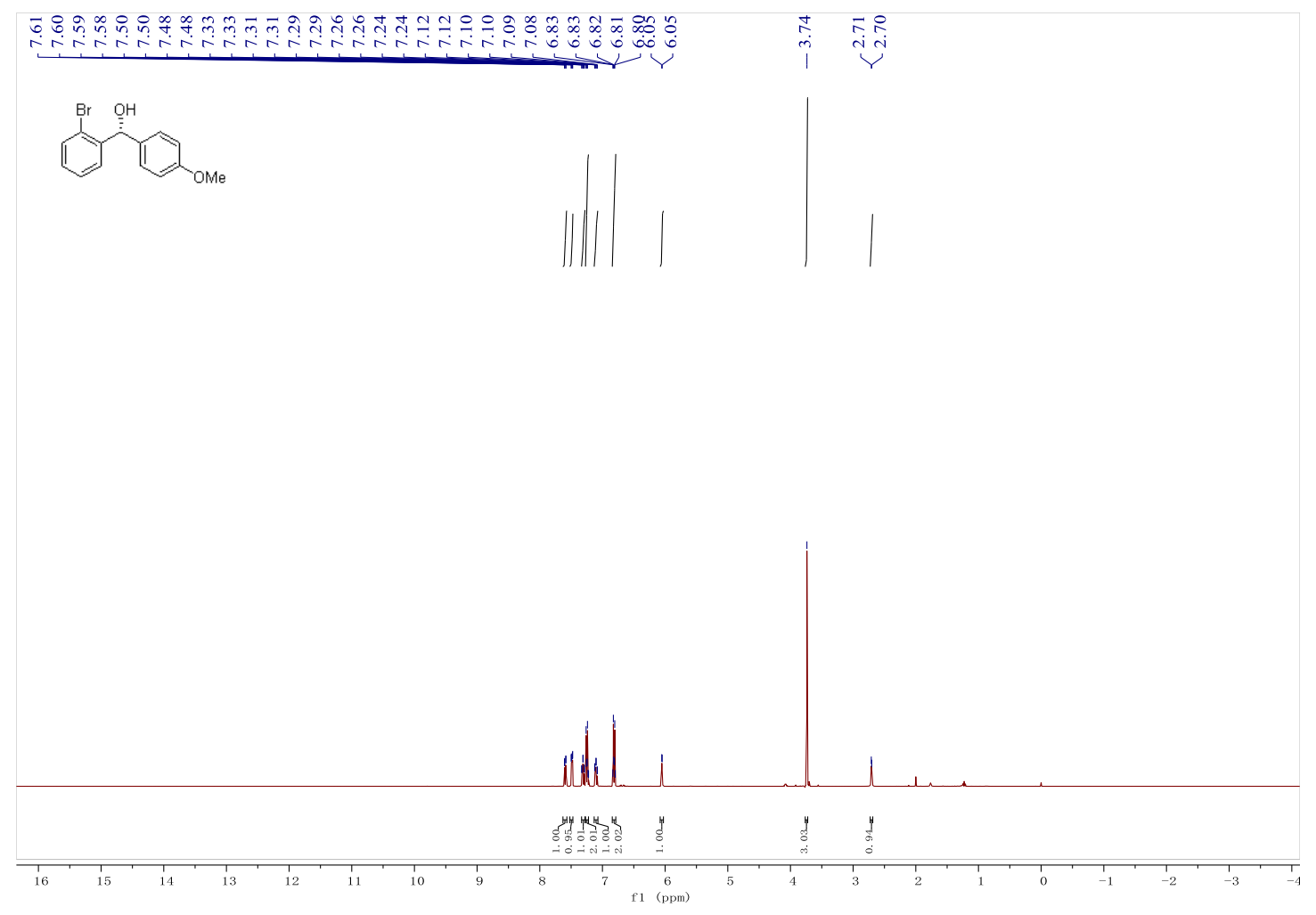

${ }^{13} \mathrm{C}$ NMR (101 MHz, $\left.\mathrm{CDCl}_{3}\right)$ of $10 \mathrm{i}$

11
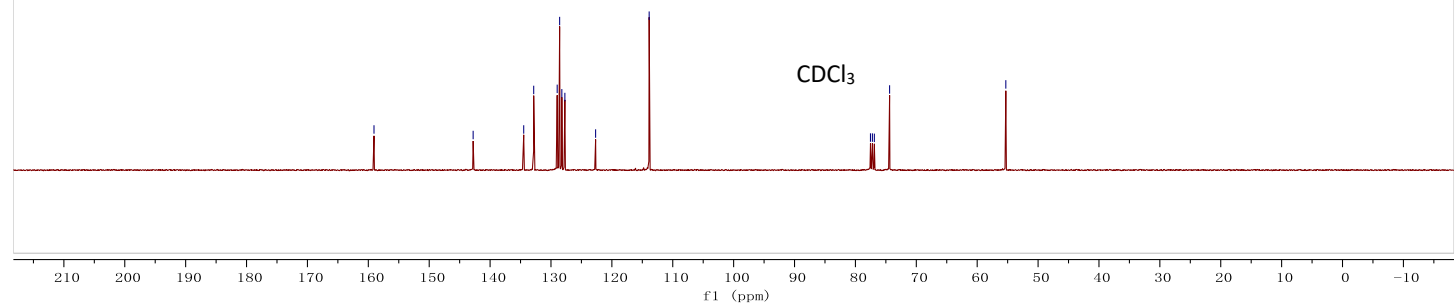
${ }^{1} \mathrm{H}$ NMR (600 MHz, $\left.\mathrm{CDCl}_{3}\right)$ of $10 \mathrm{j}$

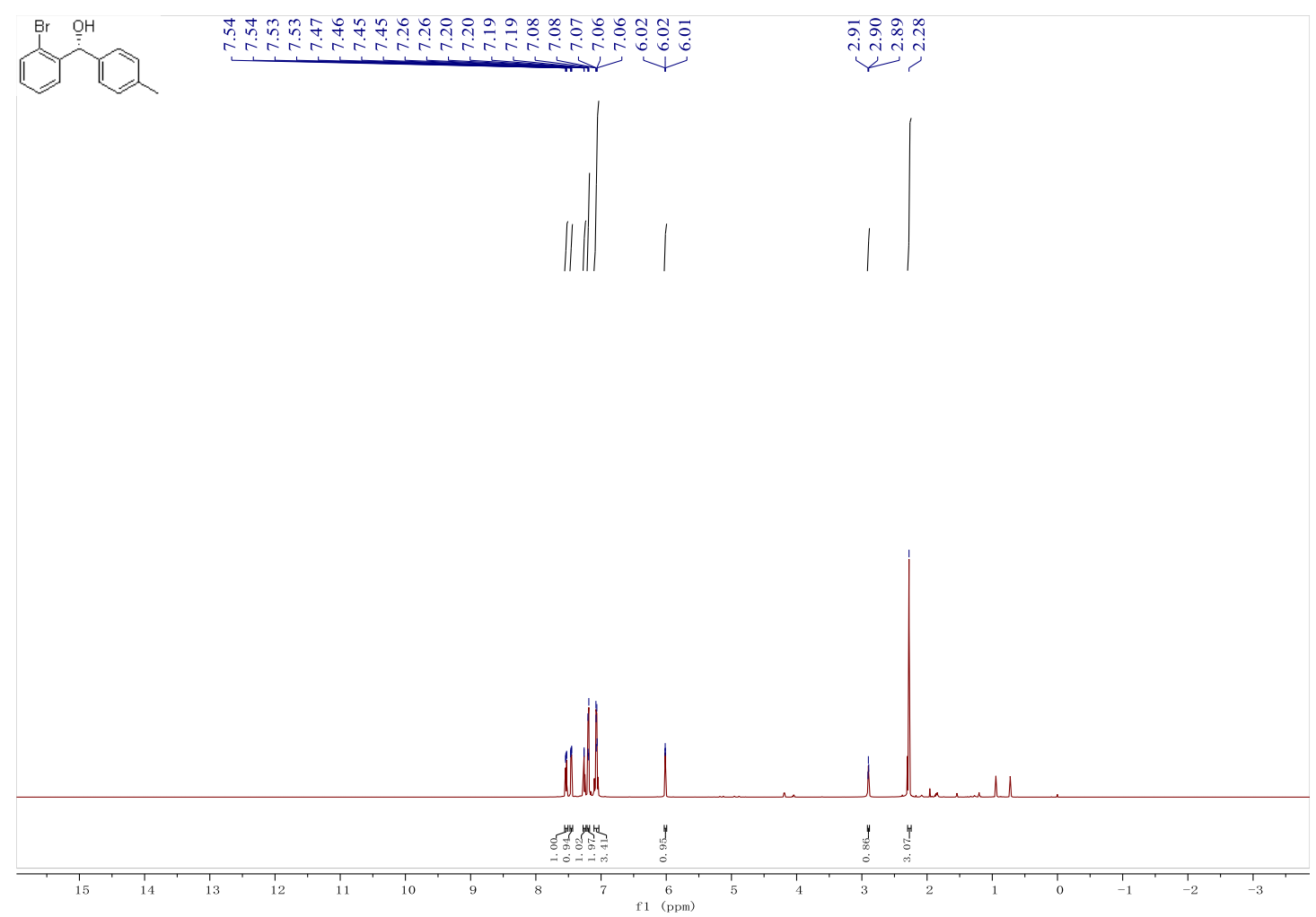

${ }^{13} \mathrm{C}$ NMR (151 MHz, CDCl $)$ of 10j

11
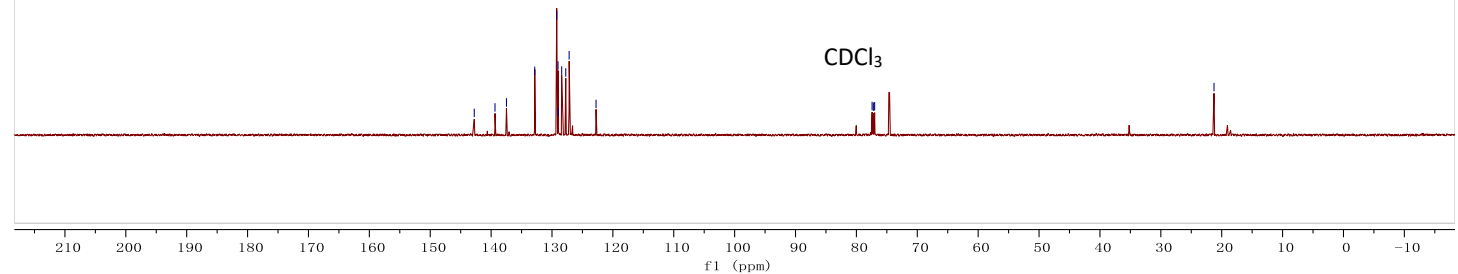
${ }^{1} \mathrm{H}$ NMR (600 MHz, $\left.\mathrm{CDCl}_{3}\right)$ of $10 \mathrm{k}$

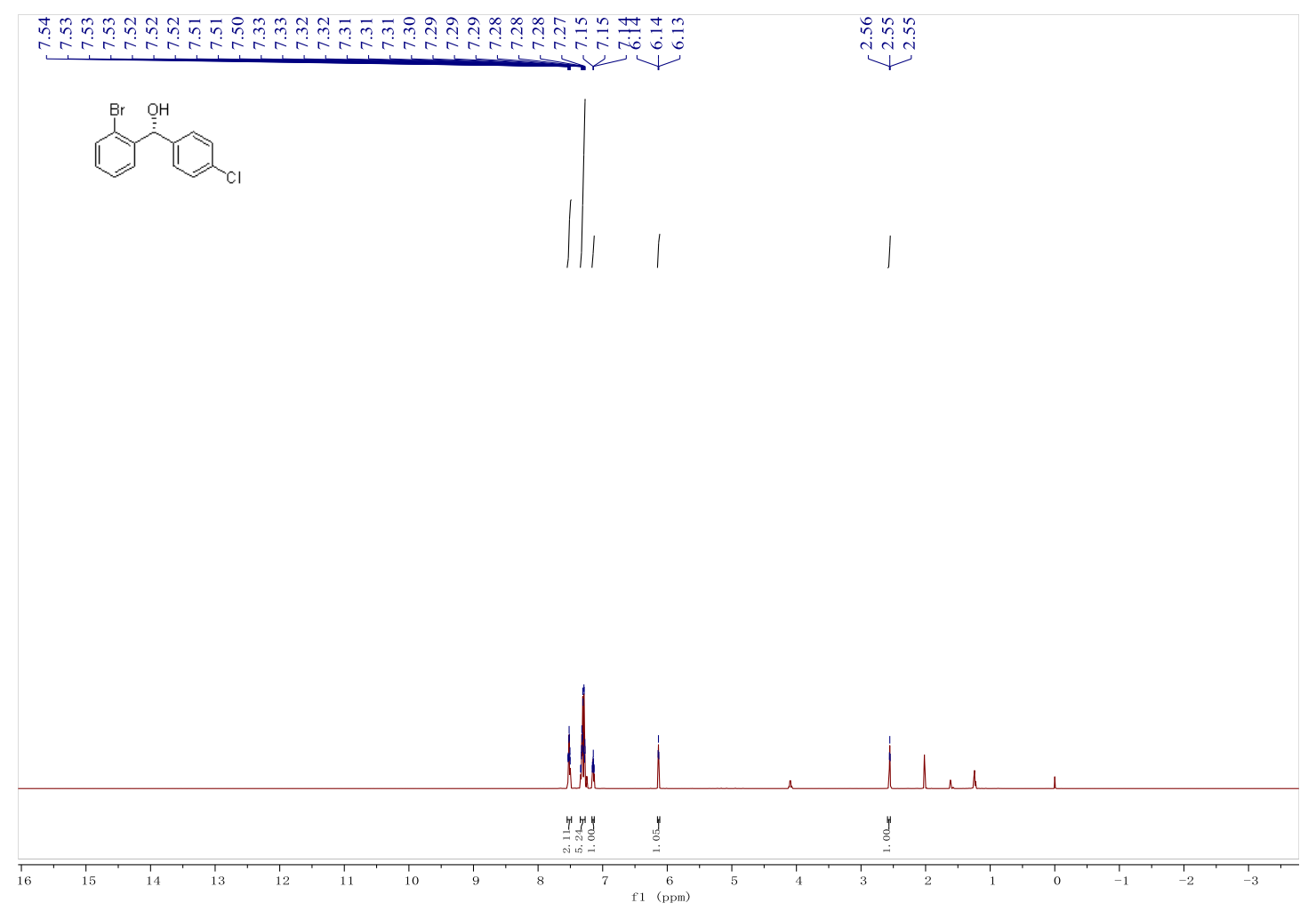

${ }^{13} \mathrm{C}$ NMR (151 MHz, CDCl 3$)$ of 10k
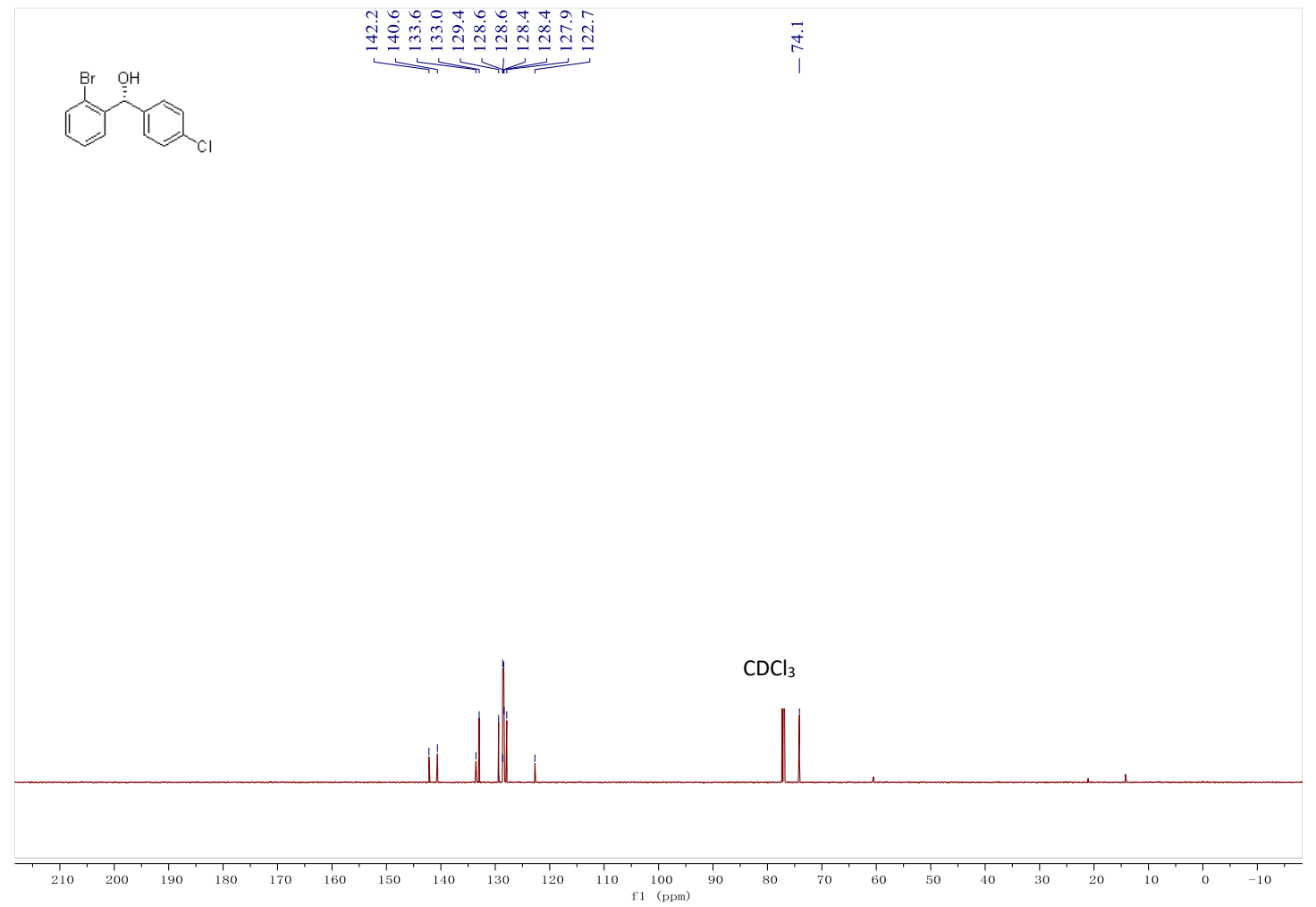
${ }^{1} \mathrm{H}$ NMR (600 MHz, $\left.\mathrm{CDCl}_{3}\right)$ of 101

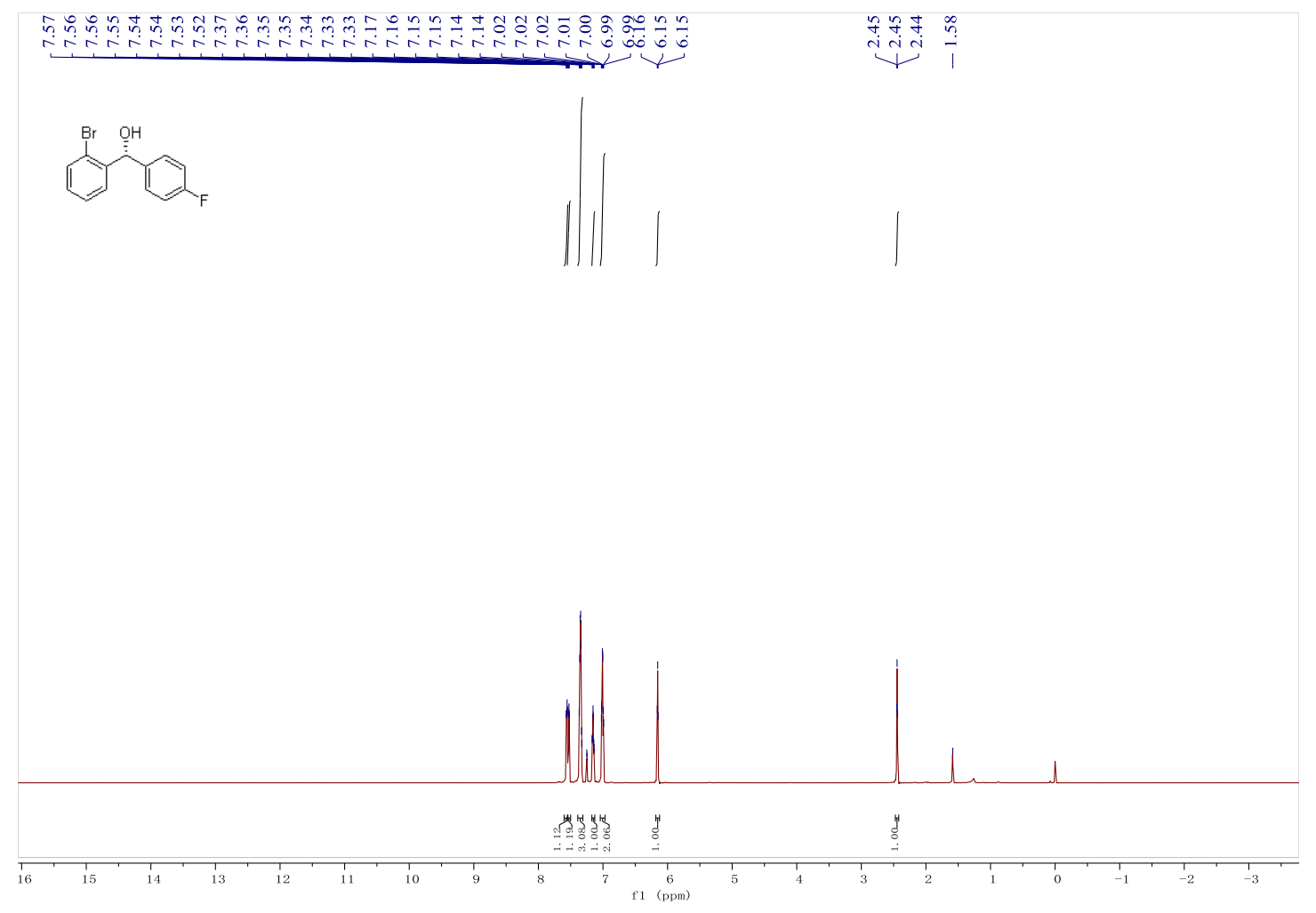

${ }^{13} \mathrm{C}$ NMR (101 MHz, CDCl3) of 101

F
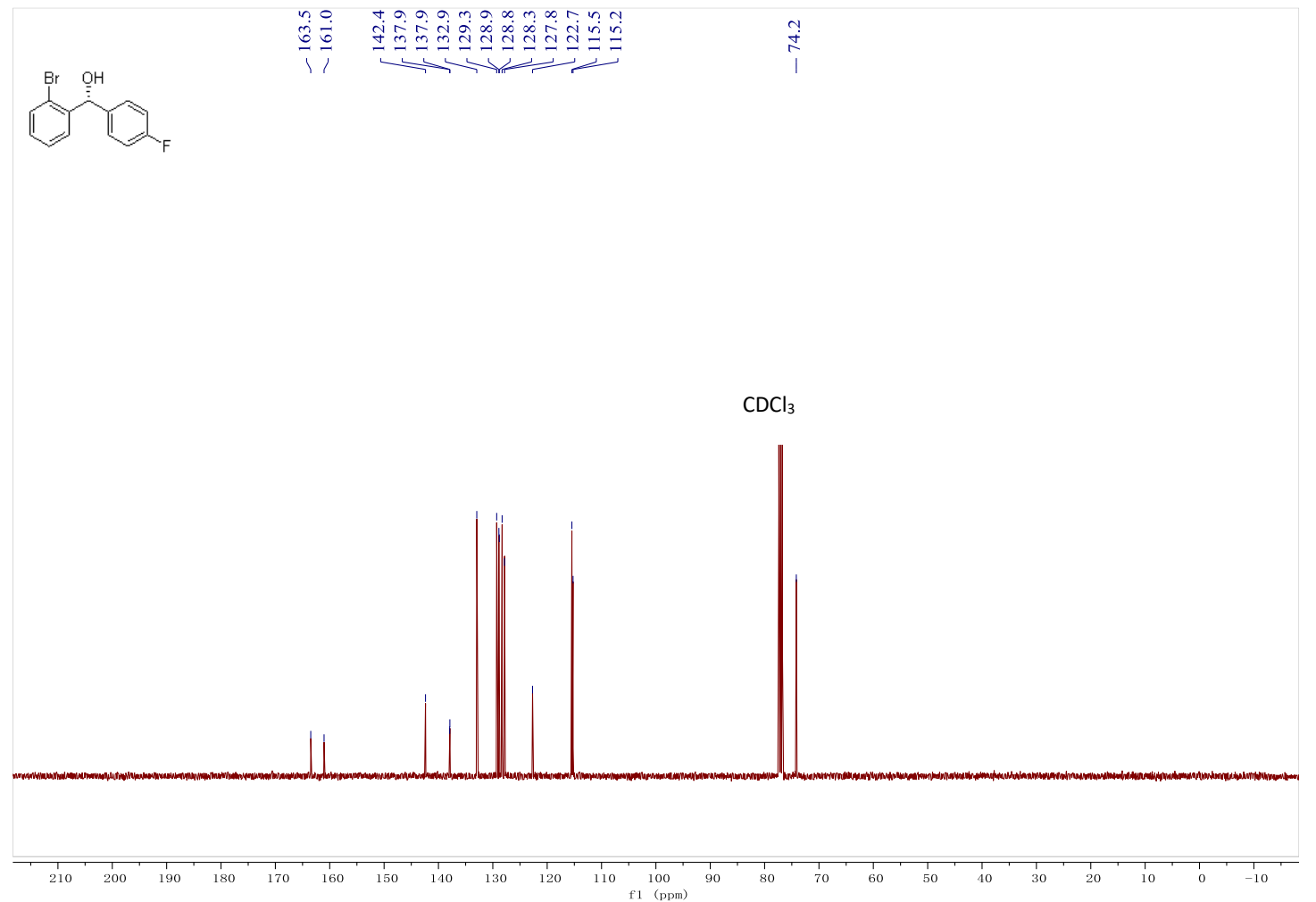
${ }^{19} \mathrm{~F}$ NMR (376 MHz, $\mathrm{CDCl}_{3}$ ) of $10 \mathrm{I}$
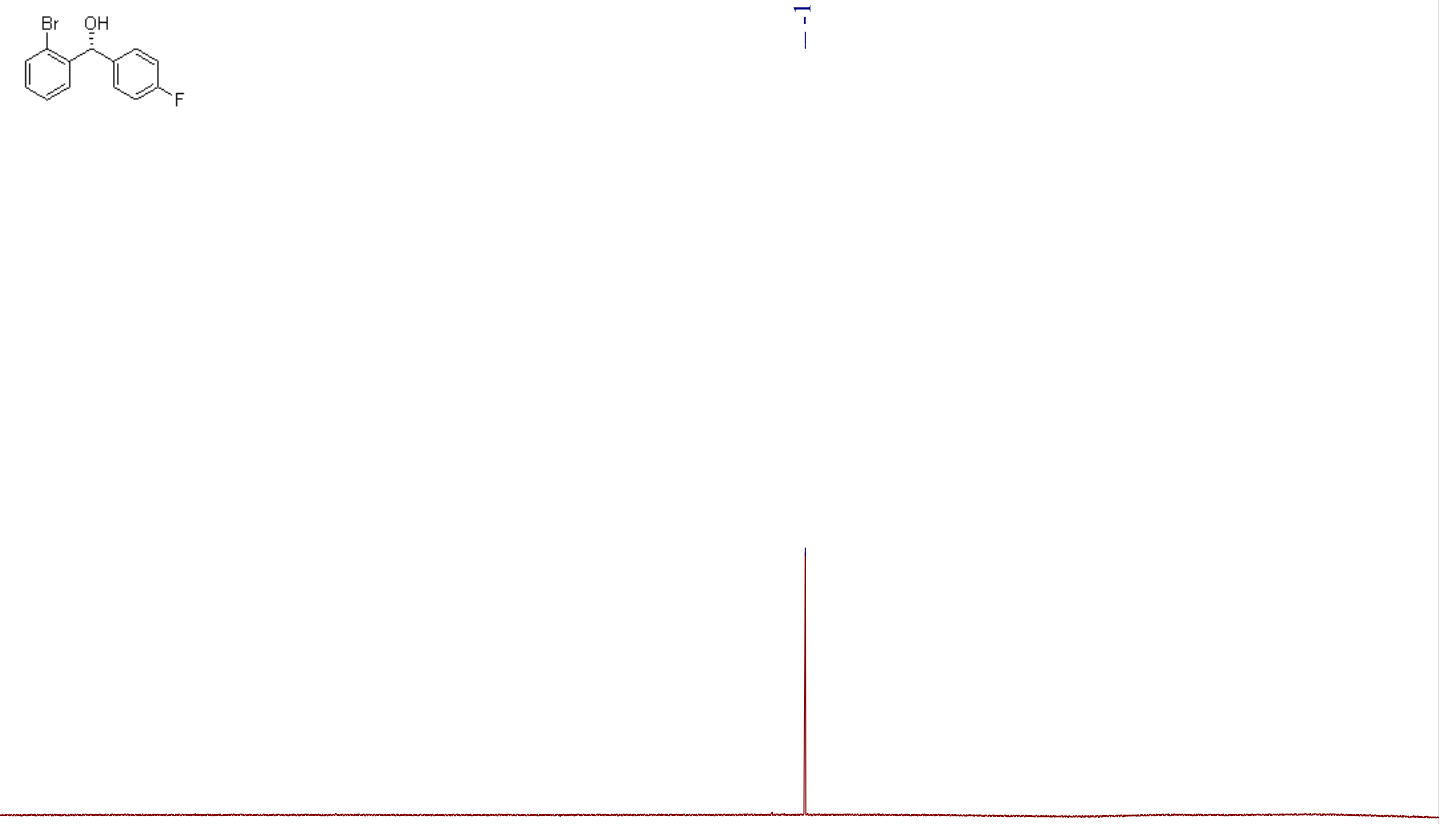

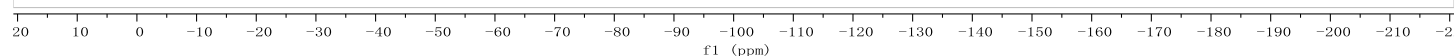


${ }^{1} \mathrm{H}$ NMR (400 MHz, CDCl $)$ of $10 \mathrm{~m}$

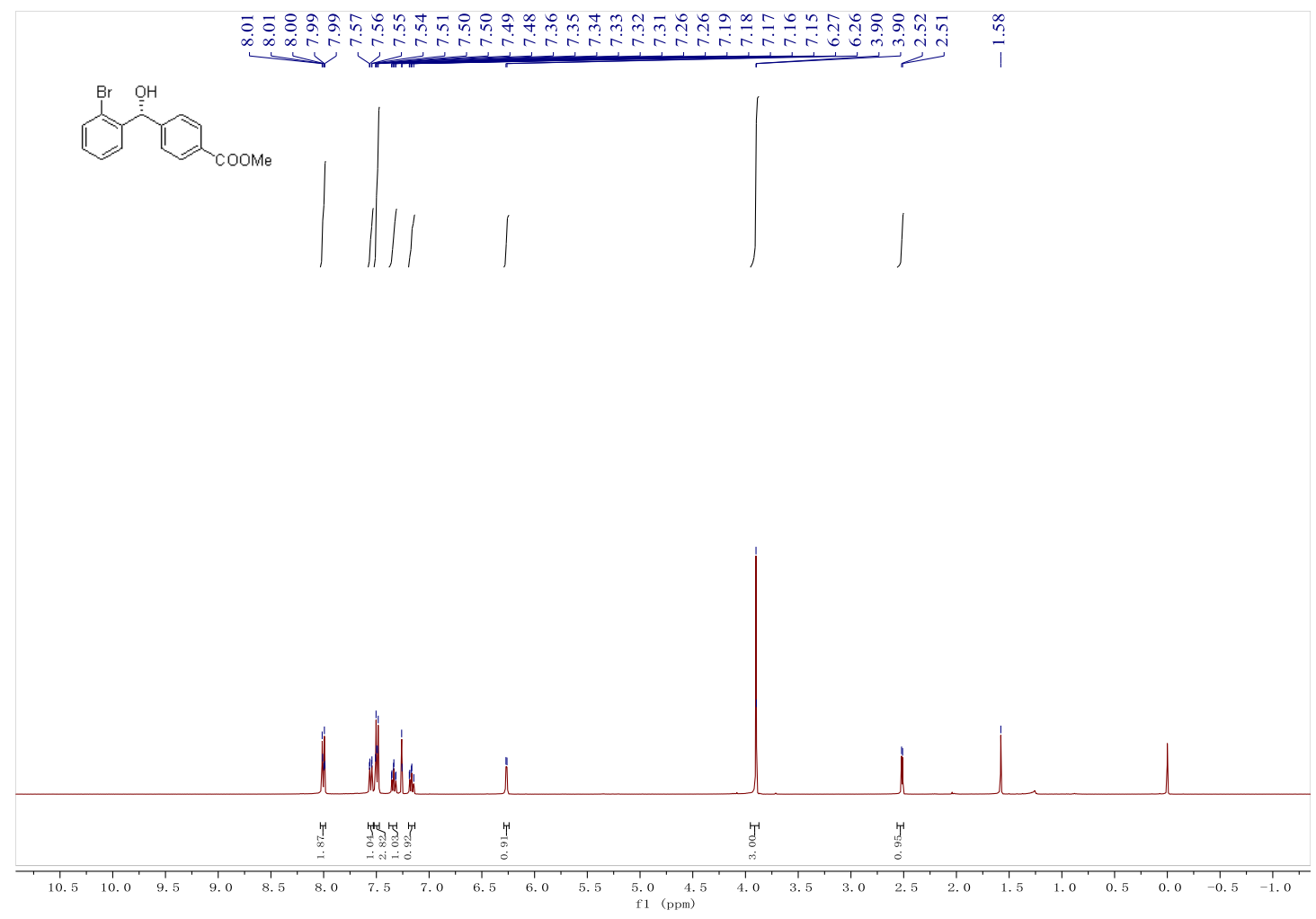

${ }^{13} \mathrm{C}$ NMR (101 MHz, $\left.\mathrm{CDCl}_{3}\right)$ of $10 \mathrm{~m}$

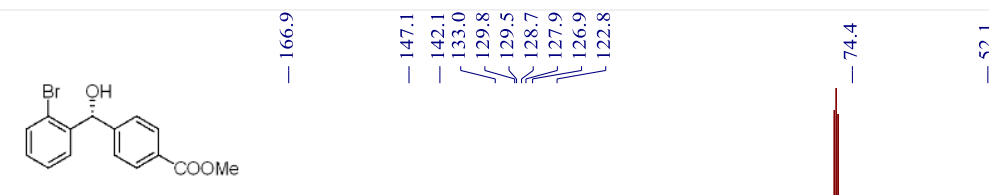

$\mathrm{CDCl}_{3}$
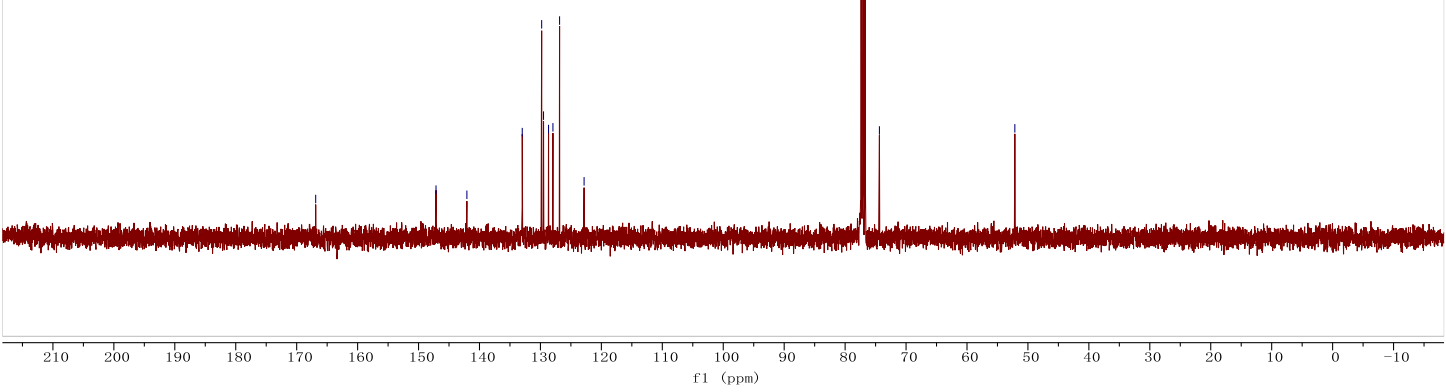
${ }^{1} \mathrm{H}$ NMR (400 MHz, $\left.\mathrm{CDCl}_{3}\right)$ of $10 \mathrm{n}$

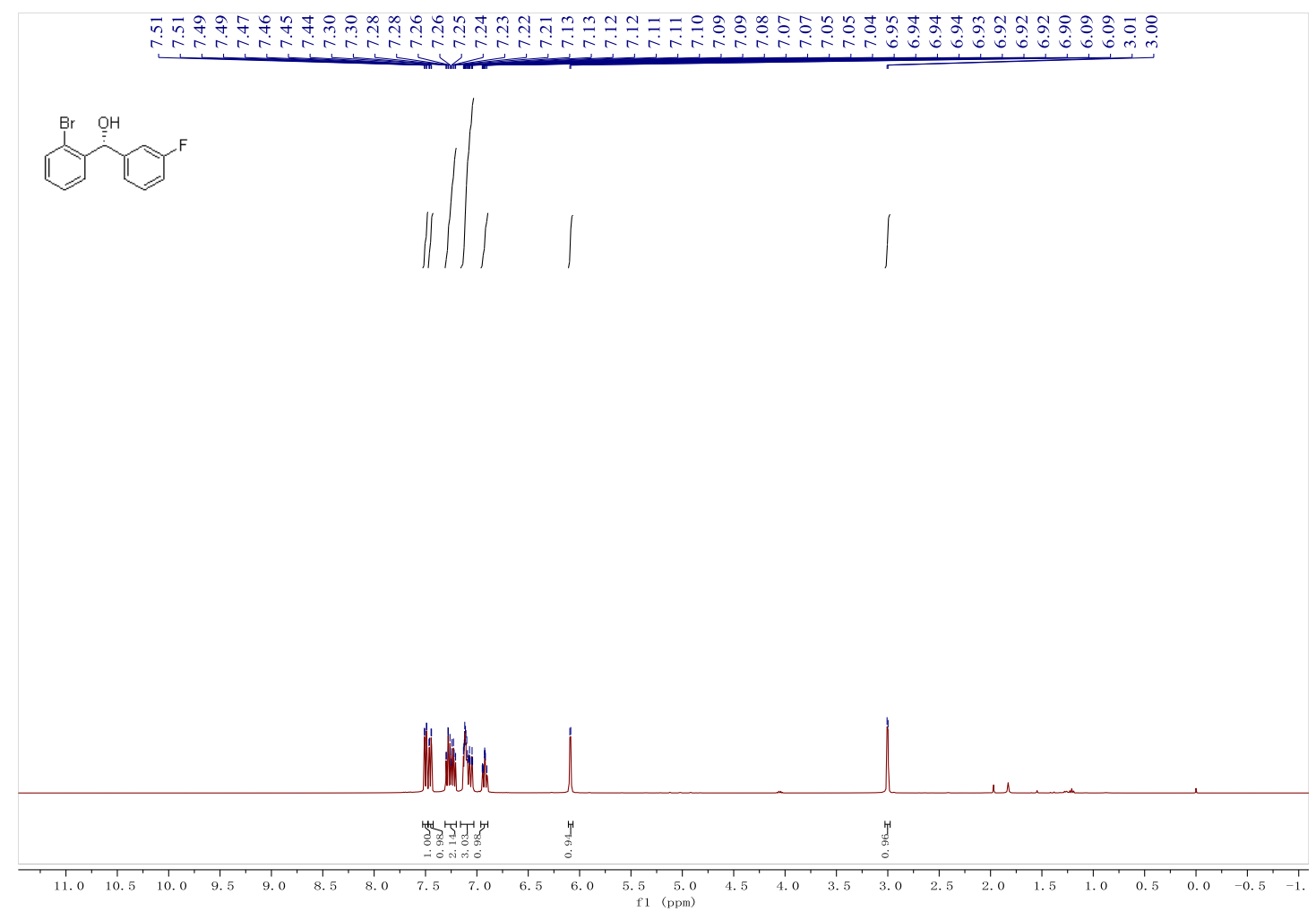

${ }^{13} \mathrm{C}$ NMR (101 MHz, CDCl 3$)$ of 10n
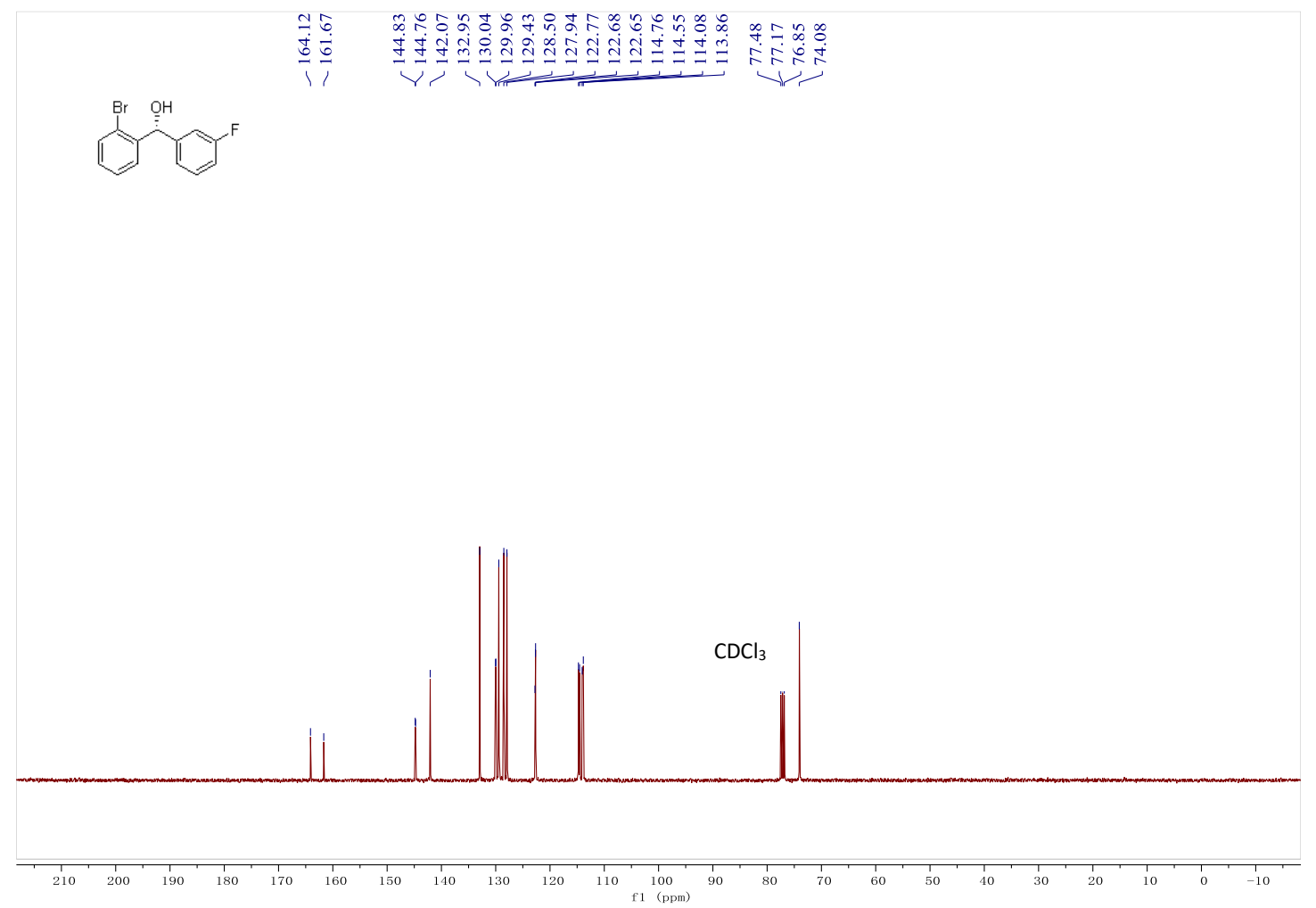
${ }^{19}$ F NMR (376 MHz, $\left.\mathrm{CDCl}_{3}\right)$ of $10 \mathrm{n}$

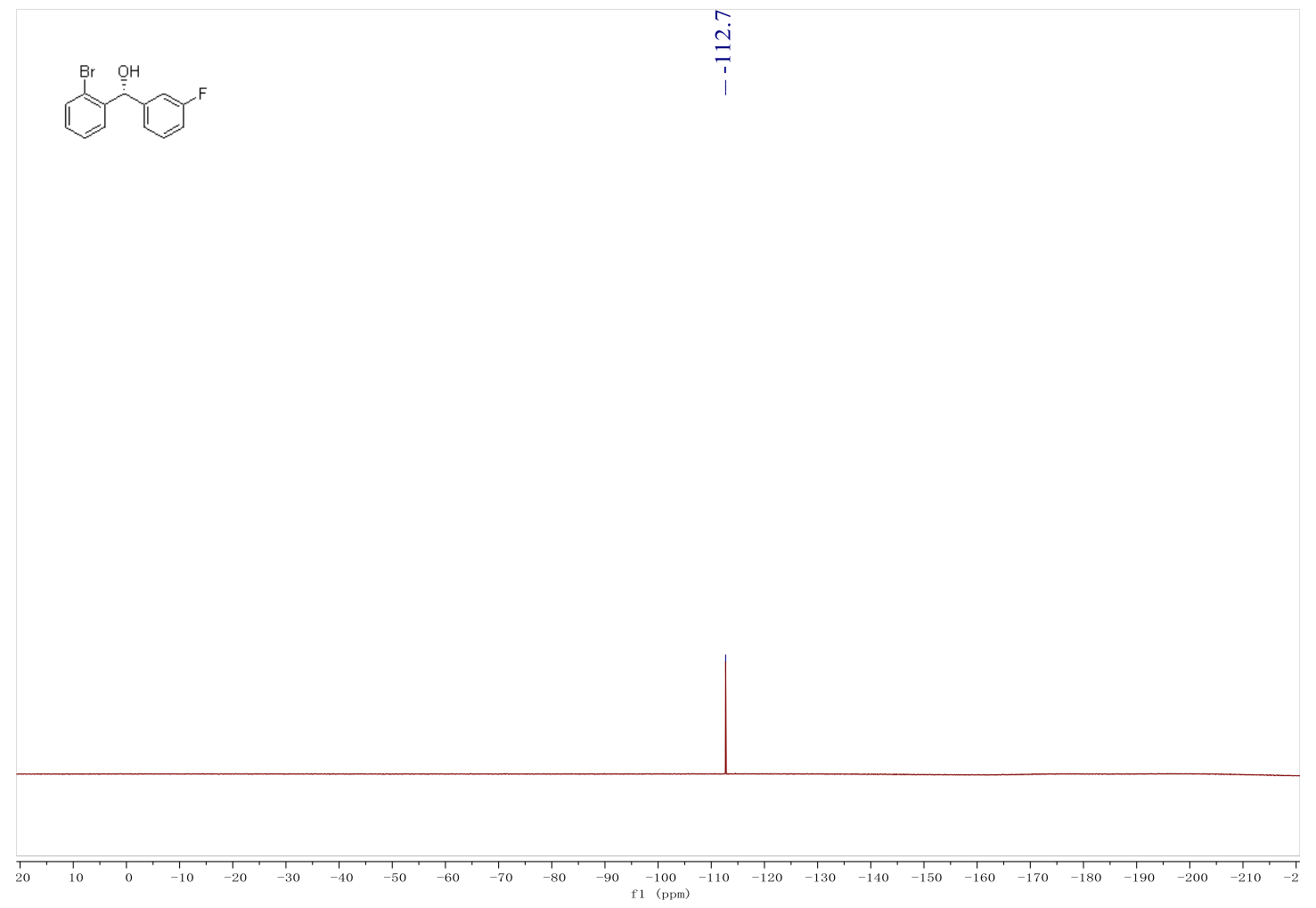


${ }^{1} \mathrm{H}$ NMR (400 $\left.\mathrm{MHz}, \mathrm{CDCl}_{3}\right)$ of 100

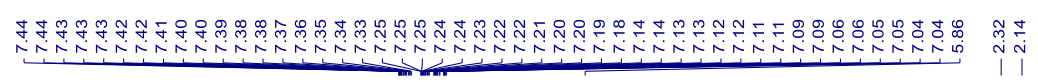

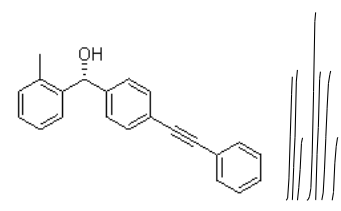

1

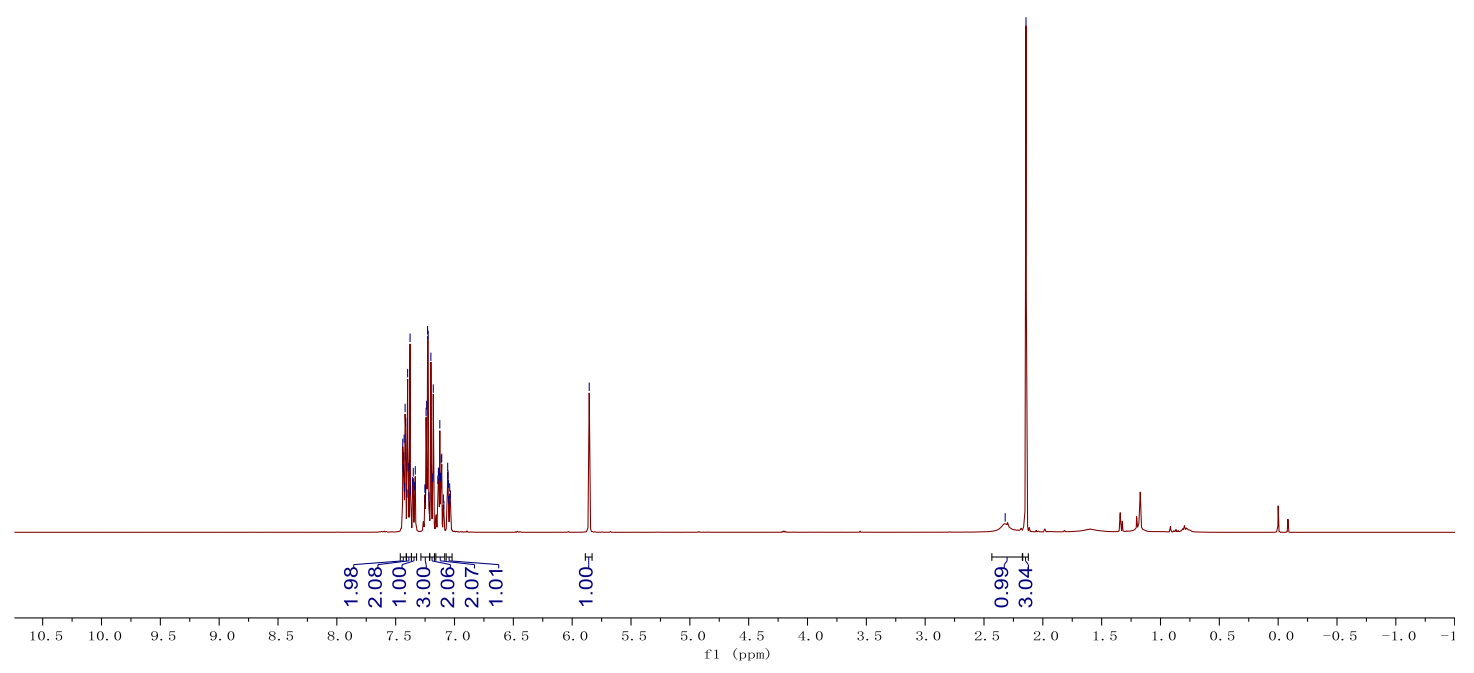

${ }^{13} \mathrm{C}$ NMR (101 MHz, $\left.\mathrm{CDCl}_{3}\right)$ of 100
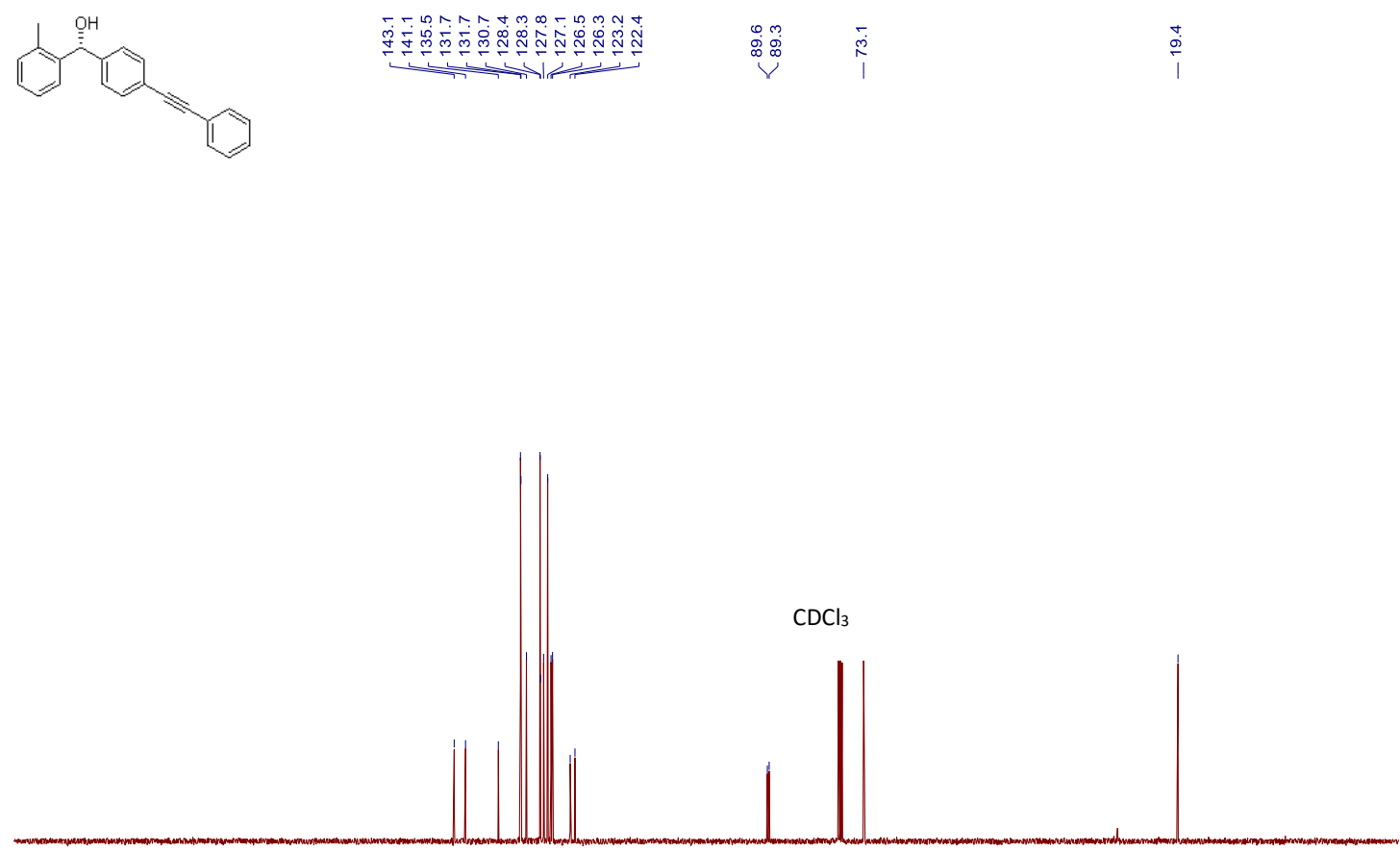

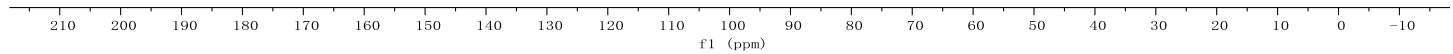


${ }^{1} \mathrm{H}$ NMR (400 MHz, $\left.\mathrm{CDCl}_{3}\right)$ of 10p
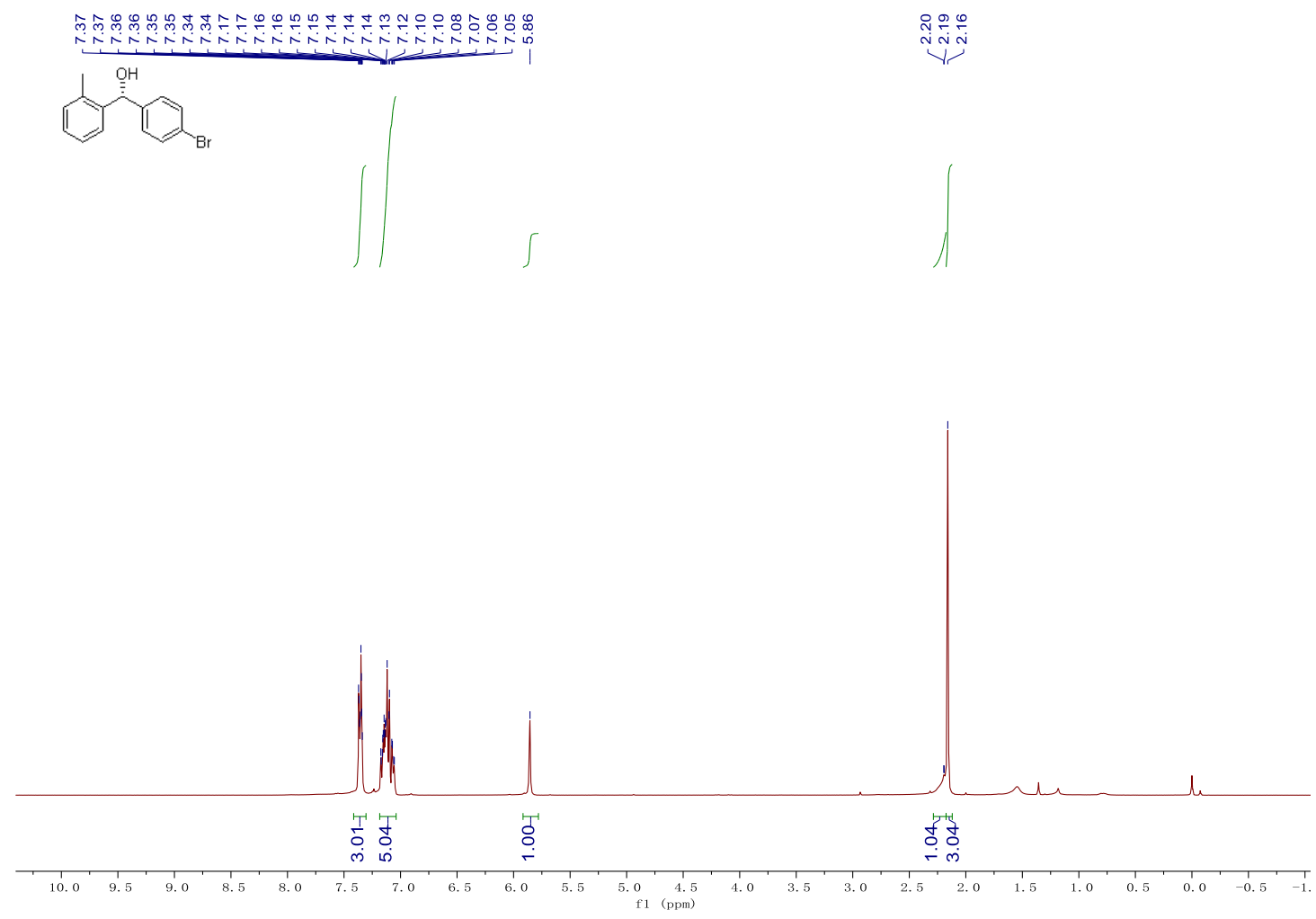

${ }^{13} \mathrm{C}$ NMR (101 MHz, $\left.\mathrm{CDCl}_{3}\right)$ of 10p
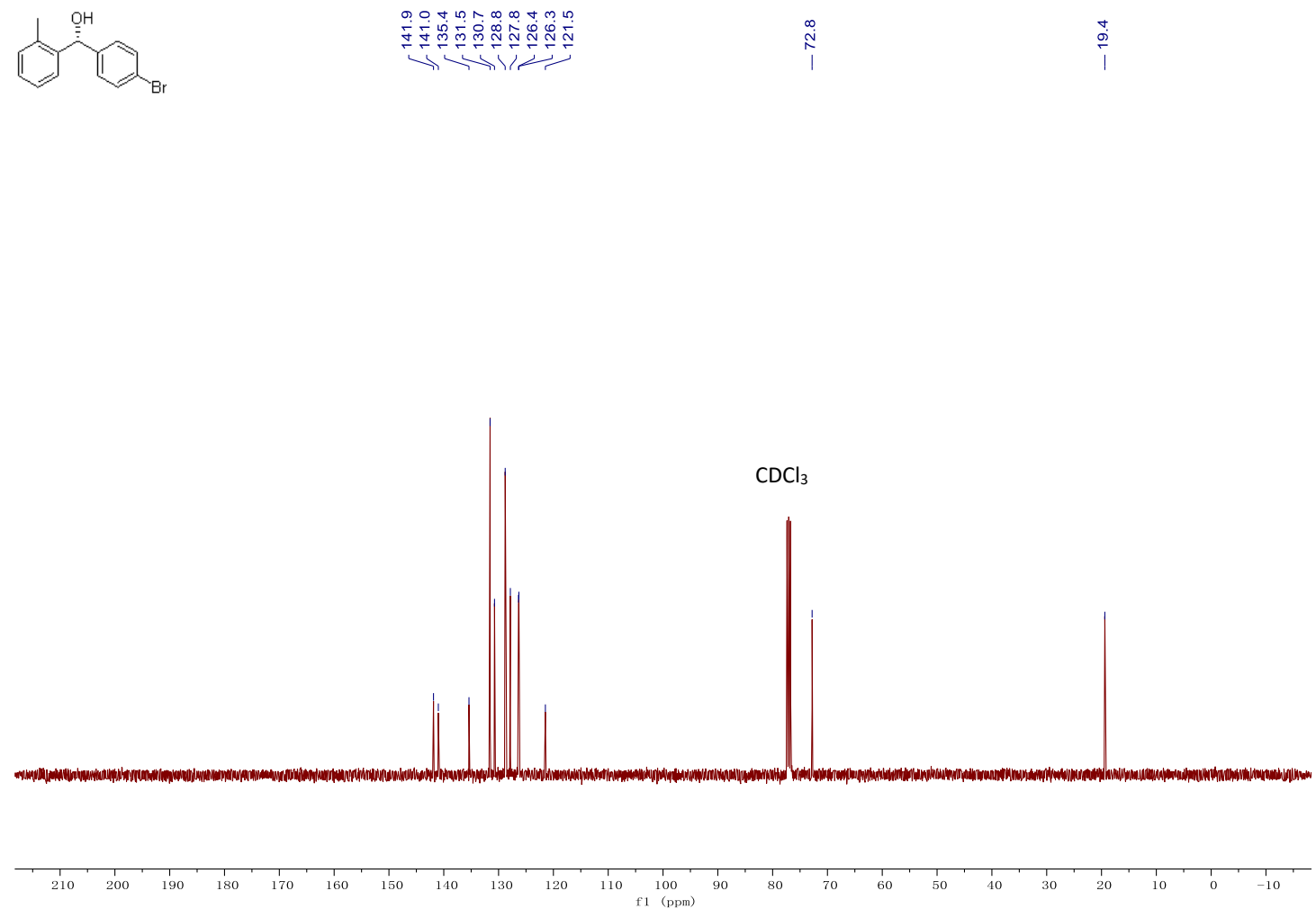
${ }^{1} \mathrm{H}$ NMR (400 MHz, $\left.\mathrm{CDCl}_{3}\right)$ of $10 \mathrm{q}$
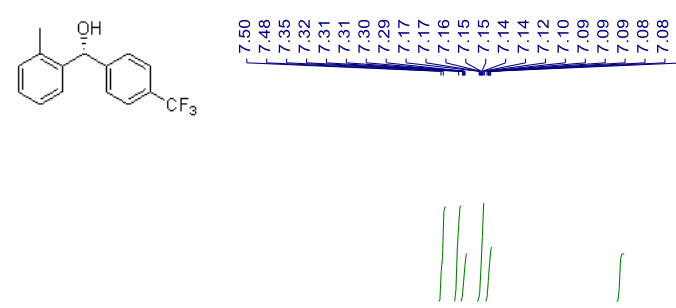

ฟูำ

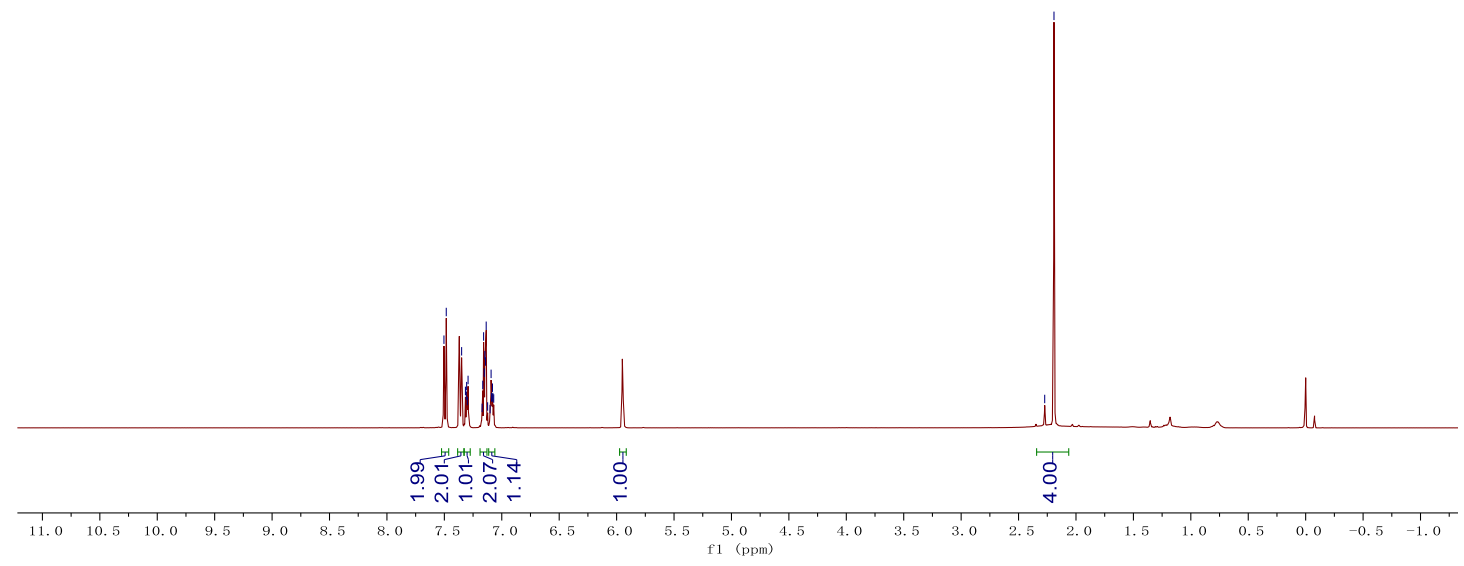

${ }^{13} \mathrm{C}$ NMR (101 MHz, $\left.\mathrm{CDCl}_{3}\right)$ of 10q
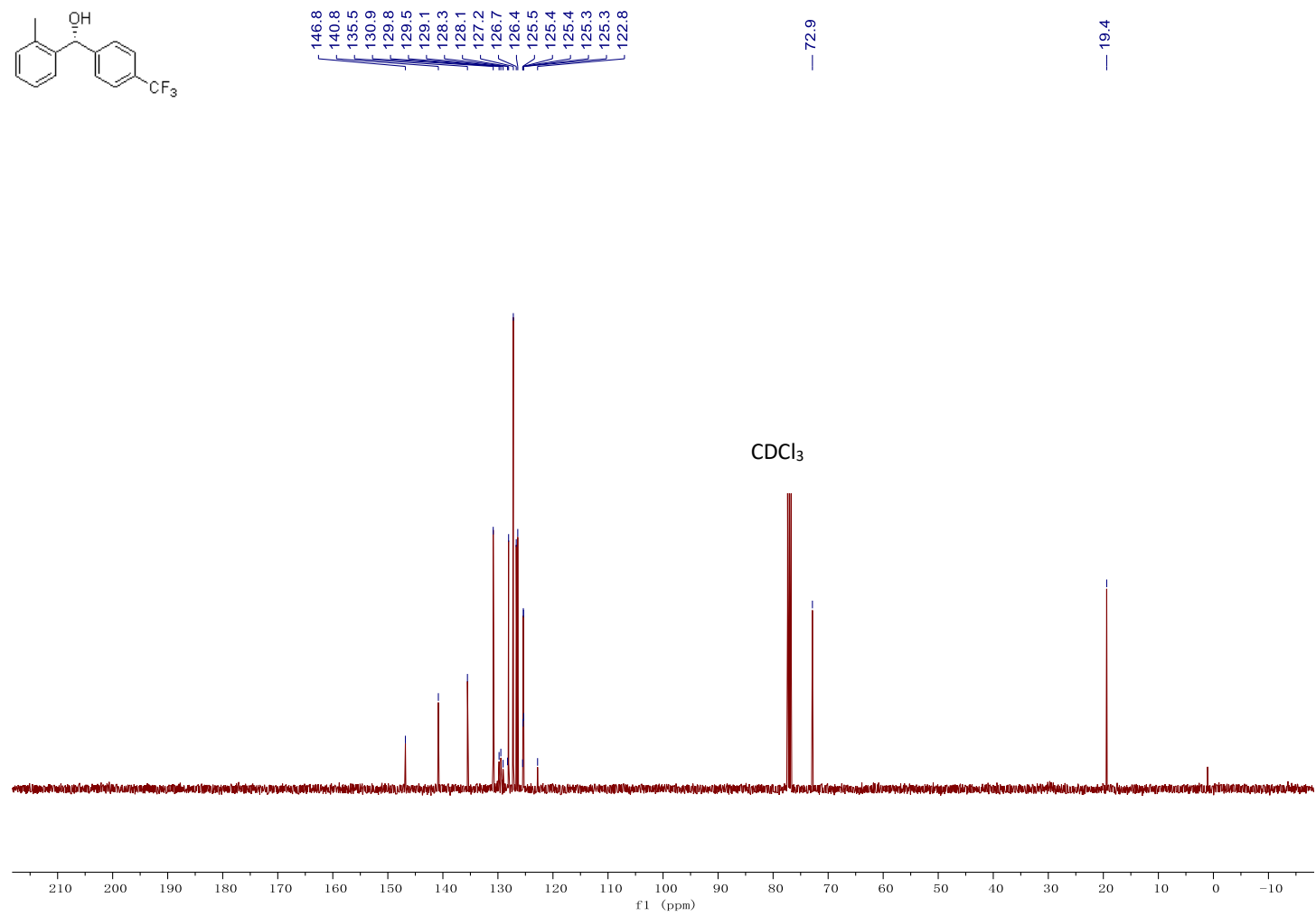
${ }^{19}$ F NMR (376 MHz, $\left.\mathrm{CDCl}_{3}\right)$ of 10q

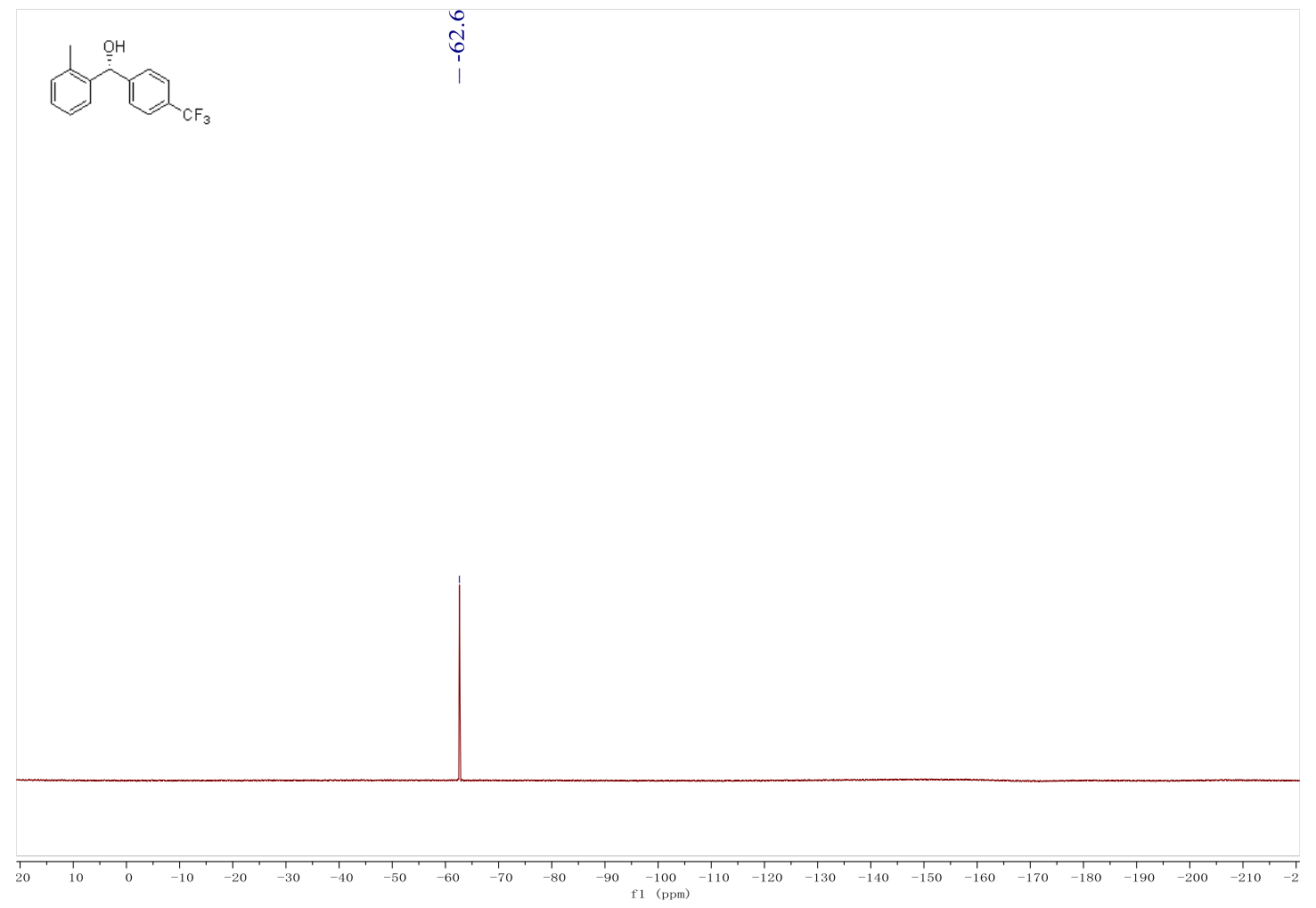


${ }^{1} \mathrm{H}$ NMR (400 MHz, $\left.\mathrm{CDCl}_{3}\right)$ of $10 \mathrm{r}$
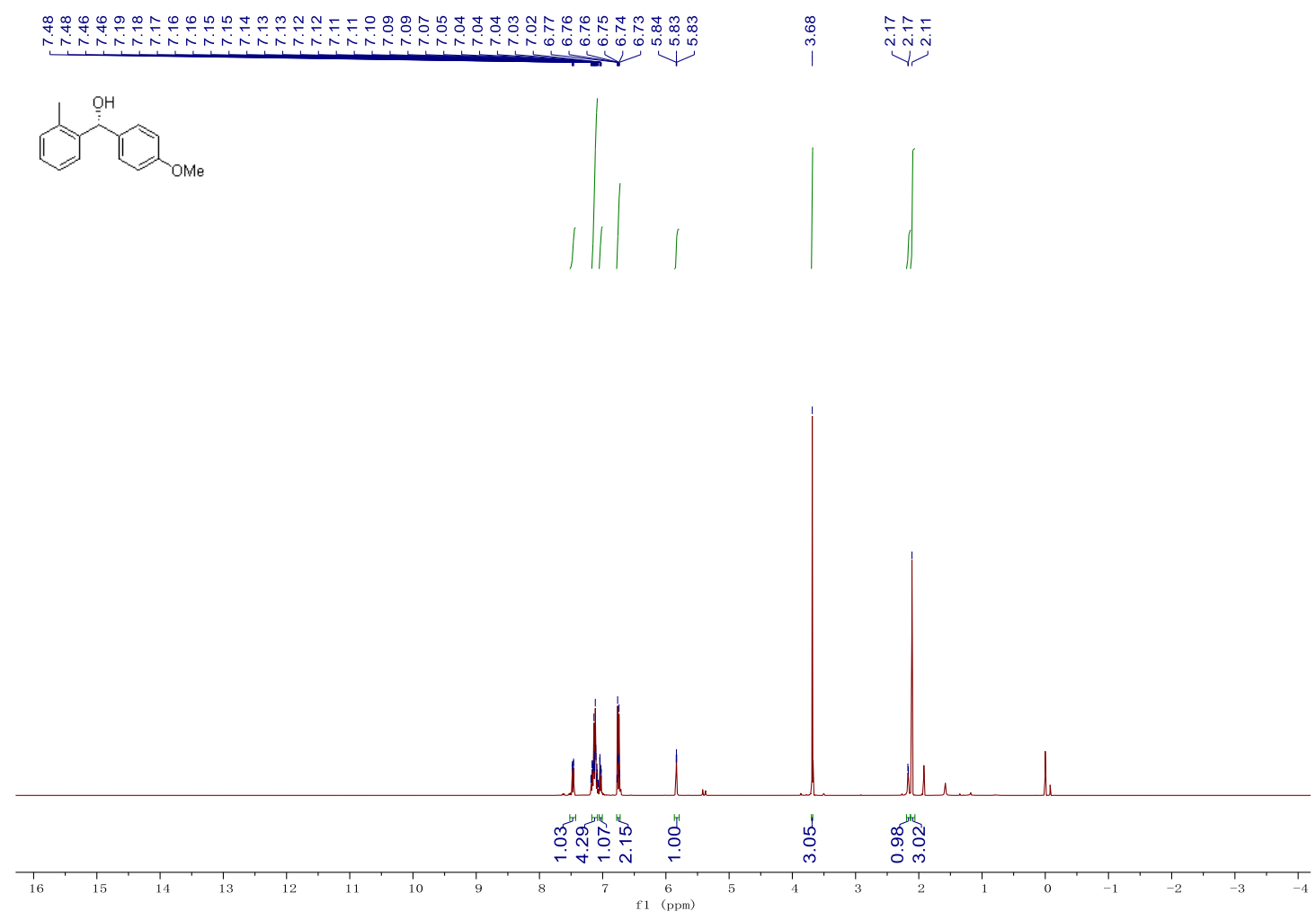

${ }^{13} \mathrm{C}$ NMR (101 MHz, $\left.\mathrm{CDCl}_{3}\right)$ of $10 \mathrm{r}$

il
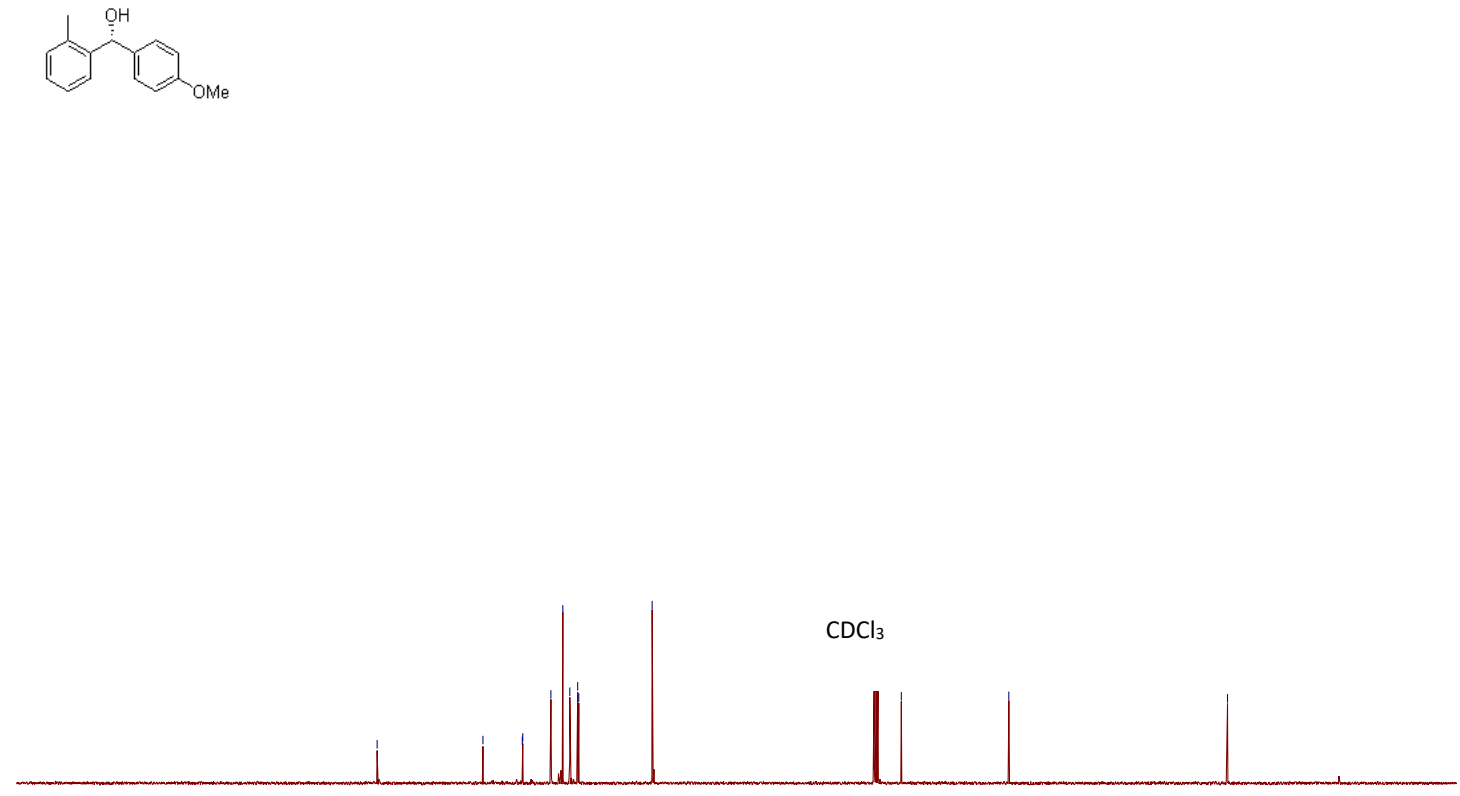

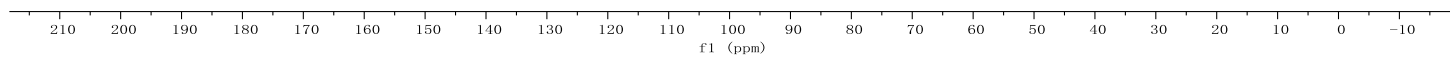


${ }^{1} \mathrm{H}$ NMR (400 MHz, $\mathrm{CDCl}_{3}$ ) of $10 \mathrm{~s}$
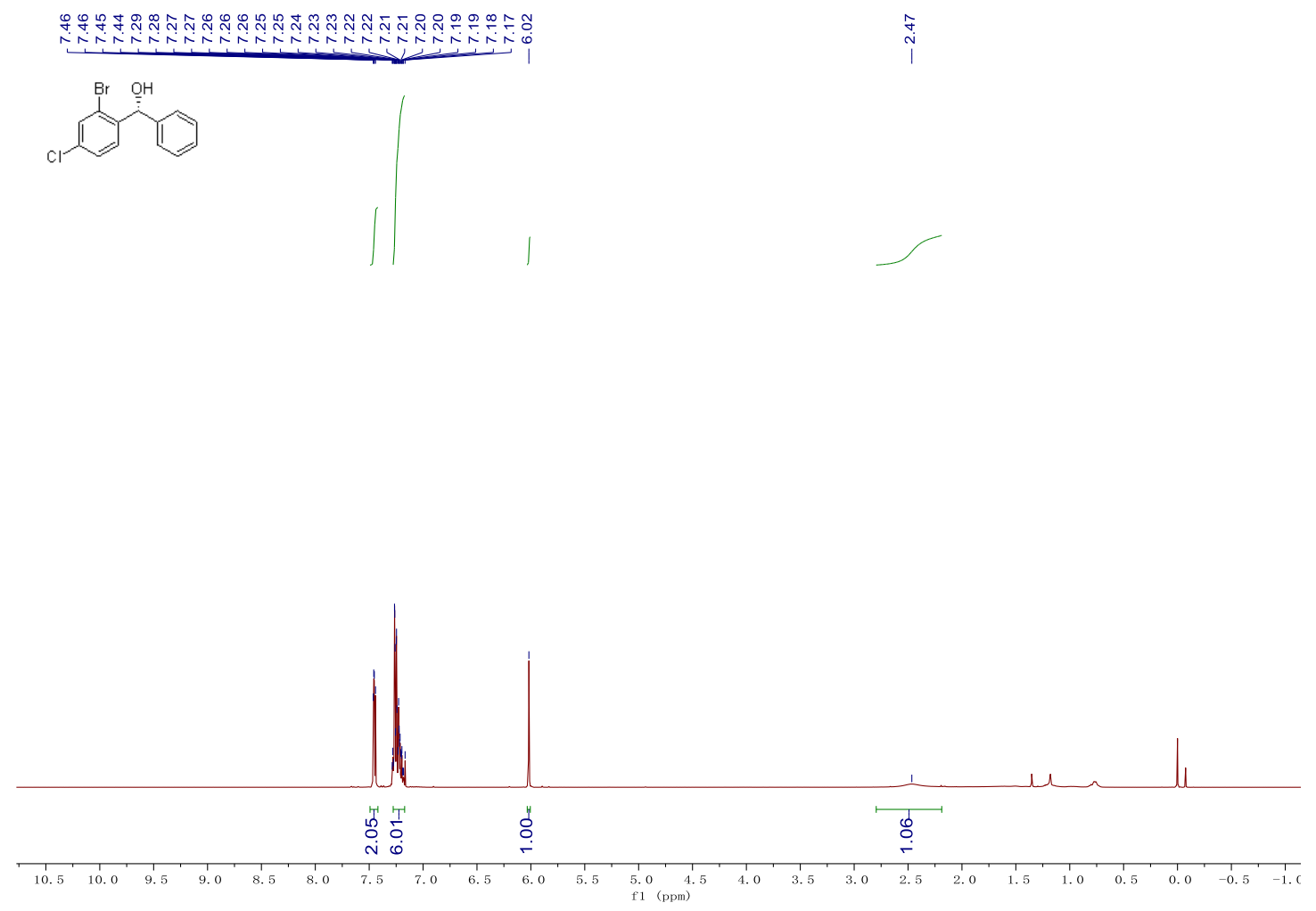

${ }^{13} \mathrm{C}$ NMR (101 MHz, $\left.\mathrm{CDCl}_{3}\right)$ of 10s
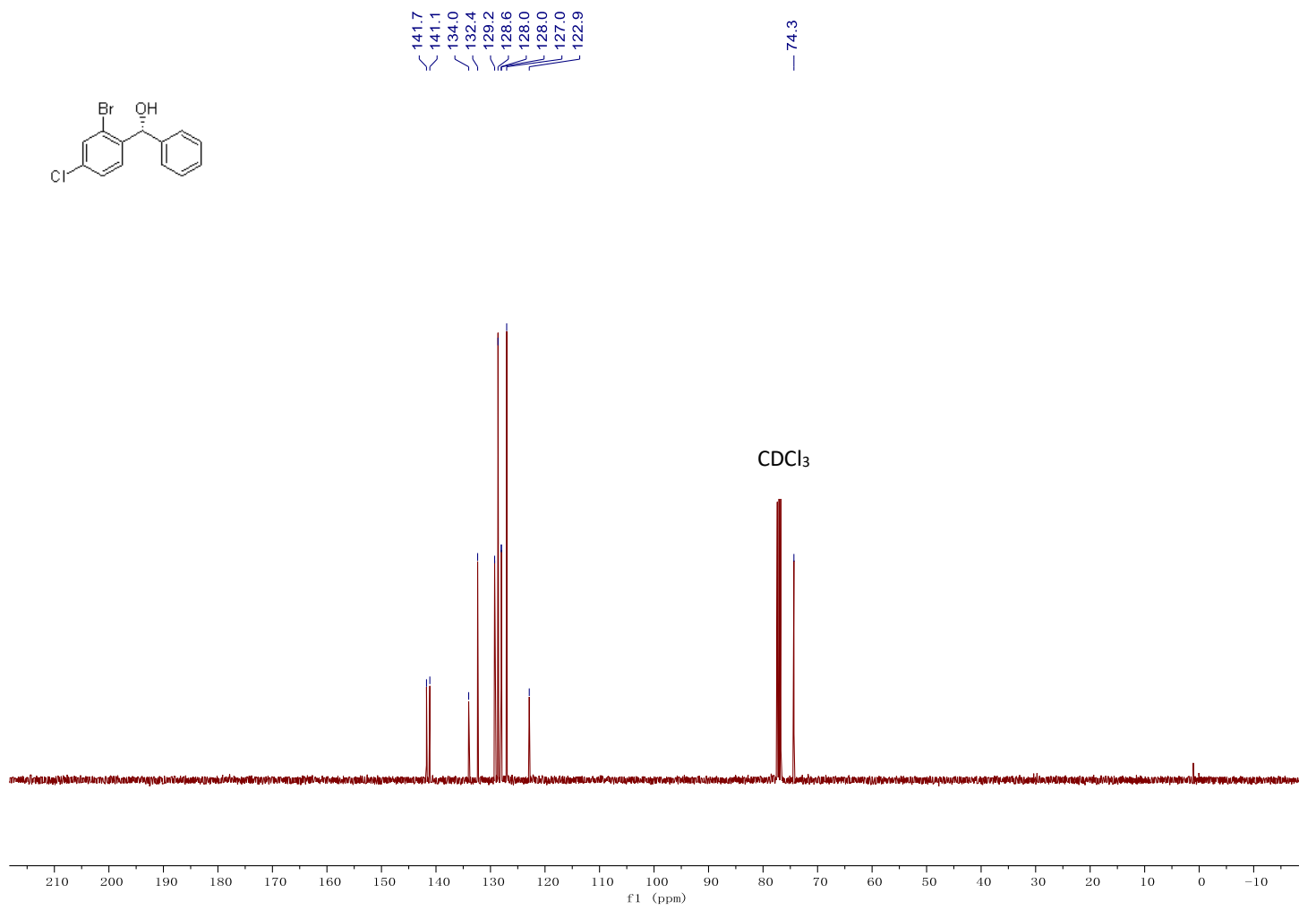
${ }^{1} \mathrm{H}$ NMR (600 MHz, $\left.\mathrm{CDCl}_{3}\right)$ of $10 \mathrm{t}$

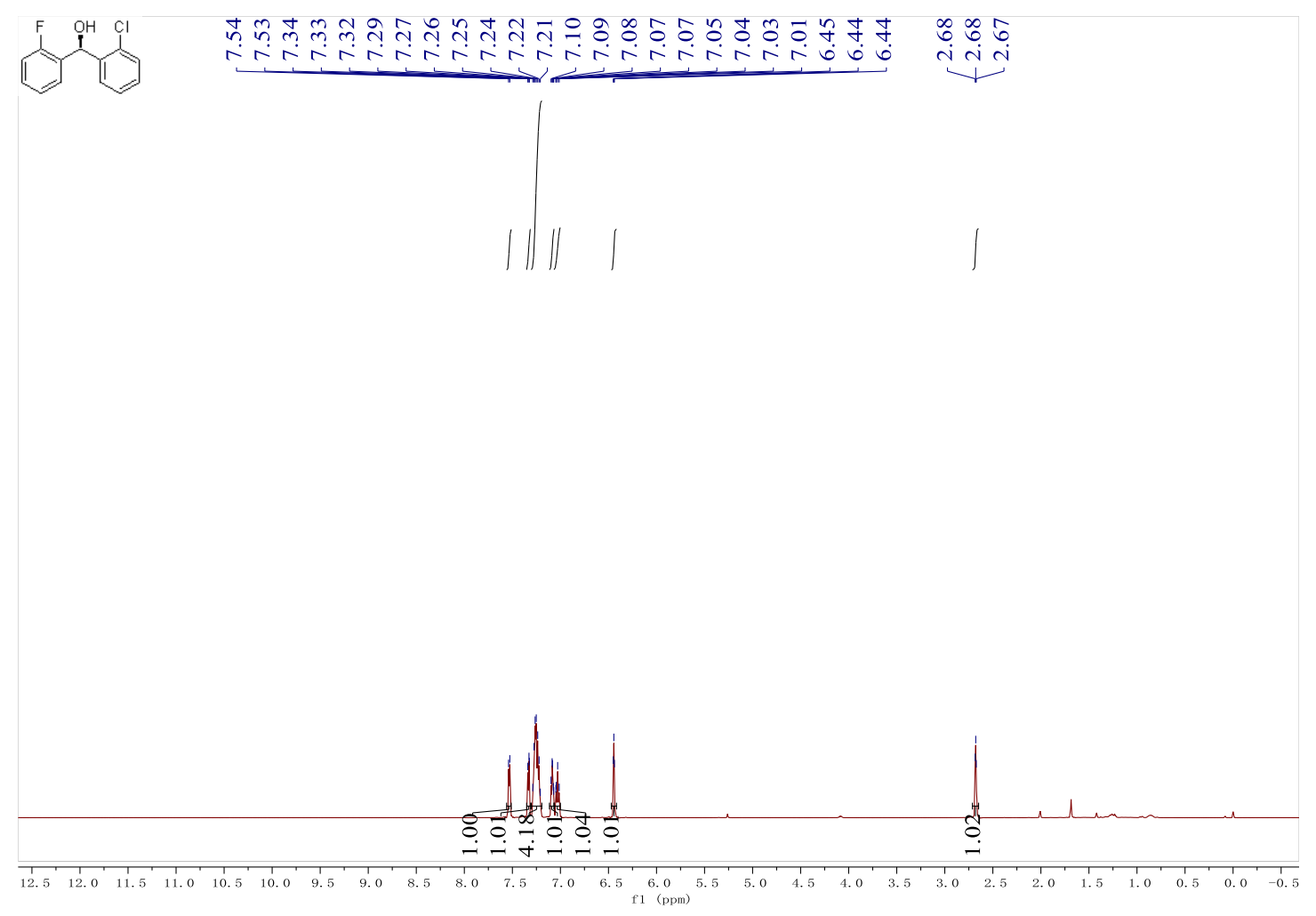

${ }^{13} \mathrm{C}$ NMR (101 MHz, $\left.\mathrm{CDCl}_{3}\right)$ of $10 \mathrm{t}$
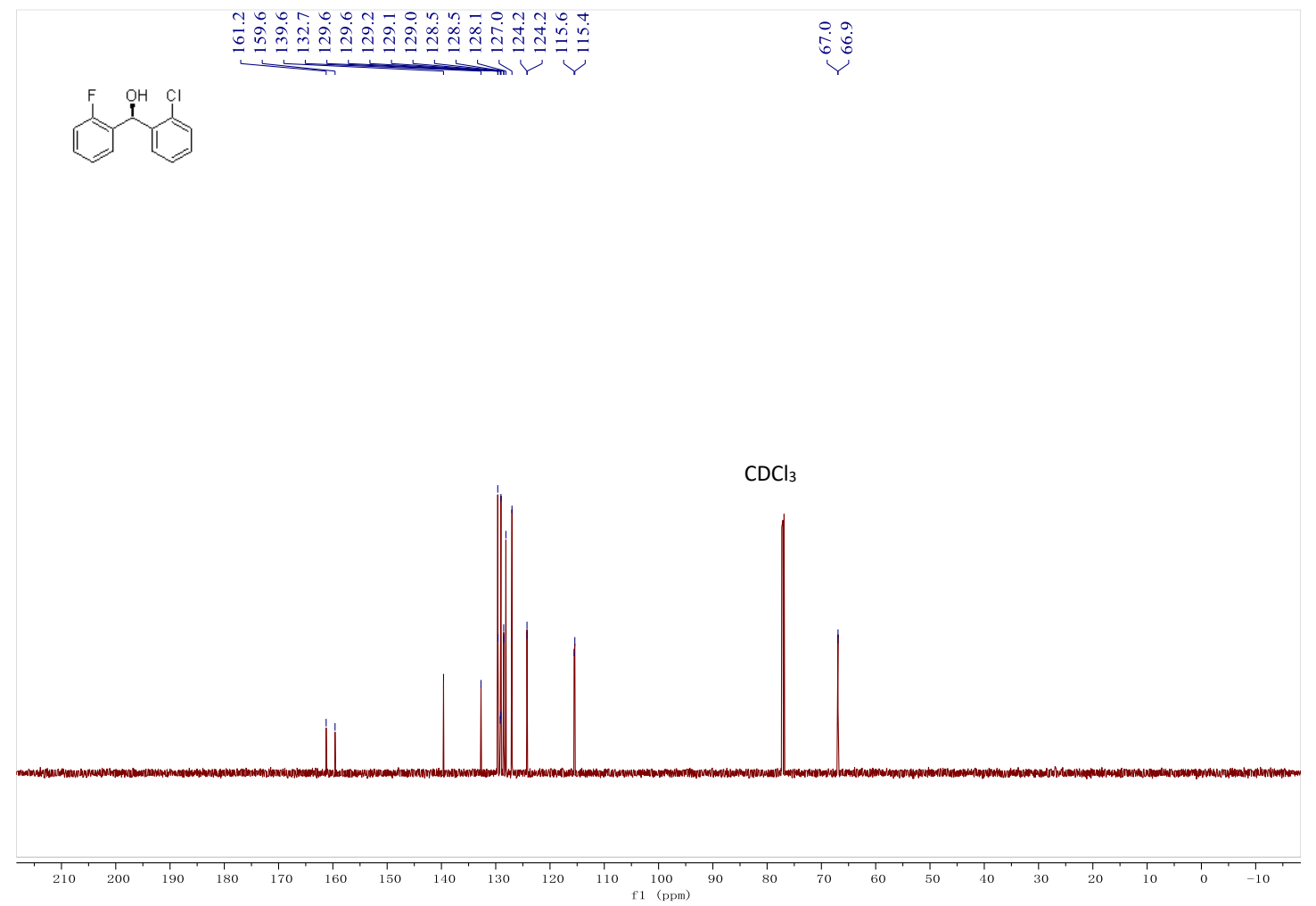
${ }^{19} \mathrm{~F}$ NMR (376 MHz, $\left.\mathrm{CDCl}_{3}\right)$ of $10 \mathrm{t}$

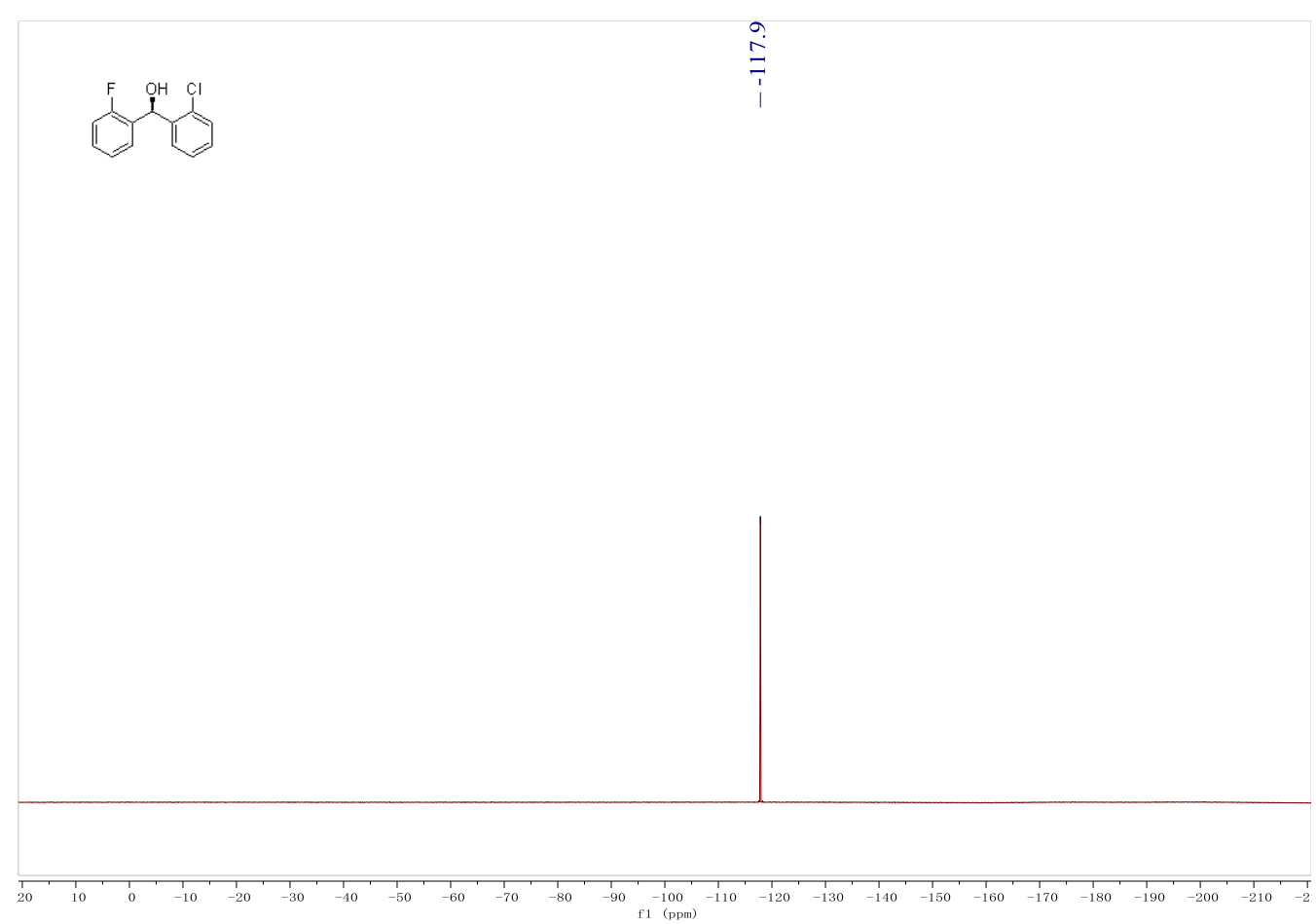


${ }^{1} \mathrm{H}$ NMR (400 MHz, $\left.\mathrm{CDCl}_{3}\right)$ of $10 \mathrm{u}$

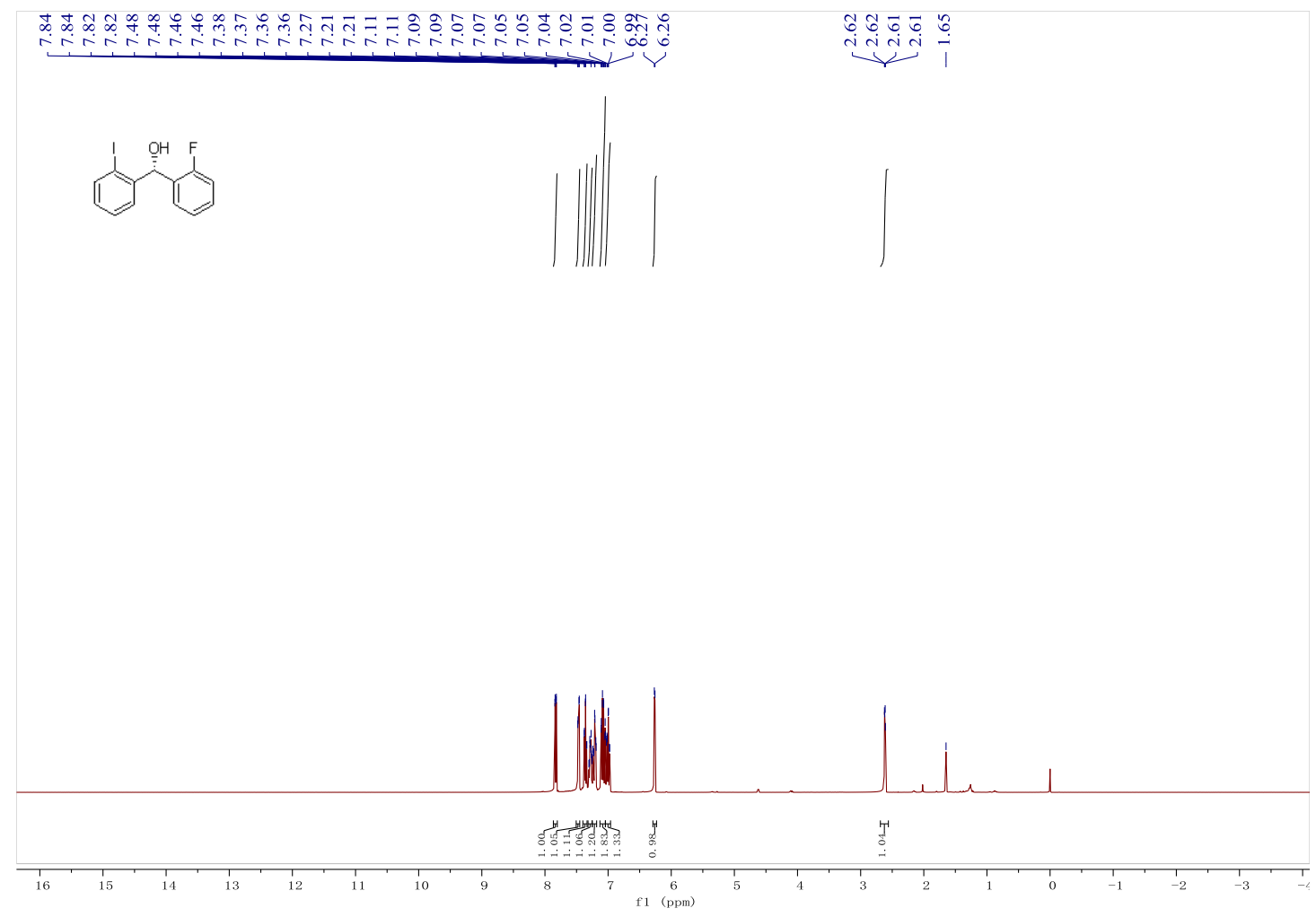

${ }^{13} \mathrm{C}$ NMR (151 MHz, CDCl 3$)$ of 10u
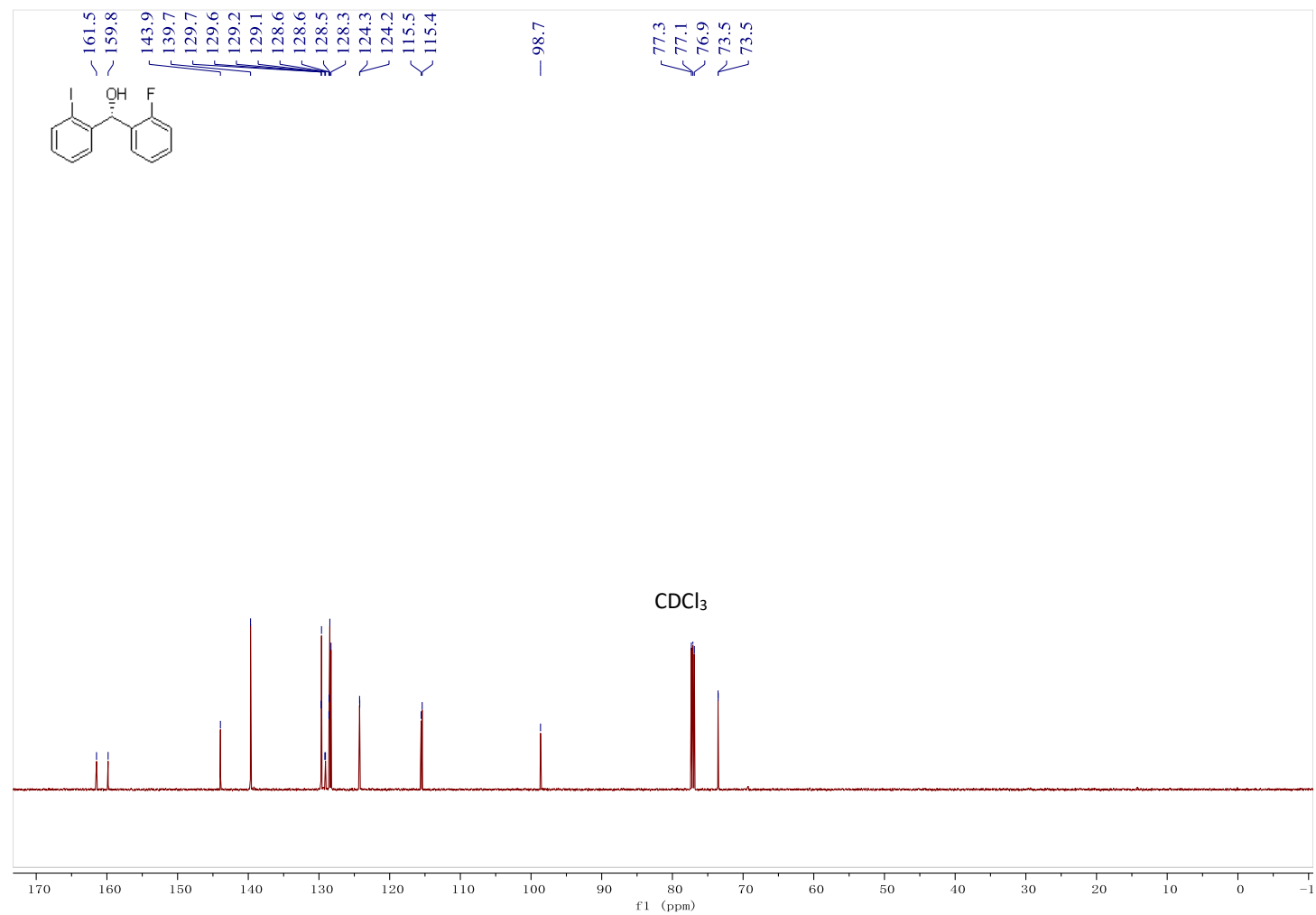
${ }^{19}$ F NMR (376 MHz, $\left.\mathrm{CDCl}_{3}\right)$ of $10 \mathrm{u}$

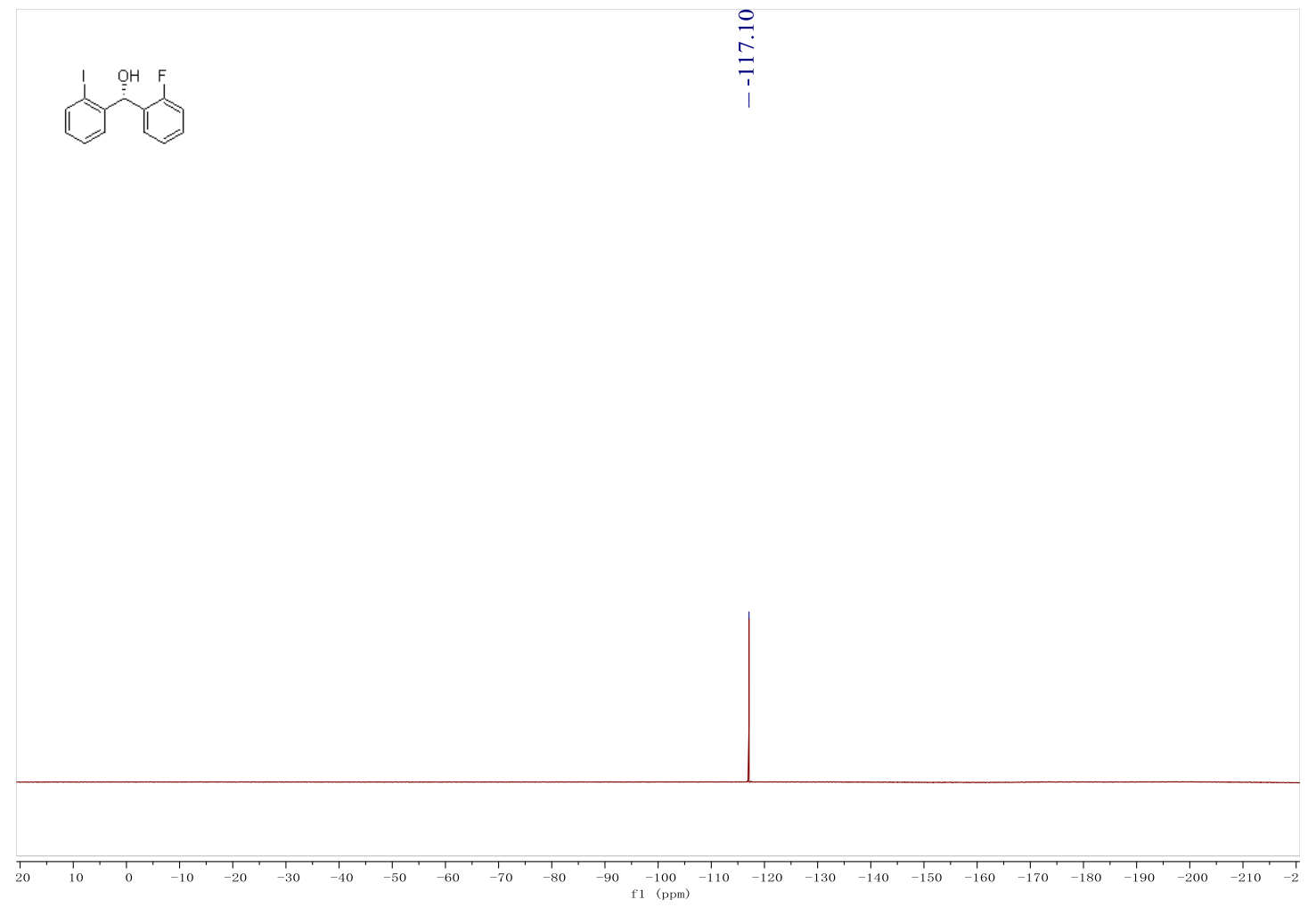


${ }^{1} \mathrm{H}$ NMR (600 MHz, $\left.\mathrm{CDCl}_{3}\right)$ of $10 \mathrm{v}$

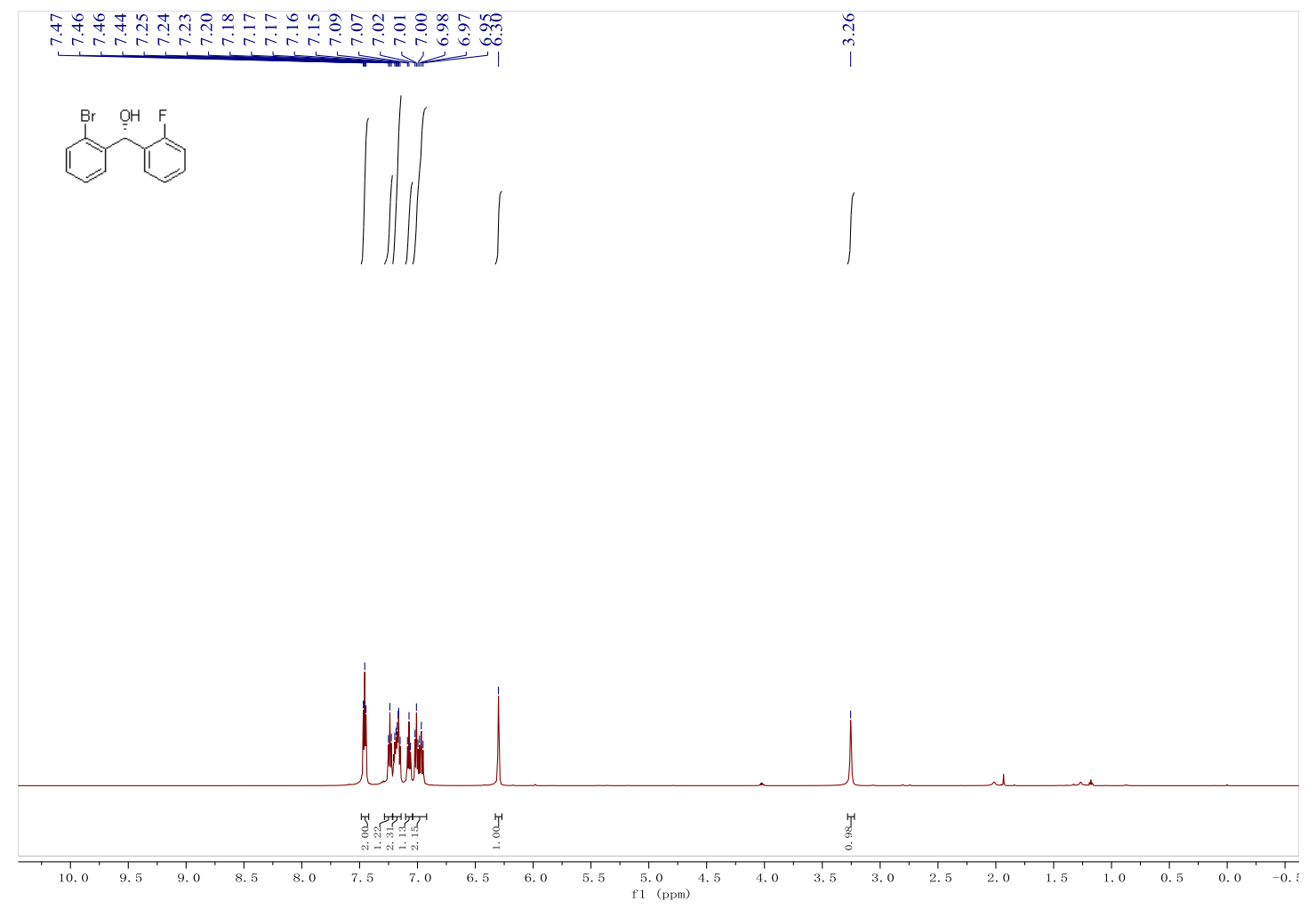

${ }^{13} \mathrm{C}$ NMR (151 MHz, CDCl 3$)$ of $10 \mathrm{v}$
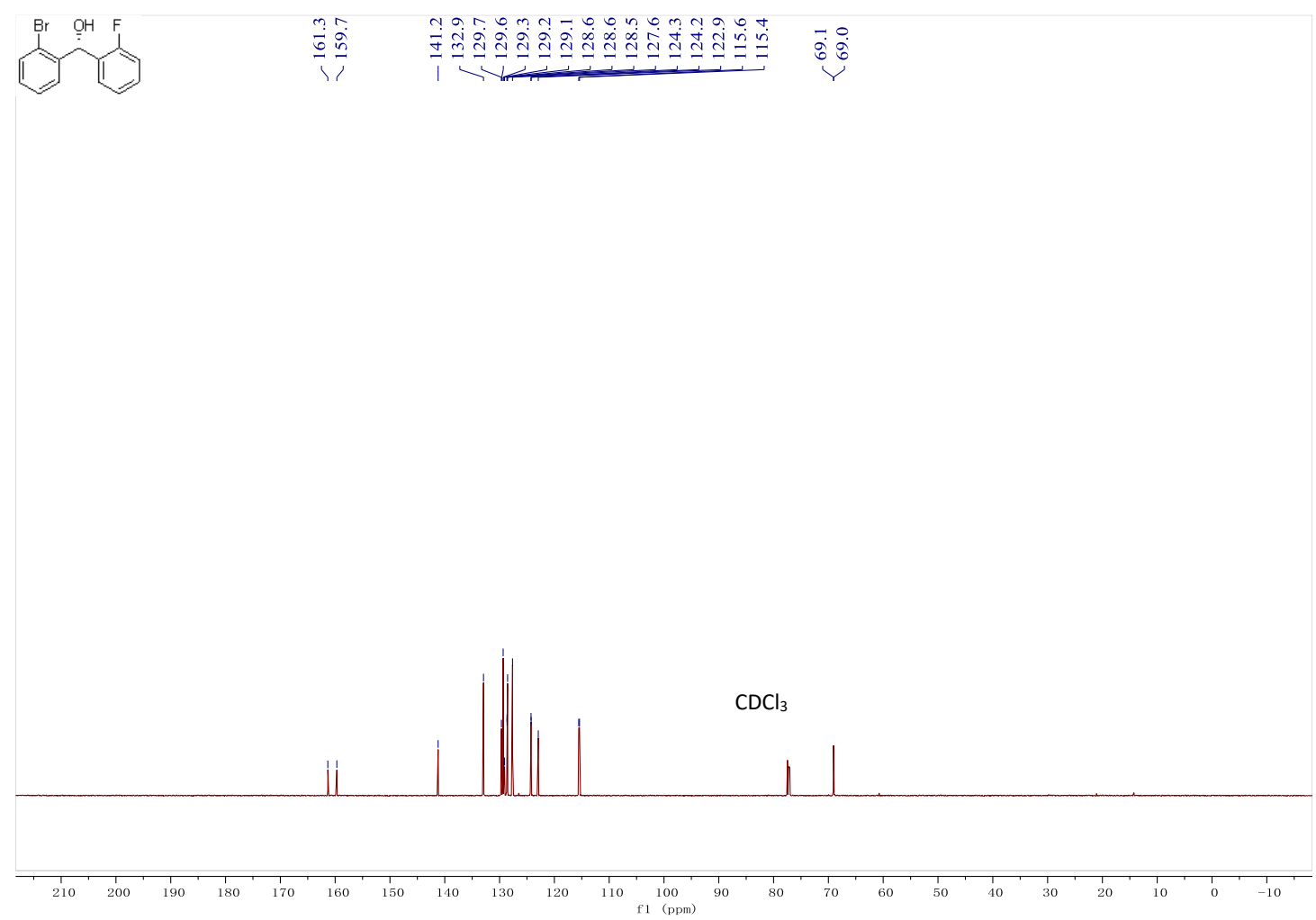
${ }^{19} \mathrm{~F}$ NMR (376 MHz, $\left.\mathrm{CDCl}_{3}\right)$ of 10v

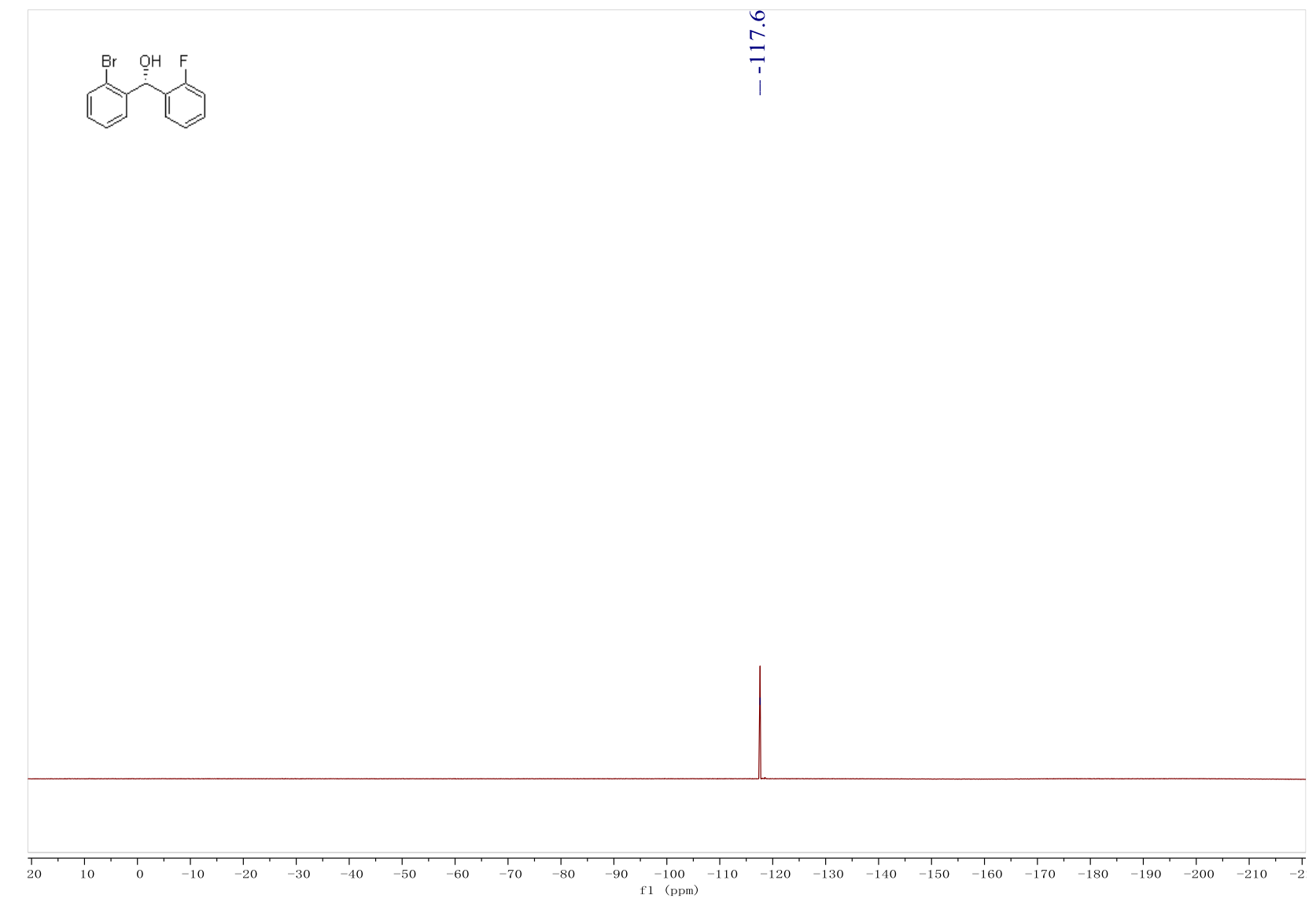


${ }^{1} \mathrm{H}$ NMR (400 MHz, CDCl 3$)$ of $10 \mathrm{w}$

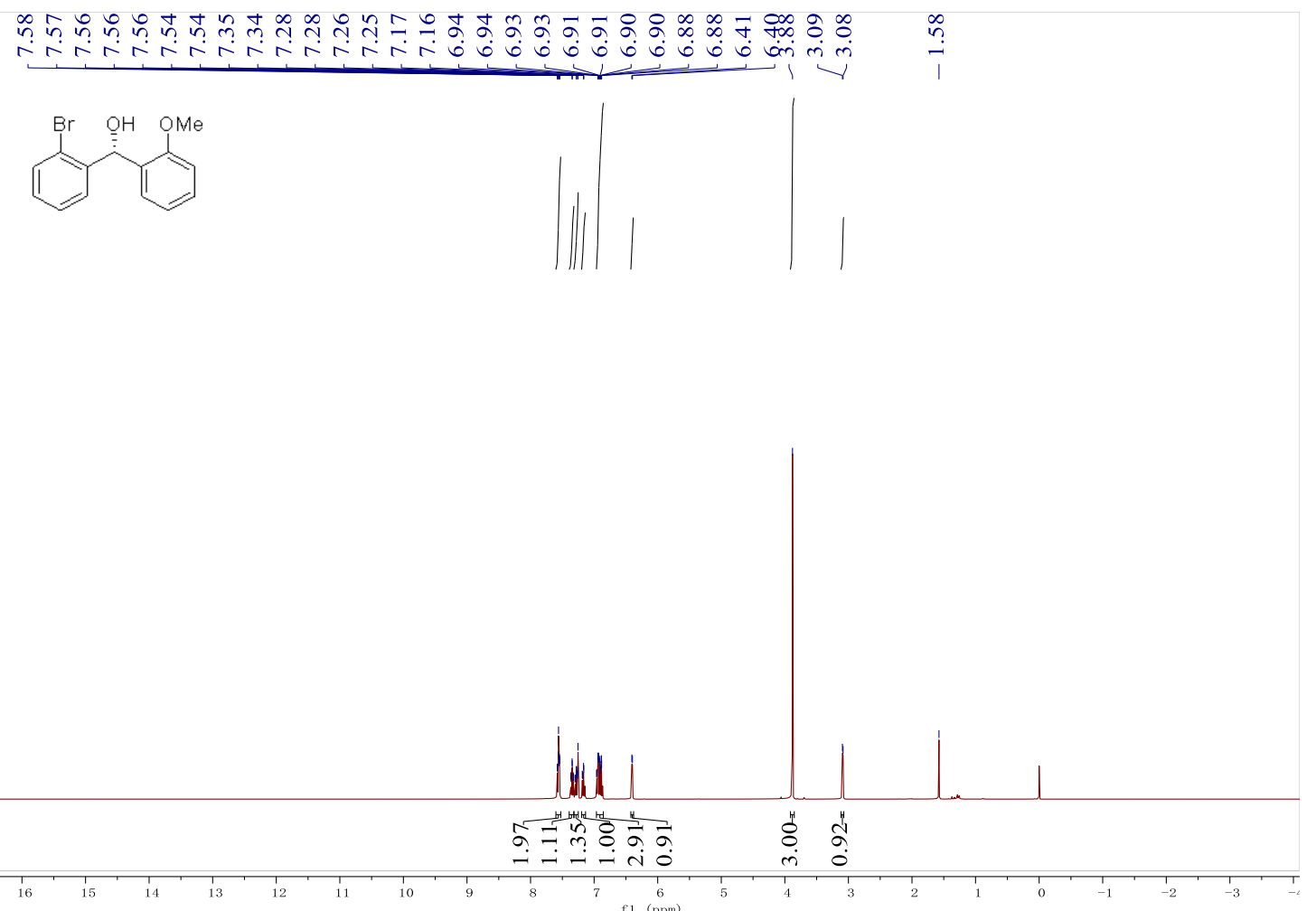

${ }^{13} \mathrm{C}$ NMR (101 MHz, $\left.\mathrm{CDCl}_{3}\right)$ of 10w
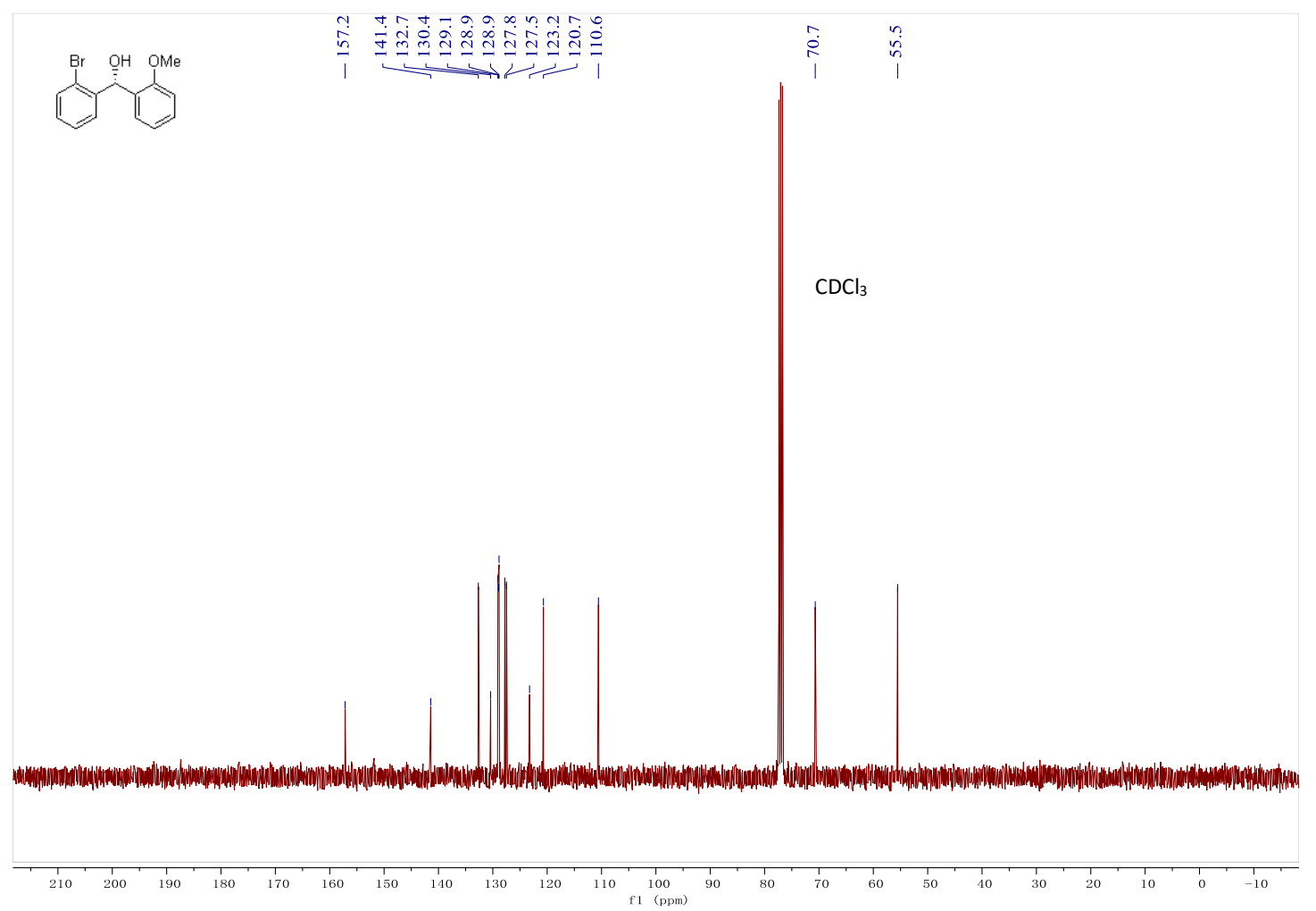


\section{References}

1. (a) Longeau, A.; Durand, S.; Spiegel, A.; Knochel, P. Sythesis of New C2-symmetrical diphosphines using chiral zinc organo-metallics. Tetrahedron Asymmetry. 1997, 8, 987-990. (b) Fujimura, O.; Grubbs, R. H. Asymmetric RingClosing Metathesis Catalyzed by Chiral Molybdenum Alkylidene Complex. The Journal of Organic Chemistry. 1998, 63, 824-832.

2. Zhang, X.; Huang, K.; Hou, G.; Cao, B.; Zhang, X. Angew. Chem., Int. Ed. 2010, 49, 6421.

3. Zhang, X-W.; Huang, K-X.; Hou, G-H.; Cao, B-N.; Zhang, X-M. Electron-Donating and Rigid P-Stereogenic Bisphospholane Ligands for Highly Enantioselective Rhodium-Catalyzed Asymmetric Hydrogenations. Angewandte Chemie International Edition. 2010, 49, 6421 -6424.

4. Miriyala, B.; Bhattacharyya, S.; Williamson, J. S. Chemoselec-tive reductive alkylation of ammonia with carbonyl com-pounds: synthesis of primary and symmetrical secondary amines. Tetrahedron. 2004, 60, 1463-1471.

5. Herde, J. L.; Lambert, J. C.; Senoff, C. V., Inorganic Syntheses. 1974, 15, 18-20.

6 (a) J. Meiners.; A. Friedrich.; E. Herdtweck.; S. Schneider. Organometallics. 2009, 28, 6331-6338. (b) T. W. Graham.; C.-W. Tsang.; X. Chen.; R. Guo.; W. Jia.; S.-M. Lu.; C. Sui-Seng.; C. B. Ewart.; A. Lough.; D. Amoroso.; K. Abdur-Rashid. Angew. Chem. Int. Ed. 2010, 49, $8708-8711$.

7. Chebolu, R.; Bahuguna, A.; Sharma, R.; Mishra, V. K.; Ravikumar, P. C., An unusual chemoselective oxidation strategy by an unprecedented exploration of an electrophilic center of DMSO: a new facet to classical DMSO oxidation. Chemical Communications, 2015, 51, 15438-15441.

8. Lei, P.; Meng, G.; Ling, Y.; An, J.; Nolan, S. P.; Szostak, M., General Method for the Suzuki-Miyaura CrossCoupling of Primary Amide-Derived Electrophiles Enabled by $[\mathrm{Pd}(\mathrm{NHC})(\mathrm{cin}) \mathrm{Cl}]$ at Room Temperature. Organic Letters, 2017, 19, 6510-6513.

9. Sloan, N. L.; Luthra, S. K.; McRobbie, G.; Pimlott, S. L.; Sutherland, A., A one-pot radioiodination of aryl amines via stable diazonium salts: preparation of 125I-imaging agents. Chemical Communications 2017, 53, $11008-11011$.

10. Kobayashi, E.; Kishi, A.; Togo, H., 6-Arylphenanthridines from Aryl o-Biaryl Ketones with 1,1,1,3,3,3Hexamethyldisilazane and Molecular Iodine. European Journal of Organic Chemistry 2019, 44, 7335-7347.

11. Ismael, A.; Skrydstrup, T.; Bayer, A., Carbonylative Suzuki-Miyaura couplings of sterically hindered aryl halides: synthesis of 2-aroylbenzoate derivatives. Organic \& Biomolecular Chemistry 2020, 18, 1754-1759.

12. Kobayashi, K.; Nakagawa, K.; Yuba, S.; Komatsu, T., Synthesis of 10-Aryl- and 10-(Arylmethyl)acridin-9(10H)ones via the Reaction of (2-Fluorophenyl)(2-halophenyl)methanones with Benzenamines and Arylmethanamines. Helvetica Chimica Acta 2013, 96, 389-396.

13. Gabbutt, C. D.; Heron, B. M.; Instone, A. C., The synthesis and electronic absorption spectra of 3-phenyl-3(4pyrrolidino-2-substituted phenyl)-3H-naphtho[2,1-b]pyrans: further exploration of the ortho substituent effect. Tetrahedron 2006, 62, 737-745.

14. Heinz, B.; Djukanovic, D.; Ganiek, M. A.; Martin, B.; Schenkel, B.; Knochel, P., Selective Acylation of Aryl- and Heteroarylmagnesium Reagents with Esters in Continuous Flow. Organic Letters 2020, 22, 493-496.

15. Niakan, M.; Asadi, Z.; Emami, M., Binuclear Palladium Complex Immobilized on Mesoporous SBA-16: Efficient Heterogeneous Catalyst for the Carbonylative Suzuki Coupling Reaction of Aryl Iodides and Arylboronic Acids Using Cr(CO)6 as Carbonyl Source. Catalysis Letters 2020, 150, 404-418.

16. Lei, C.; Zhu, D.; Tangcueco, V. I. I. I. T.; Zhou, J. S., Arylation of Aldehydes To Directly Form Ketones via Tandem Nickel Catalysis. Organic Letters 2019, 21, 5817-5822.

17. Gaikwad, V. V.; Mane, P. A.; Dey, S.; Bhanage, B. M., Xantphos-ligated palladium dithiolates: An unprecedented and convenient catalyst for the carbonylative Suzuki-Miyaura cross-coupling reaction with high turnover number and turnover frequency. Applied Organometallic Chemistry 2020, 34, e5255.

18. Jarugu Narasimha, M.; Subhas, S.; Apurba, L. K.; Werner, M. N., Steady-state photochemistry (Pschorr cyclization) and nanosecond transient absorption spectroscopy of twisted 2-bromoaryl ketones. Pure and Applied Chemistry 2011, $83,841-860$.

19. Zhang, L.; Tang, Y.; Han, Z.; Ding, K., Lutidine-Based Chiral Pincer Manganese Catalysts for Enantioselective Hydrogenation of Ketones. Angewandte Chemie International Edition 2019, 58, 4973-4977.

20. Bhattacharjee, S.; Guin, A.; Gaykar, R. N.; Biju, A. T., Iodide as a Nucleophilic Trigger in Aryne Three-Component Coupling for the Synthesis of 2-Iodobenzyl Alcohols. Organic Letters 2019, 21, 4383-4387.

21. He, W.-P.; Zhou, B.-H.; Zhou, Y.-L.; Li, X.-R.; Fan, L.-M.; Shou, H.-W.; Li, J., Synthesis of new benzimidazolium salts and their application in the asymmetric arylation of aldehydes. Tetrahedron Letters 2016, 57, 3152-3155.

22. Peng, Z.; Li, N.; Sun, X.; Wang, F.; Xu, L.; Jiang, C.; Song, L.; Yan, Z.-F., The transition-metal-catalyst-free oxidative homocoupling of organomanganese reagents prepared by the insertion of magnesium into organic halides in the presence of $\mathrm{MnCl} 2 \cdot 2 \mathrm{LiCl}$. Organic \& Biomolecular Chemistry 2014, 12, 7800-7809. 
23. Liu, T.-P.; Liao, Y.-X.; Xing, C.-H.; Hu, Q.-S., Fluorenone Synthesis by Palladacycle-Catalyzed Sequential Reactions of 2-Bromobenzaldehydes with Arylboronic Acids. Organic Letters 2011, 13, 2452-2455.

24. Furuta, K.; Kohno, S.; Shirahata, T.; Misaki, Y., Molecular Conductors with Effectively Half-Filled Electronic States Based on Tetrathiafulvalene Derivatives Condensed with a 2-Isopropylidene-1,3-dithiole Ring. European Journal of Inorganic Chemistry 2014, 24, 3982-3988.

25. Mohapatra, H.; Phillips, S. T., Using Smell To Triage Samples in Point-of-Care Assays. Angewandte Chemie International Edition 2012, 51, 11145-11148.

26. Sui, Y.-Z.; Zhang, X.-C.; Wu, J.-W.; Li, S.; Zhou, J.-N.; Li, M.; Fang, W.; Chan, A. S. C.; Wu, J., CuII-Catalyzed Asymmetric Hydrosilylation of Diaryl- and Aryl Heteroaryl Ketones: Application in the Enantioselective Synthesis of Orphenadrine and Neobenodine. Chemistry - A European Journal 2012, 18, 7486-7492.

27. Arao, T.; Kondo, K.; Aoyama, T., Nickel-catalyzed 1,2-addition of arylboroxines to aromatic aldehydes. Tetrahedron Letters 2007, 48, 4115-4117.

28. Ling, F.; Nian, S.; Chen, J.; Luo, W.; Wang, Z.; Lv, Y.; Zhong, W., Development of Ferrocene-Based DiaminePhosphine-Sulfonamide Ligands for Iridium-Catalyzed Asymmetric Hydrogenation of Ketones. The Journal of Organic Chemistry 2018, 83, 10749-10761.

29. Lockner, J. W.; Dixon, D. D.; Risgaard, R.; Baran, P. S., Practical Radical Cyclizations with Arylboronic Acids and Trifluoroborates. Organic Letters 2011, 13, 5628-5631.

30. Zhuo, S.; Zhu, T.; Zhou, L.; Mou, C.; Chai, H.; Lu, Y.; Pan, L.; Jin, Z.; Chi, Y. R., Access to All-Carbon Spirocycles through a Carbene and Thiourea Cocatalytic Desymmetrization Cascade Reaction. Angewandte Chemie International Edition 2019, 58, 1784-1788.

31. Karthikeyan, J.; Jeganmohan, M.; Cheng, C.-H., Cobalt-Catalyzed Addition Reaction of Organoboronic Acids with Aldehydes: Highly Enantioselective Synthesis of Diarylmethanols. Chemistry - A European Journal, 2010, 16, 8989-8992.

32. Hartman, G. D.; Halczenko, W.; Phillips, B. T., Iminium ion mediated cyclizations with 4-aryl-1,4dihydropyridines. Bridging with thiophene and furan. The Journal of Organic Chemistry 1986, 51, 142-148.

33. Lange, S.; Elangovan, S.; Cordes, C.; Spannenberg, A.; Jiao, H-J.; Junge, H.; Bachmann, S.; Scalone, M.; Topf, C.; Junge, K.; Beller, M. Catal. Sci. Technol., 2016, 6, 4768-4772.

34. Tomasi, J.; Mennucci, B.; Cammi, R. Quantum Mechanical Continuum Solvation Models. Chemical Reviews, 2005, 105, 2999-3094.

35. Frisch, M. J.; Trucks, G. W.; Schlegel, H. B.; Scuseria, G. E.; Robb, M. A.; Cheeseman, J. R.; Scalmani, G.; Barone, V.; Mennucci, B.; Petersson, G. A.; Nakatsuji, H.; Caricato, M.; Li, X.; Hratchian, H. P.; Izmaylov, A. F.; Bloino, J.; Zheng, G.; Sonnenberg, J. L.; Hada, M. E., M.; ; Toyota, K. F., R.; ; Hasegawa, J.; Ishida, M.; Nakajima, T.; Honda, Y.; Kitao, O.; Nakai, H.; Vreven, T.; Montgomery, J., J. A.;; Peralta, J. E.; Ogliaro, F.; Bearpark, M.; Heyd, J. J.; Brothers, E.; Kudin, K. N.; Staroverov, V. N.; Kobayashi, R.; Normand, J.; Raghavachari, K.; Rendell, A.; Burant, J. C.; Iyengar, S. S.; Tomasi, J.; Cossi, M.; Rega, N.; Millam, N. J.; Klene, M.; Knox, J. E.; Cross, J. B.; Bakken, V.; Adamo, C.; Jaramillo, J.; Gomperts, R.; Stratmann, R. E.; Yazyev, O.; Austin, A. J.; Cammi, R.; Pomelli, C.; Ochterski, J. W.; Martin, R. L.; Morokuma, K.; Zakrzewski, V. G.; Voth, G. A.; Salvador, P.; Dannenberg, J. J.; Dapprich, S.; Daniels, A. D.; Farkas, Ö.; Foresman, J. B.; Ortiz, J. V.; Cioslowski, J.; Fox, D. J. Gaussian 09 D.01, Gaussian, Inc., Wallingford CT, 2013.

36. Chai, J.-D.; Head-Gordon, M. Long-range corrected hybrid density functionals with damped atom-atom dispersion corrections. Physical Chemistry Chemical Physics, 2008, 10, 6615-6620.

37. (a) Petersson, G. A.; Bennett, A.; Tensfeldt, T. G.; Al-Laham, M. A.; Shirley, W. A.; Mantzaris, J. A complete basis set model chemistry. I. The total energies of closed-shell atoms and hydrides of the first-row elements. The Journal of Chemical Physics, 1988, 89, 2193-2218. (b) Petersson, G. A.; Al-Laham, M. A. A complete basis set model chemistry. II. Open-shell systems and the total energies of the first-row atoms. The Journal of Chemical Physics, 1991, 94, 6081-6090. (c) Clark, T.; Chandrasekhar, J.; Spitznagel, G. W.; Schleyer, P. V. R. Efficient diffuse functionaugmented basis sets for anion calculations. III. The 3-21+G basis set for first-row elements, Li-F. 1983, 4, 294-301. 38. Ehlers, A.; Böhme, M.; Dapprich, S.; Gobbi, A.; Höllwarth, A.; Jonas, V.; Köhler, K.; Stegmann, R.; Veldkamp, A.; Frenking, G. A set of f-polarization functions for pseudo-potential basis sets of the transition metals $\mathrm{Sc}-\mathrm{Cu}, \mathrm{Y}-\mathrm{Ag}$ and La-Au. Chem. Phys. Lett. 1993, 208, 111-114.

39. Fukui, K. The path of chemical reactions-the IRC approach. Acc. Chem. Res. 1981, 14, 363-368. 